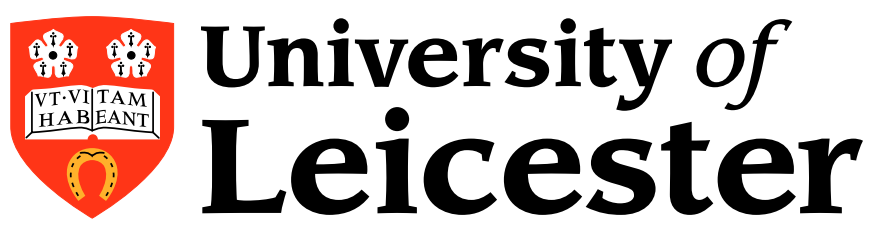

\title{
Ultracool Companions to White Dwarfs
}

\section{Paul Raymond Steele}

\author{
Supervisor: \\ Matthew Burleigh \\ A thesis submitted for the degree of \\ Doctor of Philosophy \\ at the University of Leicester \\ X-ray \& Observational Astronomy Group \\ Department of Physics \& Astronomy \\ University of Leicester
}

January 2010 


\section{Declaration}

I hereby declare that no part of this thesis has been previously submitted to this or any other University as part of the requirement for a higher degree. The work described herein was conducted by the undersigned, except for contributions from colleagues as acknowledged in the text.

Paul Raymond Steele

January 2010 


\title{
Ultracool Companions to White Dwarfs
}

\section{Paul Raymond Steele}

\begin{abstract}
In this thesis I present a new near-infrared photometric search for unresolved ultracool companions and debris disks to white dwarfs in UKIDSS DR5. 24 DA white dwarfs were found with multiple excesses indicative of a low mass companion, with 7 of these having a predicted mass in the range associated with brown dwarfs. The results of this survey show that the unresolved brown dwarf companion fraction to DA white dwarfs is $0.5 \leq f_{\mathrm{WD}+\mathrm{BD}} \leq 1.8 \pm 0.7 \%$. I also calculate the unresolved L-dwarf companion fraction to be $0.5 \leq f_{\mathrm{WD}+\mathrm{dL}} \leq 1.5 \pm 0.6 \%$, which is much higher than the previous estimate of Farihi, Becklin \& Zuckerman (2005). However, the results are consistent with previous estimates of the brown dwarf companion fraction to main sequence stars.

I analyse the near-infrared spectra of eleven known DA white dwarf + M-dwarf binaries, and compare the spectral types assigned using optical photometry against those assigned using near-infrared spectra. I search for evidence that the known short period systems once existed in a common envelope phase. No such evidence was found.

I also present the spectroscopic analysis of two particularly interesting binaries; PG 1234+ 482 and PHL 5038. PG 1234+482 is the hottest and youngest DA white dwarf with a cool companion on the stellar-substellar borderline. I discuss the possibility that the companion is a brown dwarf, and the possibility that the secondary is the source of contamination by heavy metals in the white dwarf's atmosphere. PHL 5038 was identified to have a near-infrared excess in UKIDSS, and the spectroscopic analysis in this thesis confirms that the secondary in this system can be resolved at a projected orbital separation of $55 \mathrm{AU}$, and is a brown dwarf with spectral type L8-L9. This is only the second such pair found (over 20 years later) after GD 165AB. The secondary in this system has the potential to be used as a benchmark brown dwarf for testing substellar atmospheric models.
\end{abstract}




\section{Publications}

A significant amount of work contained in this thesis has been published in the following papers:

Steele, P. R., Burleigh, M. R., Dobbie, P. D. and Barstow, M. A., "Near-Infrared spectroscopy of the very low mass companion to the hot DA white dwarf PG 1234+482", MNRAS, 2007, 382, 1804-1808

Steele, P. R., Burleigh, M. R., Barstow, M. A., Jameson, R. F. and Dobbie, P. D., "White dwarfs with unresolved substellar companions and debris disks in the UKIDSS Survey", '16th European Workshop on White Dwarfs', Vol. 172, Journal of Physics Conference Series

Steele, P. R., Burleigh, M. R., Farihi, J., Gänsicke, B. T., Jameson, R. F., Dobbie, P. D. and Barstow, M. A., "PHL 5038: A spatially resolved white dwarf + brown dwarf binary", A\&A, 2009, 500, 1207-1210 


\section{Acknowledgements}

My first introduction into the field of white dwarfs came in the $3^{\text {rd }}$ year of my undergraduate degree at Leicester. On the project list was the promise of 'Extrasolar planets', under the guidance of my future supervisor Matt Burleigh. Unfortunately, it was to also be my first experience of real time astronomy and weather not mixing. However, this lead me onto a project involving transiting objects around white dwarfs, launching me into an area which was relatively unknown to me at the time. A few years later the opportunity arose to once again study the subject, and so here I am now at the end of my $\mathrm{PhD}$ ! So first and foremost I would like to thank Matt for his guidance over the years, and also for his knowledge of Britain's (and other countries) best pubs. Cheers Matt.

I would also like to thank all my colleagues and fellow $\mathrm{PhD}$ students at the University of Leicester. In particular Chris, who survived the undergraduate years with me, and suffered through the 'black madness' at the RAS NAM 2009. I also thank my fellow office buddys from across the few years I have spent at Leicester. Small Dave, Big Dave (Who can use his eyebrows as astronomical baffles), Emma and Nathan. You may now fight over who gets my desk.

Last, but by no means least I would like to thank my wife Mary, who has carried on with her career whilst I still swam the murky depths of student life. If it wasn't for my $10 \%$ discount at New Look I don't know if she would have carried on putting up with me. Thank you for believing in me. 
For John Bryan

1953-2009 


\section{Contents}

1 Introduction 1

1.1 White Dwarfs .......................... 1

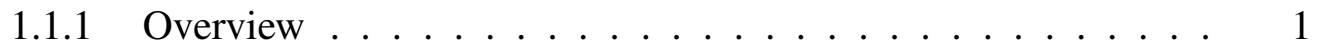

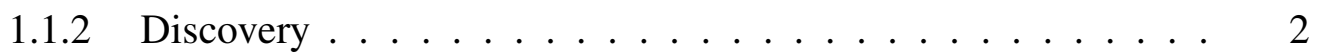

1.1.3 White Dwarf Structure . . . . . . . . . . . . 4

1.1.4 White Dwarf Classification . . . . . . . . . . 8

1.1.5 White Dwarf Formation ............... 11

1.1.6 White Dwarf Evolution . . . . . . . . . . . . 18

1.2 White Dwarfs in Binaries . . . . . . . . . . . . 20

1.2.1 Common Envelope Evolution . . . . . . . . . . . 25

1.2.2 Brown Dwarfs . . . . . . . . . . . . . . 28

1.2.3 Brown Dwarf Atmospheres ............. . 29

1.2.4 White Dwarfs with Sub-Stellar Companions . . . . . . . . . . 30

1.3 White Dwarfs with Debris Disks ............... 31 
2 White Dwarfs in UKIDSS

2.1 Overview ............................ 34

2.2 The UKIDSS Survey $\ldots \ldots \ldots \ldots \ldots$

2.2.1 The Large Area Survey . . . . . . . . . . . . . . . . . 35

2.3 Candidate Selection . . . . . . . . . . . . . . . . . 37

2.3.1 Identifying White Dwarfs $\ldots \ldots \ldots \ldots$. . . . . . 37

2.3.2 The Near-infrared Two Colour Diagram . . . . . . . . . . . 39

2.3.3 White Dwarf Modelling . . . . . . . . . . . . . 47

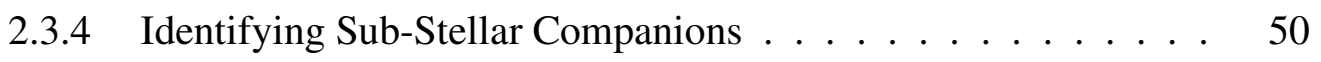

2.3.5 Identifying Debris Disks . . . . . . . . . . . . . . 55

2.4 Follow-up Near-infrared Photometric Observations $\ldots \ldots \ldots$

2.5 Candidate UKIDSS Photometry $\ldots \ldots \ldots \ldots$

2.5.1 Examples of White Dwarfs with a NIR Excess Indicative of a Low Mass Companion . . . . . . . . . . . . . . . . . . 59

2.5.2 Magnetic White Dwarfs . . . . . . . . . . . . . 74

2.5 .3 Debris Disks . . . . . . . . . . . . . . . . . 79

2.6 Low Mass Star or Brown Dwarf? . . . . . . . . . . . . . . . 82

2.7 A Wide or Close Binary? . . . . . . . . . . . . . . . . . . 87

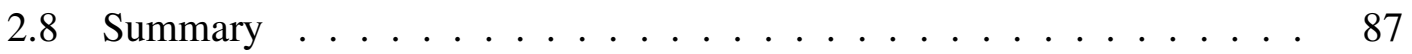


3 NIR Spectroscopic Observations of WD + dM Binaries 9

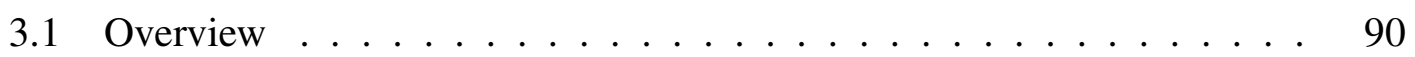

3.2 Observations $\ldots \ldots \ldots \ldots \ldots \ldots \ldots \ldots \ldots \ldots \ldots$

3.3 Data Reduction . . . . . . . . . . . . . . . . . . . . . . 94

3.4 Analysis of Data $\ldots \ldots \ldots \ldots \ldots$

3.4.1 White Dwarf Model Spectra . . . . . . . . . . . . . . . 97

3.4.2 Spectrally Typing Low Mass Stellar Companions . . . . . . . 97

3.5 Results . . . . . . . . . . . . . . . . . . . . . 101

3.5.1 Examples of the Spectroscopic Classification of a Companion . 101

3.5.2 Constraining Spectral Types Through Na I Line Strengths _ . . 106

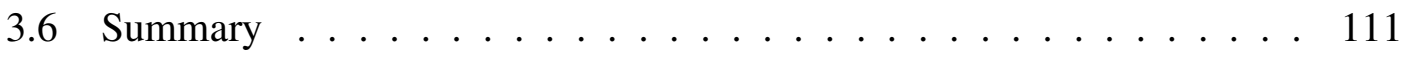

4 PG 1234+482: A Borderline Substellar Companion 113

4.1 Overview . . . . . . . . . . . . . . . 113

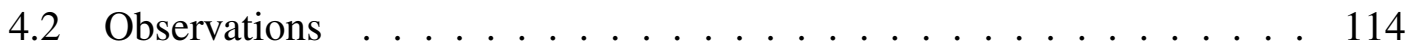

4.3 Data Reduction . . . . . . . . . . . . . . . . . . 115

4.4 Data Analysis . . . . . . . . . . . . . . . . . . . . . 116

4.5 Results . . . . . . . . . . . . . . . . . . . . 120

4.6 A Boundary Stellar-Substellar Companion . . . . . . . . . . . . . 121

4.7 Summary . . . . . . . . . . . . . . . . . . . . 122 


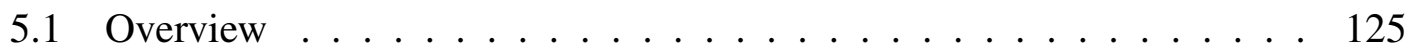

5.2 Observations . . . . . . . . . . . . . . . 127

5.3 Data Reduction . . . . . . . . . . . . . . . . . . . . . 128

5.3.1 Photometry . . . . . . . . . . . . . . . . . . . . . . . 129

$5.3 .2 \quad$ Spectroscopy . . . . . . . . . . . . . . . . 130

5.4 Data Analysis . . . . . . . . . . . . . . . . . . . 131

5.5 Discussion . . . . . . . . . . . . . . . . . . . 134

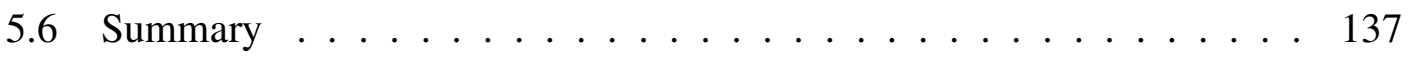

6 Discussion 139

6.1 Overview . . . . . . . . . . . . . . . . . 139

6.2 Limits on Unresolved Ultracool Companions to DA White Dwarfs . . 140

6.3 Survey Sensitivity . . . . . . . . . . . . . . . . . . . . 145

6.4 Binary Statistics for White Dwarfs with Unresolved Substellar Companions 148

6.5 Comparison to Previous Estimates $\ldots \ldots \ldots \ldots$. . . . . . 157

6.5.1 The White Dwarf + L-Dwarf Binary Fraction . . . . . . . 157

6.5.2 The Main-sequence Star + Brown Dwarf Binary Fraction _ . 164

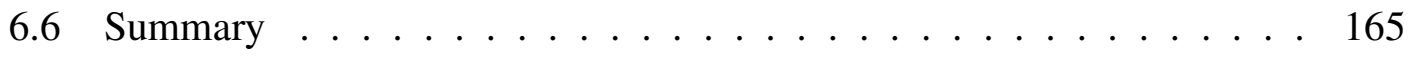


$7 \quad$ Summary and Future Work $\quad 167$

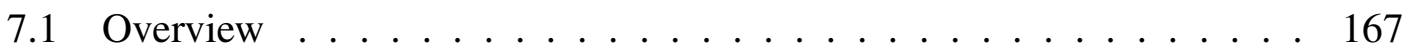

7.2 Summary of Results . . . . . . . . . . . . . . . . . . 167

7.3 Future Work . . . . . . . . . . . . . . . 171

7.3.1 Follow-up Observations . . . . . . . . . . . 171

7.3.2 The Future of UKIDSS . . . . . . . . . . . . . . . 172

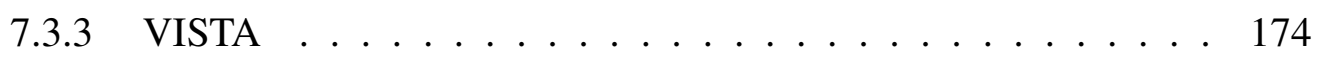

7.3 .4 WISE ......................... 177

$\begin{array}{lll}\text { A Chapter 2: Spectra } & 181\end{array}$

B Chapter 3: Individual Discussions and Spectra 192

C UKIDSS White Dwarf Data 206 


\section{List of Figures}

1.1 Hertzsprung-Russel Diagram showing the positions of 40 Eridani B and

Sirius B, now known to be white dwarfs. . . . . . . . . . . . . .

1.2 Age vs. effective temperature for both a $0.6 M_{\odot}$ and $1.0 M_{\odot}$ white dwarf showing how cooling slows with white dwarf age. Curves are based on the models of Wood (1994). . . . . . . . . . . . . .

1.3 The Hertzsprung-Russell diagram, showing the evolutionary paths of stars from the main sequence to the white dwarf cooling sequence (reproduced from Marsh 1995). . . . . . . . . . . . . .

1.4 Hertzsprung-Russel Diagram showing the positions of main sequence stars, white dwarfs, red giants and supergiants. . . . . . . . . .

1.5 Simulated binary separation distribution for white dwarfs assuming an initial separation distribution similar to that of G-dwarf binaries. Figure from Farihi (2004). . . . . . . . . . . . . . . . . 
1.6 (a) Predicted final outcome of CEE for different planetary masses and initial periods around a $1.0 M_{\odot}$ star. The solid line represents the critical mass below which the planet will evaporate during the spiral-in phase. The shaded region to the right shows where a planet would be too far away to be engulfed by an envelope whilst the star is on the RGB. (b) Final mass of the white dwarf in the case where all the envelope is expelled. Reproduced from Nelemans \& Tauris (1998) . . . . . . . . . . . .

2.1 Planned final LAS sky coverage. The equatorial block (EB) corresponds to SDSS stripes 9 to 16 , the northern block (NB) corresponds to SDSS stripes 26 to 33, and the southern stripe (SS) is part of SDSS stripe 82 . The dashed line marks the Galactic plane, and the dotted line marks the

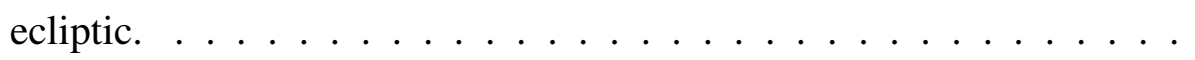

2.2 $J-H$ vs $H-K$ colour-colour diagram for the SDSS white dwarfs (top) and the McCook \& Sion white dwarfs (bottom) with complete JHK photometry present in the UKIDSS archive. $\mathrm{Nb}$. the overlap between the 2 catalogues has not been accounted for in these diagrams. The four regions delineate (I) single white dwarfs, (II) white dwarfs with latetype main-sequence companions, (III) white dwarfs with lower mass, possible brown dwarf companions and (IV) white dwarfs contaminated with circumstellar material. . . . . . . . . . . . . .

2.3 Near-infrared colour-colour diagram of white dwarfs with confirmed lowmass companions (purple) and circumstellar disks (red). . . . . . . 
2.4 SDSS finder image of SDSS J020538.12+005835.3 clearly showing both system components. . . . . . . . . . . . . . . . . . . 48 48

2.5 UKIDSS $Y J H K$ images of SDSS J020538.12+005835.3 $\ldots \ldots \ldots$

2.6 Absolute $K$-band photometry verses spectral type for L and T-dwarfs (Red: Reproduced from Vrba et al. 2004). Also plotted are the predicted absolute magnitudes of the candidate companions in this survey (blue).

2.7 SDSS J003902.47-003000.3 model spectrum (black solid) with SDSS griz' (blue), UKIDSS $Y J H K$ (red) and NTT $J H K$ photometry (purple). Also shown are the SDSS spectrum (light grey) and a composite WD+L0 spectrum (dark grey). . . . . . . . . . . .

2.8 SDSS J012032.27-001351.1 model spectrum (black solid) with SDSS griz' (blue) and UKIDSS $Y J H K$ photometry (red). Also shown is the SDSS spectrum (light grey) and an M7 spectrum scaled to the $K$-band magnitude of the putative secondary. . . . . . . . . . . .

2.9 SDSS finder image of SDSS J012032.27-001351.1. A red object can be seen just to the south of the blue white dwarf. . . . . . . . . . .

2.10 UKIDSS $Y J H K$ images of SDSS J012032.27-001351.1. Both objects from the SDSS image can be seen in the UKIDSS $Y$-band, with only the southern most object appearing in the $J H K$ images. . . . . . . . . 
2.11 SDSS J013532.98+144555.8 model spectrum (black solid) with SDSS griz' (blue) and UKIDSS $Y J H K$ photometry (red). Also shown are the SDSS spectrum (light grey) and a composite WD+L5 spectrum (dark

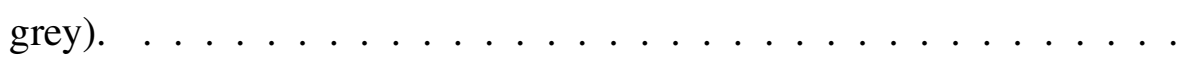

2.12 POSS I and POSS II images of NLTT 5306, with the current and prior positions of the star respectively marked. Also shown is the current epoch finder image from SDSS, and a composite $J H K$ image from the

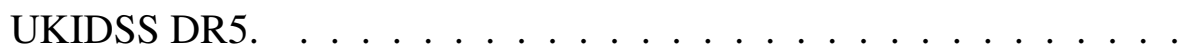

2.13 SDSS J023247.50-003909.3 model spectrum (black solid) with SDSS griz' (blue) and UKIDSS $J H K$ photometry (red). Also shown is the SDSS spectrum (light grey) and an L3 spectrum scaled to the $K$-band magnitude of the putative secondary. . . . . . . . . . . . .

2.14 SDSS finder image of SDSS J023247.50-003909.3. A red object can be seen just to the south west of the blue white dwarf. . . . . . . . . .

2.15 UKIDSS $J H K$ images of SDSS J023247.50-003909.3. The white dwarf has almost completely faded from view in the $J$-band image, with the red object completely dominating in the $H$ and $K$-bands. . . . . . . . . . 68

2.16 WD 0922+006 model spectrum (solid black) with SDSS griz' (blue) and UKIDSS $Y J H K$ photometry (purple: WD, red: putative companion). Also shown are the SDSS spectrum (light grey) and an L4 spectrum scaled to the $K$-band magnitude of the putative secondary (dark grey). . $\quad 70$ 
2.17 SDSS finder image of WD $0922+006$. There is a hint of possible red contamination in the south east corner of the blue white dwarf. . . . . 71

2.18 UKIDSS $Y J H K$ images of WD $0922+006$. The red object clearly begins to dominate at longer wavelengths. . . . . . . . . . . .

2.19 SDSS J154431.47+060104.3 model spectrum (solid black) with SDSS griz' (blue) and UKIDSS $Y J H K$ photometry (red). Also shown are the SDSS spectrum (light grey) and a composite WD+T3 dwarf spectrum (dark grey). . . . . . . . . . . . . . . . .

2.20 SDSS J222551.65+001637.7 model spectrum (solid black) with SDSS griz' (blue) and UKIDSS $Y J H K$ photometry (red). Also shown are the SDSS spectrum (light grey) and a composite WD+L7.5 dwarf spectrum (dark grey). . . . . . . . . . . . . . . .

2.21 $K$-band image of SDSS J222551.65+001637.7 taken with SOFI on the NTT. The star is in the centre and is clearly elongated, possibly indicating the system is becoming resolved. . . . . . . . . . . .

2.22 WD 1248+161 model spectrum (solid black) with SDSS griz' (blue) and UKIDSS $H K$ (red) photometry. Also shown are the SDSS spectrum (light grey) and a composite WD+M8 dwarf spectrum (dark grey). The single $J$-band observation (purple) was classified as noise in the DR5 database and should be treated with caution. . . . . . . . . . . 
2.23 WD 1318+005 model spectrum (solid black) with SDSS griz' (blue), UKIDSS $Y J H K$ (red) and AAO IRIS2 $K$-band photometry (purple). Also shown are the SDSS spectrum (light grey) and a combined WD + $600 \mathrm{~K}$ blackbody model (dashed grey). . . . . . . . . . . . . .

2.24 SDSS J155720.77+091624.7 model spectrum (solid black) with SDSS griz' (blue) and UKIDSS $Y J H K$ photometry (red). Also shown are the SDSS spectrum (light grey), a combined WD+L4 dwarf model (dark grey), and a combined WD $+700 \mathrm{~K}$ blackbody model (dashed grey). . .

2.25 Predicted masses of companions based on the DUSTY models. The solid grey lines show the DUSTY models of various ages in Gyrs. . . . . . .

2.26 Distance verses projected orbital separation for a 2" (solid) and 1.2" (dashed) radius. . . . . . . . . . . . . . . . .

3.1 Model and observed spectrum of PHL 790 with composite models (dashed grey). 2MASS fluxes are also plotted (red). The dashed line indicates a region of very little atmospheric transmission. . . . . . . . . . . . . 103

3.2 Model subtracted spectrum of PHL 790B with an M4 star (grey) for comparison. The dashed line indicates a region of very little atmospheric transmission. ..................... 103

3.3 Model subtracted spectrum of PHL 790BB showing both the Na I and CO absorption lines. An M4 star is overplotted for comparison (red). . . 
3.4 Model and observed spectrum of GD 984 with composite models (dashed grey). 2MASS fluxes are also plotted (red). The dashed line indicates a region of very little atmospheric transmission. . . . . . . . . . . . . 104

3.5 Model subtracted spectrum of GD 984B in $J$ and $K$ bands, with an M3.5 star (grey) for comparison. The dashed line indicates a region of very little atmospheric transmission. . . . . . . . . . . . . . . . . 104

3.6 Model subtracted spectrum of GD 984B showing both the $\mathrm{Na}$ I and CO absorption lines. An M3.5 star is overplotted for comparison (red) . . . 104

3.7 Equivalent width of $\mathrm{NaI}$ as a function of effective temperature. Filled circles are the secondaries from this work with temperatures estimates based on spectral type. Also plotted are stars from Jones (1994) $(\times)$, Ivanov et al. (2004) (+) and Tappert et al. (2007) (o). . . . . . . . . . . 109

3.8 Near-IR two colour diagram for stars in this work divided in to the Wellhouse et al. (2005) regions delineating; (I) single white dwarfs, (II) white dwarfs with late-type main-sequence companions, (III) white dwarfs with lower mass, possible brown dwarf companions and (IV) white dwarfs contaminated with circumstellar material. Labelled as (1) WD 1415+132 (2) WD 1643+143 (3) WD 1412-049 (4) WD 1622+323 (5) WD 1305+018 (6) WD 2151-015 (7) WD 0419-487 (8) WD $1845+$ 019 (9) WD 0752 - 146 (10) WD 0131 - 163 (11)WD 0017+061. . . . 110 
4.1 Observed near-infrared spectrum of PG $1234+482$ scaled to the 2MASS $H$ flux. The predicted white dwarf model alone is shown by the solid line. The data are also compared to the white dwarf model combined with late M and early L dwarfs, all scaled to a distance of $144 \mathrm{pc}$ (Liebert, Bergeron \& Holberg 2005). The upper and lower grey spectra are PG 1234 + M8 and PG $1234+$ L2 spectra respectively. The $1.87 \mu \mathrm{m}$ H Paschen $\alpha$ emission line in the predicted white dwarf spectrum is due to non-LTE effects in the upper atmosphere. . . . . . . . . . . . . 118

4.2 2MASS and Spitzer IRAC $(4.5 \mu \mathrm{m}$ and $8.0 \mu \mathrm{m})$ photometric fluxes of PG 1234+482 with M9.5 and L1 fluxes scaled appropriately. The solid line is the white dwarf atmospheric model spectrum The symbols for the combined WD $+\mathrm{M}$ and $\mathrm{L}$ dwarf models do not represent the actual errors which can be seen in table $4.1 \ldots \ldots \ldots \ldots . \ldots$

5.1 Near-infrared acquisition images of PHL 5038 taken with Gemini + NIRI immediately prior to $H$ - and $K$-grism spectroscopy. Frames are 6 " across with north up and east left. The brown dwarf companion, PHL 5038B, is located 0.94" from A at position angle 293.2", as measured in the $K$ band image shown above. . . . . . . . . . . . . . . . . . . 129

5.2 Modelled (black) and observed (grey) $H K$ spectra of PHL 5038 A plotted with photometry as measured in the acquisition images. Note the detection of $\mathrm{Pa}_{\alpha}$ absorption. . . . . . . . . . . . . . 132 
5.3 Observed $H K$ spectrum (grey) of PHL 5038 B plotted with photometry as measured in the acquisition images. An L8 spectrum (2MASS 1632+1904) has been scaled to match the flux of the secondary and over-plotted (black) for comparison. . . . . . . . . . . . . . . . . . 132

5.4 Spatial geometry for the PHL 5038 binary system. The expected number of field brown dwarfs of spectral type L8-L9 existing in the same region, defined by the distance errors, to PHL 5038A is $\approx 3 \times 10^{-6}$. . . . . 135

6.1 Distribution of limits on unresolved companions to the 407 "single" DA white dwarfs (with well determined parameters) detected in UKIDSS DR5.143

6.2 Same as Figure 6.1 but plotted as a cumulative distribution. . . . . . . . 144

6.3 Cumulative distribution of distances to the 407 isolated DA white dwarfs in this work. . . . . . . . . . . . . . . . . . 146

6.4 Cumulative distribution of distances to the $204 \mathrm{DA}+\mathrm{dM}$ binaries in this work. . . . . . . . . . . . . . . . . . 147

6.5 Distance distribution of the white dwarfs presented in Farihi et al. (2005). The mean distance is shown as a solid line. . . . . . . . . . . . . 159

6.6 Distance distribution of the single DA white dwarfs present in the UKIDSS sample. The mean distance is shown as a solid line. . . . . . . . . . 160

6.7 Temperature distribution of the single DA white dwarfs present in the UKIDSS sample. The mean temperature is shown as a solid line. . . . . 163

7.1 The revised Large Are Survey footprint. . . . . . . . . . . . . . . 175 
7.2 Sky coverage of VISTA surveys, overlaid on a 2MASS image of the whole sky. . . . . . . . . . . . . . . . . . . 178

7.3 The $5 \sigma$ point source sensitivities of WISE and previous or planned allsky surveys. The planned wavelength range for the JWST is indicated. The dot size shows the planned sky coverage. GALEX is a small Explorer (SMEX), DPOSS is the groundbased Digital Palomar Observatory Sky Survey, ASTRO-F is the Japanese satellite, renamed Akari after launch on 22 Feb 2006, and Planck is the European CMB mission. Figure from www.astro.ucla.edu/wright/WISE/ . . . . . . . . . . . . 180

A.1 SDSS J003923.04+003534.7 model spectrum (black solid) with SDSS griz' (blue) and UKIDSS $H K$ photometry (red). Also shown are the SDSS spectrum (light grey) a composite WD+M9 spectrum (dark grey). 182

A.2 SDSS J032317.00-002612.7 model spectrum (black solid) with SDSS griz' (blue) and UKIDSS $J H K$ photometry (red). The extreme nearinfrared excess is likely caused by a bright background star. . . . . . . . 182

A.3 SDSS J034221.55+005345.6 model spectrum (black solid) with SDSS griz' (blue) and UKIDSS $Y J H K$ photometry (red). The extreme nearinfrared excess is likely caused by a bright background star. . . . . . . . 183

A.4 SDSS J085956.47+082607.5 model spectrum (solid black) with SDSS griz' (blue) and UKIDSS $Y H K$ photometry (red). Also shown are the SDSS spectrum (light grey) and a composite WD+M6 spectrum (dark

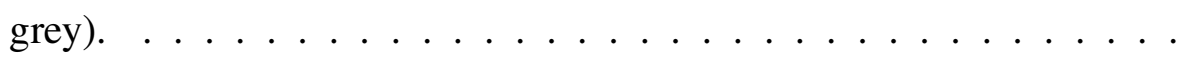


A.5 SDSS J090759.59+053649.7 model spectrum (solid black) with SDSS griz' (blue) and UKIDSS $Y J H K$ photometry (red). Also shown are the SDSS spectrum (light grey) and a composite WD+L4 spectrum (dark

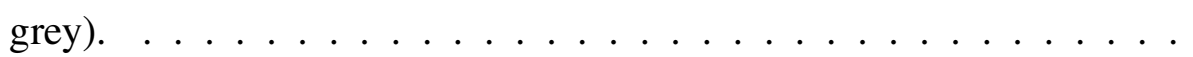

A.6 SDSS J092648.48+102828.8 model spectrum (solid black) with SDSS griz' (blue) and UKIDSS $Y J H K$ photometry (red). Also shown are the SDSS spectrum (light grey) and a composite WD+M8 spectrum. (dark grey)

A.7 SDSS J100259.88+093950.0 model spectrum (solid black) with SDSS griz' (blue) and UKIDSS $Y J H K$ photometry (red). Also shown are the SDSS spectrum (light grey) and a composite WD+L0 dwarf spectrum (dark grey). . . . . . . . . . . . . . . . .

A.8 SDSS J101642.93+044317.7 model spectrum (solid black) with SDSS griz' (blue) and UKIDSS $H K$ photometry (red). Also shown are the SDSS spectrum (light grey) and a composite WD+M3 dwarf spectrum (dark grey). . . . . . . . . . . . . . . .

A.9 SDSS J103220.19+011227.0 model spectrum (solid black) with SDSS griz' (blue) and UKIDSS $H K$ photometry (red). Also shown are the SDSS spectrum (light grey) and a composite WD+L2 dwarf spectrum (dark grey). . . . . . . . . . . . . . . . . 
A.10 SDSS J103448.92+005201.4 model spectrum (solid black) with SDSS griz' (blue) and UKIDSS $H K$ photometry (red). Also shown are the SDSS spectrum (light grey) and a composite WD+L5 dwarf spectrum

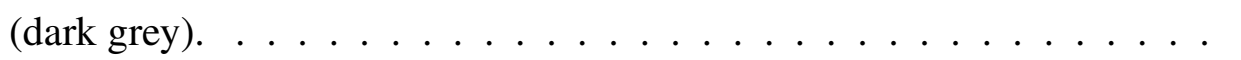

A.11 SDSS J103736.75+013912.2 model spectrum (solid black) with SDSS griz' (blue) and UKIDSS $H K$ photometry (red). Also shown are the SDSS spectrum (light grey) and a composite WD+M7 dwarf spectrum (dark grey). . . . . . . . . . . . . . .

A.12 SDSS J104933.58+022451.7 model spectrum (solid black) with SDSS griz' (blue) and UKIDSS $H K$ photometry (red). Also shown are the SDSS spectrum (light grey) and a composite WD+L3 dwarf spectrum

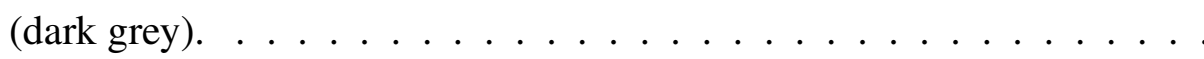

A.13 SDSS J105332.62+020126.2 model spectrum (solid black) with SDSS griz' (blue) and UKIDSS $H K$ photometry (red). Also shown are the SDSS spectrum (light grey) and a composite WD+L5 dwarf spectrum (dark grey). . . . . . . . . . . . . . . . . . . . . 188

A.14 SDSS J113416.09+055227.2 model spectrum (solid black) with SDSS griz' (blue) and UKIDSS $H K$ photometry (red). Also shown are the SDSS spectrum (light grey) and a composite WD+L3 dwarf spectrum (dark grey). . . . . . . . . . . . . . . . . . 188 
A.15 WD1155-011 model spectrum (solid black) with SDSS griz' (blue) and UKIDSS $Y J H K$ photometry (red). Also shown is a composite WD+M7 dwarf spectrum (dark grey). . . . . . . . . . . . . . . . 189

A.16 SDSS J132925.21+123025.4 model spectrum (solid black) with SDSS griz' (blue) and UKIDSS $Y J H K$ photometry (red). Also shown are the SDSS spectrum (light grey) and a composite WD+M7 dwarf spectrum (dark grey). . . . . . . . . . . . . . . . .

A.17 SDSS J220841.63-000514.5 model spectrum (solid black) with SDSS griz' (blue) and UKIDSS $Y J H K$ photometry (red). Also shown are the SDSS spectrum (light grey) and a composite WD+L1 dwarf spectrum (dark grey). . . . . . . . . . . . . . . . . . . . . 190

A.18 PHL 5038 model spectrum (solid black) with SDSS griz' (blue) and UKIDSS $Y J H K$ photometry (red). Also shown are the SDSS spectrum (light grey) and a composite WD+L8 dwarf spectrum (dark grey). . . . 190

A.19 SDSS J233345.97-000843.0 model spectrum (solid black) with SDSS griz' (blue) and UKIDSS $H K$ photometry (red). Also shown are the SDSS spectrum (light grey) and a composite WD+M7 dwarf spectrum (dark grey). . . . . . . . . . . . . . . .

B.1 The observed spectrum of V* RR Cae, modelled with a blackbody, with composite models (dashed grey). 2MASS fluxes are also plotted (red). The dashed line indicates a region of very little atmospheric transmission. 197 
B.2 Model subtracted spectrum of V* RR Cae B with an M4 star for comparison. The dashed line indicates a region of very little atmospheric

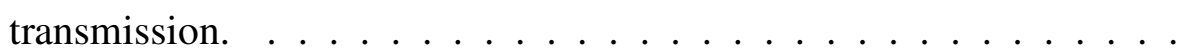

B.3 Model subtracted spectrum of $\mathrm{V}^{*} \mathrm{RR}$ Cae showing both the $\mathrm{Na}$ I and $\mathrm{CO}$ absorption lines. An M4 star is overplotted for comparison (red) . . .

B.4 Model and observed spectrum of EGGR 57 with composite model (dashed grey). 2MASS fluxes are also plotted (red). The dashed line indicates a region of very little atmopsheric transmission. . . . . . . . . . . . .

B.5 Model subtracted $H K$ spectrum of EGGR 57B with an M6 star (grey) for comparison. The dashed line indicates a region of very little atmospheric

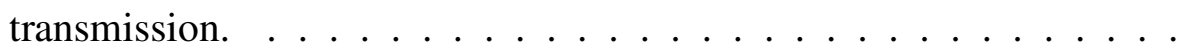

B.6 Model subtracted spectrum of EGGR 57 showing both the $\mathrm{Na}$ I and $\mathrm{CO}$ absorption lines. An M6 star is overplotted for comparison (red) . . . .

B.7 Model and observed spectrum of PG $1305+018$ with composite models (dashed grey). 2MASS fluxes are also plotted (red). The dashed line indicates a region of very little atmopsheric transmission. . . . . . . .

B.8 Model subtracted spectrum of PG 1305+018B with an M4 star (grey) for comparison. The dashed line indicates a region of very little atmospheric

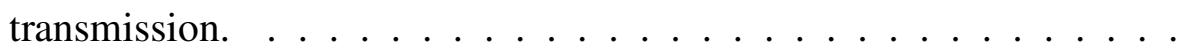

B.9 Model subtracted spectrum of PG $1305+018$ showing both the $\mathrm{Na}$ I and CO absorption lines. An M4 star is overplotted for comparison (red) . . 
B.10 Model and observed spectrum of PG 1412-049 with composite models (dashed grey). 2MASS fluxes are also plotted (red). The dashed line indicates a region of very little atmospheric transmission. . . . . . . . 200

B.11 Model subtracted spectrum of PG 1412-049B with an M1 star (grey) for comparison. The dashed line indicates a region of very little atmospheric transmission. ........................ 200

B.12 Model subtracted spectrum of PG 1412-049 showing both the $\mathrm{Na}$ I and CO absorption lines. An M1 star is overplotted for comparison (red) . . 200

B.13 Model and observed spectrum of Feige 93 with composite models (dashed grey). 2MASS fluxes are also plotted (red). The dashed line indicates a region of very little atmospheric transmission. . . . . . . . . . . . . 201

B.14 Model subtracted $J$ and $K$ spectra of Feige 93 B with an M3.5 star (grey) for comparison. The dashed line indicates a region of very little atmospheric transmission. . . . . . . . . . . . . . . . 201

B.15 Model subtracted spectrum of Feige 93 showing both the $\mathrm{Na}$ I and $\mathrm{CO}$ absorption lines. An M3.5 star is overplotted for comparison (red) . . . 201

B.16 Model and observed spectrum of PG $1622+323$ with composite models (dashed grey). 2MASS fluxes are also plotted (red). The dashed line indicates a region of very little atmospheric transmission. . . . . . . . . 202

B.17 Model subtracted $J$ and $K$ spectra of PG $1622+323 \mathrm{~B}$ with an M0.5 star (grey) for comparison. The dashed line indicates a region of very little atmospheric transmission. . . . . . . . . . . . . . 202 
B.18 Model subtracted spectrum of PG $1622+323$ showing both the $\mathrm{Na}$ I and CO absorption lines. An M0.5 star is overplotted for comparison (red) . 202

B.19 Model and observed spectrum of PG $1643+143$ with composite models (dashed grey). 2MASS fluxes are also plotted (red). The dashed line indicates a region of very little atmospheric transmission. . . . . . . . 203

B.20 Model subtracted spectrum of PG 1643+143B with an M2 star (grey) for comparison. The dashed line indicates a region of very little atmospheric

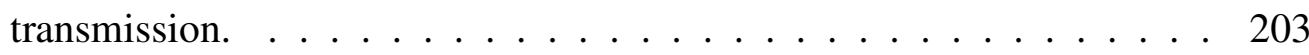

B.21 Model subtracted spectrum of PG $1643+143$ showing both the $\mathrm{Na}$ I and CO absorption lines. An M2 star is overplotted for comparison (red) . . 203

B.22 Model and observed spectrum of Lanning 18 with composite models (dashed grey). 2MASS fluxes are also plotted (red). The dashed line indicates a region of very little atmospheric transmission. . . . . . . . . 204

B.23 Model subtracted $J$ and $K$ spectra of Lanning $18 \mathrm{~B}$ with an M4 star (grey) for comparison. The dashed line indicates a region of very little atmospheric transmission. . . . . . . . . . . . . . . 204

B.24 Model subtracted spectrum of Lanning 18 showing both the $\mathrm{Na}$ I and $\mathrm{CO}$ absorption lines. An M4 star is overplotted for comparison (red) . . . . 204

B.25 The observed spectrum of GJ 4236, modelled with a blackbody, with composite models (dashed grey). 2MASS fluxes are also plotted (red). The dashed line indicates a region of very little atmospheric transmission. 205 
B.26 Model subtracted spectrum of GJ 4236B with an M8 star (grey) for comparison. The dashed line indicates a region of very little atmospheric

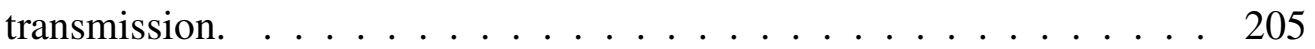

B.27 Model subtracted spectrum of GJ 4236 showing both the $\mathrm{Na}$ I and CO absorption lines. An M8 star is overplotted for comparison (red) . . . . 205 


\section{List of Tables}

1.1 The Harvard spectral classification scheme . . . . . . . . . . . . . 10

1.2 Primary White Dwarf Spectral Classifications . . . . . . . . . . 11

2.1 Number of each white dwarf spectral type detected in the cross correlation of UKIDSS LAS DR5 $\ldots \ldots \ldots \ldots$

2.2 Near infra-red magnitudes of known white dwarfs with unresolved brown dwarf companions . . . . . . . . . . . . . . . . . . . 44 44

2.3 Near infra-red magnitudes of known white dwarfs with circumstellar debris disks $\ldots \ldots \ldots \ldots \ldots \ldots \ldots \ldots \ldots$

2.4 The combinations of UKIDSS data available for the 1161 SDSS DR4 white dwarfs. . . . . . . . . . . . . . . 46 46

2.5 UKIDSS $Y J H K$ magnitudes of the candidate white dwarfs showing near-infrared excesses. . . . . . . . . . . . . .

2.6 Physical parameters of the candidate white dwarfs showing near-infrared excesses. Temperatures and surface gravities are from Eisenstein et al. (2006) unless otherwise stated. 
2.7 The number density $n$ of spectral types ranging from M3-T8 V. Table from Caballero, Burgasser \& Klement (2008) _ . . . . . . . . .

2.8 Summary of proposed sources for the near-infrared excesses identified in UKIDSS. For the resolved systems, the probability of a chance alignment is given. Further information on systems with a putative companion is given in Table $2.9 \ldots \ldots \ldots \ldots \ldots \ldots \ldots$

2.9 Table of results for SDSS white dwarfs with NIR excess indicative of a companion: most likely companion spectral types and mergedClass (MC) stats indicating the possibility that a system is a widely separated $(+1)$ or a close detached binary $(-1$ or -2$) \ldots \ldots \ldots \ldots$

3.1 Summary table of observations for each white dwarf in the sample. . . .

3.2 Summary of the physical parameters for each white dwarf used in this work. The first reference refers to the temperature and surface gravity, the second to the $V$-band magnitude, and the third to the distance. In cases where no reference has been provided, the distance has been derived by parallax measurements or from the Bloecker evolutionary mod-

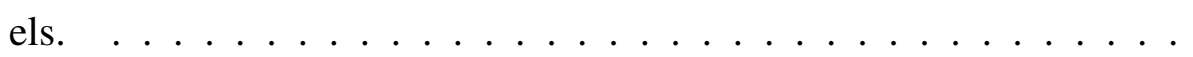

3.3 Summary details of the white dwarfs studied in this work, including nearIR magnitudes for each star obtained from the 2MASS All-Sky Point Source Catalogue. . . . . . . . . . . . . . . . . . . . . . . 100 
3.4 Summary of results to the near-infrared spectroscopic identification giving the spectral type of each companions. Also shown are previous photometric estimates and projected orbital distances. . . . . . . . . . 105

3.5 Equivalent width of the $\mathrm{NaI}$ lines at $2.2 \mu \mathrm{m}$ for each companion . . . . 107

4.1 Photometric fluxes in mJy of combined PG 1234 (Predicted) + M and L dwarfs compared to those of PG 1234 (Observed) . . . . . . . . . . . 117

4.2 The lowest mass detached companions to white dwarfs. . . . . . . . . . 124

4.3 The lowest mass companions to CV's . . . . . . . . . . . . . . . 124

5.1 Near-Infrared photometry of the PHL 5038 binary system and nearby calibration star. . . . . . . . . . . . . . . 126

5.2 Spectral indices as measured for PHL 5038 B, L8, L9 and T0 fields dwarfs (Burgasser et al. 2006). . . . . . . . . . . . . . . 133

5.3 MS progenitor masses $\left(\mathrm{M}_{\mathrm{MS}}\right)$ and ages $\left(\mathrm{t}_{\mathrm{MS}}\right)$, WD cooling ages $\left(\mathrm{t}_{\mathrm{C}}\right)$, total age $\left(t_{\text {total }}\right)$, and MS age as a fraction of WD cooling age for PHL 5038A. 136

6.1 Number of each spectral type placed as a lower limit to the 407 apparently single DA white dwarfs in UKIDSS DR5. . . . . . . . . . . . . . 142

6.2 Actual number and effective number of detections for DA white dwarf $+\mathrm{dL}$ binaries in the UKIDSS DR5 sample based on sensitivity estimates. 154 
6.3 Actual number and effective number of detections for DA white dwarf + dT binaries in the UKIDSS DR5 sample based on sensitivity estimates. No candidate companions later than spectral type T3 were detected. . . 155

6.4 Actual number and effective number of detections for DA white dwarf + brown dwarf binaries in the UKIDSS DR5 sample based on sensitivity estimates. No candidate companions later than spectral type T3 were detected. . . . . . . . . . . . . . . 156

C.1 Physcial parameters and UKIDSS data for SDSS DR4 DA white dwarfs in this work. . . . . . . . . . . . . . . . 207

C.2 Physcial parameters and UKIDSS data for SDSS DR4 DB white dwarfs in this work. . . . . . . . . . . . . . . . . 227

C.3 Physical parameters and UKIDSS data for McCook \& Sion DA white dwarfs in this work. . . . . . . . . . . . . . . . 228 


\section{Chapter 1}

\section{Introduction}

\subsection{White Dwarfs}

\subsubsection{Overview}

White dwarfs are the endgame of stellar evolution for stars with a mass up to $\leq 8 M_{\odot}$ (Weidemann 1987, Casewell et al. 2009). Approximately 98\% (Wood 1992) of all stars will end their lives as white dwarfs, making these stellar remnants the most common fate of the stars in our universe. White dwarfs owe their stability to their compact size and extreme density. The peak of the white dwarf mass distribution lies at $0.6 M_{\odot}$ (Weidemann $\&$ Koester 1984), with a range of masses starting from $\approx 0.17 M_{\odot}$ (Kilic et al. 2007b) up to a maximum mass of $\approx 1.4 M_{\odot}($ Chandrasekhar 1931).

This chapter introduces the topic of white dwarfs, their internal and atmospheric structure, how they are classified, their previous and continued evolution, and I discuss the more recent discoveries that reveal these stellar corpses can harbour more complex solar 
systems of their own.

\subsubsection{Discovery}

The first white dwarf was discovered in the triple-star system 40 Eridani, consisting of the main-sequence star 40 Eri A, orbited by the closer binary of the white dwarf 40 Eri B and a main-sequence red dwarf 40 Eri C.

The binary 40 Eri BC was first discovered by Friedrich W. Herschel on January $31^{\text {st }}$ 1783. In 1910 Henry N. Russel, Edward C. Pickering and Williamina Fleming obtained a spectrum of 40 Eri B and found the spectral class to be that of an A-type star. This was a surprising result, as the luminosity of 40 Eri B suggested a much cooler M-type and would have to be 1000 times brighter to be classed as one of these hot white stars. Not long afterwards, in 1915, Walter S. Adams obtained a spectrum of Sirius B, and found that it also was of spectral type A (Adams 1915), the same as its much more luminous companion Sirius A. This placed both 40 Eri B and Sirius B in the lower left hand corner of the Hertzsprung-Russel diagram, a previously unoccupied area (Figure 1.1). Thus, both 40 Eri B and Sirius B both had a low luminosity whilst still having a high temperature. This raised problems for astrophysicists, as hot stars were supposed to be more luminous than cool stars of the same size. The only logical explanation was that these stars must be 100 times smaller than the Sun, which in turn implied an enormous internal density. No physical laws existed at the time to explain these conditions, and hence the existence of these strange objects. 


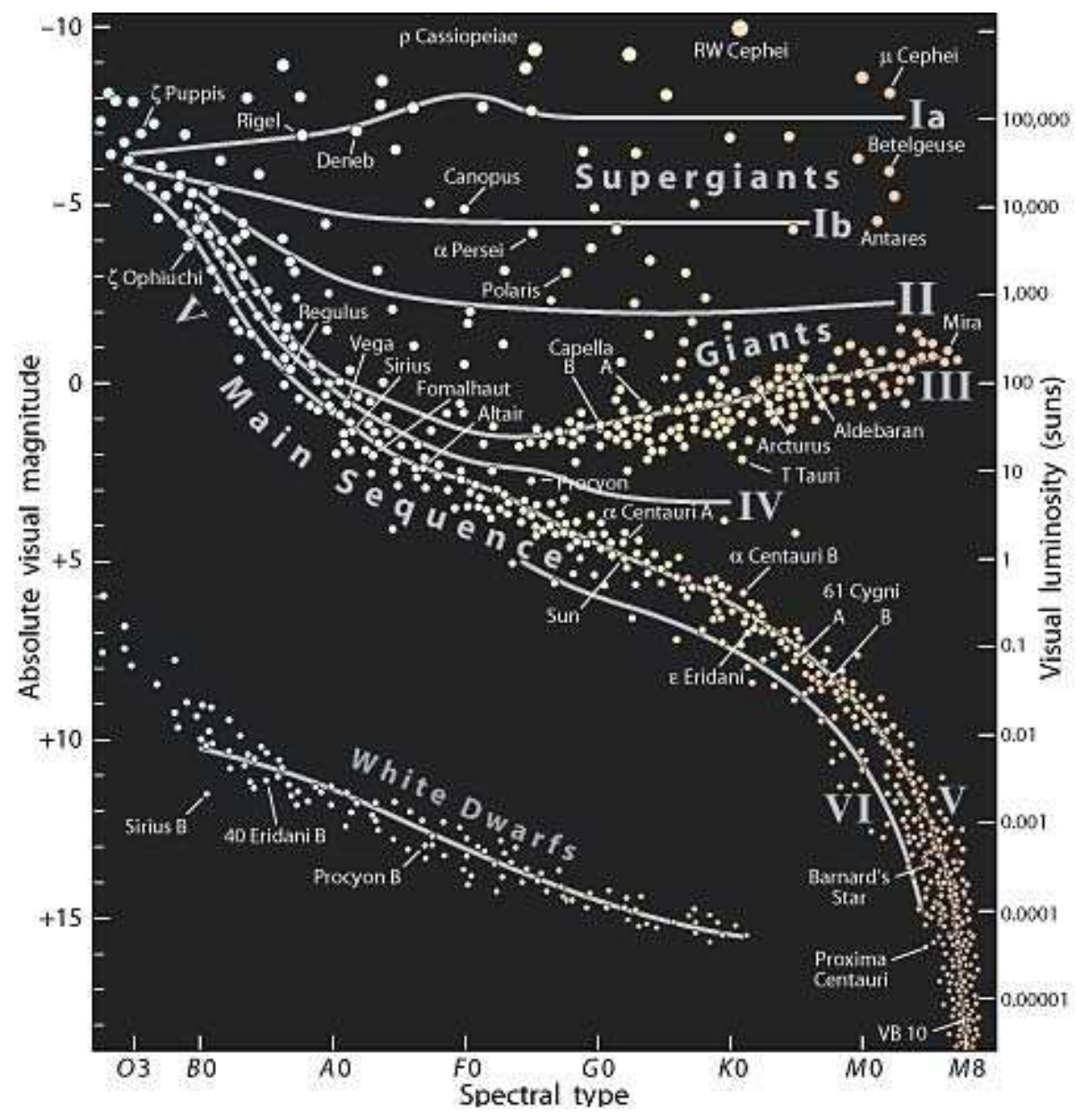

FIGURE 1.1. Hertzsprung-Russel Diagram showing the positions of 40 Eridani B and Sirius B, now known to be white dwarfs. 
In 1917 Adriaan Van Maanen discovered Van Maanen's Star, the first isolated white dwarf. It wasn't until 1922 that Willem Luyten coined the phrase "white dwarf" to describe these exotic objects, a term later popularised by Arthur S. Eddington. By the 1950s over 100 white dwarfs had been found, and currently over 10,000 have been identified by the Sloan Digital Sky Survey, and over 2000 by various other surveys.

\subsubsection{White Dwarf Structure}

In 1926 Enrico Fermi and Paul Dirac developed the quantum statistical theory of an electron gas, resulting in major advances in the understanding of a white dwarf's internal structure, and finally providing a solution to the erstwhile luminosity-radius problem. According to Fermi-Dirac statistics, fermions (particles with $1 / 2$ integer spin e.g. protons and electrons) occupy discrete energy levels, and according to the Pauli exclusion principle, no two fermions with the same spin can occupy the same quantum energy state (Hoddeson \& Baym 1980). As a fermion gas is compressed and the particle density increases, fermions will be forced to occupy certain quantised energy levels. A fermion gas where all the energy levels are filled below a threshold value, called the Fermi energy, is known as a degenerate fermion gas. Once in this state, the fermions resist further compression, a force known as degeneracy pressure. In 1926 Sir Ralph Fowler showed that electron degeneracy pressure could theoretically support a white dwarf against gravitational collapse (Fowler 1926).

In 1931 Subrahmanyan Chandrasekhar combined relativity and quantum mechanics to 
successfully produce the first basic equations of white dwarf structure. He equated gravitational potential energy against electron degeneracy pressure to show that for a degenerate object, $R \propto M^{-1 / 3}$. This relationship shows that the more massive the object, the smaller its radius will become. He then predicted the maximum non-rotating mass that can be supported against gravitational collapse by electron degeneracy pressure to be $\approx 1.4 M_{\odot}$ for objects with solar metallicity (Chandrasekhar 1931). Thus, this is the maximum possible mass of a white dwarf, and is known as the Chandrasekhar limit.

A typical white dwarf is composed of an electron degenerate core which makes up for $99.99 \%$ of the overall mass, and a thin non-degenerate atmosphere composed of hydrogen or helium. The core is primarily composed of carbon and oxygen, as these are the products leftover from the helium burning phase of the progenitor (Section 1.1.5), and is in a plasma state consisting of ions and degenerate electrons. The electron degeneracy pressure is very weakly dependent on temperature and so a white dwarf will continue to cool at a roughly constant radius throughout the majority of its lifetime.

As the electrons already occupy their lowest possible energy state they cannot contribute to the cooling of the white dwarf. Instead, it is the non-degenerate ions that provide the thermal energy required to maintain the star's luminosity. Over time these ions will condense from a gas to a liquid state and eventually, between $4000-10,000 \mathrm{~K}$, they crystallise into a solid. Winget (1995) suggested that pulsating white dwarfs could provide a testing ground for the crystallisation theory, and Metcalfe et al. (2004) used this theory to calculate that $\approx 90 \%$ of the white dwarf BPM 37093 had already crystallized. Eventually a 
white dwarf will cool until it no longer emits enough light to be visible, and will end its life as a cold, non-radiating black dwarf.

Unless a white dwarf accretes matter from a companion star or other source then its only source of energy is this internal heat which cannot be replenished. As a white dwarf has only a small surface area from which to radiate, it follows that it should cool gradually, remaining relatively hot for a large period of time. For temperatures $>100,000 \mathrm{~K}$ the main contributor to cooling is actually neutrino loss, with approximately twice as much energy being lost through this process as opposed to radiation. However, at $T \approx 15,000 \mathrm{~K}$ the contribution from neutrino flux has fallen to $<1 \%$, and radiation now dominates the cooling process.

Since a white dwarf's energy will mostly be lost through radiation, it also follows that the rate of cooling will slow with time. For example, it can be seen in Bergeron et al. (1997) that a hydrogen atmosphere carbon core white dwarf with a typical mass of $0.6 M_{\odot}$ will take $\approx 1.5$ Gyr to cool to $\approx 7000 \mathrm{~K}$. Cooling a further $1000 \mathrm{~K}$ then takes another $0.8 \mathrm{Gyr}$, around a third of the white dwarf's total cooling age. A further decreases of only $500 \mathrm{~K}$ then takes a considerably longer 1.1 Gyr. A graph of age verses temperature for both a $0.6 M_{\odot}$ and $1.0 M_{\odot}$ white dwarf is shown in Figure 1.2.

\section{Magnetic Fields}

A magnetic field with a surface strength of $\approx 1,000,000 \mathrm{G}(100 \mathrm{~T})$ was first predicted to occur in white dwarfs by Blackett (1947), due to an uncharged, rotating body producing a 


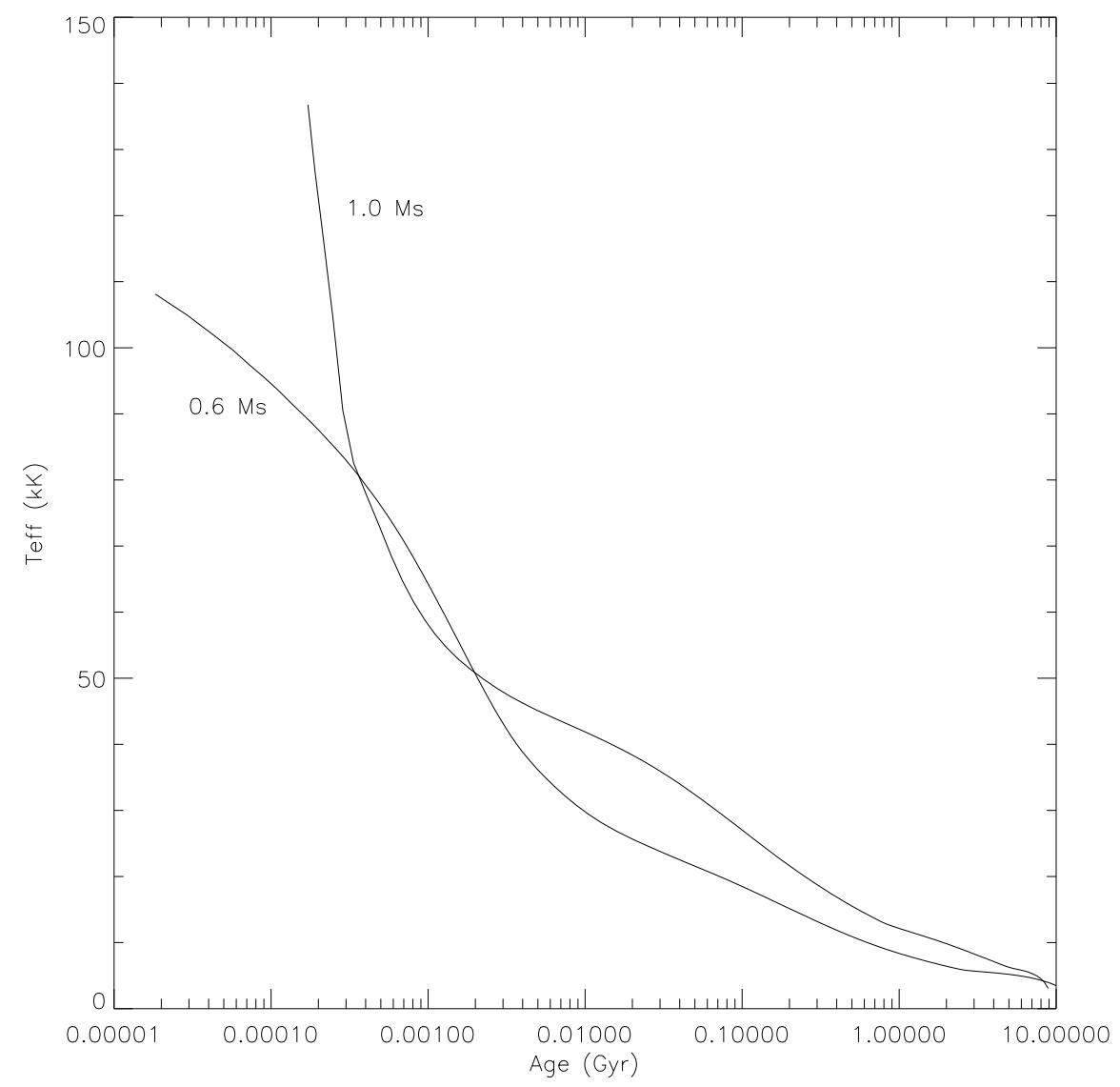

FIGURE 1.2. Age vs. effective temperature for both a $0.6 M_{\odot}$ and $1.0 M_{\odot}$ white dwarf showing how cooling slows with white dwarf age. Curves are based on the models of Wood (1994). 
magnetic field proportional to its angular momentum. However, by the 1950's Blackett's law had been widely refuted. A decade later it was proposed that a white dwarf may have a magnetic field due the conservation of total surface magnetic flux as a mainsequence star evolved into a white dwarf. As a consequence the strength of the magnetic field would increase by a factor of $\mathrm{R}^{2}$ as the radius of the star shrank in size. Thus a progenitor with a magnetic field of $100 \mathrm{G}$ would become a white dwarf with a magnetic field of $1,000,000 \mathrm{G}$, as the star's radius decreased by a factor of 100 . The first white dwarf to be discovered with a magnetic field was GJ742, confirmed by the detection of circularly polarised light. Since then $>100$ magnetic white dwarfs have been discovered and it has been estimated that $\approx 10 \%$ of white dwarfs have a magnetic field in excess of $1,000,000 \mathrm{G}$.

\subsubsection{White Dwarf Classification}

Although the core of a white dwarf is primarily composed of carbon and oxygen, spectroscopy has revealed that the atmosphere is dominated by either hydrogen or helium. Schatzman (1958) deduced that this atmospheric purity was due to the high surface gravity of white dwarfs, which causes the heaviest elements to rapidly sink out of the atmosphere leaving only the lightest, $\mathrm{H}$ or $\mathrm{He}$, behind.

The first attempt to spectrally classify white dwarfs was made by Kuiper (1941). Almost 20 years later Greenstein (1960) created a new system that continued to be used for the following 20 years. This was based on a previous scheme by Luyten (1952), and made 
use of the current standard Harvard spectral classification scheme (Table 1.1). All white dwarf spectral types were prefixed with an uppercase $\mathrm{D}$, for degenerate. A second letter was then assigned based on the stars spectroscopic resemblance to main sequence stars. Hence, DA white dwarfs had spectra dominated by strong hydrogen lines similar to that seen in main-sequence A-type stars. However, due to the growing diversity of white dwarf spectra, the appearance of certain hybrids, and the inability to distinguish between hot and cool stars, it was soon realised that a new classification system was needed.

Thus, a new system was proposed by Sion et al. (1983) which is still in use today. The uppercase D was retained, with a second uppercase letter assigned based on the most dominant element present in the star's spectrum. More than one letter may be used if more than one element is present, but they always appear in order of dominance. These can then be followed by an optional letter representing any secondary features present in the spectrum, followed by a temperature index calculated by dividing 50,400 by the star's effective temperature. The classification scheme is shown in Table 1.2. For example, a white dwarf with polarised light due to a magnetic field, an effective temperature of $15,000 \mathrm{~K}$, and a spectrum dominated by $\mathrm{H}$ lines could be given the classification of DAP3. 
Table 1.1. The Harvard spectral classification scheme

\begin{tabular}{|c|c|c|c|}
\hline $\begin{array}{l}\text { Spectral } \\
\text { Type }\end{array}$ & $\begin{array}{l}\text { Temperature } \\
\qquad[\mathrm{K}]\end{array}$ & Colour & Characteristics \\
\hline $\mathrm{O}$ & $>25,000$ & Blue & $\begin{array}{l}\text { Strong HeII absorption (sometimes emission) lines } \\
\text { HeI absorption lines becoming stronger }\end{array}$ \\
\hline $\mathrm{B}$ & $11,000-25,000$ & Blue-White & $\begin{array}{l}\text { HeI absorption lines strongest } \\
\text { HI (Balmer) absorption lines becoming stronger }\end{array}$ \\
\hline A & $7,500-11,000$ & White & $\begin{array}{l}\text { HI (Balmer) absorption lines strongest } \\
\text { CaII absorption lines becoming stronger }\end{array}$ \\
\hline $\mathrm{F}$ & $6,000-7,500$ & Yellow-White & $\begin{array}{l}\text { HI (Balmer) absorption lines becoming weaker } \\
\text { CaII absorption lines becoming stronger } \\
\text { Neutral metal absorption lines (FeI, CrI) }\end{array}$ \\
\hline $\mathrm{G}$ & $5,000-6,000$ & Yellow & $\begin{array}{l}\text { CaII absorption lines becoming stronger } \\
\text { Neutral metal absorption lines becoming stronger }\end{array}$ \\
\hline $\mathrm{K}$ & $3,500-5,000$ & Orange & $\begin{array}{l}\text { CaII absorption lines strongest } \\
\text { Spectra dominated by neutral metal absorption lines }\end{array}$ \\
\hline M & $<3,500$ & Red & $\begin{array}{l}\text { Spectra dominated by molecular absorption bands, especially TiO } \\
\text { Neutral metal absorption lines remain strong }\end{array}$ \\
\hline
\end{tabular}


Table 1.2. Primary White Dwarf Spectral Classifications

\begin{tabular}{ccl}
\hline \hline Class & $\begin{array}{c}\text { Approx. Temperature } \\
\text { Range }(\mathrm{K})\end{array}$ & Spectral Characteristics \\
\hline H-rich & & \\
DA & $6,000-100,000$ & Balmer lines only, no He or metal features \\
DAO & $>45,000$ & Balmer lines and weak HeII features \\
He-rich & & \\
DO & $45,000-100,000$ & Strong HeII lines, some HeI present \\
DB & $12,000-30,000$ & HeI lines, no H or metals \\
DBA & $12,000-30,000$ & HeI lines and weak Balmer lines present \\
Cool WDs & & \\
DQ & $6,000-12,000$ & C features (atomic or molecular) \\
DZ & $<6,000$ & Metal lines only, no H or He \\
DC & $<6,000$ & Featureless continuum (no lines deeper than 5\%) \\
\hline \hline Additonal & & Secondary Feature \\
\hline P & & Magnetic with polarisation \\
H & & Magnetic with no detectable polarisation \\
E & & Emission lines present \\
V & & Variable \\
d & & Debris Disk \\
\hline \hline
\end{tabular}

\subsubsection{White Dwarf Formation}

If a star's initial mass is $\leq 8 M_{\odot}$ then its fate will be to become a white dwarf (Weidemann \& Koester 1983). A main-sequence star provides the necessary energy to prevent gravitational collapse by burning hydrogen in its core, a state called hydrostatic equilibrium. The more mass a star has, the higher its gravity and hence more massive stars burn hydrogen quickly and so have relatively short lives compared to those of their lower mass cousins. During this phase the star will burn hydrogen at a roughly constant radius and is known as a main-sequence (MS) star. However, all stars will eventually run out of their hydrogen fuel, causing the core to collapse (Schönberg \& Chandrasekhar 1942). 
This contraction increases the temperature within the core as well as the surrounding material. How the star then evolves, depends on its initial mass.

For very low mass stars with initial mass $<0.5 M_{\odot}$ the core will never become hot enough to undergo helium fusion. Such stars are expected to eventually evolve into white dwarfs with helium cores. However, as this process has a timescale larger than the age of the universe (Laughlin et al. 1997), observed helium core white dwarfs are expected to have evolved through mass transfer in a binary system (e.g. Liebert et al. 2004).

For a star of intermediate initial mass between $\approx 1.1-8 M_{\odot}$ a typical evolutionary path on the Hertzsprung-Russell diagram is shown in Figure 1.3. After the depletion of hydrogen, the subsequent core contraction increases the temperature of both the core and surrounding material. Hydrogen burning continues in the surrounding shell, providing $\approx 100$ times the previous luminosity and causing a steady increase in the mass of the helium core. The core size eventually reaches the Schönberg-Chandresekhar limit, and again begins to collapse, resulting in the release of gravitational potential energy. This forces the outer envelope of the star to expand by a factor between 100 to 1000 , decreasing the surface luminosity and effective temperature. The star now moves onto the red giant branch (RGB). This decrease in effective temperature causes the envelope to become convective, transporting more hydrogen to the burning shell.

The continued shell burning of hydrogen continually adds helium to the contracting core until the density and temperature become high enough to ignite helium burning. This occurs via the triple $-\alpha$ process, resulting in the production of carbon and oxygen. An 


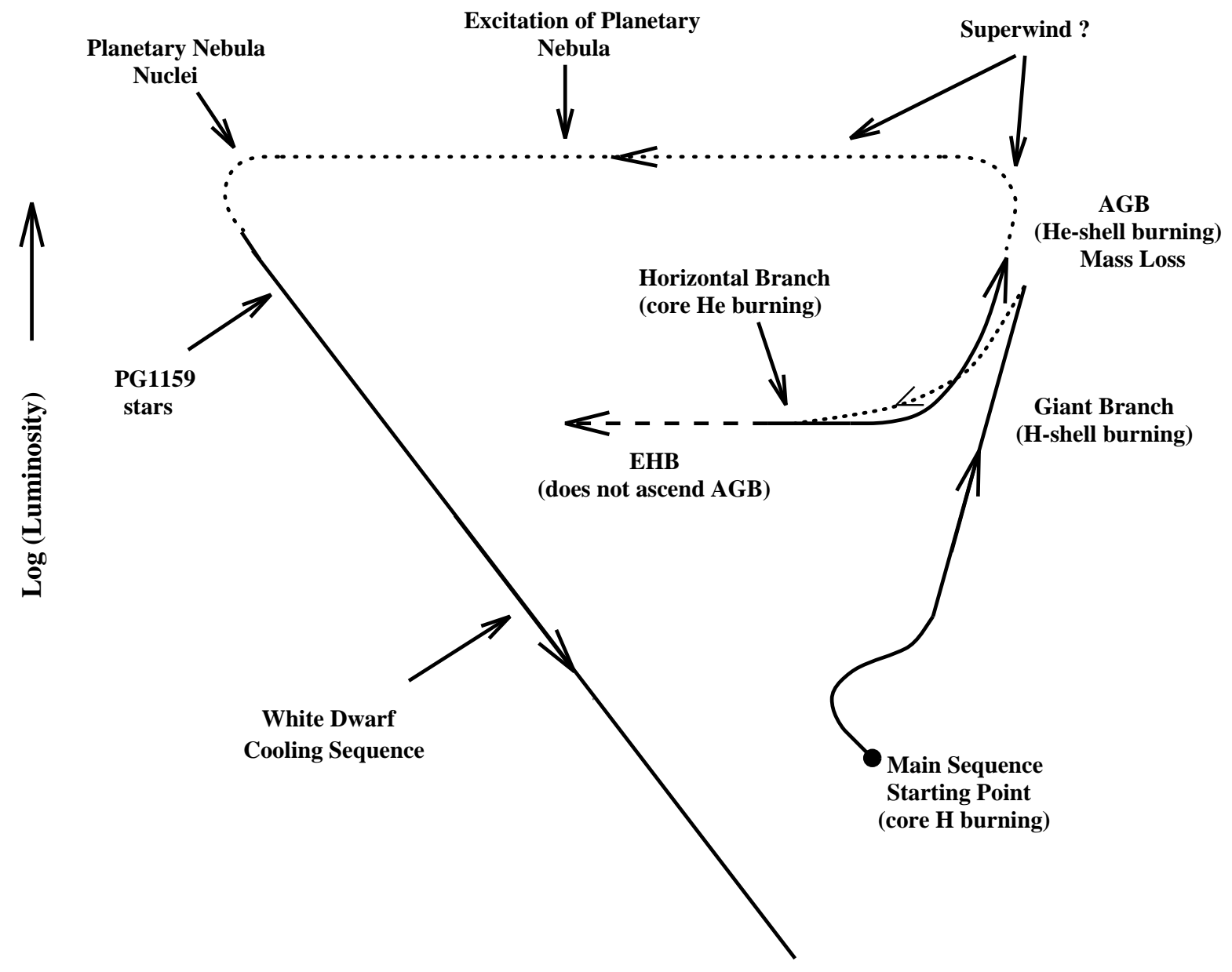

$\leftarrow \quad \log$ (Effective Temperature)

FIGURE 1.3. The Hertzsprung-Russell diagram, showing the evolutionary paths of stars from the main sequence to the white dwarf cooling sequence (reproduced from Marsh 1995). 
interesting difference occurs at this stage at around the $2 M_{\odot}$ boundary. For stars with initial mass $<2 M_{\odot}$ the core contraction is ongoing up to the tip of the RGB, producing a highly electron degenerate core. The degeneracy pressure is sufficient enough to stop the collapse of the most central matter. Eventually the temperature is reach at which helium can begin to fuse $\left(\approx 100 \times 10^{6} \mathrm{~K}\right)$ The electron degeneracy pressure is very weakly temperature dependant which is in opposition to the high temperature dependency of the triple $-\alpha$ process. Therefore, once the temperature reaches $100 \times 10^{6} \mathrm{~K}$ and helium ignition occurs, the temperature rapidly increases as the degenerate matter is a good thermal conductor. This increases the helium fusion rate and expands the reaction region. However, the volume does not increase and nor does the pressure, so the core does not expand in order to stabilise. Thus, for a few seconds, there is a runaway reaction increasing the star's energy production to $\approx 100 \times 10^{9}$ the normal amount. That is until the thermal pressure once again dominates due to the rising temperature, eliminating the degeneracy. This effect is known as the helium flash.

With the onset of helium burning the core once again expands, slowing the rate of helium and shell hydrogen burning. The envelope contracts rapidly, and the effective temperature begins to increase. At this stage the star is now moving along the horizontal branch of the HR diagram, characterised by a helium burning core and a hydrogen burning shell. Many stars will develop pulsations at this stage due to instabilities in their outer envelopes.

Once the supply of helium is exhausted the entire star contracts. The increase in temper- 
ature associated with the contraction of the core results in a thick helium burning shell. As the core contraction continues, the helium shell narrows and strengthens, resulting in the expansion and cooling of the outer layers. This in turn causes the hydrogen shell to temporarily "switch off". Once the star reaches the Hayishi track, the star moves upwards onto the asymptotic giant branch (AGB). During the AGB phase the helium burning shell turns on and off periodically in a series of helium shell flashes. This occurs because helium is being dumped onto the shell by the above hydrogen burning layer. As the mass of the helium increases it starts to become degenerate and, upon the ignition of helium burning, the degeneracy is lifted in a analogous manor to that described previously. This in turn drives the hydrogen shell outwards, where upon it cools and turns off again. The cycle then repeats.

As the star moves up the AGB, a carbon-oxygen core is steadily growing in mass. For stars with initial mass $>8 M_{\odot}$ this will eventually lead to the onset of carbon burning and set the star on an evolutionary path to becoming a neutron star or black hole. As the density of the core increases, electron degeneracy pressure begins to dominate. Mass loss will begin to occur at this stage as the result of poorly understood thermal pulsations, ejecting the outer atmosphere. It has also been suggested that a superwind will develop towards the end of the mass loss phase, the reasons for which also remain unclear. The expanding gas shell surrounding a white dwarf progenitor is known as a planetary nebula (PN). Red giants can support a wind with a mass loss rate of $\approx 10^{-6} M_{\odot} \mathrm{yr}^{-1}$, but it has been estimated that at least a rate of $10^{-5} M_{\odot} \mathrm{yr}^{-1}$ is needed to create the observed PN (Renzini \& Voli 1981). There have been extensive studies 
into the difficulties surrounding post-AGB mass loss (Iben \& Renzini 1983, Marigo et al. 1996, Maraston 1998, Maraston 2005). However, the relationships between the fundamental parameters (Luminosity, mass, metallicity and mass loss rate) are still poorly understood (González-Lópezlira et al. 2010).

Inside the expanding shell of gas, the carbon-oxygen core continues to contract at constant luminosity, moving the star horizontally and to the left on the HR diagram. During this phase, the stars temperature will increase to $>100,000 \mathrm{~K}$ and the surface gravity by a factor $>10^{4}$. At a temperature of $\approx 30,000 \mathrm{~K}$, ultraviolet photons are absorbed by the nebula gas, causing the atoms to become ionized. As the electrons drop to lower energy levels they emit light at visible wavelengths, creating the glowing ring of gas characteristic of a PN. The hydrogen and helium shells of the central star are eventually extinguished, and the star now cools to become a hot white dwarf star. Within $\approx 100,000$ years the PN itself has dissipated into the surrounding interstellar gas. Compared with the evolutionary timescale of the star, the PN phase is very shortlived.

\section{The Initial Final Mass Relationship}

The Initial Final Mass Relationship (IFMR) pertains to the relationship between the mass of a white dwarf and the mass of its main-sequence progenitor. Hence the IFMR characterises the amount of material a star will return to the interstellar medium during post main-sequence evolution, and so provides information on the mass loss processes which occur towards the end of stellar evolution. The form of this relationship is important in 
the investigation of the previous and ongoing chemical evolution of the Milky Way and galaxies in general. The higher mass end of the IFMR is equally as important in supernovae studies, as predictions about the rate of these explosions are particularly sensitive to the assumed minimum mass of the core-collapse progenitor.

The first attempt to constrain the IFMR was made by Weidemann (1977), and shortly after this Romanishin \& Angel (1980) conducted a purely photographic study of several young open clusters to search for new white dwarf candidates. The expected number of white dwarfs in these clusters were modelled and limits estimated on the upper progenitor mass limits to white dwarf production. Later spectroscopic studies, essential in determining masses and cooling timescales for the white dwarfs, started to produce a more solid estimate of the IFMR in a series of papers by Koester \& Reimers (Koester \& Reimers 1981, Koester \& Reimers 1985, Koester \& Reimers 1993, Koester \& Reimers 1996). This 20 year effort to constrain the IFMR was reviewed in Weidemann (2000).

The most recent work on the IFMR has more than doubled the number of available data points (e.g. Claver et al. 2001, Dobbie et al. 2004, Williams et al. 2004, Ferrario et al. 2005, Dobbie et al. 2006, Casewell et al. 2009). They all found empirically that the IFMR can be represented by a linear function. Dobbie et al. (2006) constrained the IFMR to these values:

$$
M_{\mathrm{I}}=(0.133 \pm 0.015) M_{\mathrm{F}}+(0.289 \pm 0.051) \mathrm{M}_{\odot}
$$


where $M_{\mathrm{I}}$ and $M_{\mathrm{F}}$ are the initial and final masses of the star respectively. This relationship applies to white dwarfs with progenitor masses of $2.7-6 M_{\odot}$, but was later shown to extend down to $1.6 M_{\odot}$ by Kalirai et al. (2008).

\subsubsection{White Dwarf Evolution}

A continued discussion on the post-formation evolution of a white dwarf may seem moot at this point, as the cooling of these dead stars should be relatively simplistic when compared to their previous evolutionary past. However, as was discussed in Section 1.1.4, the spectral types of white dwarfs are varied indeed and so a much more complicated picture emerged slowly over the years, finally arriving at the current theories discussed here.

Stellar evolution predicts the formation of carbon-oxygen core white dwarfs with a helium envelope, and for an additional $80 \%$ of white dwarfs, a hydrogen layer. This accounts for the population of hydrogen atmosphere DA white dwarfs and helium atmosphere DB white dwarfs.

The helium atmosphere white dwarfs are thought to lose the majority of their hydrogen as the result of a late helium flash at the end of the AGB phase (Herwig et al. 1999), resulting in the mixing of the core materials and the helium layer. As the star cools, the carbon and oxygen sink back out of the atmosphere due to high surface gravity, resulting in a hot DO white dwarf. As the star continues to cool, residual hydrogen is thought 
to emerge $\left(T_{\text {eff }} \approx 45,000 \mathrm{~K}\right)$, creating a DA white dwarf with an ultra thin hydrogen atmosphere. This is to account for the existence of the DB gap; a void between $30,000 \mathrm{~K}$ and $45,000 \mathrm{~K}$ where no helium atmosphere DO or DB white dwarfs have been found to exist (MacDonald 1989). At the cool end of the gap, convection dredges up the more massive helium layer cycling the star through a DBA to a DB type atmosphere.

For a hydrogen atmosphere white dwarf evolution appears to be much simpler, with the white dwarf maintaining its pure atmosphere until very low temperatures where convection sets in. Again, this dredges up the inner materials which, until now, have been segregated by the stars large gravitational field. Then, along with the cool DB stars, the increased carbon in the atmosphere accounts for the population of carbon atmosphere DQ white dwarfs. Eventually all white dwarfs will cool to a point where there are no spectral features, becoming DC type stars.

Of course, this is a much simplified version of white dwarf evolution which does not necessarily account for all the existing variations of white dwarf spectral types. Binary interaction also provides another evolutionary channel (CVs), and pre-CV systems may still accrete from the stellar wind of a close secondary star. In fact, the atmospheric evolution of white dwarfs is still a matter of some debate. 


\subsection{White Dwarfs in Binaries}

White dwarfs in binary systems may follow a markedly different evolutionary path compared to their isolated cousins. Observations of such systems allow for the direct comparison of isolated and binary white dwarfs, and can provide evidence for the direct interaction of the components. These observations are critical in the understanding of Common Envelope evolution (CEE, Section 1.2.1), and for the study of angular momentum loss mechanisms such as magnetic stellar wind breaking. Close binaries may also be the progenitors of Cataclysmic Variable (CV) systems.

Near-infrared surveys of white dwarfs enable the detection and study of late stellar and substellar companions, and circumstellar dust disks. A typical white dwarf is $10^{3}-10^{4}$ times fainter than its main sequence progenitor (Figure 1.4), significantly reducing the brightness contrast problem when searching for cool, low mass secondaries and dust. In addition, the spectral energy distributions of most white dwarfs (blue) and their low mass companions (red) are markedly different, facilitating easy separation of the components in broadband photometry and enabling straightforward spectroscopic follow-up (Dobbie et al. 2005). Previous near-infrared photometric surveys of white dwarfs include those by Probst (1983), Zuckerman \& Becklin (1987a), Green et al. (2000a), Farihi et al. (2005), and the analysis of white dwarfs in the Two-Micron All Sky Survey (2MASS; Skrutskie et al. 2006) by Wachter et al. (2003), Wellhouse et al. (2005), Holberg \& Magargal (2005), Tremblay \& Bergeron (2007b) and Hoard et al. (2007). 


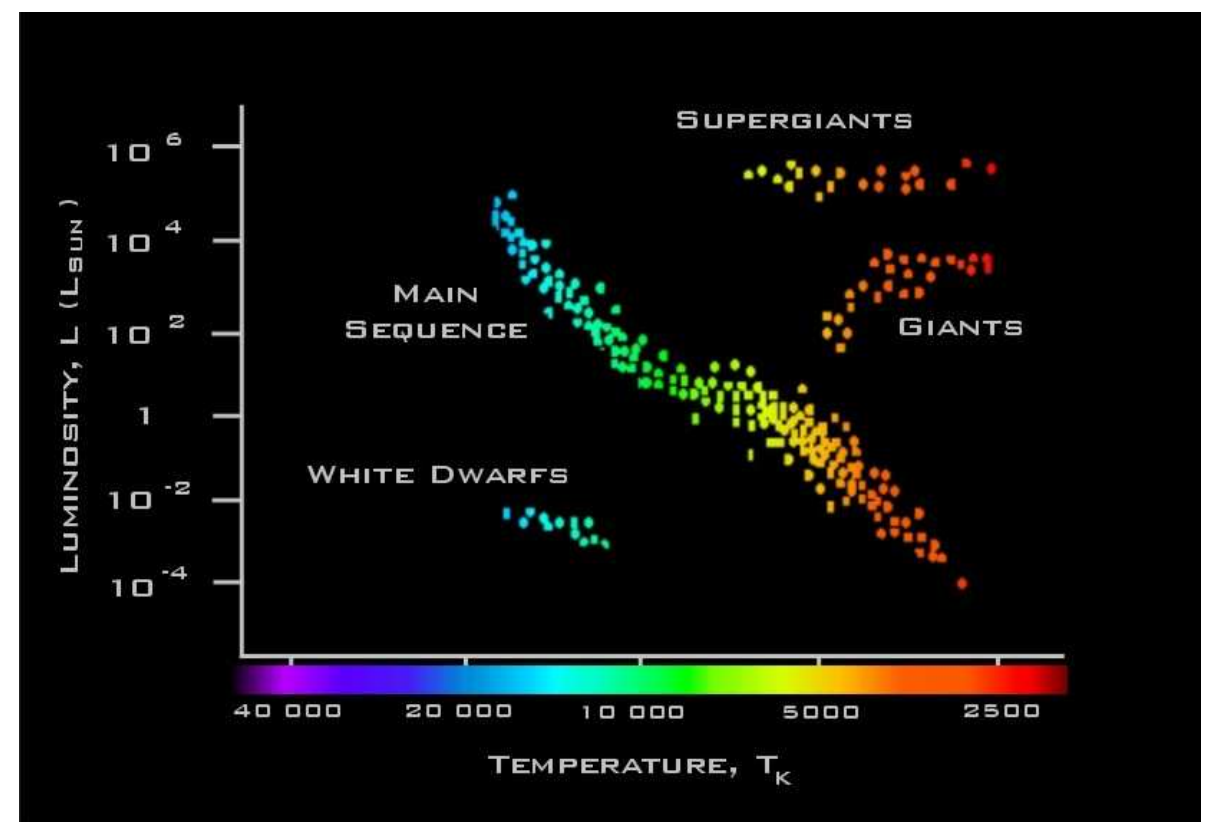

FIGURE 1.4. Hertzsprung-Russel Diagram showing the positions of main sequence stars, white dwarfs, red giants and supergiants. 
Many white dwarfs are known to have low mass $\mathrm{M}$ dwarf companions, and analysis of their population statistics allows the investigation of binary formation and evolution. In particular, consideration of stellar evolution suggests the possible existence of two distinct populations: close systems (Orbital distance $<0.1 \mathrm{AU}$ ) in which the secondary has survived a phase of CEE and which may eventually lead to the formation of a $\mathrm{CV}$, and wide pairs (Orbital separation $>$ few $\mathrm{AU}$ ) where the secondary has migrated outwards in response to mass-loss from the white dwarf's progenitor (Farihi, Hoard \& Wachter 2006a). An object originally orbiting a white dwarf progenitor at $a>5 \mathrm{AU}$ will have its orbit expanded by a maximum factor equal to the ratio of the main-sequence mass to the white dwarf mass $\left(M_{\mathrm{ms}} / M_{\mathrm{wd}}\right)$ (Jeans 1924, Zuckerman \& Becklin 1987b, Burleigh et al. 2002), a value typically around 3. Thus, any companion originally orbiting between 10-100 AU would end up at 30-300 AU following main-sequence evolution. For progenitor systems with a closer initial orbital separation $(<2 \mathrm{AU})$, the secondary is thought to spiral inward during a common envelope phase (Section 1.2.1). Thus, a bimodal separation is expected for the distribution of orbital separations in white dwarf + stellar/substellar binaries (Figure 1.5). As a result no stellar or substellar companions are expected to be found with an orbital distance between 1 to a few AU.

One particularly interesting case are the magnetic white dwarfs in CV systems, which generate large amounts of cyclotron radiation in addition to flux produced from direct accretion. This can be directly detected using $K$-band photometry (e.g. SDSS J121209.31+ 013627.7; Burleigh et al. 2006b, Farihi et al. 2008). Although hundreds of magnetic CVs 
(Polars) are known, no convincing progenitor system has been identified (Liebert et al. 2005) despite exhaustive analysis of the 2MASS dataset for these objects (Wellhouse et al. 2005). 


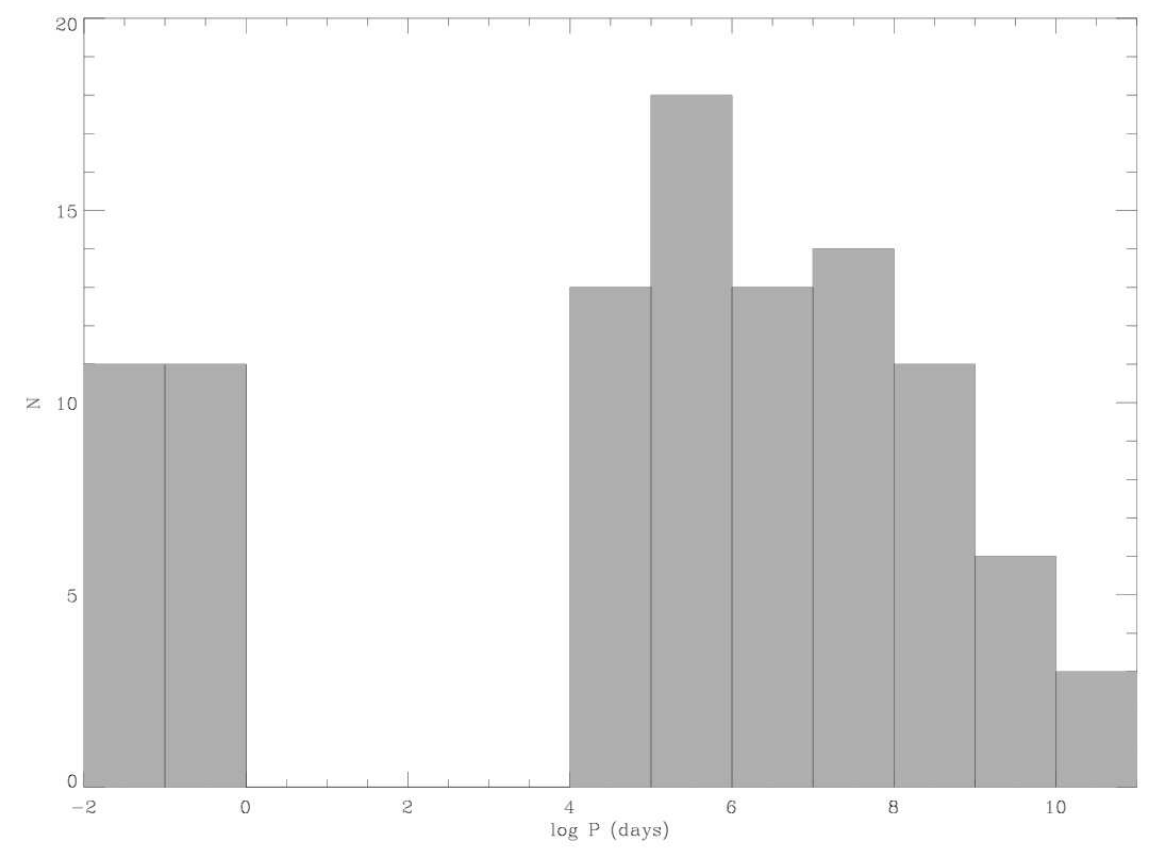

FIGURE 1.5. Simulated binary separation distribution for white dwarfs assuming an initial separation distribution similar to that of G-dwarf binaries. Figure from Farihi (2004). 


\subsubsection{Common Envelope Evolution}

The evolution of initially wide ( $\mathrm{P} \sim$ Years) into close ( $\mathrm{P} \sim$ Hours) binary systems is dependant on the rate at which angular momentum can be extracted from the system. In order to understand the population of close white dwarf binaries, an understanding of the mechanisms which can achieve this outcome is needed. It is generally thought that a significant fraction of the orbital angular momentum is lost during a shortlived common envelope (CE) phase.

When a white dwarf progenitor is on the RGB or AGB, it has a large convective envelope (Section 1.1.5). If a secondary component in the system is close, Roche lobe overflow occurs and the resulting mass transfer causes the primary star to expand. If the primary reaches a point where it can no longer accept the incoming matter at the current rate (The Eddington Limit, King et al. 1997) and the transfer of mass becomes unstable. A CE forms between the two stars, causing the secondary to lose angular momentum and begin spiralling in towards the primary. This decrease in the companion's orbit results in a gain of gravitational energy. If the gravitational energy is large enough to eject the envelope then a closely orbiting binary emerges. If the orbit decreases to the point where the secondary fills its Roche lobe then the stars will merge and the binary will not survive.

However, this survival rate depends on a number of assumptions and input parameters in theoretical models. In particular, a value for the common envelope ejection efficiency parameter, $\alpha$, needs to be assumed (de Kool 1990). This value gives the efficiency at 
which the dumped gravitational energy is being used to eject the CE. Studies have shown this value lies between 0.3 and 1.0. de Kool (1992) show that approximately $20 \%$ and $40 \%$ of secondaries will survive for $\alpha=0.3$ and 1.0 respectively.

So observational studies of close white dwarf binaries can help answer the question "What limits are there to the mass of an object that can survive CE evolution?". Unfortunately, no direct observations of a CE exist as the process is extremely fast (a few to $10^{3}$ years). The lowest mass object currently thought to have survived a $\mathrm{CE}$ is WD $0137-349 \mathrm{~B}$, a brown dwarf with a mass of $53 M_{\mathrm{J}}$ and an orbital period of 116 mins (Maxted et al. 2006, Burleigh et al. 2006a).

CEE is a process which is not particularly well understood. Some theoretical work has been done in answering the question of planetary survival and the general fate of CE systems (e.g. Livio \& Soker 1983, Livio \& Soker 1984, Nelemans \& Tauris 1998, Villaver \& Livio 2007), however these theories rest on numerous contestable assumptions. Predictions have been made of the masses of planets that will survive a CE phase. For example, Nelemans \& Tauris (1998) calculated the predicted final outcome of CEE for different planetary masses and initial periods for a progenitor star of $1.0 M_{\odot}$ (Figure 1.6). It is important that these theories be tested through observations of systems of low secondary mass and a variety of periods.

Also, if the resulting binary is close enough that it will reach a semi-detached phase through the loss of angular momentum within the age of the Galaxy, then it can be considered to be a pre-CV. If the secondary in the system is a main-sequence star then 


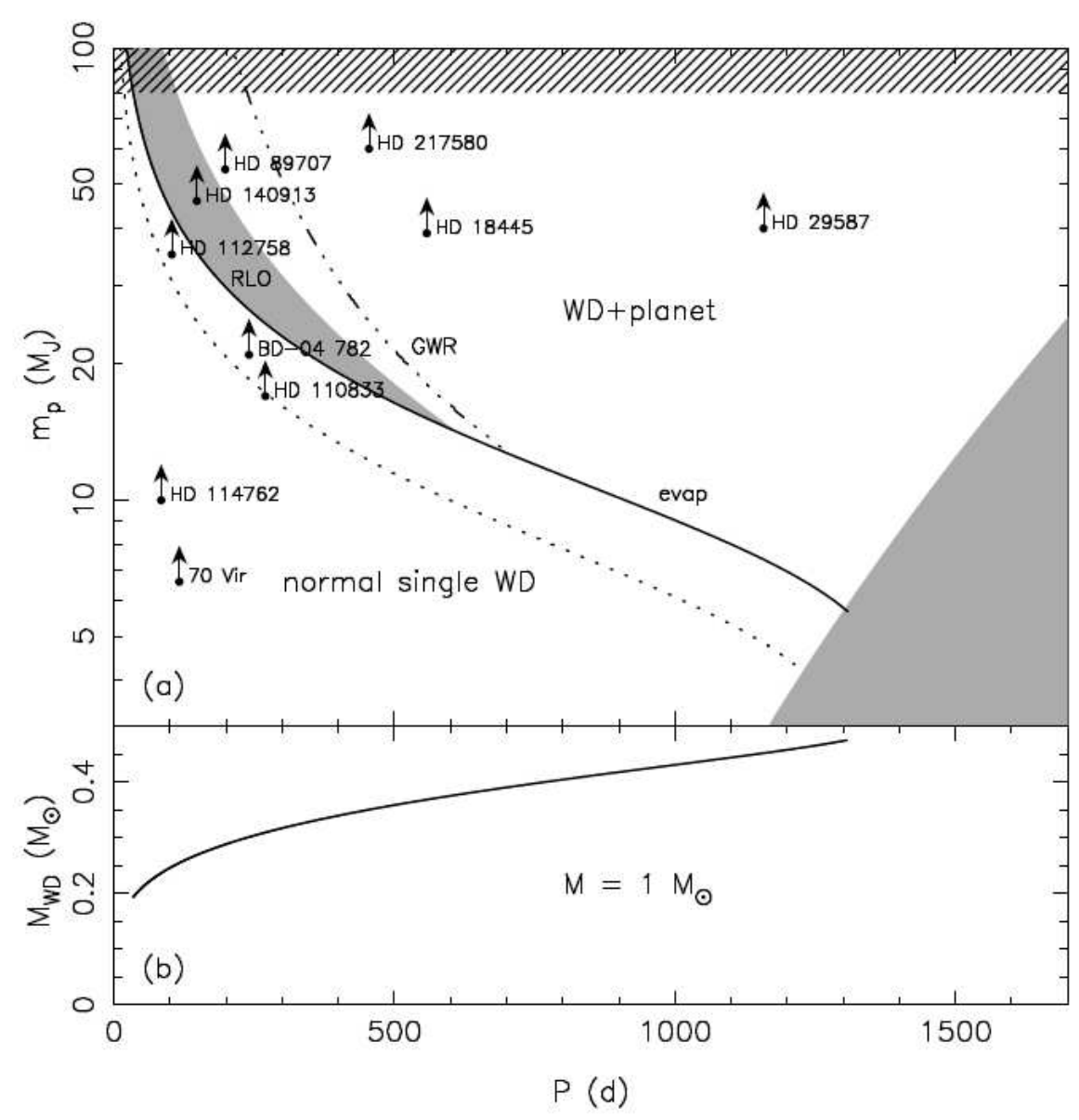

FIgURE 1.6. (a) Predicted final outcome of CEE for different planetary masses and initial periods around a $1.0 M_{\odot}$ star. The solid line represents the critical mass below which the planet will evaporate during the spiral-in phase. The shaded region to the right shows where a planet would be too far away to be engulfed by an envelope whilst the star is on the RGB. (b) Final mass of the white dwarf in the case where all the envelope is expelled. Reproduced from Nelemans \& Tauris (1998) 
this also must occur before this star evolves off the main-sequence.

\subsubsection{Brown Dwarfs}

A brown dwarf is often described as a failed star. That is to say it never gained enough mass to begin the sustained nuclear fusion of hydrogen and thus prevent gravitational collapse. The core temperature of a brown dwarf progenitor reaches a point where it will begin a short phase of deuterium burning during its early life, maintaining hydrostatic equilibrium. Once this supply ultimately runs dry, the brown dwarf continues to collapse to a point where electron degeneracy pressure (Section 1.1.3) becomes the dominant force against further gravitational collapse, and the brown dwarf's radius stabilises.

As a result, brown dwarfs are intrinsically less bright and much cooler than main-sequence stars. This gives a brown dwarf a markedly different atmospheric spectrum that cannot be classified by the existing Harvard scheme.

Thus far, two new spectral types (Kirkpatrick 1998, Kirkpatrick et al. 1999) have been assigned to describe the atmospheres of observed brown dwarfs; (1) L-dwarfs have a temperature range between $\sim 1400-2500 \mathrm{~K}$ (Kirkpatrick 2005), and are spectroscopically characterised by the depletion of titanium oxide and vanadium oxide absorption lines, and the onset of metal hydride (e.g. iron hydride, chromium hydride), neutral alkali (e.g. lithium, sodium, potassium) and carbon monoxide absorption in the optical and near-infrared. (2) T-dwarfs have a temperature range between $\sim 750-1400 \mathrm{~K}$ and are 
spectroscopically characterised by strong water and hydrogen absorption, and the onset of methane absorption in the near-infrared.

\subsubsection{Brown Dwarf Atmospheres}

Brown dwarf atmospheres are notoriously difficult to model due to their cool atmospheres $\left(T_{\text {eff }}<3500 \mathrm{~K}\right)$ which contain complex molecules (e.g. $\left.\mathrm{TiO}, \mathrm{H}_{2} \mathrm{O}, \mathrm{CH}_{4}\right)$. The presence of such molecules leads to uncertainties in predicting opacities in the atmospheres, and in the coolest brown dwarfs these molecules condense into grains causing further issues.

In 1997 Chabrier \& Baraffe used the models of Allard et al. (1997) to produce models of the evolution and structure of low mass, dense objects. These became the NEXTGEN models (Baraffe et al. 1998), which apply to objects between $0.01 M_{\odot}$ and $1.0 M_{\odot}$, and $T_{\text {eff }}>2200 \mathrm{~K}$. For cooler objects the DUSTY models (Chabrier et al. 2000) are used, which take into account the formation of dust in the brown dwarf's atmosphere and the resultant absorption and scattering. These cover the mass and effective temperature ranges $0.01<M<0.1 M_{\odot}$ and $900<T_{\text {eff }}<2800 \mathrm{~K}$ respectively, for objects with ages between $10^{8}$ and $10^{10}$ years. However, the DUSTY models tend to be ineffective for $T_{\text {eff }}<1400 \mathrm{~K}$ where the dust clouds become less important as they sink below the photosphere. This marks the beginning of the T dwarfs, whose spectra are more successfully produced by the COND models (Baraffe et al. 2003). In these models the heavy elements which form dust (e.g. Fe, Ti and V) are removed from the atmosphere. 


\subsubsection{White Dwarfs with Sub-Stellar Companions}

The mass-luminosity relation for brown dwarfs is strongly dependant on age, a factor which cannot be determined from the luminosity of an isolated brown dwarf. The key to understanding these objects is to be able to determine the mass and age of the brown dwarf independently of atmospheric models. So brown dwarfs in binary systems with white dwarfs are excellent candidates for these so called benchmark brown dwarfs (Farihi et al. 2005, Pinfield et al. 2006). This is because the white dwarf's age can be determined relatively accurately, inferring an age for the brown dwarf secondary. A benchmark brown dwarf + white dwarf binary can therefore be used to help constrain substellar evolutionary models at intermediate to older ages $(>1 \mathrm{Gyr})$ such as those expected for most white dwarfs.

Substellar companions to white dwarfs additionally allow the investigation of the known deficit of brown dwarf companions to main sequence stars $(<10 \mathrm{AU}: 0.5 \%$; Marcy $\&$ Butler 2000). The closest brown dwarf + white dwarf binaries might also represent either another channel for $\mathrm{CV}$ evolution or the end state of $\mathrm{CV}$ evolution, in which the secondary has become highly evolved through mass transfer. In close detached binaries, the brown dwarf is expected to be irradiated by the white dwarf's high UV flux, possibly leading to substantial temperature differences between the "day" and "night" hemispheres. Such systems can provide laboratories for testing models of irradiated "hot Jupiter" atmospheres (e.g. HD 189733b; Knutson et al. 2007). 
However, detached brown dwarf companions to white dwarfs are rare $(<0.5 \%$ for Ldwarfs; Farihi et al. 2005). Prior to this thesis, radial velocity and proper motion surveys, and searches for near-infrared excesses have so far found only three confirmed examples: GD 165 (DA+L4, Becklin \& Zuckerman 1988), GD 1400 (DA+L6 - 7; Farihi \& Christopher 2004, Dobbie et al. 2005), and WD 0137 - 349 (DA+L8; Maxted et al. 2006, Burleigh et al. 2006a). GD 165 is a wide orbit binary with a projected separation of $120 \mathrm{AU}$, whereas GD 1400 and WD 0137-349 are in much closer orbits with periods of 10 hours (Burleigh et al. 2009, in prep) and 116 mins respectively. To this date no WD $+\mathrm{dT}$ type binaries have been identified.

\subsection{White Dwarfs with Debris Disks}

Infra-red observations have recently revealed the presence of metal-rich debris dust disks around a small number of white dwarfs. Indeed, the large near-infrared excess in G 29-38 was first identified twenty years ago by Zuckerman \& Becklin (1987a). Mid-infrared photometry showed a more pronounced excess at $\sim 10 \mu \mathrm{m}$ than could be fitted with a blackbody of $T_{\text {eff }} \sim 10^{3} \mathrm{~K}$. The favoured explanation for the origin of this material is the tidal disruption of an asteroid that has wandered into the Roche radius of the white dwarf (Jura 2003).

Zuckerman et al. (2003) did a survey of DA white dwarfs and found that, for those stars not in a detectable binary, $\sim 25 \%$ had an excess of metals in their atmospheres. The metals present should sink from the photospheres on a timescale of days, so the presence 
of close, orbiting dust disks provides a reservoir for on-going low level accretion. A further 11 white dwarfs have been found to show evidence of a similar near-infrared excess to that of G29-38 (Becklin et al. 2005, Kilic et al. 2005, Kilic et al. 2006, von Hippel et al. 2007, Jura et al. 2007, Farihi et al. 2008, Jura et al. 2009, Farihi et al. 2009). The majority of these were found around cool metal rich DAZ white dwarfs, with effective temperatures $T_{\text {eff }}<15,000 \mathrm{~K}$. Kilic et al. (2006) suggested that for white dwarfs with $T_{\text {eff }}>16,000-20,000 \mathrm{~K}$ any debris disk would have been sublimated. One notable exception is the central star of the Helix nebula. This hot, extremely young white dwarf has a mid-infrared excess emission identified with Spitzer by Su et al. (2007), which might be explained by the presence of a ring of colliding cometary material at an orbital distance of $\approx 30 \mathrm{AU}$.

More recently, evidence has been found for gas disks around 3 moderately hot white dwarfs (Gänsicke et al. 2006, Gänsicke et al. 2007, Gänsicke et al. 2008). These all exhibit double-peaked emission lines in the $i$-band Ca II triplet, with a morphology which implies a rotating circumstellar gas disk relatively close to the white dwarf. One of these stars has a spectral classification of DBZ (SDSS0845+2257) implying that not all disks need be found around hydrogen atmosphere DA white dwarfs. Brinkworth et al. (2009) went on to find evidence with the Spitzer Space Telescope of a dusty extension to one of these stars (SDSS1228+1040).

Debris dust and gas disks around white dwarfs may have a variety of origins linked to the post-main sequence evolution of both the central stars and surrounding planetary 
systems. Large infrared surveys will help establish how common they are. 


\section{Chapter 2}

\section{White Dwarfs in UKIDSS}

\subsection{Overview}

This chapter presents a search for spectroscopically identified white dwarfs present in the UKIDSS Large Area Survey Data Release 5. I search within a subgroup of hydrogen and helium atmosphere white dwarfs for near-infrared excesses indicative of the presence of a low mass companion or debris disk. 23 new candidate photometric white dwarf + very low mass companion binaries are identified. I then calculate an expected mass for a number of the putative companions and find that 8 are within the mass range of brown dwarfs.

\subsection{The UKIDSS Survey}

The UKIRT Infra-red Deep Sky Survey (UKIDSS) is the near-infrared (NIR) counterpart to the optical Sloan Digital Sky Survey (SDSS: York et al. 2000) and is the successor to 
the previous NIR 2 Micron All Sky Survey (2MASS: Skrutskie et al. 2006). UKIDSS began in May 2005 and aims to cover $7500 \mathrm{deg}^{2}$ of the Northern Sky in $Y J H K$ down to $K=18.4$ mags, 3 magnitudes deeper than 2MASS. UKIDSS actually consists of a set of 5 complimentary surveys; the Large Area Survey (LAS), the Galactic Plane Survey (GPS), the Galactic Clusters Survey (GCS), the Deep Extragalactic Survey (DXS) and the Ultra-deep Extragalactic Survey (UDS). Following a release of science verification data and an Early data release (Dye et al. 2006), UKIDSS has had 5 data releases (DR1: Warren et al. 2007b, DR2: Warren et al. 2007a, DR3, DR4 and DR5) in July 2006, March 2007, December 2007, July 2008 and April 2009. Each subsequent data release is inclusive of all data from the previous releases. The survey of most importance to this work is the Large Area Survey.

\subsubsection{The Large Area Survey}

The LAS aims to cover $4000 \mathrm{deg}^{2}$ upon it's 7 year completion down to $5 \sigma$ limiting magnitudes of $Y=20.5, J=20.0, H=18.8$ and $K=18.4$ mags (Vega). The target $4000 \mathrm{deg}^{2}$ is a subsection of the Sloan survey with the selected fields shown in Figure 2.1. It will cover: (1) a part of the SDSS equatorial block, (2) a part of the SDSS northern block, and (3) a section of the southern equatorial stripe.

UKIDSS uses the Wide-Field Camera (WFCAM: Casali et al. 2007) on the United Kingdom Infra-red Telescope (UKIRT). LAS observations are carried out in the $Y, J, H$ and $K$ filters with the goal of reaching a uniform depth in all fields. The minimum schedu- 


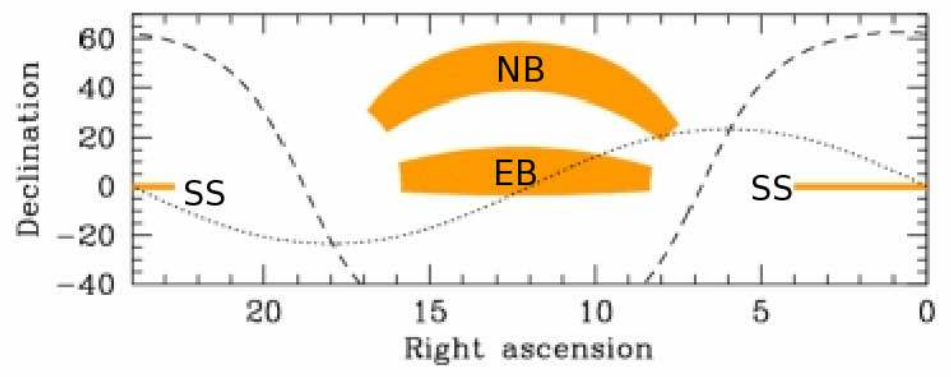

FIGURE 2.1. Planned final LAS sky coverage. The equatorial block (EB) corresponds to SDSS stripes 9 to 16, the northern block (NB) corresponds to SDSS stripes 26 to 33, and the southern stripe (SS) is part of SDSS stripe 82. The dashed line marks the Galactic plane, and the dotted line marks the ecliptic. 
lable blocks (MSBs) for the LAS use a pair of filters; either $Y J$ or $H K$. If an area has been covered by one pair an effort is made to follow up with the remaining pair within one month. However, conditions do not always allow for this and observations depend on a number of constraints (Dye et al. 2006). Hence, before completion a small number of observations in each data release will only have measurements in either the $Y J$ or $H K$ bands only. It is also entirely possible that any single measurement will not pass data quality tests and be omitted from the database. Therefore, a number of targets may have a selection of $Y, J, H$ or $K$ magnitudes, but not all four.

\subsection{Candidate Selection}

\subsubsection{Identifying White Dwarfs}

The UKIDSS LAS DR5 catalogue was cross correlated with two catalogues of white dwarfs: the on-line August 2006 version of the McCook \& Sion catalogue of spectroscopically identified white dwarfs (McCook \& Sion 1999) which contains 5557 stars, and the Eisenstein et al. (2006) (hereafter EIS06) catalogue of 9316 spectroscopically confirmed white dwarfs from the SDSS DR4. The McCook \& Sion catalogue contains the brightest white dwarfs known, whilst the white dwarfs identified in the SDSS are constrained by an upper brightness limit $\left(g^{\prime} \approx 15\right)$ and the lower sensitivity limit $\left(g^{\prime} \approx 21\right)$ of that survey. The majority of the SDSS white dwarfs are too faint to have been detected by the 2MASS survey, therefore UKIDSS allows the investigation of the NIR properties of a large number of new white dwarfs discovered in the SDSS, as well as significantly 
reducing the measurement errors on the NIR photometry of the brighter white dwarfs detected in 2MASS.

Many of the relatively bright white dwarfs have large and unmeasured proper motions, meaning their astrometry is often unreliable. For the McCook \& Sion white dwarfs, a set of improved coordinates supplied by Jay Holberg (private communication) were utilised in the cross correlation with UKIDSS DR5. The SDSS stars, being fainter and therefore more distant have more reliable astrometry. The cross correlation was performed using the on-line "CrossID" tool at the WFCAM science archive. The results were constrained to stars brighter than the $5 \sigma$ limiting magnitudes (Section 2.2.1) using a pairing radius of $5^{\prime \prime}$ taking only the nearest infrared source to each white dwarf's coordinates as the primary result. In general, the UKIDSS and white dwarf coordinates agreed within $5^{\prime \prime}$ and the SDSS images revealed the same object as in the corresponding UKIDSS images. In several cases the target star was already faint in the SDSS and did not become significantly brighter in the NIR as to be detected by UKIDSS (i.e. the white dwarf was not detected in the NIR and there was no companion/disk present). The images of each cross matched object in both databases were inspected by eye to ensure the correct object had been identified. Eleven of the sample were rejected as being the wrong source in the UKIDSS when compared to the SDSS image.

The cross correlation produced 1161 matches with the SDSS DR4, and 489 with the McCook \& Sion white dwarf catalogues. Only 106 of the McCook \& Sion white dwarfs were not found in the SDSS. The summary of spectral types for the white dwarfs are 
listed in Table 2.1.

\subsubsection{The Near-infrared Two Colour Diagram}

In order to make an immediate and rapid search for white dwarfs with a NIR excess, indicative of a low mass companion or dust disk, the UKIDSS DR5 white dwarfs were plotted on the $(J-H, H-K)$ two-colour diagrams shown in Figure 2.2. This method was first applied by Wachter et al. (2003) in their analysis of white dwarfs detected by 2MASS. Then, based on Wachter et al.'s work and theoretical colour simulations, Wellhouse et al. (2005) further developed the technique by splitting the two-colour diagram into four distinct regions; (I) single white dwarfs, (II) white dwarfs with late-type main-sequence companions, (III) white dwarfs with lower mass, possible brown dwarf companions and (IV) white dwarfs contaminated with circumstellar material. Wellhouse et al.'s regions have been plotted in the two-colour diagrams in Figure 2.2.

Hoard et al. (2007) argue this colour selection method allows a simple, fast and efficient means to exploit large, homogeneous survey data to provide an initial list of candidate stars with an interesting NIR excess. Hoard et al. (2007) confirmed $80 \%$ of the white dwarf + main-sequence binaries reported by Wachter et al. (2003) with a false positive rate (i.e. stars rejected as misidentification by Wachter et al. 2003) of 20\%. However, they failed to identify any new candidate brown dwarf companions or dust disks. This is probably in part due to the excess emission from additional unresolved sources being small compared to the errors on the 2MASS data. However, it could also be due to some 
Table 2.1. Number of each white dwarf spectral type detected in the cross correlation of UKIDSS LAS DR5

\begin{tabular}{cc|cc|cc}
\hline Spectral Type & No. & Spectral Type & No. & Spectral Type & No. \\
\hline \hline DA & 757 & DO & 4 & DB_O & 1 \\
DA+M & 272 & DB_A & 4 & DAO & 1 \\
DC & 40 & PG1159 & 3 & DZB & 1 \\
DB & 25 & DZA & 3 & DO+M & 1 \\
DQ & 18 & DBH & 3 & DAZ & 1 \\
DZ & 13 & DBZ & 3 & DZ+M & 1 \\
DAH & 14 & DAB & 2 & DBA+M & 1 \\
DBA & 11 & DH & 2 & DAH+DA & 1 \\
WD+M & 8 & DAOB & 2 & DAH+M & 1 \\
DB+M + DA & 5 & DA_B & 1 & & \\
DC+M + D & 5 & DZA+M & 1 & & \\
\hline
\end{tabular}



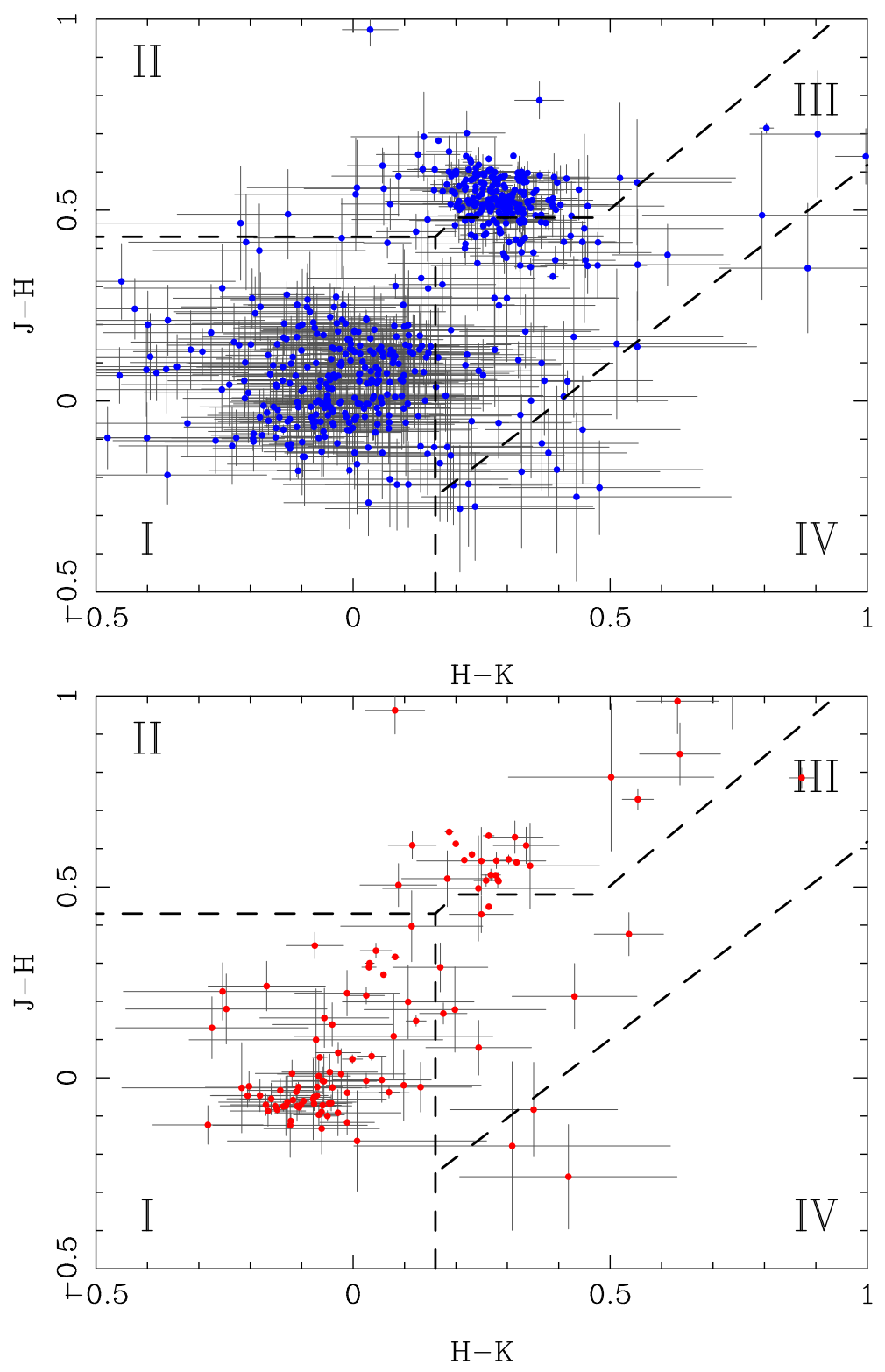

FIGURE 2.2. $J-H$ vs $H-K$ colour-colour diagram for the SDSS white dwarfs (top) and the McCook \& Sion white dwarfs (bottom) with complete $J H K$ photometry present in the UKIDSS archive. Nb. the overlap between the 2 catalogues has not been accounted for in these diagrams. The four regions delineate (I) single white dwarfs, (II) white dwarfs with late-type main-sequence companions, (III) white dwarfs with lower mass, possible brown dwarf companions and (IV) white dwarfs contaminated with circumstellar material. 
white dwarfs with companion brown dwarfs or dust disks occupying regions (I) and (II) of the two-colour diagram, making them indistinguishable from single white dwarfs or the large population of white dwarfs with main sequence $\mathrm{K}$ and $\mathrm{M}$ dwarf companions.

To investigate this further, white dwarfs with known brown dwarf companions and dust disks have been plotted in a NIR two-colour diagram in Figure 2.3 using photometry from 2MASS and other sources in literature (See Tables 2.2 and 2.3). Clearly only seven of the fourteen white dwarfs plotted lie within regions where further investigation would have been solicited using Wellhouse et al.'s (2003) regions. Indeed only a single dust disk (GD56) and a single white dwarf + brown dwarf low accretion rate CV (SDSS121209.31+013627.7, Burleigh et al. 2006b) lie within the regions expected of such systems. In this case it can be concluded that the colour selection method is likely to easily distinguish $\sim 50 \%$ of such systems. However, more examples would be required to accurately constrain such statistics.

It should also be noted that this method can only be used to analyse white dwarfs which have complete $J H K$ photometry. In Section 2.2.1 it was noted that some stars will only have either $Y J$ or $H K$ photometry at this time in UKIDSS and so can not be included in this analysis. The sample of white dwarfs selected here has $47 \%$ of stars which are missing one of the needed $J, H$ or $K$-band magnitudes (Table 2.4).

Other limitations of the colour selection method are discussed by Tremblay \& Bergeron (2007), who critically analysed the 2MASS photometric data for white dwarfs and the analyses of Wachter et al. (2003) and Wellhouse et al. (2005). They concluded that the 


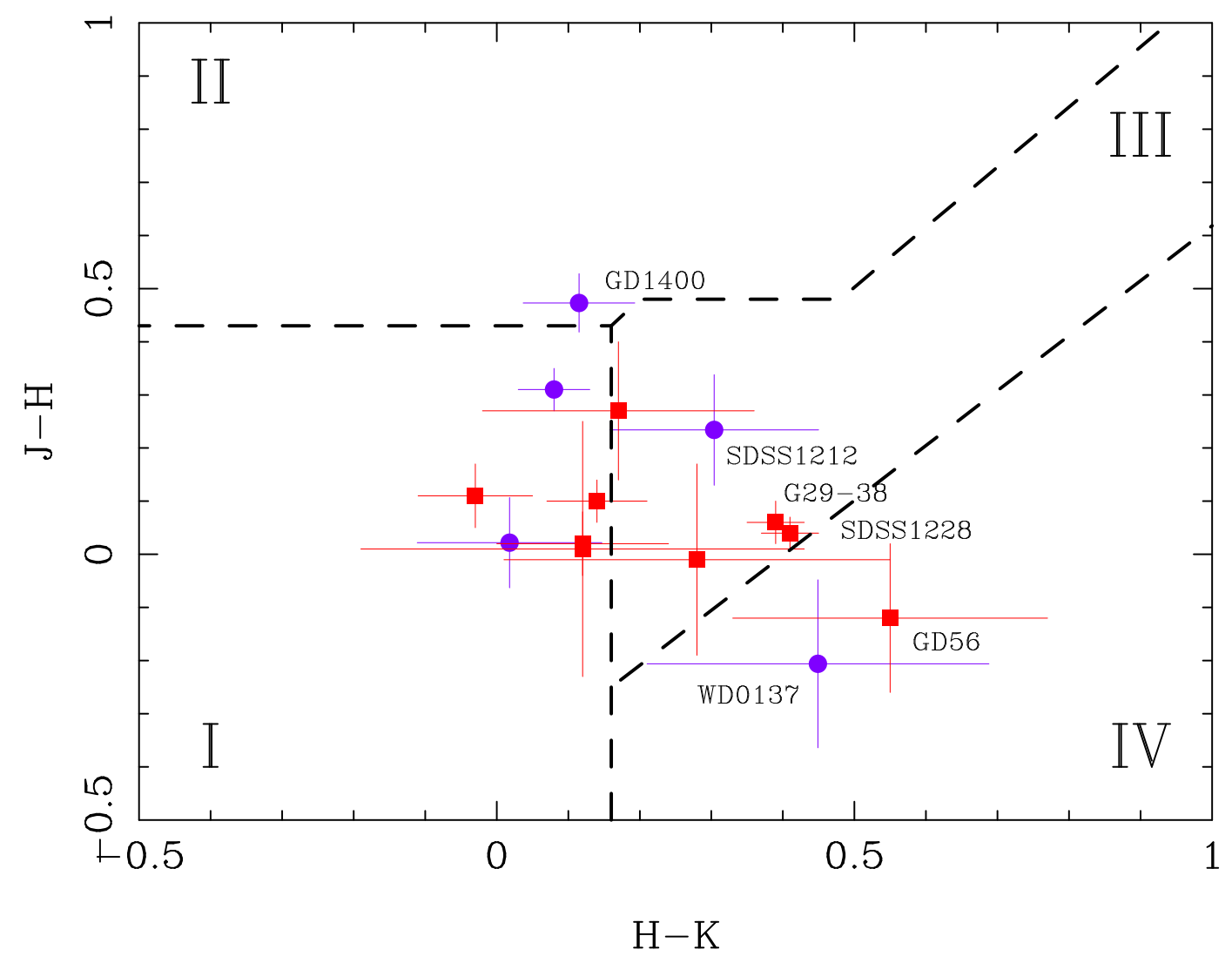

FIGURE 2.3. Near-infrared colour-colour diagram of white dwarfs with confirmed low-mass companions (purple) and circumstellar disks (red). 
Table 2.2. Near infra-red magnitudes of known white dwarfs with unresolved brown dwarf companions

\begin{tabular}{lcccc}
\hline Name & $J$ & $H$ & $K$ & Source \\
\hline \hline WD 0137 - 349 & $15.681 \pm 0.055$ & $15.887 \pm 0.148$ & $15.438 \pm 0.188$ & 2MASS $^{1}$ \\
GD 1400 & $14.923 \pm 0.032$ & $14.450 \pm 0.045$ & $14.335 \pm 0.064$ & 2MASS $^{1}$ \\
SDSS J121209.31 +013627.7 & $17.91 \pm 0.05$ & $17.676 \pm 0.091$ & $17.372 \pm 0.114$ & Farihi et al. (2008) $^{1}$ \\
PG 1234 + 482 & $14.977 \pm 0.043$ & $14.955 \pm 0.073$ & $14.937 \pm 0.106$ & 2MASS $^{1}$ \\
PHL 5038 & $16.75 \pm 0.02$ & $16.44 \pm 0.03$ & $16.36 \pm 0.04$ & Steele et a. (2009) \\
\hline
\end{tabular}

${ }^{1}$ Skrutskie et al. (2006)

${ }^{2}$ Partially resolved in UKIDSS

Table 2.3. Near infra-red magnitudes of known white dwarfs with circumstellar debris disks

\begin{tabular}{|c|c|c|c|c|}
\hline Name & $J$ & $H$ & $\bar{K}$ & Source \\
\hline$\overline{\mathrm{G} 29-38}$ & 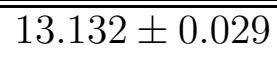 & $1313.075 \pm 0.022$ & 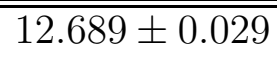 & $2 \mathrm{MASS}^{1}$ \\
\hline GD 362 & $16.09 \pm 0.03$ & $15.99 \pm 0.03$ & $15.85 \pm 0.06$ & Becklin et al. (2003) \\
\hline GD 56 & $15.870 \pm 0.061$ & $15.991 \pm 0.129$ & $15.440 \pm 0.175$ & $2 \mathrm{MASS}^{1}$ \\
\hline WD $2115-560$ & $14.110 \pm 0.029$ & $13.996 \pm 0.055$ & $14.022 \pm 0.061$ & $2 \mathrm{MASS}^{1}$ \\
\hline GD 40 & $15.885 \pm 0.072$ & $15.897 \pm 0.161$ & $15.621 \pm 0.214$ & $2 \mathrm{MASS}^{1}$ \\
\hline PG $1015+161$ & $16.131 \pm 0.085$ & $16.120 \pm 0.222$ & $16.003 \pm 0.216$ & $2 \mathrm{MASS}^{1}$ \\
\hline GD 133 & $14.752 \pm 0.039$ & $14.730 \pm 0.051$ & $14.611 \pm 0.105$ & $2 \mathrm{MASS}^{1}$ \\
\hline GD 16 & $15.799 \pm 0.076$ & $15.528 \pm 0.101$ & $15.358 \pm 0.163$ & $2 \mathrm{MASS}^{1}$ \\
\hline SDSS J122859.93 + 104032.9 & $16.897 \pm 0.016$ & $16.856 \pm 0.023$ & $16.442 \pm 0.038$ & UKIDSS DR5 \\
\hline
\end{tabular}

\footnotetext{
${ }^{1}$ Skrutskie et al. (2006)
} 
method is appropriate but should be interpreted with caution near the detection threshold where larger errors are to be expected. They demonstrated that there exists a number of single stars with large photometric uncertainties that contaminate regions (III) and (IV) of the colour-colour diagram. Indeed, It can be seen in Figure 2.2 that there are a number of stars with errors bars that extend from regions (III) and (IV) back into regions (I) or (II).

Tremblay \& Bergeron (2007) demonstrate that a more thorough technique is to compare observed NIR photometric fluxes with those predicted by model atmospheres (as also adopted by Holberg \& Magargal 2005). This method first requires accurate effective temperatures and surface gravities, which are determined from high S/N optical spectroscopy. The model spectral energy distribution (SED) is then normalised to the observed $V$ or $g^{\prime}$ magnitude of the white dwarf. Predicted photometry can then be calculated by folding the synthetic SED through the appropriate filter transmission profiles and a direct comparison made. However, this technique is limited to those stars with known effective temperatures and surface gravities. Many of the McCook \& Sion white dwarf do have accurately determined temperatures and gravities. The SDSS DR4 white dwarfs do have published atmospheric parameters (EIS06), however these are determined in an automated fashion and users of the catalogue are warned to be cautious when adopting these values.

The colour-colour diagram of the UKIDSS white dwarfs (Figure 2.2) clearly separates out those stars with optical companions into the bottom of region (II). The majority of 
Table 2.4. The combinations of UKIDSS data available for the 1161 SDSS DR4 white dwarfs.

\begin{tabular}{cc}
\hline Bands Available & No \\
\hline \hline$Y J H K$ & 561 \\
$Y J H$ & 330 \\
$Y H K$ & 27 \\
$Y J K$ & 26 \\
$J H K$ & 53 \\
$Y H$ & 19 \\
$J K$ & 3 \\
$J H$ & 31 \\
$J K$ & 2 \\
$H K$ & 109 \\
\hline
\end{tabular}


stars are in region (I) potentially indicating that these are single white dwarfs. There are then a small number of stars in regions (III) and (IV) which would warrant further investigation. It should be noted that a number of the clear outliers (e.g. the McCook \& Sion object at approximately $H-K=0.08, J-H=0.97)$ are classified in the UKIDSS database as merged sources. This could indicate contamination, perhaps by an background galaxy or star. It can also indicate that a companion is becoming resolved in the NIR, as is most likely the case for e.g. SDSS J020538.12+005835.3 which clearly has a companion in the optical (Figure 2.4) and UKIDSS classes as a merged source, as can be seen from the science images (Figure 2.5). The UKIDSS mergedClass statistic ( -1 for a point source, +1 for an extended source) was checked for each candidate object to assess these possibilities.

It is clear there are significant advantages to using the modelling technique over the colour-colour diagram; a direct comparison can be made between observed and expected photometry determined from UKIDSS filter transmission profiles, and those objects with photometry in only one pair of filters can be analysed. Therefore a variation of this method has been adopted for this work.

\subsubsection{White Dwarf Modelling}

In order to efficiently process the cross-matched catalogue of white dwarfs it was decided to model each white dwarf independently and then inspect each for evidence of a NIR excess. It was also decided that only those DA and DB white dwarfs with both $H$ and $K$ 


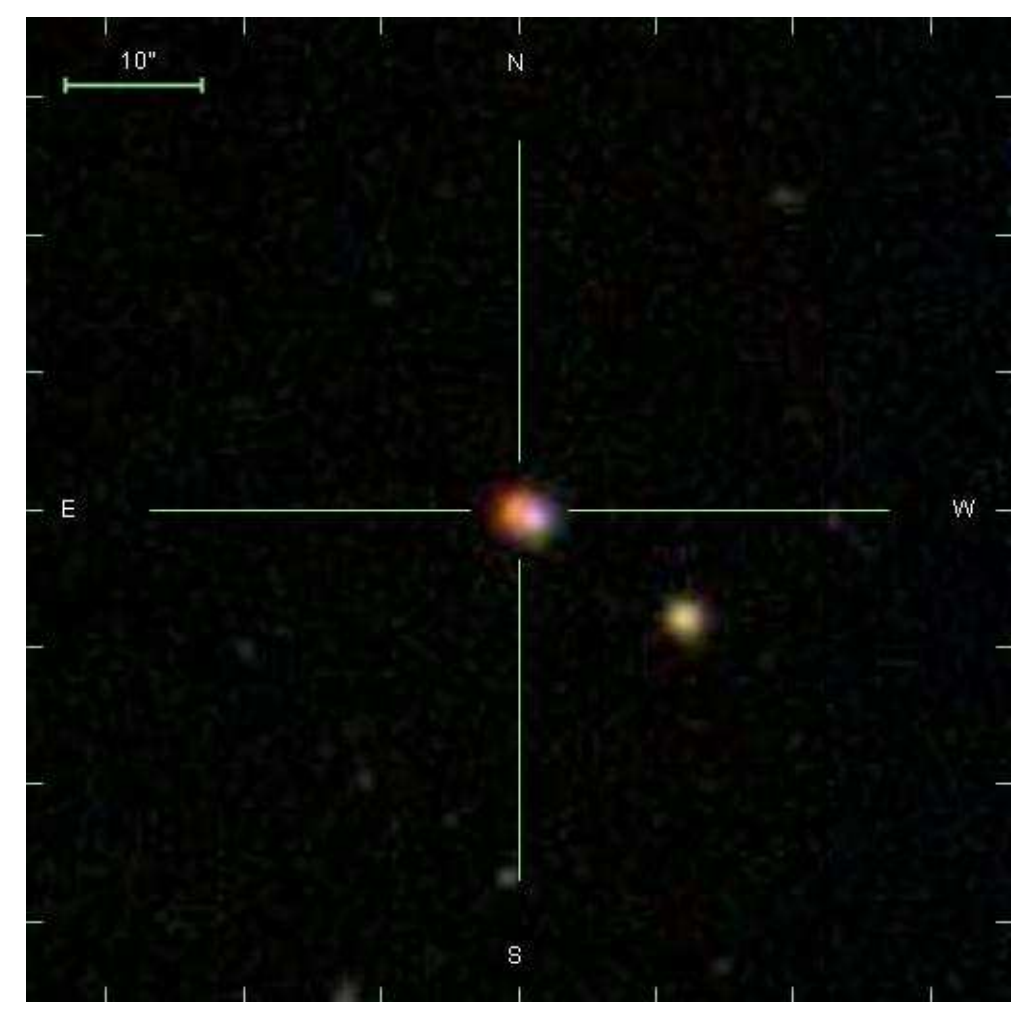

FIGURE 2.4. SDSS finder image of SDSS J020538.12+005835.3 clearly showing both system components.

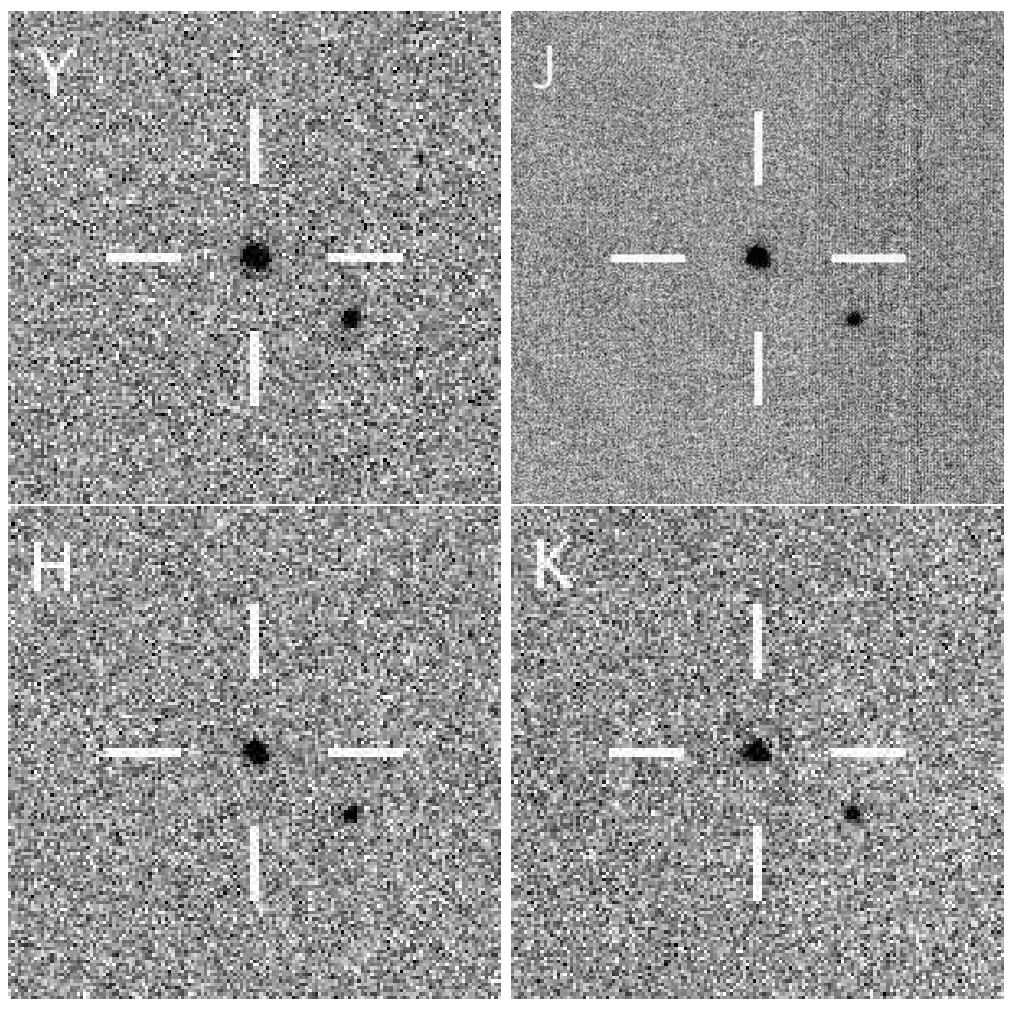

FIGURE 2.5. UKIDSS $Y J H K$ images of SDSS J020538.12+005835.3. 
band photometry were to be modelled, as it is unlikely that the existence of a substellar companion could be inferred from a $Y J$-band excess alone, and both $H$ and $K$-band photometry will allow companion and disk systems to be more easily distinguished. To that end, a program was written to interpolate the Fontaine cooling models for DA and DB white dwarfs. This grid is built from a local thermodynamic equilibrium (LTE) model atmosphere code (Bergeron et al. 1992), which incorporates convective energy transport and hydrogen molecular opacity, up to an effective temperature $\left(T_{\text {eff }}=20,000 \mathrm{~K}\right)$ where non-LTE effects are still negligible and the stellar atmosphere is completely radiative. For the pure hydrogen white dwarfs with $T_{\text {eff }}>20,000 \mathrm{~K}$, the grid then switches to using the plane-parallel, hydrostatic, non-LTE atmosphere and spectral synthesis codes TLUSTY (v200; Hubeny 1988; Hubeny \& Lanz 1995) and SYNSPEC (v49; Hubeny \& Lanz, ftp:/tlusty.gsfc.nasa.gov/synsplib/synspec). For the pure helium white dwarfs the models are similar but with a fractional hydrogen mass of $\mathrm{q}_{\mathrm{H}}=10^{-10}$. The Bergeron model grid extends from $1500<T_{\text {eff }}<100,000 \mathrm{~K}$ and $7.0<\log g<9.0$ for DA white dwarfs, and $3500<T_{\text {eff }}<30,000 \mathrm{~K}$ and $7.0<\log g<9.0$ for DB white dwarfs. The DA model grid was extrapolated to $6.5<\log g<9.5$ to include the lower and higher mass white dwarfs, but to preclude any possible sub-dwarf contamination of the sample.

This procedure predicted absolute magnitudes for the SDSS $i^{\prime}$ and the 2MASS $J H K_{\mathrm{S}}$ filters, as well as an estimated distance to the white dwarf and cooling age of the system. The 2MASS magnitudes were converted to the corresponding UKIDSS filters using the colour transformations of Carpenter (2001). Out of the 1037 white dwarfs classified by EIS06 and McCook \& Sion as DA white dwarfs, 639 had both $H$ and $K$-band photometry 
and fell within the extended DA model grid. Only 10 DB white dwarfs had both $H$ and $K$-band photometry and fell within the DB model grid.

Candidate stars were identified as those with no previously identified companion/disk, whilst showing a $>3 \sigma$ excess in either both the UKIDSS $H$ and $K$-bands, or the $K$-band only, when compared to the model predicted values. 235 such excesses were identified, with 206 of these accounted for as previously identified DA+dM or DB $+\mathrm{dM}$ type binaries from a red excess in SDSS (or other optical data). This left 29 candidate white dwarfs with an apparent NIR excess. The UKIDSS photometry for these is given in Table 2.5 with additional parameters given in Table 2.6.

\subsubsection{Identifying Sub-Stellar Companions}

For each candidate white dwarf + brown dwarf binary the SDSS optical spectrum was first examined for evidence of a low mass main-sequence companion. An increase in flux of the spectral energy distribution towards longer wavelengths or spectral features indicative of $\mathrm{M}$ dwarfs (e.g. $\mathrm{H} \alpha$ emission and/or $\mathrm{TiO}$ absorption bands) were searched for by eye. Empirical models for low mass sub-stellar objects were then added to a white dwarf synthetic spectrum and these composites were compared to UKIDSS photometry to obtain an approximate spectral type for the potential companion.

For candidates white dwarfs with $T_{\text {eff }}>16,000 \mathrm{~K}$ pure-H (DA) or pure-HE (DB/DO) synthetic spectra were calculated at the effective temperatures and surface gravities given 
Table 2.5. UKIDSS $Y J H K$ magnitudes of the candidate white dwarfs showing near-infrared excesses.

\begin{tabular}{|c|c|c|c|c|c|}
\hline SDSS J & WD & $\bar{Y}$ & $J$ & $\bar{H}$ & $\bar{K}$ \\
\hline "003902.47-003000.3 & - & $1818.70 \pm 0.06$ & $18.37 \pm 0.08$ & $1717.86 \pm 0.11$ & $\overline{17.41 \pm 0.10}$ \\
\hline $003923.04+003534.7$ & - & - & - & $17.48 \pm 0.05$ & $17.04 \pm 0.06$ \\
\hline $012032.27-001351.1$ & - & $19.25 \pm 0.08$ & $18.34 \pm 0.07$ & $17.82 \pm 0.07$ & $17.42 \pm 0.08$ \\
\hline $013532.98+144555.8$ & $132+145$ & $16.49 \pm 0.01$ & $16.24 \pm 0.01$ & $15.86 \pm 0.01$ & $15.56 \pm 0.02$ \\
\hline $023247.50-003909.3$ & - & - & $20.24 \pm 0.34$ & $18.80 \pm 0.20$ & $17.84 \pm 0.16$ \\
\hline $032317.00-002612.7$ & - & $15.03 \pm 0.00$ & $14.63 \pm 0.00$ & $14.08 \pm 0.00$ & $13.81 \pm 0.01$ \\
\hline $034221.55+005345.6$ & - & $16.48 \pm 0.01$ & $16.05 \pm 0.01$ & $15.45 \pm 0.01$ & $15.31 \pm 0.01$ \\
\hline $085956.47+082607.5$ & - & $18.33 \pm 0.03$ & - & $17.49 \pm 0.03$ & $17.09 \pm 0.08$ \\
\hline $090759.59+053649.7$ & - & $18.79 \pm 0.08$ & $18.66 \pm 0.08$ & $18.62 \pm 0.16$ & $18.40 \pm 0.19$ \\
\hline $092452.73+020712.2$ & $0922+006$ & - & - & $18.21 \pm 0.12$ & $17.63 \pm 0.11$ \\
\hline $092648.84+102828.8$ & - & $19.57 \pm 0.12$ & $19.19 \pm 0.16$ & $18.74 \pm 0.19$ & $18.29 \pm 0.19$ \\
\hline $100259.88+093950.0$ & - & $19.30 \pm 0.08$ & $19.19 \pm 0.13$ & $18.55 \pm 0.18$ & $18.55 \pm 0.22$ \\
\hline $101642.93+044317.7$ & - & - & - & $17.73 \pm 0.09$ & $17.59 \pm 0.09$ \\
\hline $103220.19+011227.0$ & - & _ & - & & $15 \pm 0.07$ \\
\hline $103448.92+005201.4$ & $1032+011$ & - & - & $18.20 \pm 0.11$ & $18.03 \pm 0.14$ \\
\hline $103736.75+013912.2$ & - & - & - & $17.25 \pm 0.06$ & $17.12 \pm 0.07$ \\
\hline $104933.58+022451.7$ & - & - & - & $17.80 \pm 0.09$ & $17.13 \pm 0.06$ \\
\hline $105332.62+020126.2$ & - & - & - & $17.26 \pm 0.05$ & $17.06 \pm 0.07$ \\
\hline $113416.09+0552$ & - & - & - & $18.10 \pm($ & $94 \pm 0.20$ \\
\hline $115808.44-012312.9$ & $1155-011$ & $18.26 \pm 0.03$ & $83 \pm 0$ & $17.22 \pm 0.03$ & $16.89 \pm 0.06$ \\
\hline $121209.31+013627.7$ & - & $18.04 \pm 0.04$ & $17.85 \pm 0.05$ & $17.21 \pm 0.05$ & $16.21 \pm 0.03$ \\
\hline $125044.42+154957.4^{1}$ & $1248+161$ & - & $16.61 \pm 0.02$ & $16.14 \pm 0.02$ & $15.00 \pm 0.01$ \\
\hline $122859.92+104033.0$ & - & $16.86 \pm 0.01$ & $16.89 \pm 0.02$ & $16.84 \pm 0.02$ & $16.42 \pm 0.04$ \\
\hline $132044.68+001855.0$ & $1318+005$ & $17.61 \pm 0.02$ & $17.62 \pm 0.04$ & $17.76 \pm 0.11$ & $17.38 \pm 0.11$ \\
\hline $132925.21+123025.4$ & - & $17.07 \pm 0.01$ & $16.66 \pm 0.01$ & $16.27 \pm 0.02$ & $15.98 \pm 0.02$ \\
\hline $154431.47+060104.3$ & - & $17.96 \pm 0.03$ & $17.83 \pm 0.04$ & $17.64 \pm 0.05$ & $17.64 \pm 0.10$ \\
\hline $155720.77+091624.7$ & - & $18.82 \pm 0.04$ & $18.82 \pm 0.06$ & $19.03 \pm 0.14$ & $18.34 \pm 0.15$ \\
\hline $220841.63-000514.5$ & - & $18.16 \pm 0.04$ & $17.48 \pm 0.03$ & $17.06 \pm 0.05$ & $16.59 \pm 0.04$ \\
\hline $222030.68-004107.9$ & - & $16.95 \pm 0.01$ & $16.75 \pm 0.02$ & $16.44 \pm 0.03$ & $16.36 \pm 0.04$ \\
\hline $222551.65+001637.7$ & - & $18.59 \pm 0.05$ & $18.51 \pm 0.07$ & $18.15 \pm 0.13$ & $17.60 \pm 0.13$ \\
\hline $233345.97-000843.0$ & - & $19.36 \pm 0.08$ & $18.80 \pm 0.10$ & $18.22 \pm 0.13$ & $17.67 \pm 0.13$ \\
\hline
\end{tabular}

${ }^{1}$ Identified in Section 2.5.2 
in Table 2.6 using the TLUSTY and SYNSPEC atmospheric and spectral synthesis codes. Below $\lesssim 16000 \mathrm{~K}$ convection becomes a significant factor in calculating white dwarf atmospheric models. For these stars blackbody models were generated instead at the temperatures given in Table 2.6. The synthetic fluxes were then normalised to the Sloan $i^{\prime}$ magnitude of the corresponding white dwarf (Table 2.6). The $i^{\prime}$-band was chosen for this work to avoid key absorption lines in the white dwarf's optical spectrum.

The empirical models for the candidate secondary stars were constructed using the NIR spectra of late M dwarfs from the IRTF spectral library (Cushing et al. 2005, Rayner et al. in prep.), and L and T-dwarfs presented by McLean et al. (2001). To extend the L and T-dwarf data out to $2.4 \mu \mathrm{m}$ they were appended to sections of CGS4 spectra obtained by Leggett et al. (2001) and Geballe et al. (2002).

In order to obtain an initial estimate on an approximate spectral type of a putative companion, an absolute $K$-band magnitude was calculated for each candidate system. This was achieved by subtracting the model predicted $K$-band flux from the observed UKIDSS $K$-band flux, and then scaling to the estimated distance of the white dwarf (Table 2.9). This value was compared to observed absolute $K$-band magnitudes of $\mathrm{M}, \mathrm{L}$ and T-dwarfs, which show a trend distinctive enough to separate the boundary regions of these three spectral types (Figure 2.6). Due to errors in temperature, surface gravity and therefore distance, estimates of the companion spectral types are likely to lie within \pm 1 spectral types of the best fitting composite white dwarf + companion model spectrum. 
Table 2.6. Physical parameters of the candidate white dwarfs showing near-infrared excesses. Temperatures and surface gravities are from Eisenstein et al. (2006) unless otherwise stated.

\begin{tabular}{|c|c|c|c|c|}
\hline SDSS J & WD & $g^{\prime}$ & $T_{\text {eff }}(\mathrm{K})$ & $\log g$ \\
\hline 003902.47-003000.3 & - & 19.12 & $1212314 \pm 268$ & $7.39 \pm 0.08$ \\
\hline $003923.04+003534.7$ & - & 19.20 & $10080 \pm 81$ & $7.99 \pm 0.10$ \\
\hline $012032.27-001351.1$ & - & 20.23 & $10487 \pm 194$ & $7.91 \pm 0.22$ \\
\hline $013532.98+144555.8$ & $0132+145$ & 16.96 & $8083 \pm 22$ & $8.08 \pm 0.04$ \\
\hline 023247.50-003909.3 & - & 20.12 & $9898 \pm 161$ & $8.04 \pm 0.23$ \\
\hline $032317.00-002612.7$ & - & 20.05 & $22193 \pm 1446$ & $7.10 \pm 0.19$ \\
\hline $034221.55+005345.6$ & - & 19.00 & $23035 \pm 684$ & $6.97 \pm 0.08$ \\
\hline $085956.47+082607.5$ & - & 18.75 & $15623 \pm 237$ & $7.44 \pm 0.06$ \\
\hline $090759.59+053649.7$ & - & 18.87 & $19474 \pm 293$ & $7.82 \pm 0.05$ \\
\hline $092452.73+020712.2$ & $0922+006$ & 18.96 & $15231 \pm 147^{1}$ & $8.49 \pm 0.10^{1}$ \\
\hline $092648.84+102828.8$ & - & 20.49 & $13214 \pm 935$ & $8.11 \pm 0.29$ \\
\hline $100259.88+093950.0$ & - & 19.58 & $22331 \pm 435$ & $7.87 \pm 0.06$ \\
\hline $101642.93+044317.7$ & - & 18.98 & $27039 \pm 276$ & $7.43 \pm 0.04$ \\
\hline $103220.19+011227.0$ & - & 19.28 & $8901 \pm 93$ & $7.71 \pm 0.18$ \\
\hline $103448.92+005201.4$ & $1032+011$ & 19.15 & $9904 \pm 109$ & $8.13 \pm 0.15$ \\
\hline $103736.75+013912.2$ & - & 18.81 & $16300 \pm 221$ & $7.73 \pm 0.05$ \\
\hline $104933.58+022451.7$ & - & 19.19 & $7590 \pm 96$ & $7.76 \pm 0.32$ \\
\hline $105332.62+020126.2$ & - & 19.06 & $7530 \pm 90$ & $8.14 \pm 0.30$ \\
\hline $113416.09+055227.2$ & - & 19.58 & $24184 \pm 650$ & $7.85 \pm 0.08$ \\
\hline $115808.44-012312.9$ & $1155-011$ & 19.62 & $\sim 13300^{2}$ & - \\
\hline $121209.31+013627.7$ & - & 17.97 & $\sim 10000^{3}$ & $\sim 8.0^{3}$ \\
\hline $125044.42+154957.4$ & $1248+161$ & 18.36 & $\sim 10000^{4}$ & - \\
\hline $122859.92+104033.0$ & - & 16.91 & $22125 \pm 136$ & $8.22 \pm 0.02$ \\
\hline $132044.68+001855.0$ & $1318+005$ & 17.69 & $19649 \pm 130$ & $8.36 \pm 0.02$ \\
\hline $132925.21+123025.4$ & - & 17.71 & $13537 \pm 138$ & $7.48 \pm 0.02$ \\
\hline $154431.47+060104.3$ & - & 18.49 & $7946 \pm 66$ & $8.39 \pm 0.15$ \\
\hline $155720.77+091624.7$ & - & 19.09 & $21990 \pm 403$ & $7.67 \pm 0.06$ \\
\hline $220841.63-000514.5$ & - & 19.19 & $9224 \pm 81$ & $8.28 \pm 0.12$ \\
\hline $222030.68-004107.9$ & - & 17.38 & $8037 \pm 31$ & $8.28 \pm 0.07$ \\
\hline $222551.65+001637.7$ & - & 18.94 & $10640 \pm 94$ & $8.16 \pm 0.09$ \\
\hline 233345.97-000843.0 & - & 20.65 & $11198 \pm 433$ & $8.35 \pm 0.29$ \\
\hline
\end{tabular}

${ }^{1}$ Helium fit.

${ }^{2}$ Blackbody fit of SDSS photometry.

${ }^{3}$ Farihi et al. (2008)

${ }^{4}$ Vanlandingham et al. (2005) 


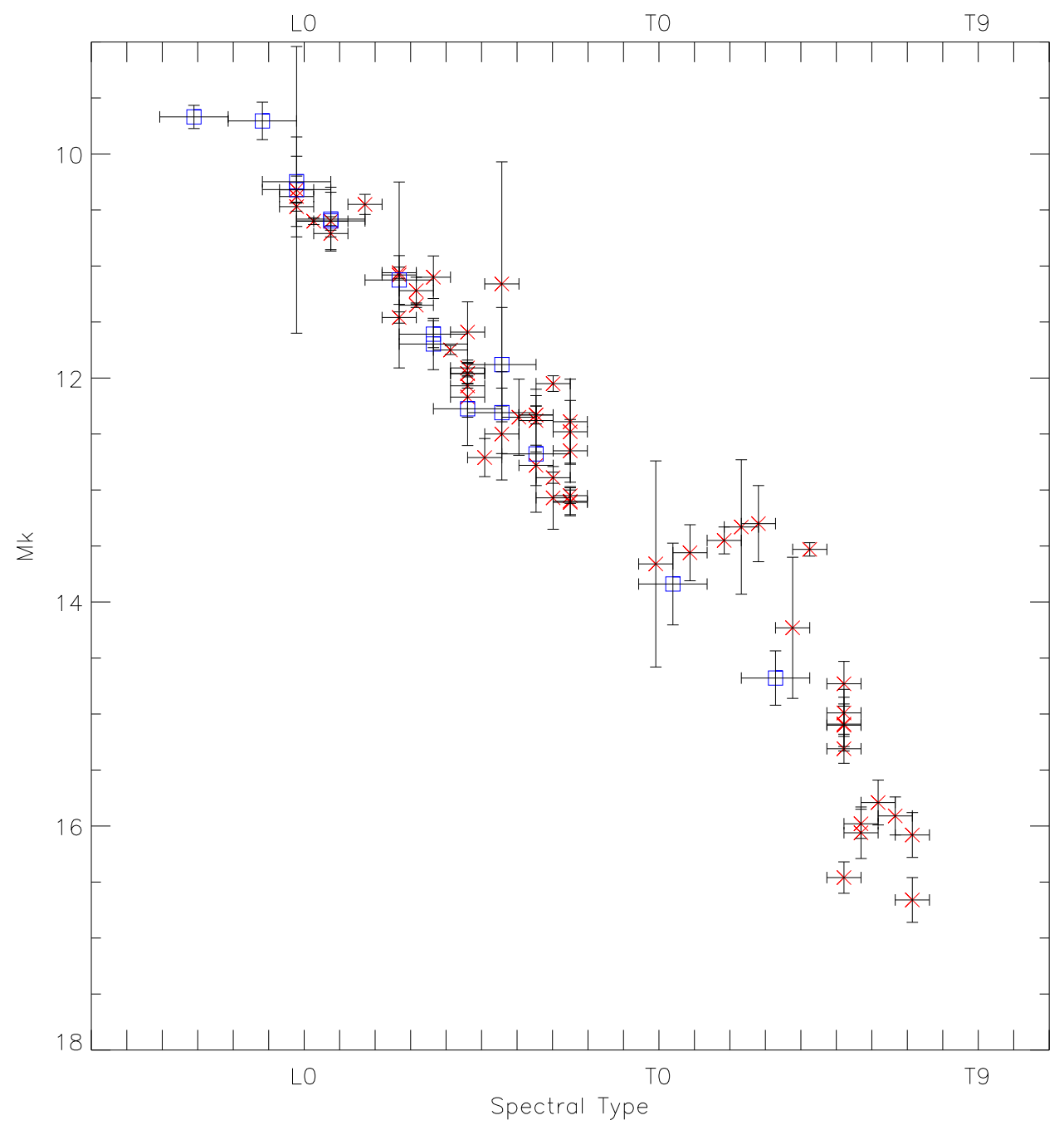

FIgURE 2.6. Absolute $K$-band photometry verses spectral type for $\mathrm{L}$ and T-dwarfs (Red: Reproduced from Vrba et al. 2004). Also plotted are the predicted absolute magnitudes of the candidate companions in this survey (blue). 


\subsubsection{Identifying Debris Disks}

As was discussed in Section 1.3, debris disks can be identified through an excess in the $K$-band magnitude of the white dwarf. Using the following method, blackbody models were used to obtain an upper limit on the temperature of the potential disk: a blackbody model was generated at the temperature of white dwarf and scaled appropriately. This model was then added to the white dwarf synthetic spectrum to create a combined white dwarf + blackbody model (WD+BB). This combined model represents a white dwarf with a cool $(<1000 \mathrm{~K})$ disk. Starting at a temperature of $1000 \mathrm{~K}$, the temperature of the secondary blackbody was reduced in steps of $100 \mathrm{~K}$ until the combined WD+BB model matched the $H$ and $K$-band photometry. Here it is assumed there is no excess at $H$.

The higher the temperature of the debris the disk, the shorter the wavelength at which its spectral energy distribution will peak. Therefore, as higher temperature blackbodies are added to the white dwarf's model spectrum, an excess will eventually be be seen in the $H$, as well as the $K$-band. Hence, the temperature found previously can be adopted as an upper limit, as lower temperature disk combinations can also produce models which match the $K$-band photometry alone. NIR $K$-band spectroscopy or mid-infrared photometry would be needed to more accurately constrain the temperature of any debris disk containing system. 


\subsection{Follow-up Near-infrared Photometric Observations}

For a small number of sources, follow-up NIR photometry was obtained to independently measure their $J H K$ magnitudes. SDSS J222551.65+001637.7 was observed on October $2^{\text {nd }}\left(\right.$ at $\left.K_{\mathrm{s}}\right)$ and $3^{\text {rd }}(J \& H) 2007$ with the $3.5 \mathrm{~m}$ New Technology Telescope (NTT) and the Son-of-ISAAC (SOFI) Imager. Individual exposure times were 15 secs, 12 secs and 8 secs for the $J, H$ and $K_{\mathrm{s}}$-bands respectively, with a total exposure time in each band of 150 secs, 288 secs and 240 secs. The weather was clear with seeing of $J=1.3$ " on the first night and $K_{\mathrm{s}}=1.4$ " on the second night.

SDSS J003902.47-003000.3 was observed also with the NTT + SOFI on October $25^{\text {th }}$ 2007 in $J, H \& K_{\mathrm{s}}$. Individual exposure times were 15 secs, 12 secs and 12 secs for the $J, H$ and $K_{\mathrm{s}}$-bands respectively, with a total exposure time in each band of 75 secs, 144 secs and 144 secs. The weather was clear and photometric with seeing at $K_{\mathrm{s}}=0.7$ '.

WD 1318+005 was observed at the 3.9m Anglo-Australian Telescope (AAT) with the IRIS2 NIR imager on $27^{\text {th }}$ August 2007. Individual exposures times were 5 secs in the $K$-band with a total exposure time of 75 secs. The average seeing over the course of the observations was $=2.4$ ".

Nodding was used for all observations to ease the removal of the NIR background. All the data were reduced automatically in the standard manner with the ORAC-DR package. The combined frames were photometrically calibrated using UKIDSS sources in each field. Aperture photometry was performed interactively using the Starlink Gaia package. 


\subsection{Candidate UKIDSS Photometry}

For each candidate system with a putative unresolved stellar/substellar companion, an upper limit on the projected orbital separation has been estimated. This has been achieved by measuring the FWHM of the system in the bandpass where the contributions of flux from each object would be roughly equal (usually the $z^{\prime}$ or $J$-band depending on the availability of the data), and equating this to an orbital separation at the estimated distance to the white dwarf. In the few cases where the system has been resolved, the separation between the components has been measured directly and equated to an orbital separation.

For the few resolved systems, the probability of a chance alignment $\left(\mathrm{P}_{\mathrm{al}}\right)$ has been calculated by referring to the spatial number densities of M, L and T dwarfs (Table 2.7; Caballero, Burgasser \& Klement 2008). The expected number of stars with the spectral type assigned to putative companion is then calculated within a region of space defined by the errors on the white dwarf's distance and the estimated projected orbital separation. This has to be multiplied by the total sample size in order to take the size of the survey into account.

A number of examples have been discussed in detail in the following sections. A complete summary of results is given in Table 2.8 and Figures included in Appendix $A$. 
Table 2.7. The number density $n$ of spectral types ranging from M3-T8 V. Table from Caballero, Burgasser \& Klement (2008)

\begin{tabular}{cccccc}
\hline Spectral Type & $\begin{array}{c}M_{I} \\
(\mathrm{mag})\end{array}$ & $\begin{array}{c}I-J \\
(\mathrm{mag})\end{array}$ & $\begin{array}{c}J-K_{s} \\
(\mathrm{mag})\end{array}$ & $\begin{array}{c}n \\
\left(10^{-3} \mathrm{pc}^{-3}\right)\end{array}$ & $\begin{array}{c}d_{I=25} \\
(\mathrm{pc})\end{array}$ \\
\hline \hline M3-4 V & 9.07 & 1.83 & 0.84 & 19.6 & 7700 \\
M4-5 V & 10.14 & 1.80 & 0.86 & 16.3 & 5700 \\
M5-6 V & 11.91 & 2.47 & 0.90 & 9.81 & 3100 \\
M6-7 V & 12.90 & 2.72 & 0.93 & 4.90 & 2200 \\
M7-8 V & 14.16 & 3.24 & 0.98 & 1.907 & 1300 \\
M8-9 V & 14.68 & 3.54 & 1.06 & 1.285 & 1100 \\
M9-L0 V & 15.23 & 3.80 & 1.12 & 0.768 & 840 \\
L0-1 V & 15.27 & 3.55 & 1.27 & 0.637 & 820 \\
L1-2 V & 15.69 & 3.50 & 1.38 & 0.861 & 680 \\
L2-3 V & 16.19 & 3.62 & 1.43 & 1.000 & 550 \\
L3-4 V & 16.67 & 3.72 & 1.49 & 0.834 & 450 \\
L4-5 V & 17.14 & 3.81 & 1.54 & 0.819 & 360 \\
L5-6 V & 17.61 & 3.90 & 1.60 & 0.869 & 290 \\
L6-7 V & 18.07 & 4.06 & 1.66 & 0.785 & 240 \\
L7-8 V & 18.52 & 4.12 & 1.71 & 0.644 & 190 \\
L8-9 V & 18.90 & 4.22 & 1.81 & 0.462 & 160 \\
L9-T0 V & 18.95 & 4.40 & 1.73 & 0.308 & 160 \\
T0-1 V & 19.01 & 4.63 & 1.58 & 0.220 & 160 \\
T1-2 V & 19.04 & 4.94 & 1.17 & 0.241 & 150 \\
T2-3 V & 19.04 & 5.19 & 0.81 & 0.406 & 150 \\
T3-4 V & 19.07 & 5.23 & 0.51 & 0.788 & 150 \\
T4-5 V & 19.22 & 5.01 & 0.29 & 1.42 & 140 \\
T5-6 V & 19.61 & 4.76 & 0.11 & 2.38 & 120 \\
T6-7 V & 20.35 & 5.25 & 0.00 & 3.45 & 85 \\
T7-8 V & 21.60 & 5.50 & -0.06 & 4.82 & 48 \\
\hline
\end{tabular}




\subsubsection{Examples of White Dwarfs with a NIR Excess Indicative of a Low Mass Companion}

\section{SDSS J003902.47-003000.3: A White Dwarf with an Unresolved Low Mass Stellar Companion}

SDSS J0039-003's optical spectrum shows no obvious evidence for an M-dwarf companion. EIS06 give the white dwarf physical parameters as $T_{\text {eff }}=12,314 \pm 268 \mathrm{~K}$ and $\log g=7.39 \pm 0.08$ from an automated fit to the optical spectrum. When compared to an atmospheric model at this temperature and gravity, a large excess is visible in the SDSS $z^{\prime}$-band (Figure 2.7), with a clear strong excess emission in the UKIDSS $Y J H K$-band photometry. The predicted absolute $K$-band photometry of the potential companion suggests a spectral type of M7. However, it was found that a composite WD+L0 spectrum is the best fit to the UKIDSS photometry.

I first identified this result in UKIDSS DR2 and follow-up $J H K$-band photometry was obtained using SOFI on the NTT in October 2007 (Section 2.4). The SOFI photometry confirms the presence of a NIR excess and agrees that the companion is likely of spectral type L0. The UKIDSS mergedClass statistic for SDSS J0039-003 indicates the system is a point source, with a measured FWHM of 0.73 " in the $J$-band. This equates to a projected orbital separation of $<284 \mathrm{AU}$ for the secondary at the estimated distance to the white dwarf (Table 2.9). However, the low mass of the white dwarf $\left(0.34 \pm 0.02 M_{\odot}\right)$ suggests an accelerated evolution caused by a secondary component, and so it is likely that the companion is close. If this is the case, a discrepancy in estimated spectral type of the secondary may arise from variability in the NIR photometry, due to night and dayside 


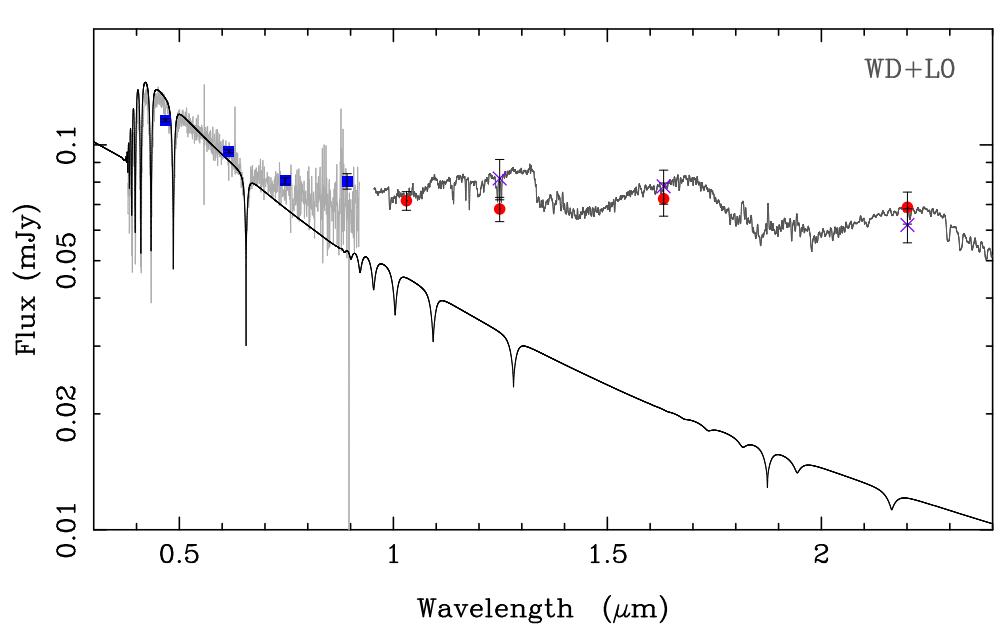

FIGURE 2.7. SDSS J003902.47-003000.3 model spectrum (black solid) with SDSS griz' (blue), UKIDSS $Y J H K$ (red) and NTT $J H K$ photometry (purple). Also shown are the SDSS spectrum (light grey) and a composite WD+L0 spectrum (dark grey).

heating of the companions atmosphere. NIR time series photometry is needed to confirm this scenario.

\section{SDSS J012032.27-001351.1: A White Dwarf with a resolved Low Mass Stellar Companion}

SDSS J0120-001 was classified as a DA white dwarf by EIS06 with $T_{\text {eff }}=10,487 \pm$ $194 \mathrm{~K}$ and $\log g=7.91 \pm 0.23$. However, the SDSS finder image shows a faint red background object which may have contaminated the optical spectrum to some extent (Figure 2.8). An excess is clearly seen in the UKIDSS photometry, most likely due to this background source. Indeed, a closer inspection of the SDSS and UKIDSS images (Figure $2.9 \& 2.10$ ) reveals that the white dwarf has not been detected in the the NIR and hence it is only the flux from the nearby red object that is being measured. The two components are separated by $\approx 2$ " equating to a projected orbital separation of 


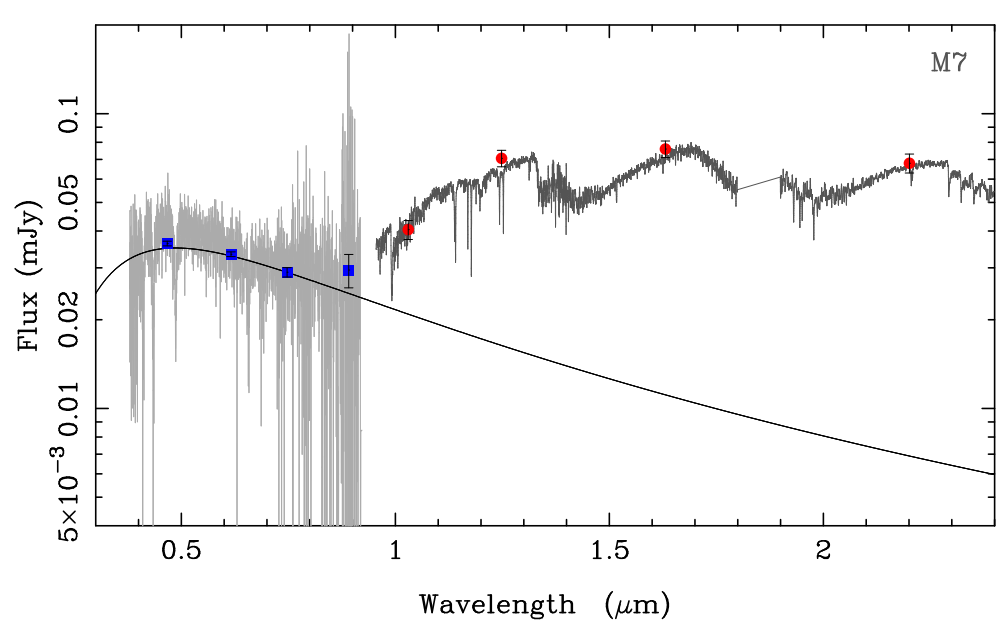

FIGURE 2.8. SDSS J012032.27-001351.1 model spectrum (black solid) with SDSS griz' (blue) and UKIDSS $Y J H K$ photometry (red). Also shown is the SDSS spectrum (light grey) and an M7 spectrum scaled to the $K$-band magnitude of the putative secondary.

$\approx 910 \mathrm{AU}$. If the two are associated then the UKIDSS photometry is best matched by an M7 dwarf scaled to the estimated distance of the white dwarf.

The expected number density of stars of spectral type M6-M8 V is $6.8 \times 10^{-3} \mathrm{pc}^{-3}$. Therefore, assuming an error of \pm 1 on the spectral type of the red object, the expected number of stars of spectral type M6-M8 V in a volume of space defined by the errors of the white dwarf's distance $(140 \mathrm{pc})$ and the projected separation is $\mathrm{P}_{\mathrm{al}}=5.8 \times 10^{-2}$. Thus, this could be the widest white dwarf + M-dwarf binary to be identified in this work. Followup proper motion measurements are required to assess the association of the two objects, as a background giant star cannot be discounted at this stage. 


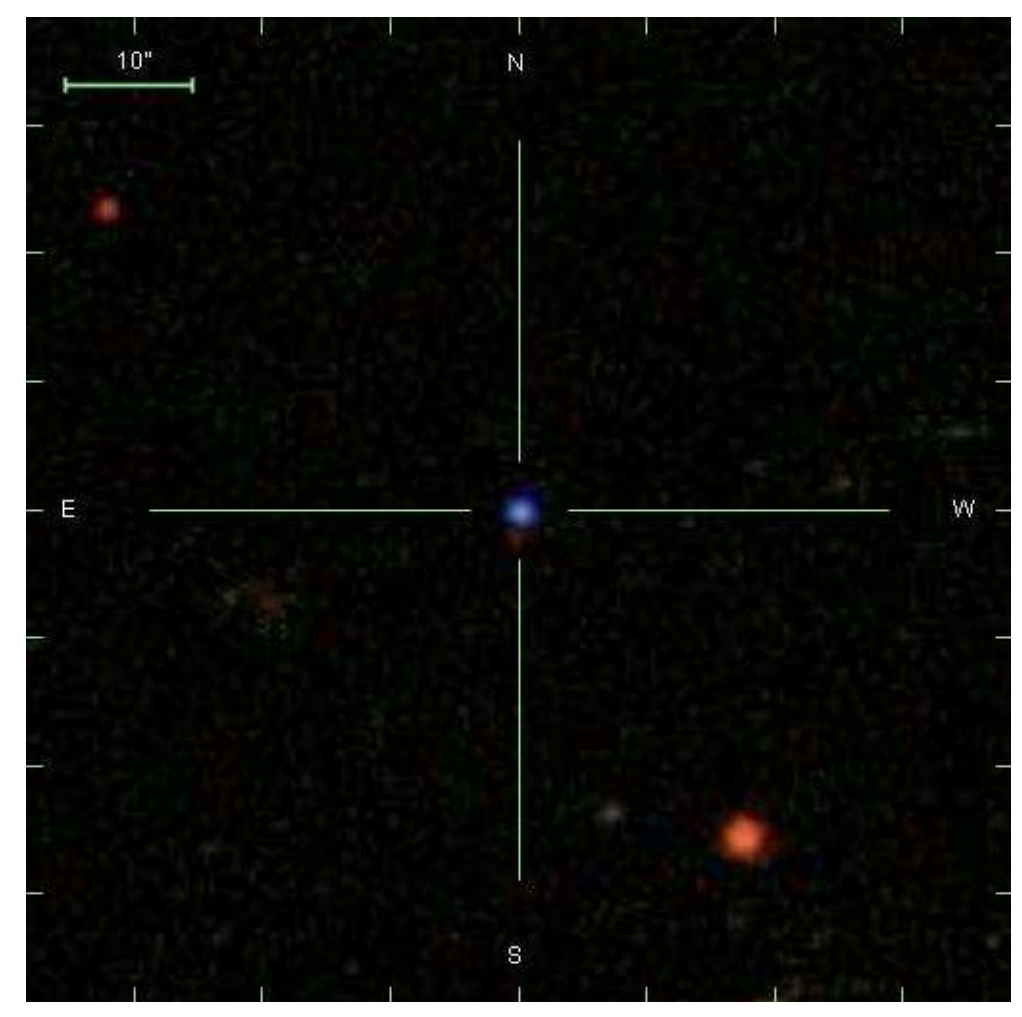

FIGURE 2.9. SDSS finder image of SDSS J012032.27-001351.1. A red object can be seen just to the south of the blue white dwarf.

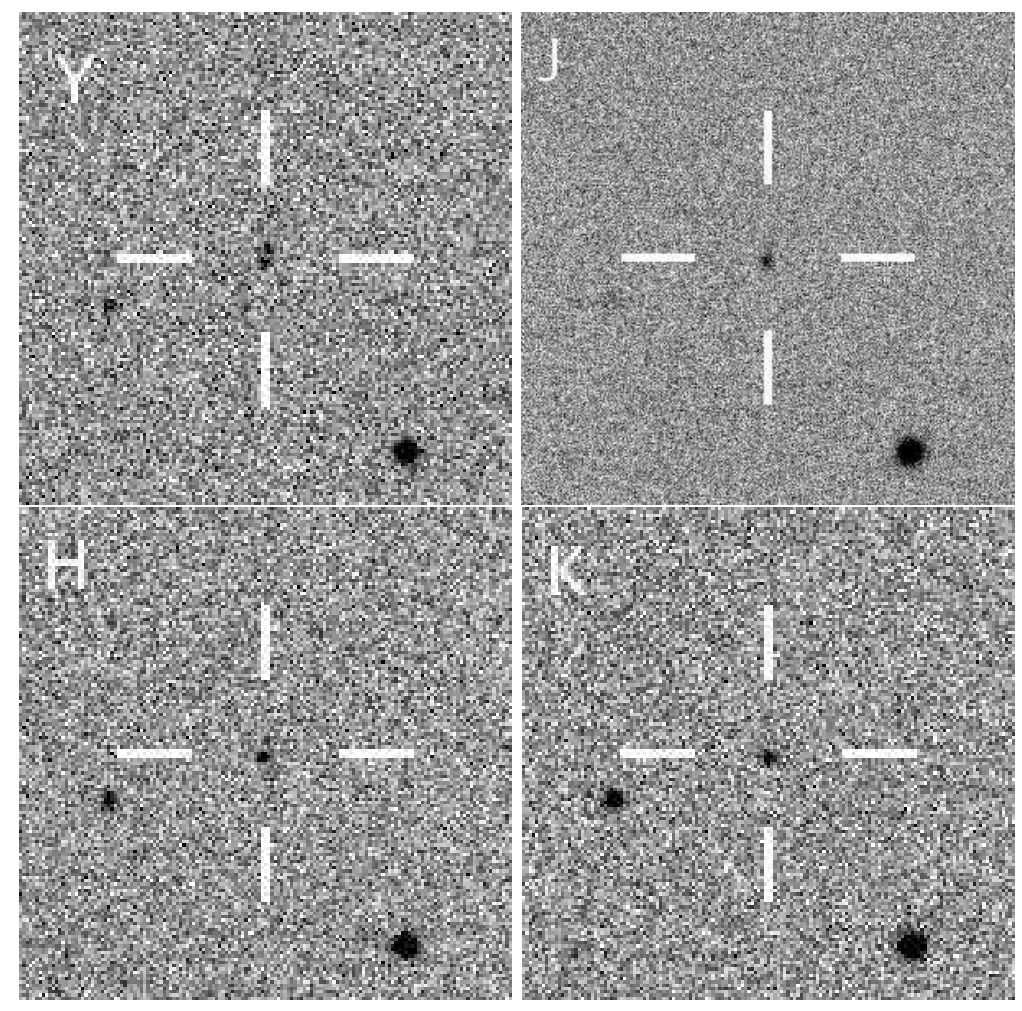

FIGURE 2.10. UKIDSS $Y J H K$ images of SDSS J012032.27-001351.1. Both objects from the SDSS image can be seen in the UKIDSS $Y$-band, with only the southern most object appearing in the $J H K$ images. 


\section{SDSS J013532.98+144555.8 = NLTT 5306: A White Dwarf with an Unresolved Brown Dwarf companion}

NLTT 5306 is classified by EIS06 as a DA white dwarf with $T_{\text {eff }}=8100 \pm 20 \mathrm{~K}$ and $\log g=8.08 \pm 0.04$. The SDSS optical photometry and spectrum (Figure 2.11) show no sign of an excess due to a stellar companion. However, an excess is clearly visible in the UKIDSS photometry, with the predicted absolute $K$-band magnitude of the companion suggesting a spectral type of L6. A best fit is achieved with a composite blackbody + L5 spectrum. At the white dwarf age of 2.1 Gyr (Section 2.6), the DUSTY atmospheric models (Chabrier et al. 2000, Baraffe et al. 2002) predict a mass of $58 \pm 2 M_{\mathrm{J}}$. Therefore, it is likely SDSS $0135+144$ harbours a brown dwarf companion. The UKIDSS mergedClass statistic for NLTT 5306 indicates the system is a point source, with a measured FWHM of $0.96 "$ in the $J$-band. This equates to a projected orbital separation of $<57 \mathrm{AU}$ for the secondary at the estimated distance to the white dwarf (Table 2.9).

NLTT 5306 is known to have a high proper motion $\left(\mu_{\mathrm{abs}}=0.194\right.$ " $\mathrm{yr}^{-} 1$, Lépine \& Shara 2005). The star was identified in the both the first and second Palomar Sky Surveys (POSS I and POSS II), and a composite image was produced from the data (Figure 2.12). No background object can be seen to be present along the path of NLTT 5306, increasing the likelihood that this star has a secondary low mass companion. 


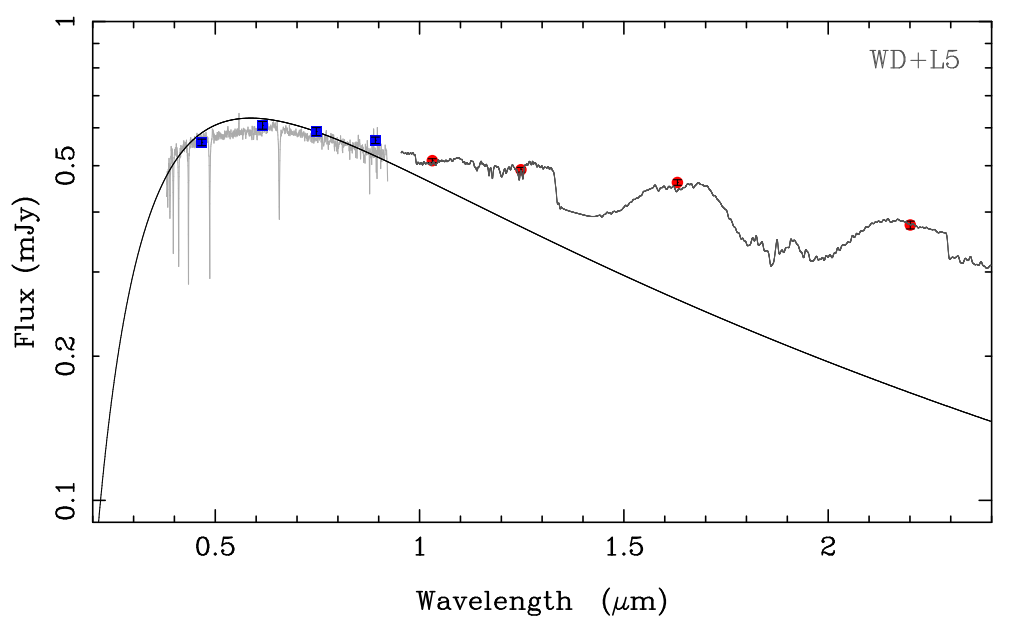

FIGURE 2.11. SDSS J013532.98+144555.8 model spectrum (black solid) with SDSS griz' (blue) and UKIDSS $Y J H K$ photometry (red). Also shown are the SDSS spectrum (light grey) and a composite WD+L5 spectrum (dark grey). 

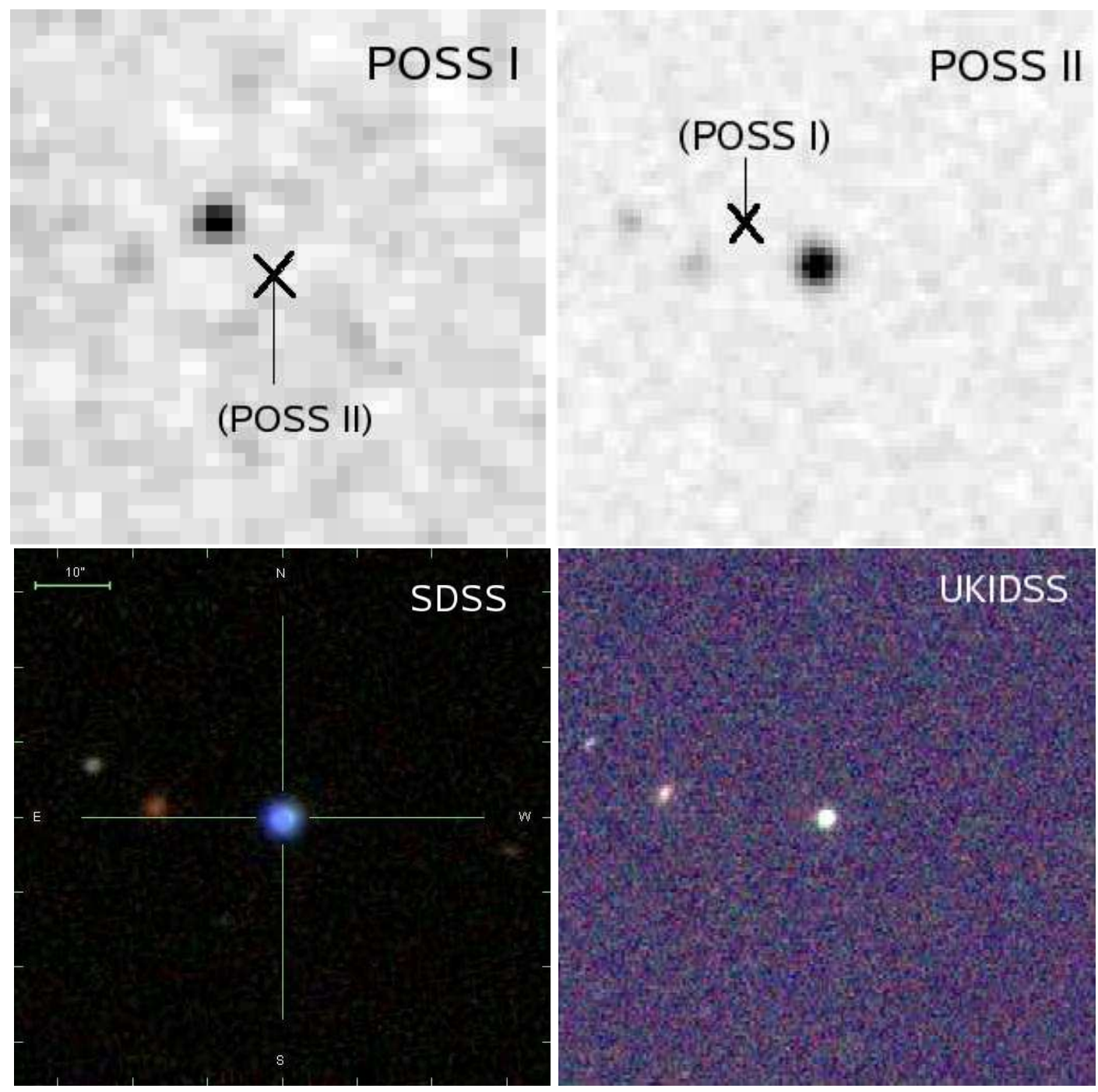

FIGURE 2.12. POSS I and POSS II images of NLTT 5306, with the current and prior positions of the star respectively marked. Also shown is the current epoch finder image from SDSS, and a composite $J H K$ image from the UKIDSS DR5. 
SDSS J023247.50-003909.3: Contamination from a Foreground/Background Source

SDSS J0232-003 is a DA white dwarf with EIS06 parameters of $T_{\text {eff }}=9898 \pm 161 \mathrm{~K}$ and $\log g=8.04 \pm 0.24$. The white dwarf has $i^{\prime}=20.11 \pm 0.03$ and so would be too faint to be detected by UKIDSS in the NIR. However, a secondary object was detected with a separation of 2.1" from SDSS J0232-003 (Figures 2.14 \& 2.15), equating to a projected separation of $700 \mathrm{AU}$. At the estimated distance to the white dwarf of $334 \pm 83 \mathrm{pc}$, the $K$ band magnitude of the potential secondary indicates a spectral type of M8-L2. However, it was found that a star of spectral type L3 was the best fit to the UKIDSS photometry (Figure 2.13). This difference in estimates could be due to the large uncertainties in the UKIDSS magnitudes and therefore distance estimate to the white dwarf. However, it is highly unlikely that an object of this spectral type would have been seen in the optical images. Therefore, it is more likely that this is a foreground or background object and so will be excluded from statistical analysis (Chapter 6).

SDSS J092452.73+020712.2 = WD 0922+006: A Helium Atmosphere White Dwarf with a Brown Dwarf Companion

WD 0922+006 is the only helium atmosphere (DB) white dwarfs in our list of candidates for low mass sub-stellar companions. A helium model atmosphere fit by EIS06 gives physical parameters of $T_{\text {eff }}=15,231 \pm 147 \mathrm{~K}$ and $\log g=8.49 \pm 0.10$. A blackbody model calculated using this temperature produces a good fit to both the SDSS photometry and spectrum (Figure 2.16). Two measurements exist in the UKIDSS database in close proximity to the position of the star, one with $Y J$-band photometry and the 


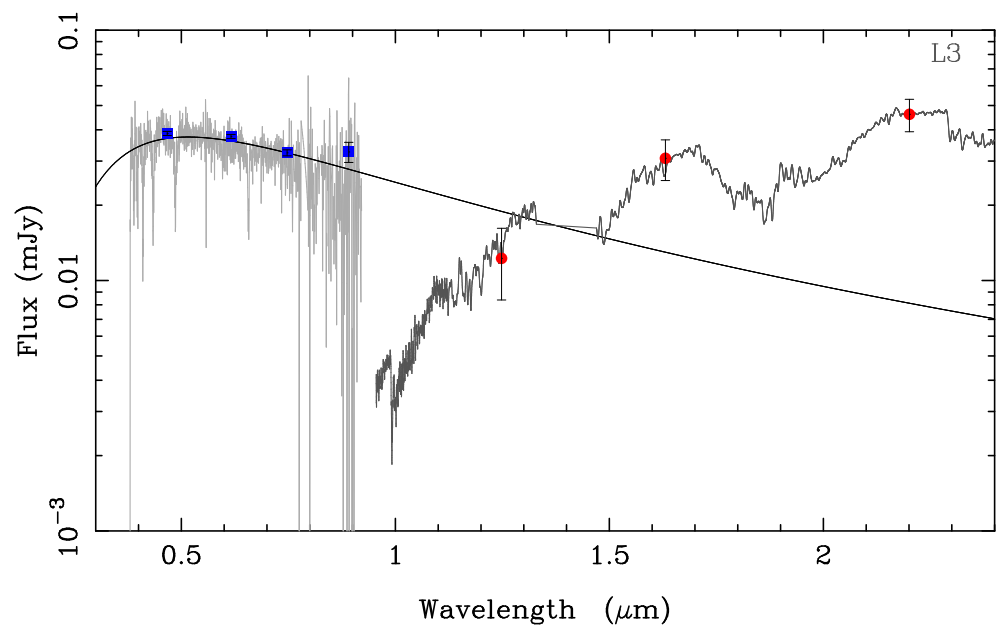

FIGURE 2.13. SDSS J023247.50-003909.3 model spectrum (black solid) with SDSS griz' (blue) and UKIDSS $J H K$ photometry (red). Also shown is the SDSS spectrum (light grey) and an L3 spectrum scaled to the $K$-band magnitude of the putative secondary. 


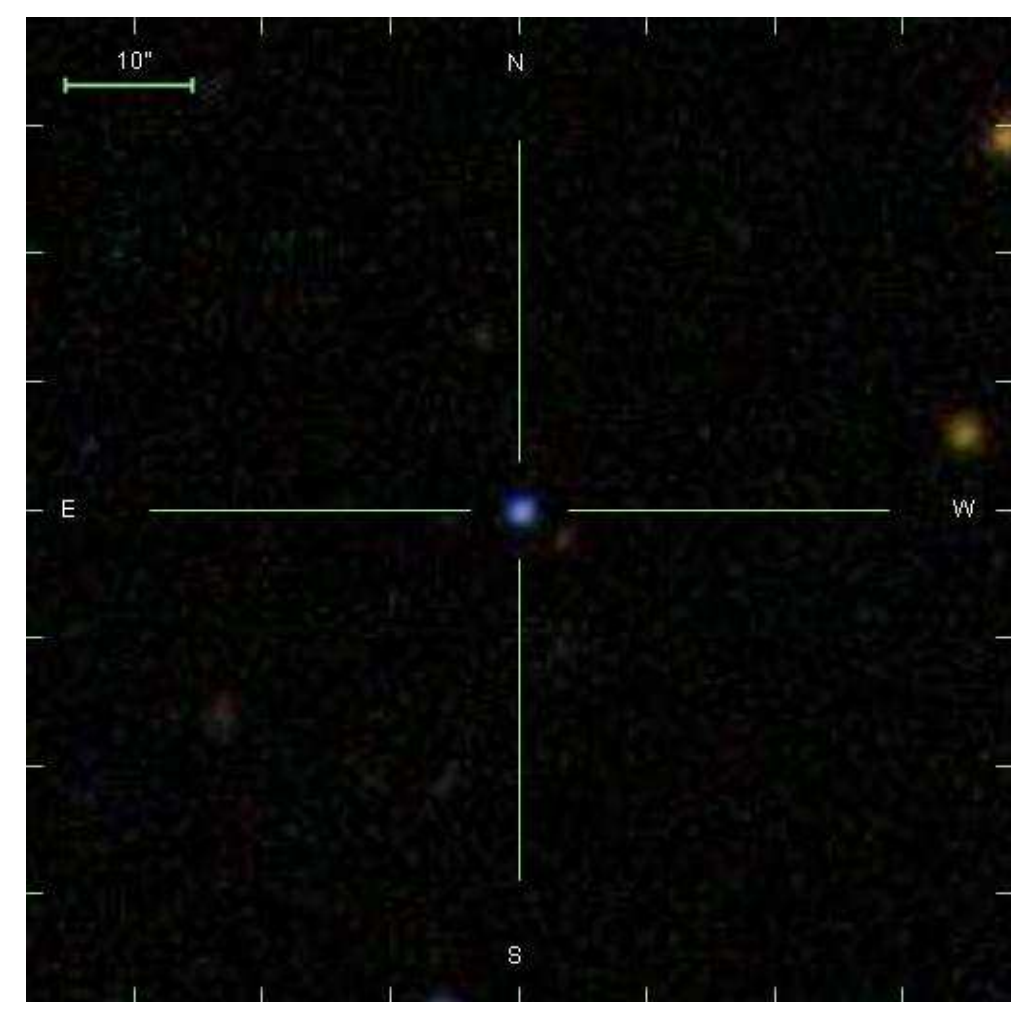

FIGURE 2.14. SDSS finder image of SDSS J023247.50-003909.3. A red object can be seen just to the south west of the blue white dwarf.

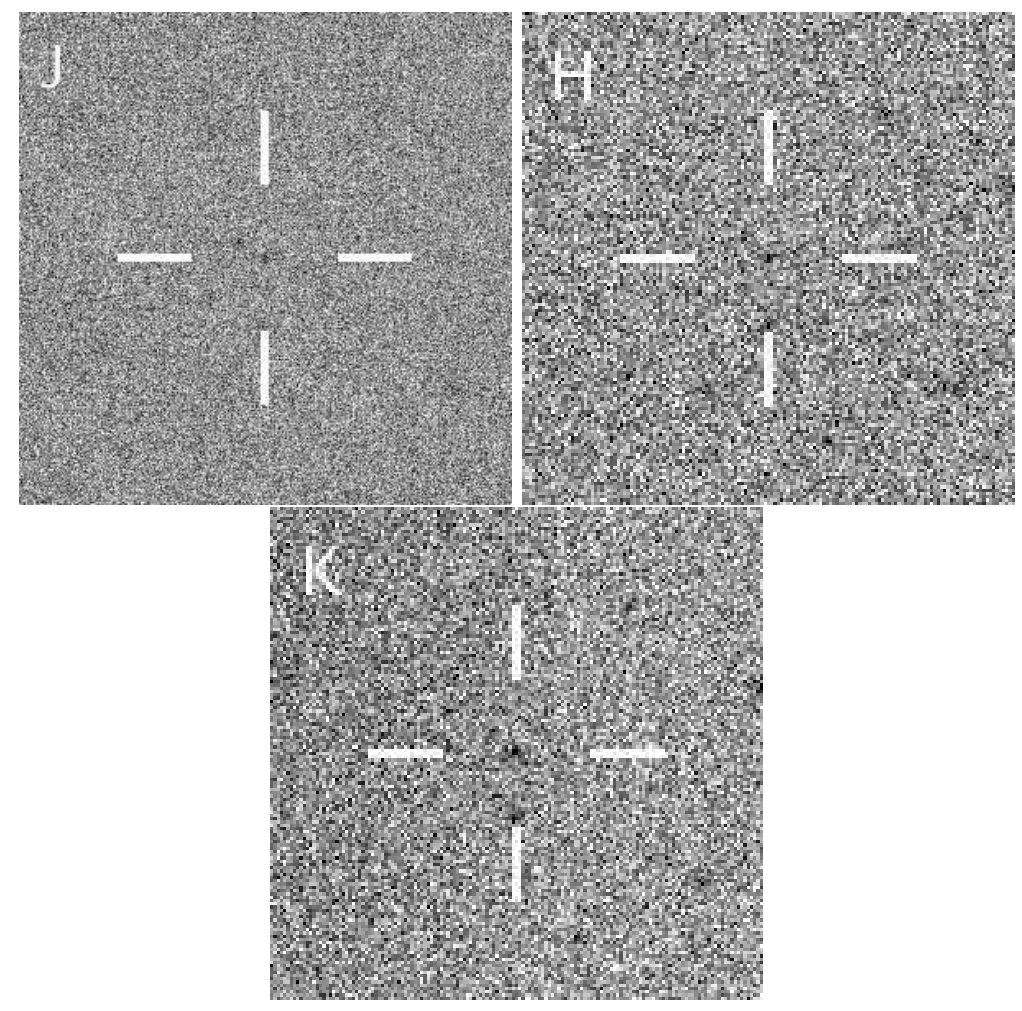

FIGURE 2.15. UKIDSS $J H K$ images of SDSS J023247.50-003909.3. The white dwarf has almost completely faded from view in the $J$-band image, with the red object completely dominating in the $H$ and $K$-bands. 
other $Y H K$-band. A closer inspection of the SDSS and UKIDSS images (Figures 2.17

\& 2.18) suggests a partially resolved system at the position of WD $0922+006$ with a separation of $\approx 1.1 "$. This equates to a projected orbital distance of $\approx 240 \mathrm{AU}$. The UKIDSS mergedClass statistic for WD $0922+006$ indicates the system is an extended source, and so it is likely that there is a cross contamination of the measured flux from both components.

The blackbody model fits the first set of $Y J$-band photometry, which is that of WD 0922 $+006 \mathrm{~A}$. The second set of measurements, the $Y H K$-band photometry, is that of the potential companion, with the predicted absolute $K$-band magnitude suggesting a spectral type of L3. An L4 spectrum normalised to the UKIDSS $K$-band photometry produces a reasonable fit to all the UKIDSS data for the potential secondary. This would be the lowest mass object to be found in a wide-orbit around a helium atmosphere white dwarf, and potentially the first DB + brown dwarf binary.

If an error of \pm 1 is assumed on the spectral type of the secondary then the expected number density of stars of spectral type L3-L5, is $1.65 \times 10^{-3} \mathrm{pc}^{-3}$. So the expected number of stars of these spectral types within a volume of space defined by the errors on the white dwarfs distance and the projected separation is $\mathrm{P}_{\mathrm{al}}=2.8 \times 10^{-7}$. Therefore, if this star is indeed confirmed to be a brown dwarf, then it is highly likely that the two objects are associated. A spectrum of the potential secondary is required to confirm the nature of the putative companion and proper motion measurements are required to confirm the two objects are associated. 


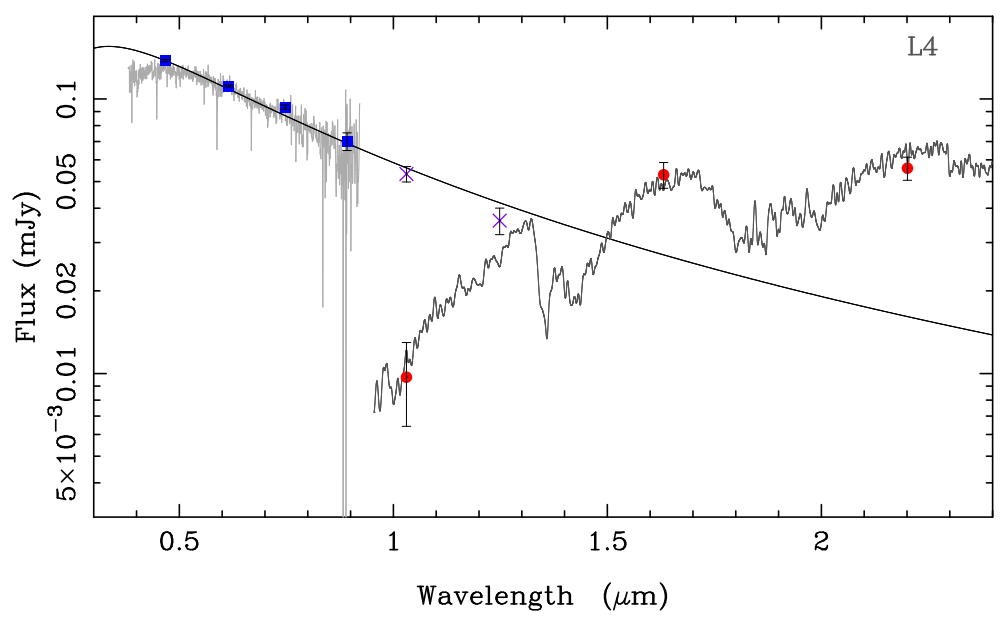

FIGURE 2.16. WD 0922+006 model spectrum (solid black) with SDSS griz' (blue) and UKIDSS $Y J H K$ photometry (purple: WD, red: putative companion). Also shown are the SDSS spectrum (light grey) and an L4 spectrum scaled to the $K$-band magnitude of the putative secondary (dark grey). 


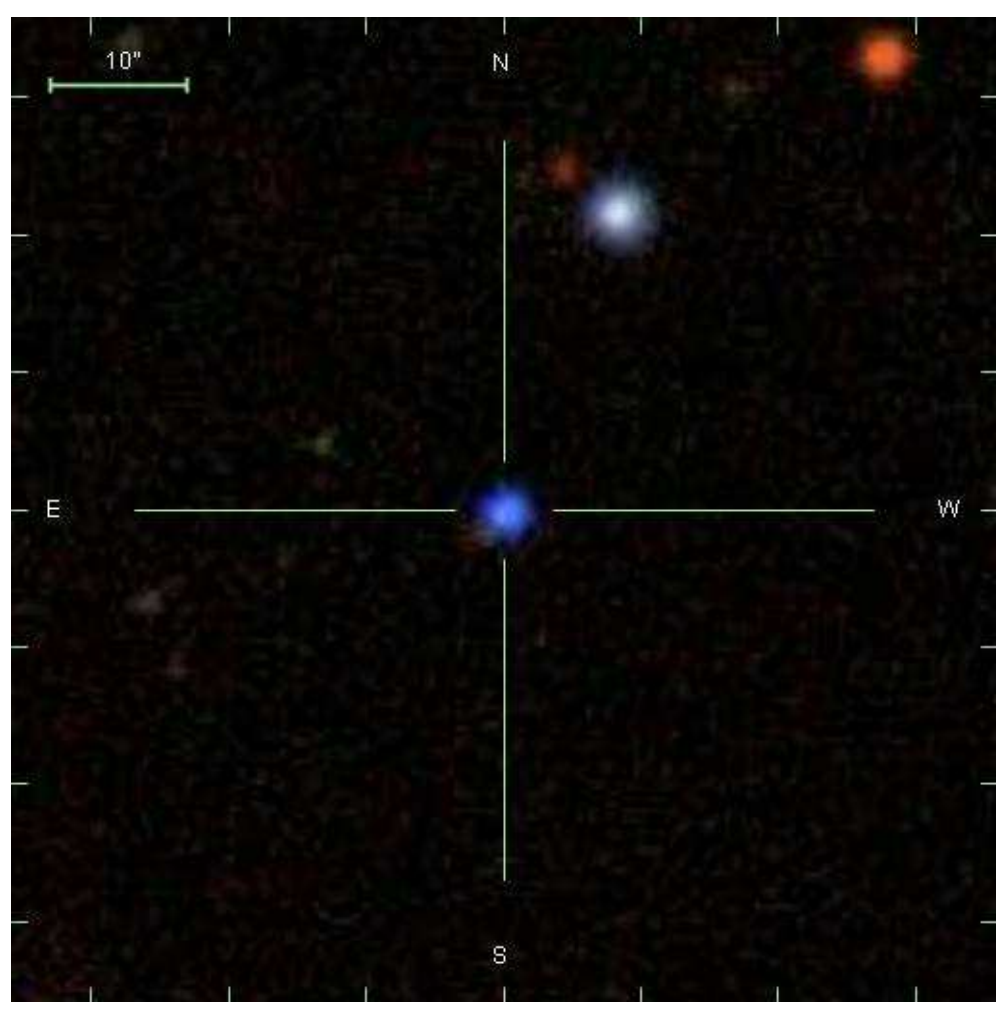

FIGURE 2.17. SDSS finder image of WD $0922+006$. There is a hint of possible red contamination in the south east corner of the blue white dwarf.

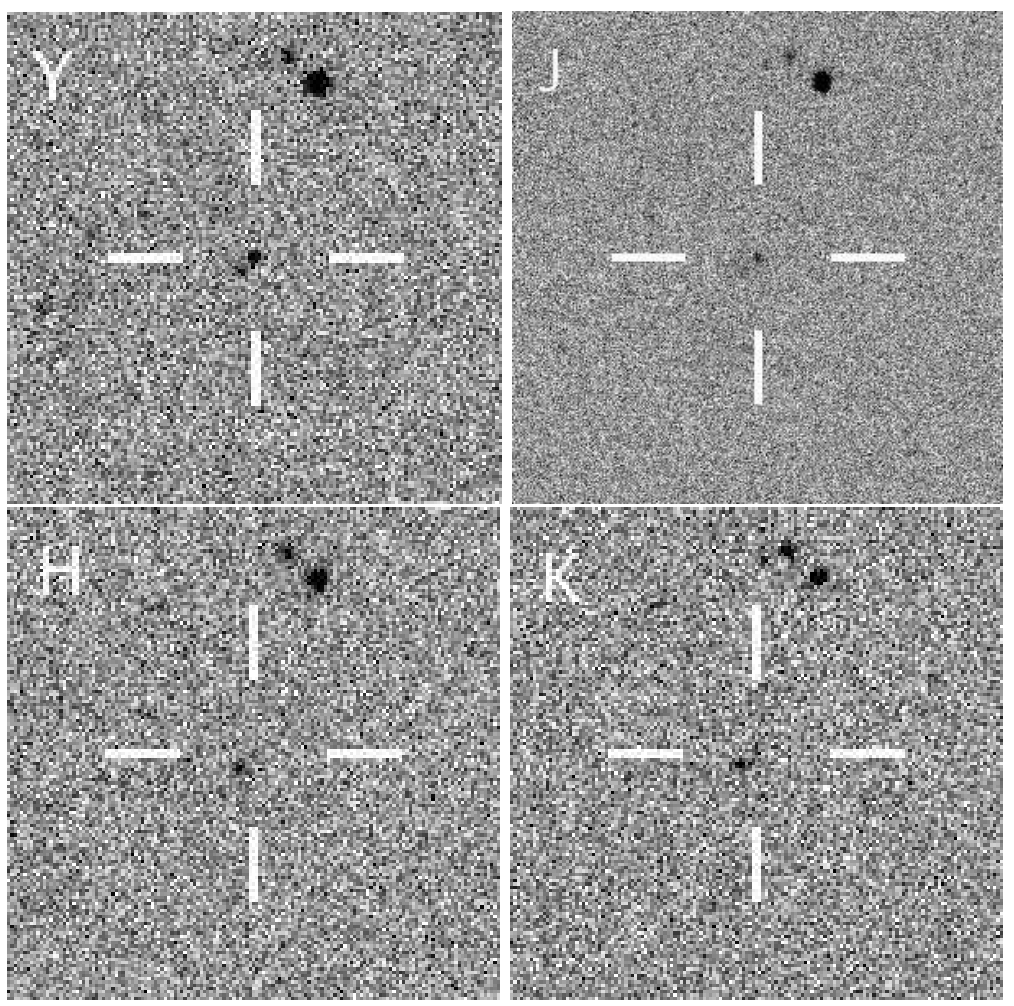

FIGURE 2.18. UKIDSS $Y J H K$ images of WD $0922+006$. The red object clearly begins to dominate at longer wavelengths. 
SDSS J154431.47+060104.3: A White Dwarf with an Unresolved T dwarf Companion

SDSS $1544+060$ shows no optical excess when compared to a blackbody model at $T_{\text {eff }}=$ $7946 \pm 66 \mathrm{~K}$, the temperature assigned by the EIS06 automated fit (Figure 2.19). An excess is clearly visible in each of the UKIDSS bands with the predicted absolute $K$-band magnitude of the secondary suggesting a companion type of early T. Each data point matches a composite WD+T3 model particularly well with the $J$-band photometry possibly indicating the detection of the $\mathrm{CH}_{4}$ absorption feature. If confirmed this would potentially be the first detection of a WD+dT dwarf binary. The UKIDSS mergedClass statistic for SDSS J1329+123 indicates the system is a point source, with a measured FWHM of 2.12" in the J-band. This equates to a projected orbital separation of $<190 \mathrm{AU}$ for the secondary at the estimated distance to the white dwarf (Table 2.9). If the system were in a close orbit the secondary would be the lowest object to have survived common envelope evolution. Spectroscopic confirmation is now urgently required for this potential new type of WD binary system, with follow up radial velocity measurements to assess the separation of components.

\section{SDSS J222551.65+001637.7: A White Dwarf with a Partially Resolved Brown Dwarf Companion}

SDSS J2225+001 shows no sign of an M dwarf in it's optical spectrum and an atmospheric model (a cooler model was obtained in this case) with parameters of $T_{\text {eff }}=$ $10,000 \mathrm{~K}$ and $\log g=8.00$, a close matched to the EIS06 autofit parameters of $T_{\text {eff }}=$ 


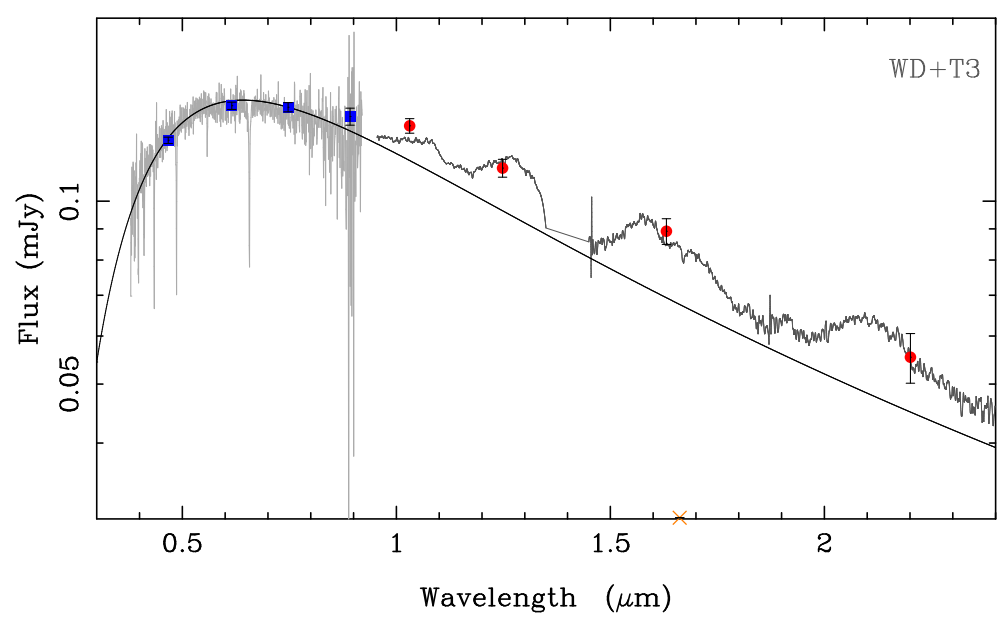

FIGURE 2.19. SDSS J154431.47+060104.3 model spectrum (solid black) with SDSS griz' (blue) and UKIDSS $Y J H K$ photometry (red). Also shown are the SDSS spectrum (light grey) and a composite WD+T3 dwarf spectrum (dark grey). 
$10640 \pm 94 \mathrm{~K}$ and $\log g=8.16 \pm 0.09$ ) matches the SDSS photometry (Figure 2.20). A clear excess is then seen in the $H$ and $K$-band photometry with the predicted absolute $K$-band magnitude of the secondary suggesting a spectral type of approximately L6. A composite $\mathrm{WD}+\mathrm{L} 7$ model provides the best match in this case. The UKIDSS mergedClass statistic for SDSS J2225-001 suggest the system is a partially resolved. However, there is not a clear separation of the components in the UKIDSS images. The measured FWHM of the star is 2.28 " in the $K$-band which equates to a projected orbital separation of $<435 \mathrm{AU}$ for the secondary at the estimated distance to the white dwarf (Table 2.9).

Since SDSS J2225+001 is a good candidate for a rare, previously unknown unresolved substellar companion to a white dwarf, independent $J H K$ photometry was obtained at the 3.5m NTT at La Silla in October 2007 (Section 2.4). Indeed, the SOFI $J H K$ photometry confirms the NIR excess and is a good fit to the WD+L7 composite model. The SOFI $K$-band image of SDSS J2225+001 is shown in Figure 2.21, and shows that the system may indeed become resolved with a higher resolution instrument. The FWHM of the star in this image is 1.8 " which equates to an orbital separation of $<350 \mathrm{AU}$.

\subsubsection{Magnetic White Dwarfs}

Twelve hydrogen atmosphere magnetic white dwarfs (DAHs) and a single helium atmosphere magnetic white dwarf $(\mathrm{DBH})$ were found in the cross correlation between the EIS06 and McCook \& Sion catalogues, and the UKIDSS DR5 database. All of these were found to fit their predicted near-infrared photometry to within $3 \sigma$ of their UKIDSS 


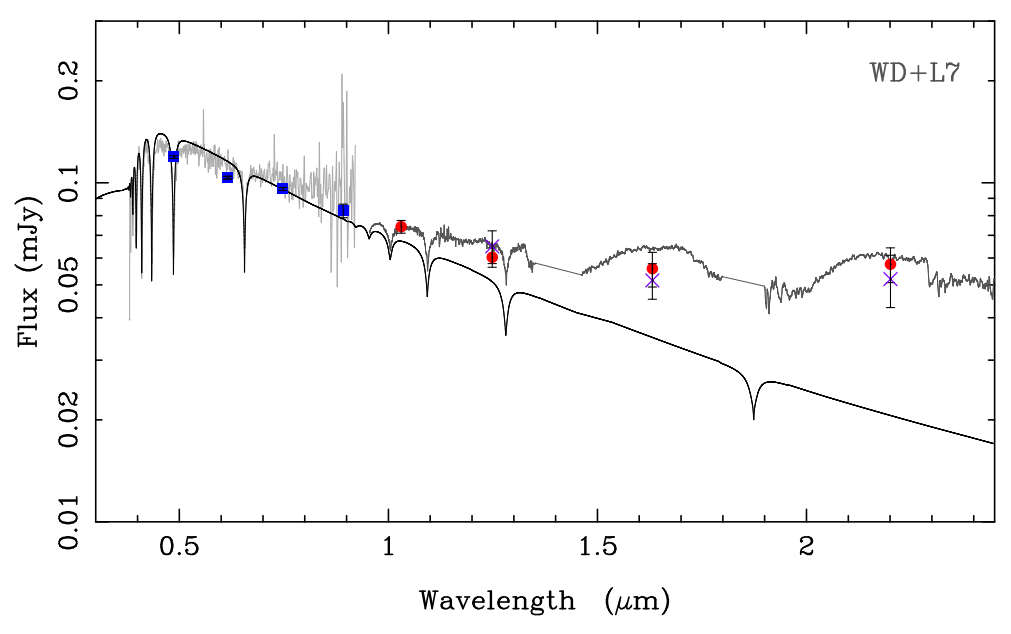

FIGURE 2.20. SDSS J222551.65+001637.7 model spectrum (solid black) with SDSS griz' (blue) and UKIDSS $Y J H K$ photometry (red). Also shown are the SDSS spectrum (light grey) and a composite WD+L7.5 dwarf spectrum (dark grey).

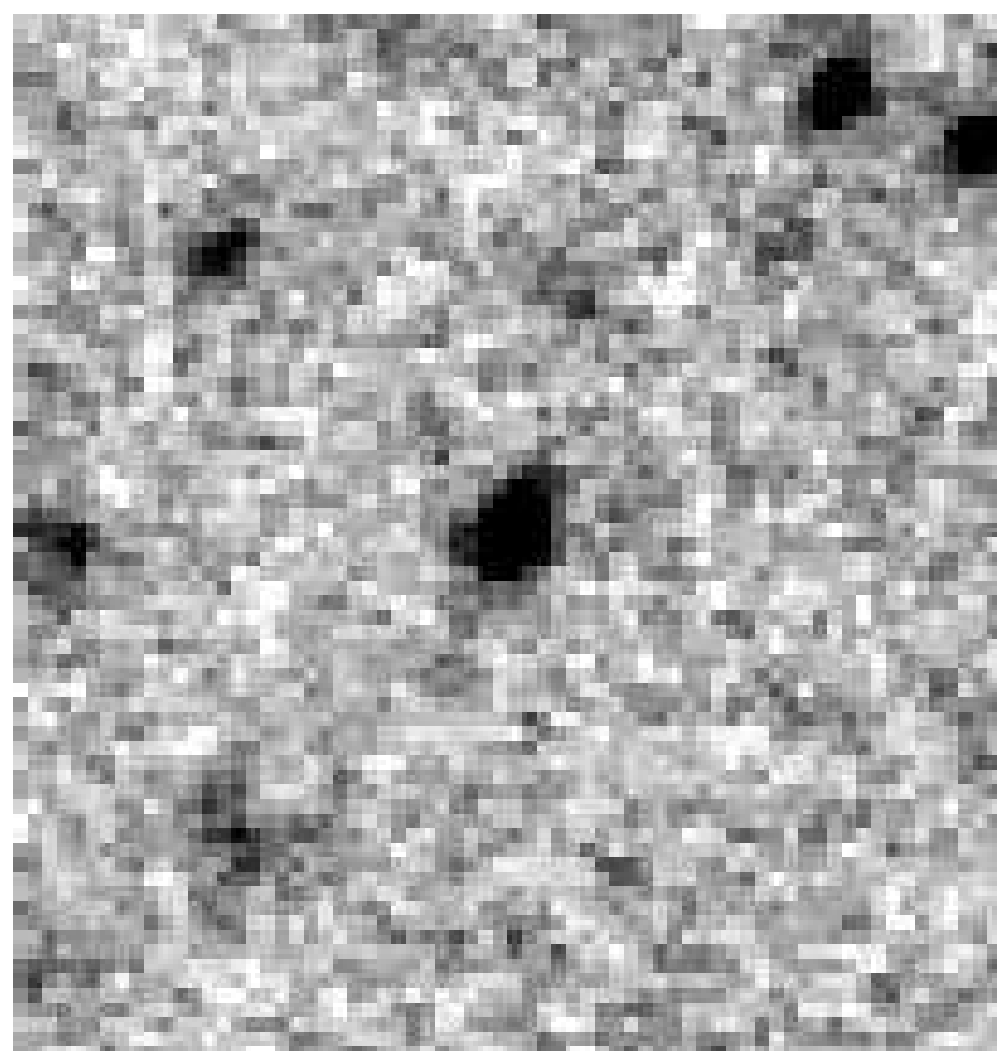

FIGURE 2.21. $K$-band image of SDSS J222551.65+001637.7 taken with SOFI on the NTT. The star is in the centre and is clearly elongated, possibly indicating the system is becoming resolved. 
observed values, with the exception of SDSS J121209.31+013627.7 (see below). In an attempt to increase this sample size, a further cross correlation was performed between the UKIDSS DR5 database and a list of known magnetic white dwarfs from Kawka et al. (2007). However, after accounting for duplicate entries between the catalogues, this only increased the sample by a single DAH star. Fortunately this star turned out to exhibit a near-infrared excess and is discussed below.

\section{SDSS J121209.31+013627.7}

SDSS J1212+013 was first identified as a binary through an optical spectrum and a single photometric $J$-band detection, limiting the spectral type of the secondary to L5 or later (Schmidt et al. 2005). It was initially though to be a detached system until Debes et al. (2006) detected strong cyclotron emission, an indication of mass transfer, in $K$-band time series photometry. Both Koen \& Maxted (2006) and Burleigh et al. (2006b) detected optical variability consistent with that of a polar in a low accretion state. The nearinfrared excess was successfully modelled by Farihi et al. (2008), who modelled the system as a DAH + a brown dwarf companion of spectral type L8. Although the evidence suggests that SDSS J1212+013 is a CV in a low state, the near-infrared observations do not rule out accretion occurring via a wind. Therefore, this system shall be considered for statistical analysis (Chapter 6). 
SDSS J125044.42 $+154957.4=$ WD $1248+161$

WD $1248+161$ is a DAH white dwarf identified by Vanlandingham et al. (2005) in the SDSS DR3. It has an estimated effective temperature of $T_{\text {eff }}=10,000 \mathrm{~K}$ and a polar magnetic field strength $B_{p}=20 \mathrm{MG}$. The SDSS optical spectrum and photometry are a reasonable fit when compared to a blackbody model generated at the effective temperature of the star, with a clear excess in all four of the UKIDSS bands (Figure 2.22). It was found that all of the UKIDSS data could not be matched consistently with any composite model, with a discrepancy existing between the $J H$ and the $K$-band photometry. This could be explained by the existence of strong cyclotron emission due to the magnetic field of the white dwarf, increasing the flux in the $K$-band (Section 1.1.3). Therefore, the predicted absolute magnitude of the secondary was calculated using the $H$-band, which suggests a spectral type of M8. A composite WD+M8 model matches the UKIDSS $J H$ photometry, however the $J$-bands data must be interpreted with caution as it was flagged as 'noise' in the UKIDSS archive. Another source of this excess $K$-band emission cannot be ruled out at this stage, such as the presence of a debris disk, and so near-infrared spectroscopy is required to identify the source of this flux.

The UKIDSS mergedClass statistic for WD J1248+161 indicates the system is a point source, with a measured FWHM of 1.57 " in the $H$-band. This equates to a projected orbital separation of $<245 \mathrm{AU}$ for the putative secondary at the estimated distance to the white dwarf (Table 2.9). 


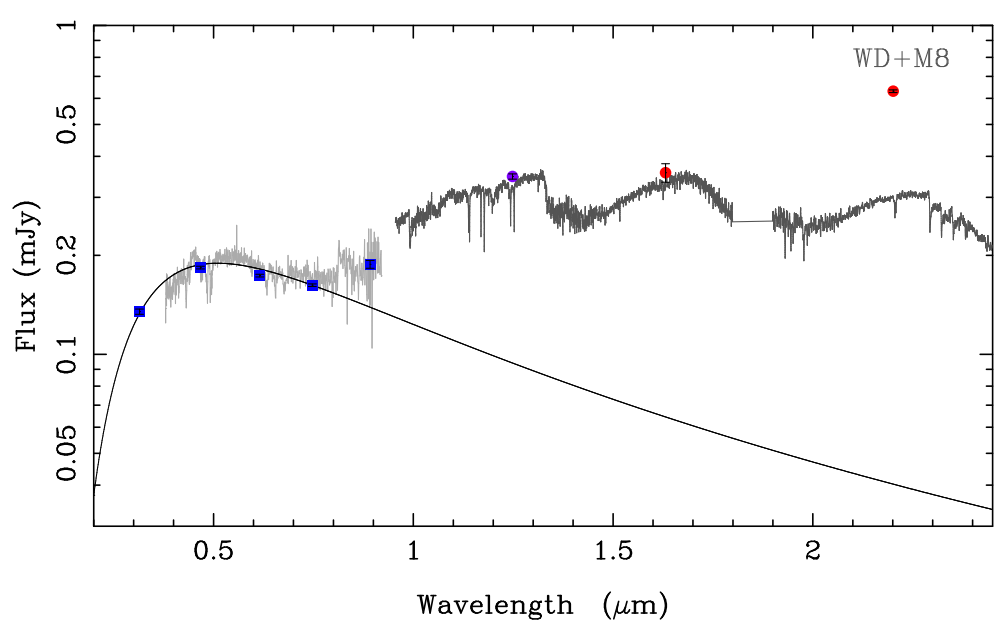

FIGURE 2.22. WD 1248+161 model spectrum (solid black) with SDSS griz' (blue) and UKIDSS $H K$ (red) photometry. Also shown are the SDSS spectrum (light grey) and a composite WD+M8 dwarf spectrum (dark grey). The single $J$-band observation (purple) was classified as noise in the DR5 database and should be treated with caution. 


\subsubsection{Debris Disks}

SDSS J122859.92+104033.0

SDSS J1228+104 was first identified by Gänsicke et al. (2006) in DR6 of the SDSS, where they identified distinct emission lines of the Calcium $850-866 \mathrm{~nm}$ triplet. The lines profiles of the Ca emission triplet show a double-peaked morphology, indicative of a gaseous, rotating disk. The velocity of the $\mathrm{Ca}$ II lines peaks indicated an outer radius of the disk of $\sim 1.2 R_{\odot}$. The star was later investigated in the NIR by Brinkworth et al. (2009), where it was discovered to have a large near- and mid-infrared excess, an indication of the presence of a dusty component in addition to that of the gas. This was the first evidence for the coexistence of a both a gaseous and dusty debris disk around a white dwarf.

SDSS J1228+104 was recovered in this work as having a large $K$-band excess, as was expected from this system.

SDSS J132044.68 $+001855.0=$ WD $1318+005$

The SDSS optical spectrum of WD 1318+005 shows no evidence for a companion, and the UKIDSS $J H$ photometry can be matched with a white dwarf model for $T_{\text {eff }}=$ 19,649K and $\log g=8.36$ (Figure 2.23). However, the UKIDSS $K$-band measurement is $3.1 \sigma$ in excess of this model, and can be matched by the addition of a $600 \mathrm{~K}$ blackbody to the white dwarf model. The UKIDSS mergedClass statistic for WD 1318+005 (-1) in- 


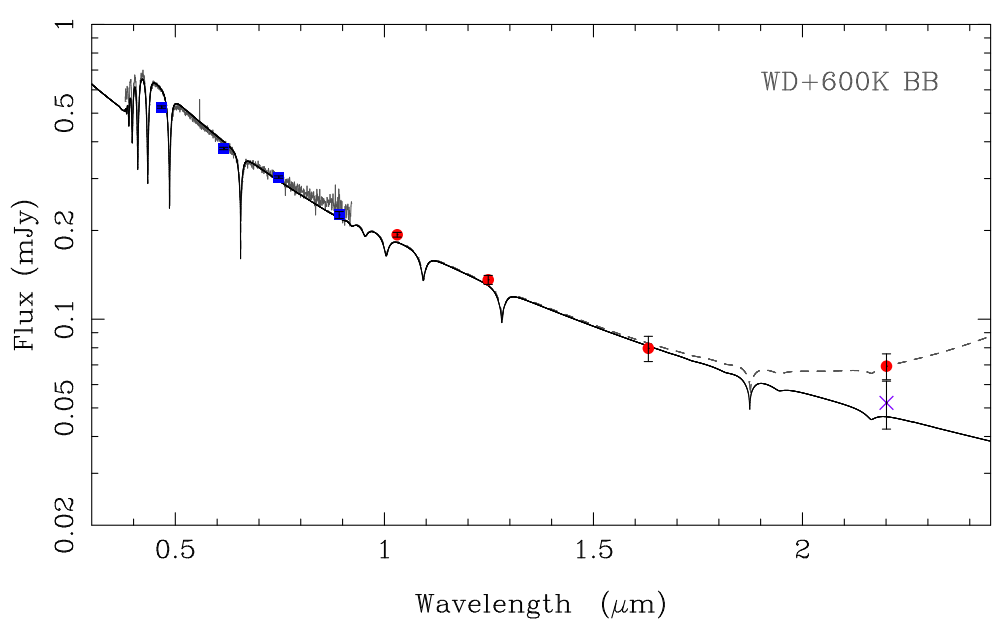

FIGURE 2.23. WD 1318+005 model spectrum (solid black) with SDSS griz' (blue), UKIDSS $Y J H K$ (red) and AAO IRIS2 $K$-band photometry (purple). Also shown are the SDSS spectrum (light grey) and a combined WD $+600 \mathrm{~K}$ blackbody model (dashed grey).

dicates the system is a point source, which rules out contamination due to a background galaxy.

Near-infrared photometry of WD $1318+005$ was also obtained with IRIS2 at the $3.9 \mathrm{~m}$ Anglo-Australian Telescope (Section 2.4). These data independently confirm the $K$ band excess emission (Figure 2.23). WD $1318+005$ possibly possesses a warm debris disk, and if so then it is highly likely to be a DAZ white dwarf. Although it cannot be classified as a DAZ from the existing low resolution SDSS optical spectrum, this star lies in the temperature regime of these objects.

\section{SDSS J155720.77+091624.7}

SDSS J1557+091 shows no optical excess when fitted with an atmospheric model with physical parameters of $T_{\text {eff }}=21990 \pm 403 \mathrm{~K}$ and $\log g=7.67 \pm 0.06$ (EIS06). An excess 


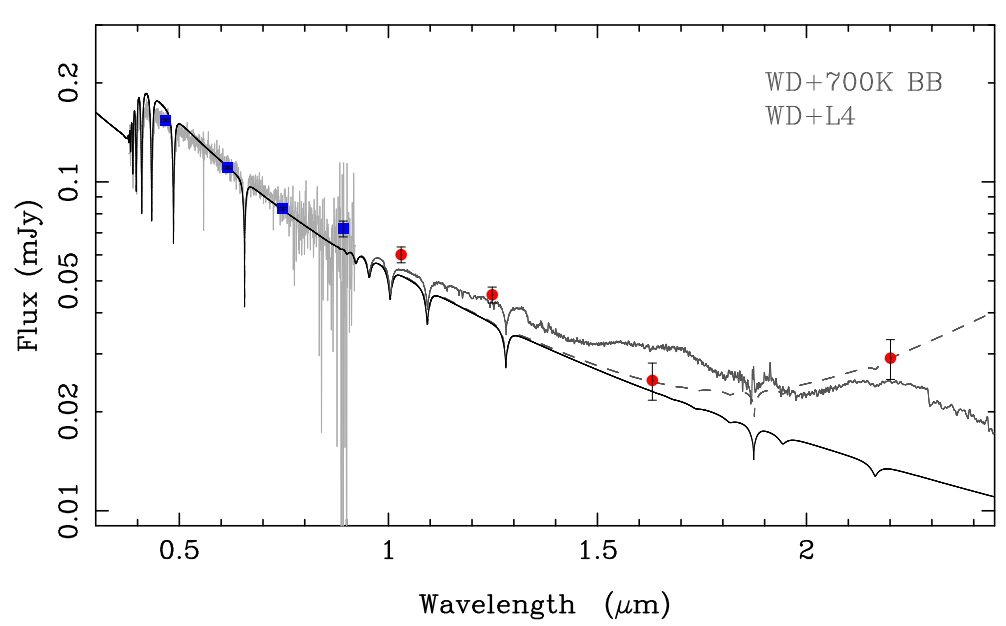

FIGURE 2.24. SDSS J155720.77+091624.7 model spectrum (solid black) with SDSS griz' (blue) and UKIDSS $Y J H K$ photometry (red). Also shown are the SDSS spectrum (light grey), a combined WD+L4 dwarf model (dark grey), and a combined WD $+700 \mathrm{~K}$ blackbody model (dashed grey).

is clearly evident in the $K$-band photometry which is best matched by the addition of a $700 \mathrm{~K}$ blackbody, indicating a potential cool disk. However, it should also be noted that there is a possible excess in the $Y$ and $J$-band photometry which can be best matched by the addition of a companion with spectral type L4. This is within $2 \sigma$ of the $H$-band flux and so a sub-stellar companion, or indeed another source, cannot be discounted as a possibility at this stage. The UKIDSS mergedClass statistic for SDSS J1557+091 indicates the system is a probable point source $(-2: 70 \%$ probability that the source is stellar), with a measured FWHM of 0.51 " in the $J$-band. This equates to a projected orbital separation of $<250 \mathrm{AU}$ for a secondary star at the estimated distance to the white dwarf (Table 2.9). 


\subsection{Low Mass Star or Brown Dwarf?}

In order to assess whether or not each putative companion is a low mass main-sequence star or a brown dwarf an estimate of the mass of the secondary has been calculated. Firstly an age of the white dwarf was calculated by the addition of the white dwarf cooling age (Section 2.3.3) and the main-sequence lifetime of the progenitor star. This was estimated using the initial-final mass relationship of Dobbie et al. (2006) (Section 1.1.5), which is valid for initial masses $>1.6 M_{\odot}$ (Kalirai et al. 2008). An approximate mainsequence lifetime can then be calculated from the models of Girardi et al. (2000). It should be noted that for white dwarfs where $M_{\mathrm{WD}}<0.5 M_{\odot}$ it is highly likely that the star has evolved through mass transfer and for these stars an age can not be calculated through this method. However, this can be seen as further evidence for the existence of a secondary star. For these stars, a lower limit on the mass of the secondary is estimated by using the cooling age of the white dwarf.

A mass for the secondary can then be estimated by interpolating the DUSTY atmospheric models (Chabrier et al. 2000, Baraffe et al. 2002), given the age of the white dwarf and an estimate of effective temperature of the companion. The temperatures were estimated by comparison with observed M, L and T-dwarfs (Vrba et al. 2004) and assuming an error of \pm 1 spectral type. For the WD $+\mathrm{dM}$ binaries, masses have been estimated by referring to the models of Baraffe \& Chabrier (1996). The results are given in Table 2.9 and plotted in Figure 2.25. 
Table 2.8. Summary of proposed sources for the near-infrared excesses identified in UKIDSS. For the resolved systems, the probability of a chance alignment is given. Further information on systems with a putative companion is given in Table 2.9.

\begin{tabular}{|c|c|c|c|}
\hline SDSS J & WD & Proposed Source of Excess & Figure \\
\hline "003902.47-003000.3 & - & "Unresolved Companion & 2.7 \\
\hline $003923.04+003534.7$ & - & Unresolved Companion & A. 1 \\
\hline $012032.27-001351.1$ & - & Resolved Companion $\left(\mathrm{P}_{\mathrm{al}}=5.8 \times 10^{-2}\right)$ & 2.8 \\
\hline $013532.98+144555.8$ & $0132+145$ & Unresolved Companion & 2.11 \\
\hline $023247.50-003909.3$ & - & Foreground/Background Object & 2.13 \\
\hline $032317.00-002612.7$ & - & Foreground/Background Object & A. 2 \\
\hline $034221.55+005345.6$ & - & Foreground/Background Object & A. 3 \\
\hline $085956.47+082607.5$ & - & Unresolved Companion & A. 4 \\
\hline $090759.59+053649.7$ & - & Unresolved Companion & A. 5 \\
\hline $092452.73+020712.2$ & $0922+006$ & Resolved Companion $\left(\mathrm{P}_{\mathrm{al}}=2.8 \times 10^{-4}\right)$ & 2.16 \\
\hline $092648.84+102828.8$ & - & Unresolved Companion & A. 6 \\
\hline $100259.88+093950.0$ & - & Unresolved Companion & A. 7 \\
\hline $101642.93+044317.7$ & - & Unresolved Companion & A. 8 \\
\hline $103220.19+011227.0$ & - & Foreground/Background Contamination & A.9 \\
\hline $103448.92+005201.4$ & $1032+011$ & Unresolved Companion & A. 10 \\
\hline $103736.75+013912.2$ & - & lved Companion & A.11 \\
\hline $104933.58+022451.7$ & - & Foreground/Background Object & A. 12 \\
\hline $105332.62+020126.2$ & - & Foreground/Background Object & A.13 \\
\hline $113416.09+055227.2$ & - & Unresolved Companion & A.14 \\
\hline $115808.44-012312.9$ & $1155-011$ & Unresolved Companion & A. 15 \\
\hline $121209.31+013627.7$ & - & Unresolved Companion (Magnet & - \\
\hline $125044.42+154957.4$ & $1248+161$ & Unresolved Companion (Magnetic WD) & 2.22 \\
\hline $122859.92+104033.0$ & - & Debris Disk & - \\
\hline $132044.68+001855.0$ & $1318+005$ & Debris Disk & 2.23 \\
\hline $132925.21+123025.4$ & - & Unresolved Companion & A.16 \\
\hline $154431.47+060104.3$ & - & Unresolved Companion & 2.19 \\
\hline $155720.77+091624.7$ & - & Debris Disk & 2.24 \\
\hline $220841.63-000514.5$ & - & Resolved Companion $\left(\mathrm{P}_{\mathrm{al}}=3.7 \times 10^{-4}\right)$ & A.17 \\
\hline $222030.68-004107.9$ & - & Partially Resolved Companion & - \\
\hline $222551.65+001637.7$ & - & Partially Resolved Companion & 2.20 \\
\hline $233345.97-000843.0$ & - & Unresolved Companion & A.19 \\
\hline
\end{tabular}


If a value of $70 M_{\mathrm{J}}$ is assumed as a conservative cut-off mass for the stellar-substellar boundary, then 6 new DA white dwarf + brown dwarf binaries and 1 new DB white dwarf + brown dwarf binaries have potentially been discovered (1 DA + L8 has been spectroscopically confirmed, Steele et al. 2009). All of these have spectral types of L4 or later, with one candidate WD + T-dwarf. 
Table 2.9. Table of results for SDSS white dwarfs with NIR excess indicative of a companion: most likely companion spectral types and mergedClass (MC) stats indicating the possibility that a system is a widely separated (+1) or a close detached binary ( -1 or -2$)$.

\begin{tabular}{|c|c|c|c|c|c|c|c|c|}
\hline SDSS J & WD & Spec. Types & $\mathrm{MC}$ & $\mathrm{M}_{\mathrm{WD}}\left(\mathrm{M}_{\mathrm{s}}\right)$ & $\mathrm{d}(\mathrm{pc})$ & Total Age (Gyr) & $\mathrm{M}_{\mathrm{COMP}}\left(\mathrm{M}_{\mathrm{Jup}}\right)$ & $\mathrm{a}(\mathrm{AU})$ \\
\hline $101642.93+044317.7$ & 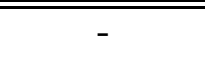 & $\overline{\mathrm{DA}+\mathrm{dM} 3}$ & -2 & $0.40 \pm 0.01$ & $6600 \pm 30$ & $>0.01$ & $350 \pm 150$ & $<<530$ \\
\hline $085956.47+082607.5$ & - & $\mathrm{DA}+\mathrm{dM} 6$ & -1 & $0.36 \pm 0.02$ & $400 \pm 20$ & $>0.1$ & $108 \pm 18$ & $<650$ \\
\hline $113416.09+055227.2$ & - & $\mathrm{DA}+\mathrm{dM} 6$ & -1 & $0.57 \pm 0.04$ & $580 \pm 50$ & $1.6_{-0.4}^{+0.7}$ & $108 \pm 18$ & $<510$ \\
\hline $012032.27-001351.1$ & - & $\mathrm{DA}+\mathrm{dM} 7$ & -1 & $0.56 \pm 0.12$ & $410 \pm 70$ & $2.2_{-1.1}^{+5.8}$ & $93 \pm 8$ & $\approx 910$ \\
\hline $103736.75+013912.2$ & - & $\mathrm{DA}+\mathrm{dM} 7$ & -1 & $0.49 \pm 0.02$ & $330 \pm 10$ & $3.7_{-0.8}^{+1.1}$ & $93 \pm 8$ & $<330$ \\
\hline $115808.44-012312.9$ & $1155-011$ & $\mathrm{DA}+\mathrm{dM} 7$ & -1 & $\approx 0.60^{1}$ & $280 \pm 10$ & $\approx 1.8^{1}$ & $93 \pm 8$ & $<270$ \\
\hline $132925.21+123025.4$ & - & $\mathrm{DA}+\mathrm{dM} 7$ & -1 & $0.36 \pm 0.01$ & $210 \pm 10$ & $>0.2$ & $93 \pm 8$ & $<235$ \\
\hline $233345.97-000843.0$ & - & $\mathrm{DA}+\mathrm{dM} 7$ & -1 & $0.83 \pm 0.18$ & $390 \pm 100$ & $1.1_{-0.4}^{+1.2}$ & $93 \pm 8$ & $<350$ \\
\hline $092648.84+102828.8$ & - & $\mathrm{DA}+\mathrm{dM} 8$ & -1 & $0.68 \pm 0.17$ & $480 \pm 110$ & $1.0_{-0.5}^{+2.3}$ & $85 \pm 5$ & $<800$ \\
\hline $125044.42+154957.4$ & $1248+161$ & $\mathrm{DAH}+\mathrm{dM} 8$ & -1 & $\approx 0.60^{1}$ & $150 \pm 10$ & $>0.6$ & $85 \pm 5$ & $<245$ \\
\hline $003923.04+003534.7$ & - & $\mathrm{DA}+\mathrm{dM} 9$ & -1 & $0.60 \pm 0.06$ & $230 \pm 20$ & $1.8_{-0.5}^{+0.9}$ & $80 \pm 3$ & $<263$ \\
\hline $003902.47-003000.3$ & - & $\mathrm{DA}+\mathrm{dLO}$ & -1 & $0.34 \pm 0.02$ & $390 \pm 30$ & $>0.3$ & $>80$ & $<484$ \\
\hline $100259.88+093950.0$ & - & $\mathrm{DA}+\mathrm{dLO}$ & -1 & $0.57 \pm 0.03$ & $540 \pm 30$ & $1.6_{-0.3}^{+0.5}$ & $78 \pm 7$ & $<420$ \\
\hline $220841.63-000514.5$ & - & $\mathrm{DA}+\mathrm{dL} 1$ & +1 & $0.78 \pm 0.08$ & $170 \pm 20$ & $1.5_{-0.3}^{+0.6}$ & $71 \pm 5$ & $\approx 290$ \\
\hline $090759.59+053649.7$ & - & $\mathrm{DA}+\mathrm{dL} 4$ & -1 & $0.54 \pm 0.02$ & $360 \pm 20$ & $2.1_{-0.4}^{+0.5}$ & $58 \pm 2$ & $<600$ \\
\hline $092452.73+020712.2$ & $0922+006$ & $\mathrm{DB}+\mathrm{dL} 4$ & +1 & $0.90 \pm 0.06$ & $200 \pm 20$ & $0.6_{-0.2}^{+0.4}$ & $55 \pm 6$ & $<\approx 240$ \\
\hline $013532.98+144555.8$ & $0132+145$ & $\mathrm{DA}+\mathrm{dL} 5$ & -1 & $0.65 \pm 0.03$ & $60 \pm 10$ & $2.1_{-0.2}^{+0.3}$ & $58 \pm 2$ & $<57$ \\
\hline $103448.92+005201.4$ & $1032+011$ & $\mathrm{DA}+\mathrm{dL} 5$ & -1 & $0.68 \pm 0.10$ & $200 \pm 30$ & $1.4_{-0.4}^{+0.9}$ & $55 \pm 4$ & $<150$ \\
\hline $222551.65+001637.7$ & - & $\mathrm{DA}+\mathrm{dL} 7$ & +1 & $0.70 \pm 0.06$ & $190 \pm 20$ & $1.2_{-0.3}^{+0.4}$ & $47 \pm 3$ & $<350$ \\
\hline $121209.31+013627.7$ & - & $\mathrm{DAH}+\mathrm{dL} 8$ & -1 & $\approx 0.60^{2}$ & $\approx 150^{2}$ & $1-5^{2}$ & $\approx 50^{2}$ & $\approx 0.003$ \\
\hline $222030.68-004107.9$ & - & $\mathrm{DA}+\mathrm{dL} 8$ & +1 & $0.77 \pm 0.04$ & $60 \pm 10$ & $2.1_{-0.3}^{+0.4}$ & $49 \pm 3$ & 55 \\
\hline $154431.47+060104.3$ & - & $\mathrm{DA}+\mathrm{dT} 3$ & -1 & $0.85 \pm 0.09$ & $90 \pm 10$ & $2.6_{-0.8}^{+0.8}$ & $46 \pm 2$ & $<190$ \\
\hline
\end{tabular}

${ }^{1}$ Surface gravity estimated from mass distribution of white dwarfs (Typical white dwarf mass assumed).

${ }^{2}$ Farihi et al. (2008) 


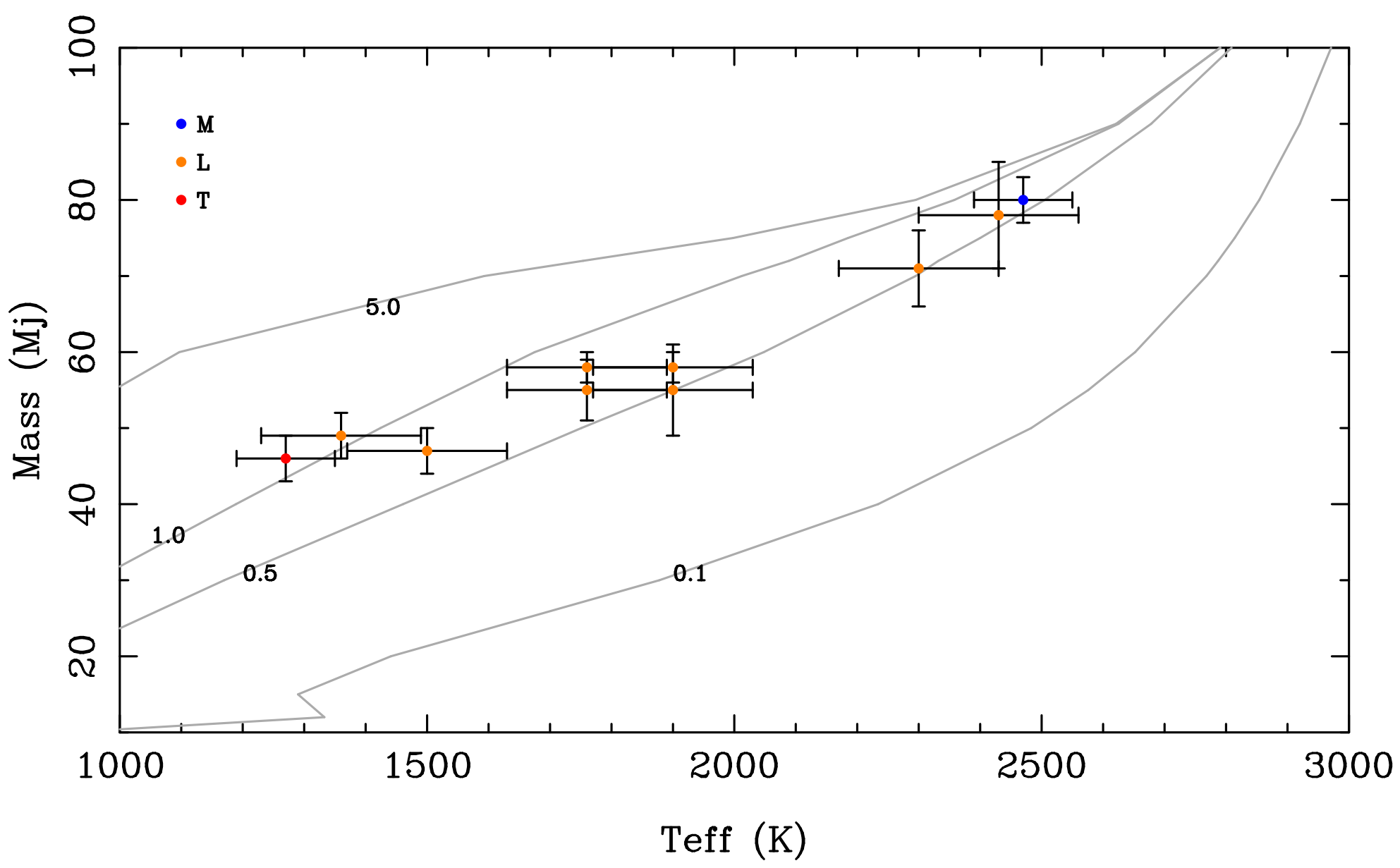

FIGURE 2.25. Predicted masses of companions based on the DUSTY models. The solid grey lines show the DUSTY models of various ages in Gyrs. 


\subsection{A Wide or Close Binary?}

The evidence thus far suggests a bimodal distribution of separation for white dwarf + low mass secondary binaries (Section 1.2). The UKIDSS mergedClass statistic may provide us with evidence which supports this conclusion. The white dwarf PHL 5038 (Chapter 5) was identified using this statistic as a partially resolved source, and was later fully resolved into a wide orbiting $(\approx 55 \mathrm{AU}) \mathrm{WD}+\mathrm{L} 8$ binary. Therefore, it may be possible to separate these stars into long and short period systems using this value.

In order to investigate this possibility, the resolving power of UKIDSS must be determined for the binary candidates in this survey. The UKIDSS photometry is measured using a 2" aperture in all bands. The projected orbital separation for this radius at distances from $10-100 \mathrm{pc}$ are plotted in Figure 2.26. The same is also plotted for the average FWHM of the binary candidates (1.2"). It can be seen that for the majority of stars it will not be possible to distinguish between long (Orbital distance $>$ few AU) and short period (Orbital distance $<0.1 \mathrm{AU}$ ) period systems if the system is classified as a point source, unless the binary has a particularly wide orbit. The maximum and projected orbital separations for all unresolved and resolved candidates in this survey are given in Table 2.9.

\subsection{Summary}

The UKIDSS LAS DR5 has been cross correlated with both the Eisenstein et al. (2006) and the McCook \& Sion online catalogues of spectroscopically identified white dwarfs, 


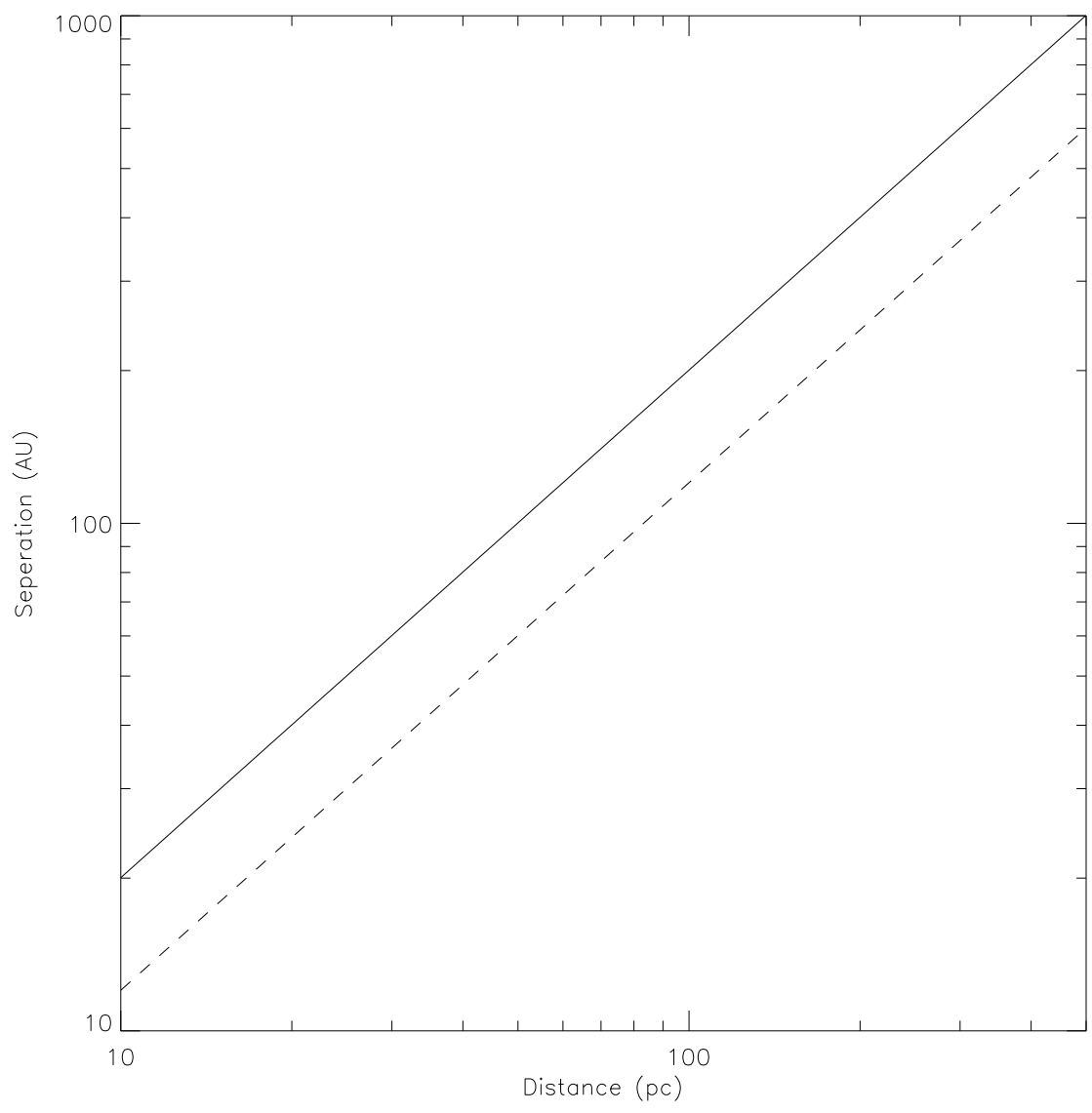

FIGURE 2.26. Distance verses projected orbital separation for a 2" (solid) and 1.2" (dashed) radius. 
in order to search for stars displaying NIR excesses indicative of low mass companions or debris disks. The sample included 639 hydrogen atmosphere DA white dwarfs with $1500<T_{\text {eff }}<100,000 \mathrm{~K}$ and $6.5<\log g<9.5$, and 10 helium atmosphere DB white dwarfs with $3500<T_{\text {eff }}<30,000 \mathrm{~K}$ and $7.0<\log g<9.0$, all with both $H$ and $K$-band photometry present in the UKIDSS archive.

235 white dwarfs were identified with NIR excesses, with 206 of these recovering previously identified DA $+\mathrm{M}$ and $\mathrm{DB}+\mathrm{M}$ type systems. This left 29 candidate white dwarfs for further analysis. 24 of these had multiple excesses indicative of a low mass companion, with 7 of these having a predicted mass in the range associated with brown dwarfs, and a further 13 likely very low mass stellar companions. Two white dwarfs were identified with putative companions that are likely spectral class K. Four of the sample showed evidence of contamination by a foreground or background object. The remaining three each had a $K$-band excess indicative of a debris disk including the already discovered DAd SDSS J1228+104. Two magnetic white dwarfs were found with a NIR excess; (1) SDSS J1212+01 - a previousley identified DAH +dL8 binary, and (2) SDSS J1250+154 - potentially explained by the presence of an M8 companion and additional cyclotron emission. 


\section{Chapter 3}

\section{NIR Spectroscopic Observations of WD + dM Binaries}

\subsection{Overview}

M-dwarfs are typically assigned spectral types based on key features in their optical spectra. An M-dwarf is formally identified by the onset of titanium oxide absorption (Morgan et al. 1943) in the blue/green end of the optical spectrum (although this can already be strong at infrared wavelengths). Titanium oxide absorption peaks at around M5, with vanadium oxide absorption dominating in the later spectral types. However, as M-dwarfs are low luminosity red stars it seems that a classification system based on redder wavelengths would be more appropriate. To that end, the system "officially" used today is that of Kirkpatrick, Henry \& McCarthy (1991). Their system is based on a point-by-point least squares comparison of low resolution spectra across the wavelength range $0.63-0.9 \mu \mathrm{m}$ against a grid of standard stars, comparing both the relative strength of key absorption lines ( $\mathrm{TiO}, \mathrm{VO}$ and $\mathrm{CaH})$ and the overall shape of the spectrum. 
White dwarf + M-dwarf binaries are usually dominated by the flux of the latter at the red end of an optical spectrum, with the white dwarf practically drowned out at near-infrared wavelengths. Thus, the two components can easily be separated using broadband nearinfrared photometry and spectroscopy. There are many examples of near-infrared photometric searches for white dwarfs with red companions (Green et al. 2000b, Wachter et al. 2003, Farihi, Becklin \& Zuckerman 2006, Debes et al. 2007, Mullally et al. 2007). These can then assigned spectral types by measuring the excess of flux above the continuum level of the white dwarf primary and comparing to existing data and/or models. However, irradiation by a close white dwarf companion, or activity in the secondary dwarf star, can lead to an increase in the excess emission observed in the infrared, and a change in the strength of the absorption lines of the companion. For example, K I (at $1.25 \mu \mathrm{m}$ ) and $\mathrm{Na} \mathrm{I}$ (at $2.2 \mu \mathrm{m}$ ) absorption are strengthened with the onset of activity in mid to late-type M-dwarfs (Kafka \& Honeycutt 2006). Therefore, the most accurate method is to spectrally type the secondary based on key absorption line strengths in conjunction with measuring the excess flux as outlined above.

Near-infrared spectroscopy also allows for the investigation of post-common envelope (CE) binaries (pre-CVs: Schreiber \& Gänsicke 2003). Tappert et al. (2007) analysed $K$-band spectroscopy of a sample of pre-CVs in order to investigate the strength of the $\mathrm{CO}$ features. Any anomalous abundances in this feature indicate that the secondary star has CNO processed material which has found its way into the photosphere. There are two possible explanations; (1) The nuclear evolution of the secondary star off the main sequence can account for this material. However, as the stars studied by Tappert et al. 
(2007) are of comparatively late spectral types $(\geq \mathrm{K} 0)$ this is ruled out as a scenario for these anomalies, as insufficient time for nuclear evolution would have passed before the $\mathrm{CE}$ to $\mathrm{CV}$ phase i.e. the secondary will remain in its hydrogen burning phase throughout the evolution of the white dwarf progenitor. (2) Accretion of the CNO material by the secondary star during the CE phase or through the ejection of material in nova eruptions. Therefore, anomalous $\mathrm{CO}$ abundances in these low mass companions would indicate material has been acquired during the $\mathrm{CE}$ phase. A number of the secondaries in this work are probably in close orbits with the primary and so a similar analysis to that of Tappert et al. can be carried out.

This chapter presents a detailed near-infrared spectroscopic study of eleven DA white dwarfs looking at binary systems with confirmed low mass M-type companions. Nine of the sample are hot $\left(T_{\text {eff }}>18,000 \mathrm{~K}\right)$ white dwarfs which all have near-infrared spectra consistent with early to mid-M type companions. I have also looked at two cooler DAs $\left(T_{\text {eff }}<9000 \mathrm{~K}\right)$, one of which harbours a mid-M type and the other a late-M type companion (M8). A spectral type is assigned to each companion based on flux level and relative strength of key absorption lines in the near-infrared when compared to composite white dwarf + M-type spectra. These are compared to previous optical photometric estimates of the secondary's spectral type with near-infrared spectroscopic determinations. Seven of the secondary stars have spectral types in agreement with previous optical photometric estimates, with the remainder no more than \pm 1 spectral type away. I have searched for evidence of accretion by the secondary during a common envelope phase by looking for anomalous $\mathrm{CO}$ features in the spectra of the short period binaries. No such evidence was 
found.

\subsection{Observations}

Low resolution near-infrared spectra were obtained for six DA white dwarf + M-dwarf $(\mathrm{WD}+\mathrm{M})$ binaries using the ESO Son-of-Isaac (SOFI) infrared instrument on July $29^{\text {th }}$ to August $1^{\text {st }} 2004$, and August $22^{\text {nd }}$ 2004. SOFI operates at the Naysmyth A focus of the NTT and includes a Rockwell Hg:Cd:Te Hawaii detector with $1024 \times 102418.5 \mu \mathrm{m}$ pixels. In the low-resolution spectroscopic mode $(\lambda / \delta \lambda \sim 950$ with the 0.6 arcsec slit), as used for this work, coverage of the wavelength ranges $0.95-1.64 \mu \mathrm{m}$ and $1.53-2.52 \mu \mathrm{m}$ is provided by the 'blue' and 'red' grism, respectively.

Near-infrared spectra were also obtained for a further four DA white dwarf + M-dwarf binaries using the UKIRT CGS4 instrument on August $10^{\text {th }}$ and $12^{\text {th }} 2001$, and April $15^{\text {th }}$ and $26^{\text {th }} 2003$. The UIST instrument was also used for three of these on March $22^{\text {nd }}$, $23^{\text {rd }}$, and $25^{\text {th }} 2003$. CGS4 is $1-5 \mu \mathrm{m} 2 \mathrm{D}$ grating spectrometer containing a $256 \times 256$ InSb array installed in a cryostat. The observations used the $401 / \mathrm{mm}$ grating providing a wavelength coverage of $0.9-1.35 \mu \mathrm{m}$ which provides resolving powers of $500-220$, achieved with $300 \mathrm{~mm}$ focal length camera optics and a one-pixel-wide slit. UIST is a $1-5 \mu \mathrm{m}$ image spectrometer with a $1024 \times 1024 \mathrm{InSb}$ array. In spectroscopic mode it uses a $0.12^{\prime \prime} /$ pixel camera and for the observations the $H K$ grism was used covering a wavelength range of $1.6-2.2 \mu \mathrm{m}$. 
The observations were undertaken using the standard technique of nodding the point source targets back and forth along the spectrograph slit in an ABBA pattern. To facilitate the removal of telluric features from the target spectra and to provide an approximate flux calibration, a standard star was observed either immediately before or after each science integration. These were carefully chosen to lie within $\sim 0.1$ airmasses of each white dwarf. In addition, regular exposures of the arc lamps were obtained to permit reliable wavelength calibration of the spectra. All of the observations are summarised in Table 3.1.

\subsection{Data Reduction}

To reduce the SOFI data standard techniques were applied using software routines in the STARLINK packages KAPPA and FIGARO. In brief, a bad pixel mask was constructed by merging a list of anomalously valued pixels clipped from dark frames with a generic map of bad array elements obtained from the ESO SOFI web pages. ${ }^{1}$ This was applied to all the data. The science, standard star and arc lamp spectral images were flat fielded with a normalised response map appropriate to either the blue or the red grism setup. Subsequently, difference pairs were assembled from the science and standard star images and any significant remaining sky background removed by subtracting linear functions, fitted in the spatial direction, from the data. The spectra of the white dwarfs and the standard stars were then extracted and assigned the wavelength solution derived from the relevant arc spectrum. Any features intrinsic to the energy distributions

\footnotetext{
${ }^{1}$ www.ls.eso.org/lasilla/sciops/ntt/sofi/index.html
} 
Table 3.1. Summary table of observations for each white dwarf in the sample.

\begin{tabular}{llcccc}
\hline WD & Date & Instrument/Filter & Exposure Time (s) & No. Exposures & Standard Star \\
\hline \hline $0017+061$ & $30-07-2004$ & SOFI GBF & 60.0 & 8 & HIP 682 \\
$0017+061$ & $30-07-2004$ & SOFI GRF & 60.0 & 8 & HIP 682 \\
$0131-163$ & $10-08-2001$ & CGS4 $J$ & 30.0 & 8 & BS 695 \\
$0131-163$ & $10-08-2001$ & CGS4 $K$ & 30.0 & 4 & BS 695 \\
$0419-487$ & $22-08-2004$ & SOFI GBF & 30.0 & 4 & HIP 20654 \\
$0419-487$ & $22-08-2004$ & SOFI GRF & 30.0 & 8 & HIP 20654 \\
$0752-146$ & $23-03-2003$ & UIST HK & 240.0 & 8 & HIP 39187 \\
$1305+018$ & $31-07-2004$ & SOFI GBF & 60.0 & 6 & HIP 63683 \\
$1305+018$ & $31-07-2004$ & SOFI GRF & 60.0 & 6 & HIP 63683 \\
$1412-049$ & $29-07-2004$ & SOFI GBF & 60.0 & 8 & HIP 69747 \\
$1412-049$ & $30-07-2004$ & SOFI GRF & 60.0 & 8 & HIP 69747 \\
$1415+132$ & $15-04-2003$ & CGS4 $J$ & 60.0 & 12 & HIP 68868 \\
$1415+132$ & $22-03-2003$ & UIST H $K$ & 240.0 & 4 & HIP 68868 \\
$1622+323$ & $26-04-2003$ & CGS4 $J$ & 60.0 & 8 & HIP 80460 \\
$1622+323$ & $25-03-2003$ & UIST H $K$ & 240.0 & 4 & HIP 82372 \\
$1643+143$ & $31-07-2004$ & SOFI GBF & 60.0 & 4 & HIP 82372 \\
$1643+143$ & $31-07-2004$ & SOFI GRF & 60.0 & 4 & BS 6866 \\
$1845+019$ & $12-08-2001$ & CGS4 $J$ & 30.0 & 4 & BS 6866 \\
$1845+019$ & $12-08-2001$ & CGS4 $K$ & 30.0 & 4 & HIP 107333 \\
$2151-015$ & $01-08-2004$ & SOFI GBF & 60.0 & 6 & HIP 107333 \\
$2151-015$ & $01-08-2004$ & SOFI GRF & 60.0 & &
\end{tabular}


of the standard stars were identified by reference to a near-IR spectral atlas of fundamental MK standards (Wallace et al. 2000, Meyer et al. 1998, Wallace \& Hinkle 1997) and were removed by linearly interpolating over them. The spectrum of each white dwarf was then co-aligned with the spectrum of its standard star by cross-correlating the telluric features present in the data. The science spectra were divided by the standard star spectra and multiplied by a blackbody with the standard star $T_{\text {eff }}$, taking into account the differences in exposure times. Finally, the flux levels were scaled to (1) achieve the best possible agreement between the blue and the red spectrum of each white dwarf in the overlap region between $1.53-1.64 \mu \mathrm{m}$ and (2) obtain the best possible agreement between the spectral data and the $J, H$ and $K_{\mathrm{S}}$ photometric fluxes for each object (Table 3.4.2) derived from the 2MASS All Sky Data Release Point Source Catalogue magnitudes (Skrutskie et al. 1995) where zero magnitude fluxes were taken from Holberg \& Bergeron (2006).

CGS4 and UIST spectra were reduced using the latest version of the ORAC data reduction pipeline ${ }^{2}$. However, wavelength and flux calibration were applied manually as it was found that ORAC-DR did not do a satisfactory job of removing telluric features from the standard star spectra. In brief, ORAC-DR was used to conduct bad-pixel correction, flat fielding and the co-addition of the nodded images. The extraction and calibration of the spectrum was then continued as outlined previously using the KAPPA and FIGARO packages.

\footnotetext{
${ }^{2}$ http://www.oracdr.org/
} 


\subsection{Analysis of Data}

\subsubsection{White Dwarf Model Spectra}

For each object with $\mathrm{T}_{\text {eff }} \geq 16000 \mathrm{~K}$ we have generated a pure-H synthetic white dwarf spectrum (as outlined in Section 2.3.3) at the effective temperature and surface gravity given in Table 3.4.2. Model spectra were not calculated for the coolest two of the white dwarf sample (WD0419-487 and WD2151+015), as these stars are in the temperature range for which convection becomes a significant factor, and the TLUSTY models have problems converging. For these stars blackbody models were generated instead using the FIGARO routine bbody. The synthetic fluxes were normalised to the $V$-band magnitude of the relevant white dwarf (Table 3.2)

\subsubsection{Spectrally Typing Low Mass Stellar Companions}

Each object shows significant differences in the overall shape or level between the observed and synthetic white dwarf spectra which is consistent with the presence of a cool low mass stellar companion. Additionally, specific features in each spectrum typical of the energy distributions of $\mathrm{M}$ dwarfs were observed e.g. $\mathrm{K} \mathrm{I}$ and $\mathrm{Na} \mathrm{I}$ absorption at $1.25 \mu \mathrm{m}$ and $2.20 \mu \mathrm{m}$ respectively, $\mathrm{CO}$ at $1.6 \mu \mathrm{m}$ and $2.3 \mu \mathrm{m}$ respectively and $\mathrm{H}_{2} \mathrm{O}$ centred on 1.4 and $1.9 \mu \mathrm{m}$. Empirical models have been added of low-mass stellar objects to the white dwarf synthetic spectrum and these composites have been compared to the nearinfrared data. These templates have been constructed using the near-infrared spectra of 
Table 3.2. Summary of the physical parameters for each white dwarf used in this work. The first reference refers to the temperature and surface gravity, the second to the $V$-band magnitude, and the third to the distance. In cases where no reference has been provided, the distance has been derived by parallax measurements or from the Bloecker evolutionary models.

\begin{tabular}{llllllcc}
\hline WD & Other Names & $\mathrm{T}_{\text {eff }}(\mathrm{K})$ & $\log \mathrm{g}$ & $V$ & $\mathrm{D}(\mathrm{pc})$ & Separation $(\mathrm{AU})$ & Refs. \\
\hline \hline $0017+061$ & PG 0017+061 & 28273 & 7.756 & 14.75 & 102 & 266 & $1,2,2$ \\
$0131-163$ & GD 984, REJ 0134-160 & 43722 & 7.7 & 13.98 & 120 & 21 & $3,4,5$ \\
$0419-487$ & V* RR Cae, LTT 1951 & 7005 & 7.723 & 14.36 & 21 & - & 1,4 \\
$0752-146$ & LTT 2980 & 18600 & 7.7 & 13.59 & 35 & $<0.1$ & 5,6 \\
$1305+018$ & PG 1305+018 & 29430 & 7.82 & 15.16 & 48 & - & 7,7 \\
$1412-049$ & PG 1414-049 & 40000 & 7.8 & 16.74 & 333 & 1200 & $8,8,8$ \\
$1415+132$ & PG 1415+132, Feige 93 & 34004 & 7.427 & 15.29 & 210 & - & 1,4 \\
$1622+323$ & PG 1622+323 & 77166 & 7.838 & 16.33 & 520 & 49 & 1,9 \\
$1643+143$ & PG 1643+143 & 25455 & 7.791 & 15.38 & 150 & $<75$ & $1,4,5$ \\
$1845+019$ & REJ 1847+015, Lanning 18 & 29456 & 7.844 & 12.96 & 42 & - & $1,4,1$ \\
$2151+015$ & LTT 8747 & 8500 & 8.0 & 14.41 & 21 & 23 & $8,8,8$ \\
\hline
\end{tabular}

1. Finley (1997) 2. Liebert et al. (2005) 3. Green et al. (2000b) 4. McCook \& Sion (1999) 5. Farihi et al. (2005) 6. Guseinov et al. (1983)

7. Bergeron et al. (1992) 8. Farihi, Hoard \& Wachter (2006b) 9. Green et al. (1986) 
M-dwarfs from the IRTF spectral library (Cushing et al. 2005). The data have been flux calibrated using $J, H$ and $K_{\mathrm{S}}$ photometric fluxes derived from the 2MASS magnitudes (Table 3.3) as described by McLean et al. (2001).

The fluxes of the empirical templates have been scaled to a level appropriate to a location at $\mathrm{d}=10 \mathrm{pc}$ using distances estimated from the parallax of each object (Patten et al. 2006). Subsequently, these fluxes have been re-calibrated to be consistent with the distance of each white dwarf. Starting with a spectral type of MOV, later spectral types have been progressively added to the synthetic white dwarf spectrum, until it could be concluded with reasonable certainty that the correct flux level had been obtained.

For each binary system the synthetic white dwarf spectrum has been subtracted from the observed $J H K$ spectrum ( $J$ and $K$ only for CGS4 spectra). This gives an accurate representation of the flux level and spectral features of the companion. The IRTF spectrum of a star with the same estimated spectral type of the secondary was added to these plots to aid visual comparisons and help facilitate spectral typing. The existence of certain absorption lines and their relative strengths, e.g. K I and $\mathrm{Na}$ I, provide an additional constraint on the spectral type of the companion, which can be compared to those already ascertained through optical and near-infrared photometry.

Each star's spectrum was visually inspected and a qualitative comparison made to the scaled IRTF spectrum (as was the case in Tappert et al. 2007). In order to quantify the estimates of each of the secondary spectral types, a suitable absorption line was chosen to be measured. For the stars in this sample, the only common absorption line that can be 
Table 3.3. Summary details of the white dwarfs studied in this work, including near-IR magnitudes for each star obtained from the 2MASS All-Sky Point Source Catalogue.

\begin{tabular}{l|c|c|ccc}
\hline Identity & RA & Dec & $J$ & $H$ & $K_{\mathrm{S}}$ \\
& \multicolumn{2}{|c|}{ J2000.0 } & & & \\
\hline \hline WD 0017+061 & 001941.00 & +062407.6 & $13.738 \pm 0.033$ & $13.189 \pm 0.033$ & $12.983 \pm 0.032$ \\
WD 0131-163 & 013424.07 & -160708.1 & $12.966 \pm 0.027$ & $12.468 \pm 0.028$ & $12.215 \pm 0.030$ \\
WD 0419-487 & 042105.56 & -483907.0 & $10.720 \pm 0.024$ & $10.148 \pm 0.023$ & $9.852 \pm 0.025$ \\
WD 0752-146 & 075508.95 & -144551.0 & $12.621 \pm 0.024$ & $12.141 \pm 0.023$ & $11.837 \pm 0.019$ \\
WD 1305+018 & 130754.73 & +013210.7 & $12.982 \pm 0.026$ & $12.400 \pm 0.023$ & $12.144 \pm 0.021$ \\
WD 1412-049 & 141502.21 & -051104.0 & $13.803 \pm 0.030$ & $13.092 \pm 0.027$ & $12.988 \pm 0.034$ \\
WD 1415+132 & 141740.21 & +130148.6 & $14.263 \pm 0.037$ & $13.725 \pm 0.046$ & $13.553 \pm 0.046$ \\
WD 1622+323 & 162448.99 & +321702.2 & $14.633 \pm 0.029$ & $13.963 \pm 0.031$ & $13.773 \pm 0.039$ \\
WD 1643+143 & 164539.14 & +141746.2 & $12.732 \pm 0.024$ & $12.125 \pm 0.031$ & $11.957 \pm 0.024$ \\
WD 1845+019 & 184737.51 & +015724.7 & $15.861 \pm 0.086$ & $15.179 \pm 0.099$ & $14.692 \pm 0.111$ \\
WD 2151-015 & 215406.45 & -011710.3 & $12.452 \pm 0.029$ & $11.778 \pm 0.022$ & $11.414 \pm 0.027$ \\
\hline
\end{tabular}


distinguished from noise is $\mathrm{Na}$ I at $2.2 \mu \mathrm{m}$. This is ideal for spectral typing as it shows a distinct temperature dependence whilst being independent of luminosity class, and thus nuclear evolution (Ivanov et al. 2004). The results are discussed in Section 3.5.2.

\subsection{Results}

For the sake of brevity, only two examples are discussed in the following section. The remaining discussions and spectra are given in Appendix $B$. The results are summarised in Table 3.4.

\subsubsection{Examples of the Spectroscopic Classification of a Companion}

WD 0017 $+061=$ PHL 790

Farihi et al. (2005) used photometry to estimate the spectral type of the companion to PHL 790 as an M4 star at a projected orbital distance of 266 AU. Figure 3.1 shows the observed and modelled spectrum of PHL 790 and composite WD+M4 and WD+M3.5 models. Figures 3.2 and 3.3 show the model subtracted spectrum with an M4 star for comparison. The prominent $\mathrm{Na}$ I line at $1.14 \mu \mathrm{m}$ and the $\mathrm{H}_{2} \mathrm{O}$ centred on $1.4 \mu \mathrm{m}$ and $1.9 \mu \mathrm{m}$ are consistent with a young early M-type companion. Although the $K$-band becomes noisy towards $2.4 \mu \mathrm{m}$, the $\mathrm{CO}$ bands appear visually to be of the same strength as the M4 comparison spectrum. There is a weak Na I line at $2.2 \mu \mathrm{m}(\mathrm{EW}=3.9 \pm 0.9 \AA$, Table 3.4) which is also consistent with an M4 companion. The observed spectrum ap- 
pears at roughly the same flux level as a $\mathrm{WD}+\mathrm{M} 4$ model and so evidence agrees with the previous spectral typing. At an orbital distance of $266 \mathrm{AU}$, this system is not a candidate pre-CV.

\section{WD 0131-163 = GD 984}

Farihi et al. (2005) used photometry to estimate the spectral typed of the companion to GD 984 as an M2 orbiting at a projected distance of $<60$ AU. Shortly afterwards the system was resolved with the Hubble Space Telescope by Farihi, Hoard \& Wachter (2006b) who then estimated the spectral type of the companion, again from photometry, as an M3.5 orbiting at a projected distance of 21 AU. Figure 3.4 shows the observed $J$ and $K$ and modelled spectra of GD 984 and composite WD+M3, WD+M3.5 and WD+M4 models. Figures 3.5 and 3.6 show the model subtracted spectrum with an M3.5 for comparison. The strong Na I emission line at $2.2 \mu \mathrm{m}(\mathrm{EW}=7.5 \pm 0.6 \AA$, Table 3.4$)$ suggests an early $\mathrm{M}$ type companion and is consistent with the template M3.5 spectrum, an estimate strongly backed by the presence of the visually strong $\mathrm{Na}$ I line at $1.14 \mu \mathrm{m}$ and the close correlation to the flux level of an M3.5 companion at $120 \mathrm{pc}$. The CO bands at $2.3 \mu \mathrm{m}$ do not appear to have been detected at all in the spectrum, probably due to insufficient $\mathrm{S} / \mathrm{N}$ at wavelengths $>2.3 \mu \mathrm{m}$. 


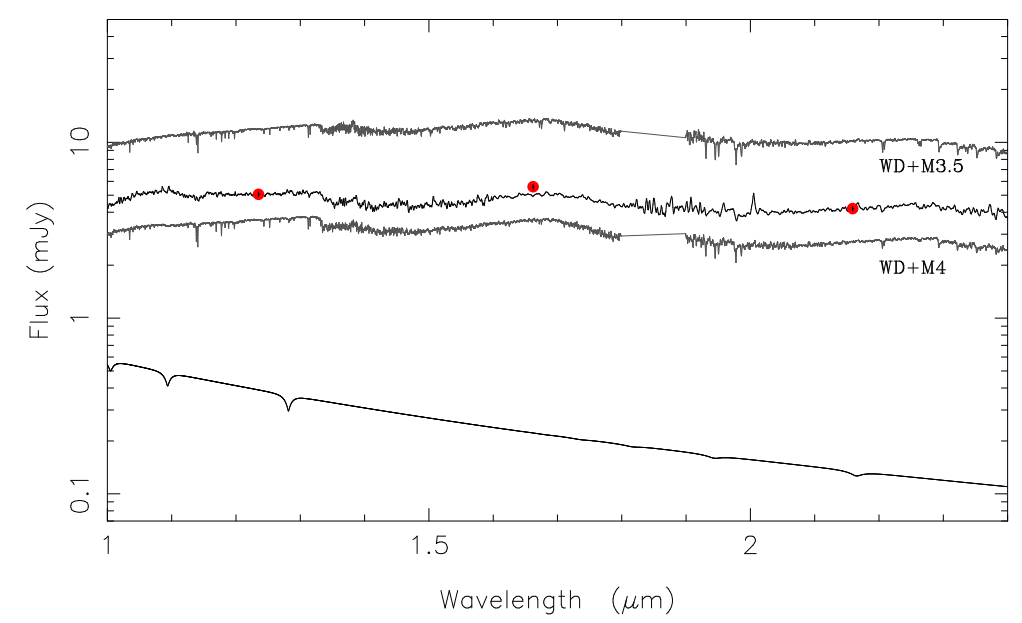

FIGURE 3.1. Model and observed spectrum of PHL 790 with composite models (dashed grey). 2MASS fluxes are also plotted (red). The dashed line indicates a region of very little atmospheric transmission.

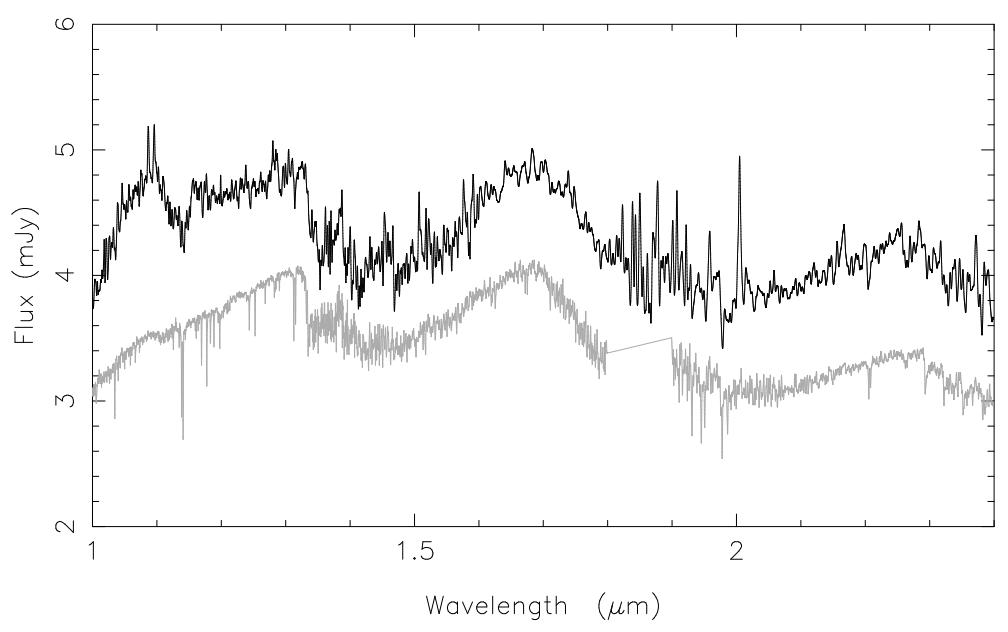

FIGURE 3.2. Model subtracted spectrum of PHL 790B with an M4 star (grey) for comparison. The dashed line indicates a region of very little atmospheric transmission.

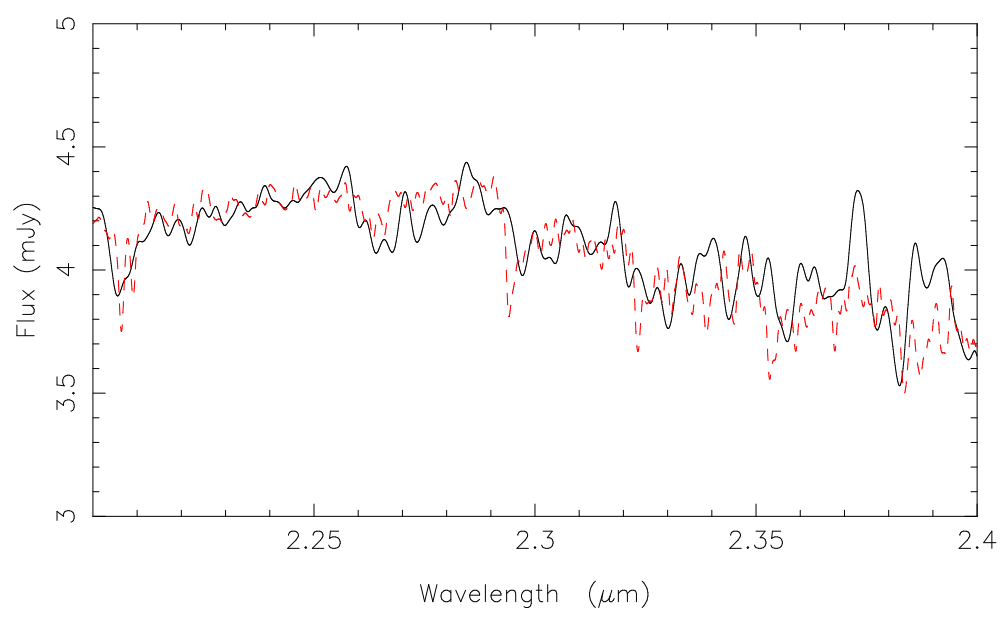

FIGURE 3.3. Model subtracted spectrum of PHL 790BB showing both the $\mathrm{Na}$ I and $\mathrm{CO}$ absorption lines. An M4 star is overplotted for comparison (red). 


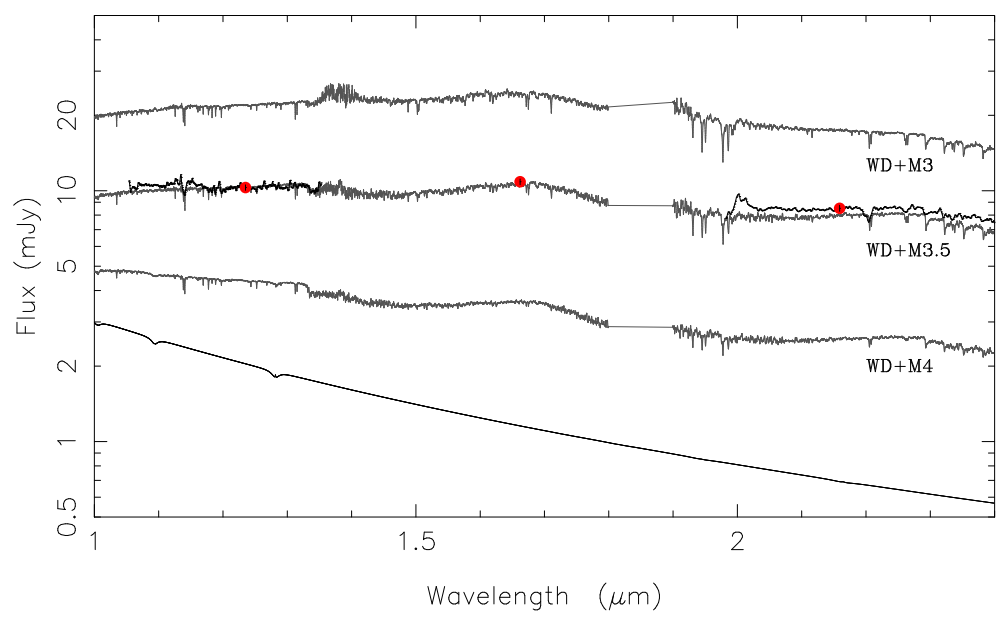

FIGURE 3.4. Model and observed spectrum of GD 984 with composite models (dashed grey). 2MASS fluxes are also plotted (red). The dashed line indicates a region of very little atmospheric transmission.

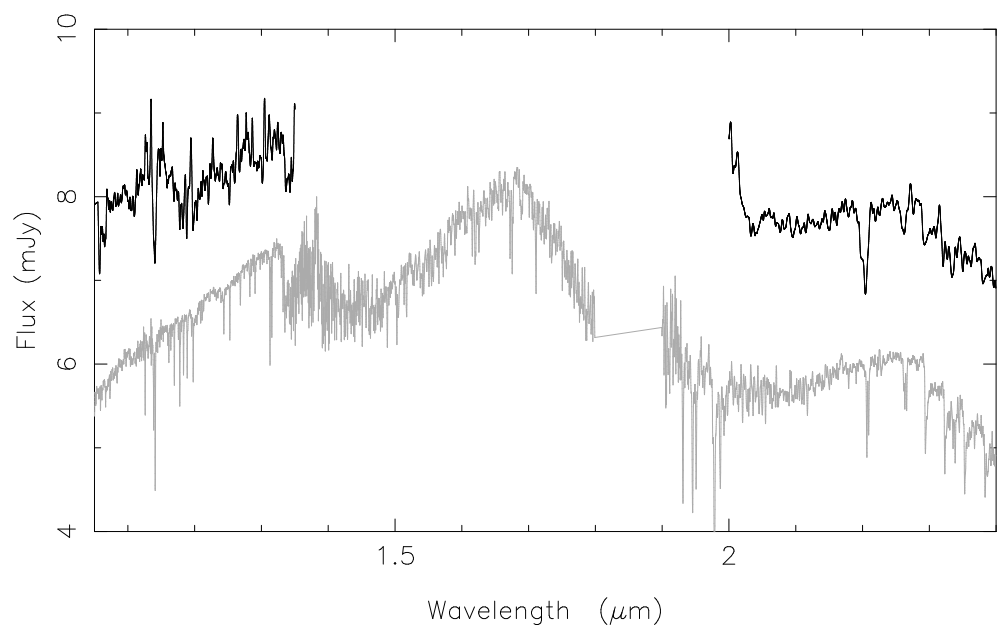

FIGURE 3.5. Model subtracted spectrum of GD 984B in $J$ and $K$ bands, with an M3.5 star (grey) for comparison. The dashed line indicates a region of very little atmospheric transmission.

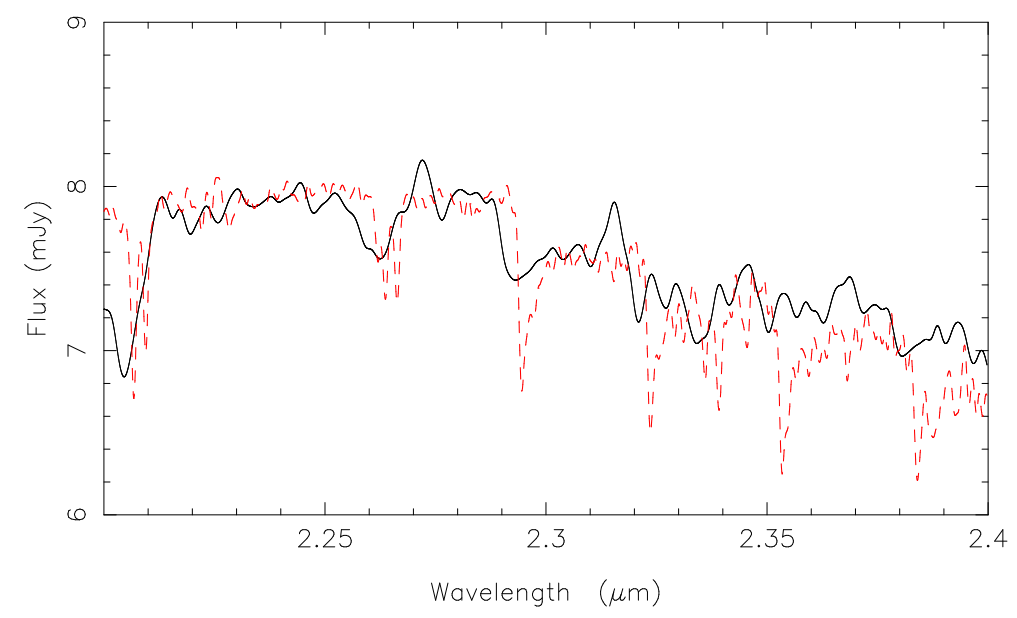

FIGURE 3.6. Model subtracted spectrum of GD 984B showing both the Na I and CO absorption lines. An M3.5 star is overplotted for comparison (red) 
Table 3.4. Summary of results to the near-infrared spectroscopic identification giving the spectral type of each companions. Also shown are previous photometric estimates and projected orbital distances.

\begin{tabular}{llccc}
\hline WD & Names & $\begin{array}{c}\text { Infrared } \\
\text { Spectral Type }\end{array}$ & $\begin{array}{c}\text { Photmetric } \\
\text { Spectral Type }\end{array}$ & $\begin{array}{c}\text { Projected Orbital } \\
\text { Orbital Seperation (AU) }\end{array}$ \\
\hline \hline $0017+061$ & PG 0017+061 & M4 & M4 & 266 \\
$0131-163$ & GD 984, REJ 0134-160 & M3.5 & M3.5 & $<60$ \\
$0419-487$ & V* RR Cae, LTT 1951 & M4 & M4 & - \\
$0752-146$ & LTT 2980 & M6-6.5 & M6 & $<0.1$ \\
$1305+018$ & PG 1305+018 & M4-5 & M1-M3.5 & $<96$ \\
$1412-049$ & PG 1412-049 & M1 & M0 & 1200 \\
$1415+132$ & PG 1415+132, Feige 93 & M3.5 & - & - \\
$1622+323$ & PG 1622+323 & M0.5 & M1 & 49 \\
$1643+143$ & PG 1643+143 & M2 & M2 & $<75$ \\
$1845+019$ & REJ 1847+015, Lanning 18 & M4 & - & - \\
$2151+015$ & LTT 8747 & M8 & M8 & 23 \\
\hline
\end{tabular}




\subsubsection{Constraining Spectral Types Through Na I Line Strengths}

These estimates of the spectral type can be tested by measuring the equivalent width of a suitable absorption line in the subtracted spectrum of the secondary. For the sample, the $\mathrm{NaI} 2.2 \mu \mathrm{m}$ line was chosen as it shows a distinctive temperature dependence whilst being independent of luminosity class (Section 3.4.2). It is also the only absorption line present in every single star in this sample. The temperatures of the secondaries can be estimated based on their spectral type assigned from this work. Therefore, if the estimated temperatures do not correlate with the equivalent width of $\mathrm{Na}$, then the spectral type may have been under or overestimated. However, this may also be evidence for irradiation of the companion by the primary, which would result in an earlier spectral class being assigned due to excess flux. Equivalent widths were measured using the spectrum analysis software DIPSO. The results are recorded in Table 3.5 and plotted in Figure 3.7 along with stars from Jones et al. (1994), Ivanov et al. (2004) and Tappert et al. (2007) for comparison.

The stars from the previous work show a roughly linear relationship with the equivalent width of the $\mathrm{Na}$ I line decreasing with increasing effective temperature. Although there is some scatter, the stars in this new sample would appear to follow the same trend to within $3 \sigma$ of the calculated errors in equivalent width. Thus, the spectral types assigned in the near-infrared and thus the estimated effective temperatures of the secondaries agree with the measured values of the $\mathrm{Na}$ I equivalent width. 
Table 3.5. Equivalent width of the NaI lines at $2.2 \mu \mathrm{m}$ for each companion

\begin{tabular}{lc}
\hline Name & EW NaI $(2.2 \mu \mathrm{m})(\AA)$ \\
\hline \hline WD 0017+061b & $3.9 \pm 0.9$ \\
WD 0131-163b & $7.5 \pm 0.6$ \\
WD 0419+105b & $6.5 \pm 0.5$ \\
WD 0752-146b & $8.9 \pm 0.5$ \\
WD 1305+018b & $4.7 \pm 0.5$ \\
WD 1412-049b & $5.6 \pm 1.0$ \\
WD 1415+132b & $3.0 \pm 0.4$ \\
WD 1622+323b & $7.9 \pm 0.6$ \\
WD 1643+143b & $3.5 \pm 0.5$ \\
WD 1845+019b & $7.0 \pm 0.6$ \\
WD 2151-015b & $9.6 \pm 0.4$ \\
\hline
\end{tabular}


Figure 3.8 is a near-infrared two colour diagram using the 2MASS photometry of the targets. This has been subdivided into the regions described by Wellhouse et al. (2005) (See Section 2.3.2). This allows for the additional investigation into whether the secondaries have been irradiated by the white dwarf primary, causing an overestimation of temperature in the targets (Tappert et al. 2007). Irradiation would make the secondary stars appear bluer and shift them to the lower left of Figure 3.35 in to region I. It can be seen that most of the secondaries lie within region II, exactly where white dwarfs with red companions are expected to appear (Section 2.3.2). The exception is WD $1845+019$ which lies in region III. However, this the faintest target and its position in the diagram is $<2 \sigma$ from region II. 


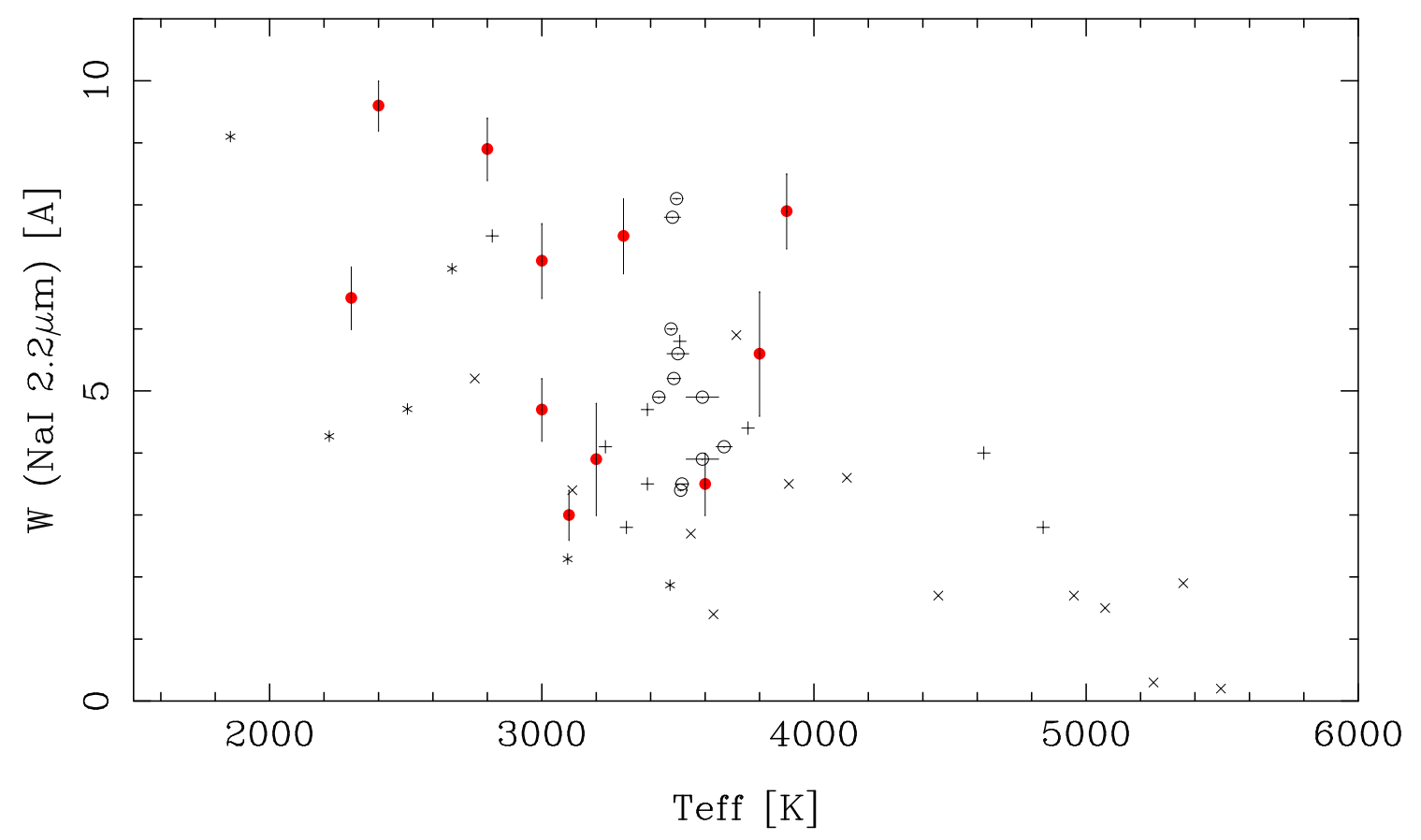

FIGURE 3.7. Equivalent width of $\mathrm{NaI}$ as a function of effective temperature. Filled circles are the secondaries from this work with temperatures estimates based on spectral type. Also plotted are stars from Jones (1994) $(\times)$, Ivanov et al. (2004) (+) and Tappert et al. (2007) (o). 


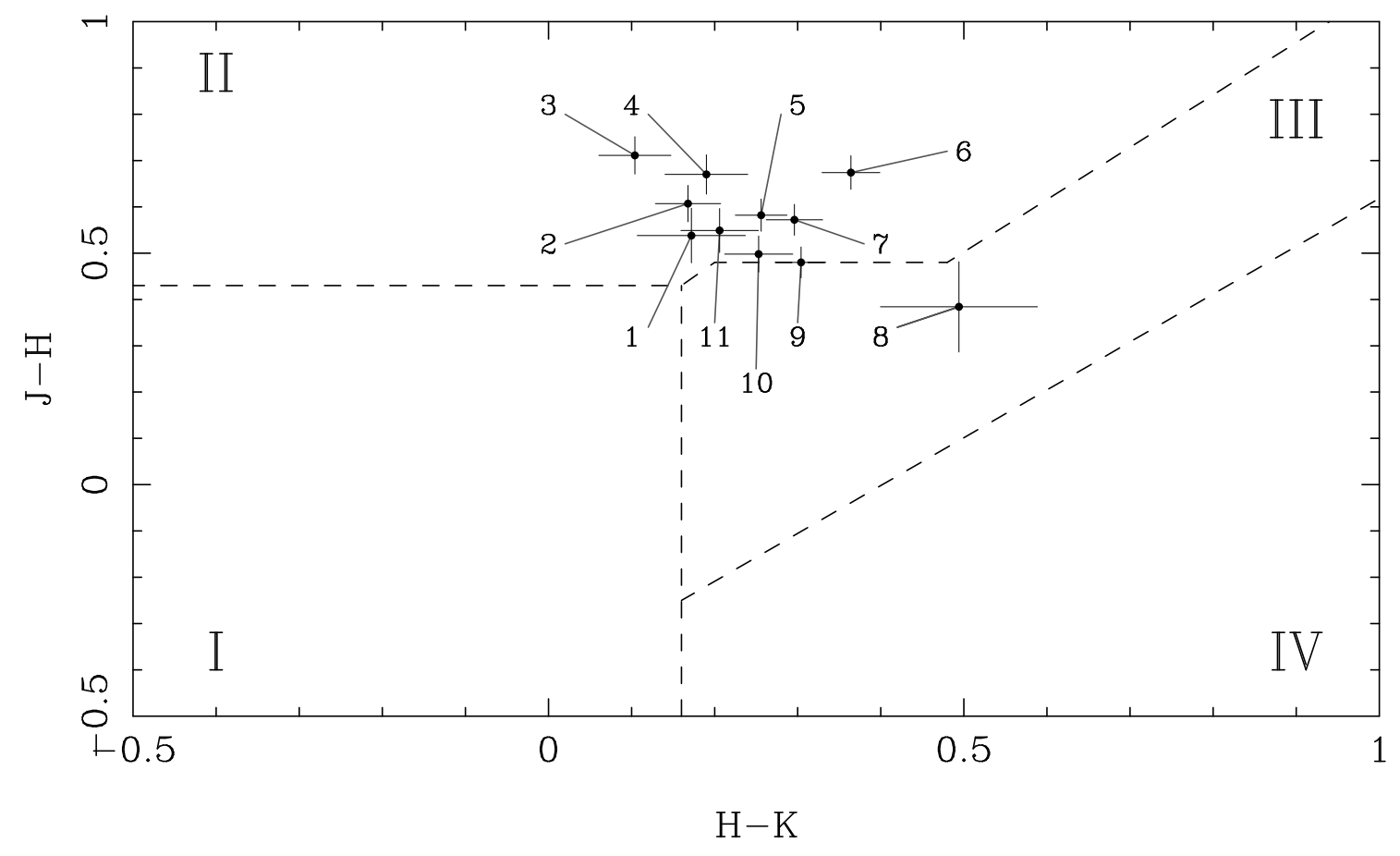

FIGURE 3.8. Near-IR two colour diagram for stars in this work divided in to the Wellhouse et al. (2005) regions delineating; (I) single white dwarfs, (II) white dwarfs with late-type main-sequence companions, (III) white dwarfs with lower mass, possible brown dwarf companions and (IV) white dwarfs contaminated with circumstellar material. Labelled as (1) WD $1415+132$ (2) WD $1643+143$ (3) WD $1412-049$ (4) WD $1622+323$ (5) WD $1305+018$ (6) WD $2151-015$ (7) WD $0419-487$ (8) WD $1845+019$ (9) WD $0752-$ 146 (10) WD 0131 - 163 (11) WD $0017+061$. 


\subsection{Summary}

I have determined the spectra type of the M dwarf companions of 11 DA white dwarfs using near-infrared spectra comparing key features in each star's spectrum with those observed in previously observed $\mathrm{M}$ dwarfs. The results are summarised in Table 3.4.

Six of the secondary star classifications agree with previous optical and near-infrared photometric typing. Where the spectroscopic results do disagree this has been no more than \pm 1 spectral types away from the previous estimate, which could be due to errors in distance estimates or activity intrinsic to the companion.

The accuracy of the spectral types have been tested by measuring the equivalent width of the $\mathrm{Na}$ I line at $2.2 \mu \mathrm{m}$, which shows a roughly linear relationship when compared to the effective temperature of the secondary. This shows that all of the secondary stars fall within errors of the expected trend between the equivalent width and effective temperature of the Na I line. This would suggest that intrinsic stellar activity or irradiation by the white dwarf primary is not affecting the estimate of the spectral type significantly, and that in many cases photometry is accurate to within \pm 1 when assigning a spectral type. All of the systems also have near-infrared colours expected of white dwarf + M-dwarf binary systems.

In systems where the companion is known to have a short period (i.e. a close orbit) or the period is currently unknown, the $\mathrm{CO}$ bands at $1.3 \mu \mathrm{m}$ have been visually inspected in comparison with a template star (as was the case in Tappert et al 2007) for evidence 
of accretion during a CE phase. As in Tappert et al. (2007) no evidence has been found in the sample for anomalous $\mathrm{CO}$ absorption indicating a previous $\mathrm{CE}$ phase or nuclear activity in the secondary. 


\section{Chapter 4}

\section{PG 1234+482: A Borderline Substellar Companion}

\subsection{Overview}

PG $1234+482$ (PG 1234) is a hot $\left(T_{\text {eff }} \approx 55,000 \mathrm{~K}\right)$ DA white dwarf with some evidence for metal pollutants in its photosphere. Jordan et al. (1996) reported the detection of Fe in an Extreme Ultraviolet Explorer spectrum of the star, and Wolff et al. (1998) give the overall metallicity as $20 \%$ that of the archetypal metal-rich hot DA G191-B2B using the same data (a pure-H atmosphere over-predicts the EUV continuum flux and metal opacities are required to satisfactorily model these data). But Barstow et al. (2003) did not detect any heavy elements in a noisy International Ultraviolet Explorer far-UV spectrum, and only give upper limits on the abundances of $\mathrm{C}, \mathrm{N}, \mathrm{O}, \mathrm{Si}, \mathrm{Fe}$ and Ni. A quick analysis of the FUSE spectrum of PG 1234 also failed to reveal any obvious photospheric metal lines. 
PG 1234 was first observed in the near-infrared by Green et al. (2000b), who reported a small $1.3 \sigma K$-band excess and thus did not claim any evidence for a companion. Debes et al. (2005) later noted significant $H$ and $K$-band excess from the more precise 2MASS photometry and suggested the presence of a companion spectral type M8V. More recently Mullally et al. (2006) measured a mid-infrared excess in two Spitzer IRAC bands $(4.5 \mu \mathrm{m}$ and $8.0 \mu \mathrm{m})$. They modelled the excess using a companion with $T_{\text {eff }}<2000 \mathrm{~K}$, assigning a spectral type of early L.

This chapter presents near-infrared spectroscopy for PG 1234 confirming the presence of the very low mass companion and constraining its spectral type and I discuss the possible substellar nature of the secondary.

\subsection{Observations}

PG 1234+482 was observed on March 5th 2007 using the Long-slit Intermediate Resolution Infrared Spectrograph (LIRIS) during service time on the $4.2 \mathrm{~m}$ William Hershel Telescope (WHT). LIRIS is a near-infrared imager/spectrograph which uses a $1024 \times$ 1024 pixel array HAWAII detector. The pixel scale is $0.25 \% /$ pixel, giving a field view of $4.27^{\prime} \times 4.27^{\prime}$. Data were acquired using the $H K$ grism providing a wavelength coverage of $1.39-2.42 \mu \mathrm{m}$. Observations were taken using the standard technique of nodding the point source targets along the spectrograph slit in an ABBA pattern. For PG 1234 $18 \times 100$ s exposures were taken (9 AB pairs) for a total exposure time of 1800 s, followed by $4 \times 10$ s exposures of an $\mathrm{A} 3 \mathrm{~V}$ telluric standard. The average airmass over the 
course of the observations was 1.06 .

\subsection{Data Reduction}

The data were first corrected for bottom-left quadrant pixel 'scrambling' (the image is constructed after readout with a 1-pixel dislocation) in all images using the lcpixmap function of the IRAF package LIRISDR ${ }^{1}$. Standard reduction techniques were then applied using software routines in the STARLINK packages KAPPA and FIGARO. In brief, a bad pixel map was constructed and applied to all the data. The science, standard star and arc lamp spectral images were flat fielded with a normalized response map. Difference pairs were then assembled from the science and standard star images and any significant remaining sky background was removed by subtracting linear functions, fitted in the spatial direction, from the data. The spectra of the white dwarf and the standard star were then extracted and assigned the wavelength solution derived from the arc spectrum. Any intrinsic features of the standard star's energy distribution were identified by reference to the near-infrared spectral atlas of fundamental MK standards (Wallace et al. 2000, Meyer et al. 1998, Wallace \& Hinkle 1997) and were removed by linearly interpolating over them. The spectrum of the white dwarf was then co-aligned with the spectrum of the standard star by cross-correlating the telluric features present in the data. The science spectrum was divided by the standard star spectrum and multiplied by a blackbody with $T_{\text {eff }}$ of the standard. Finally, the flux levels were scaled to achieve the best possible agreement between the spectral data and the $H$ and $K_{s}$ photometric fluxes

\footnotetext{
${ }^{1}$ http://www.iac.es/proyect/LIRIS/index.html
} 
of the object derived from the 2MASS All Sky Data Release Point Source Catalogue magnitudes (Skrutskie et al. 1995).

\subsection{Data Analysis}

To assist in spectrally typing the companion, the data were compared to composite white dwarf and $\mathrm{M} / \mathrm{L}$ dwarf models.

A pure $\mathrm{H}$ synthetic spectrum was calculated for $\mathrm{PG} 1234$ at $\mathrm{T}_{\text {eff }}=55,040 \mathrm{~K}$ and $\log g=7.78$ (Liebert et al. 2005) using the spectral synthesis codes TLUSTY and SYNSPEC (Section 2.3.3). The synthetic flux was normalized to $V=14.45$ (Liebert et al. 2005).

Empirical companion models were constructed as detailed in section 3.4.2. Subsequently, these model fluxes were re-scaled to be consistent with the Liebert et al. (2005) distance estimate of $144 \mathrm{pc}$. The final distance scaled models were then added to the synthetic white dwarf spectrum.

A comparison can also be made with the PG 1234 Spitzer photometry of Mullally et al. (2006) to archival Spitzer IRAC photometry of observed M and L dwarfs (Table 4.1; Patten et al. 2006). Again, these values have been appropriately scaled to the distance given previously. These fluxes have been added to expected values for PG 1234 calculated by integrating the synthetic spectrum folded though the appropriate filter transmission response. 
Table 4.1. Photometric fluxes in mJy of combined PG 1234 (Predicted) + M and L dwarfs compared to those of PG 1234 (Observed).

\begin{tabular}{lllllcc}
\hline & & & & \multicolumn{2}{c}{ Spitzer IRAC Channel } \\
Name & Spectral Type & $J$ (error) & $H($ error $)$ & $K_{\mathrm{s}}($ error $)$ & $4.5 \mu \mathrm{m}($ error $)$ & $8.0 \mu \mathrm{m}(\mathrm{error})$ \\
\hline PG 1234 (Observed) & DA & $1.628(28)$ & $1.067(21)$ & $0.707(13)$ & $0.2116(75)$ & $0.085(15)$ \\
PG 1234 (Predicted) + BRI 0021-0214 & DA + M9.5 & $1.549(27)$ & $1.035(20)$ & $0.729(13)$ & $0.240(10)$ & $0.010(18)$ \\
PG 1234 (Predicted) + 2MA 1439+1929 & DA + L1.0 & $1.512(26)$ & $0.946(19)$ & $0.631(12)$ & $0.191(8)$ & $0.072(12)$ \\
\hline
\end{tabular}




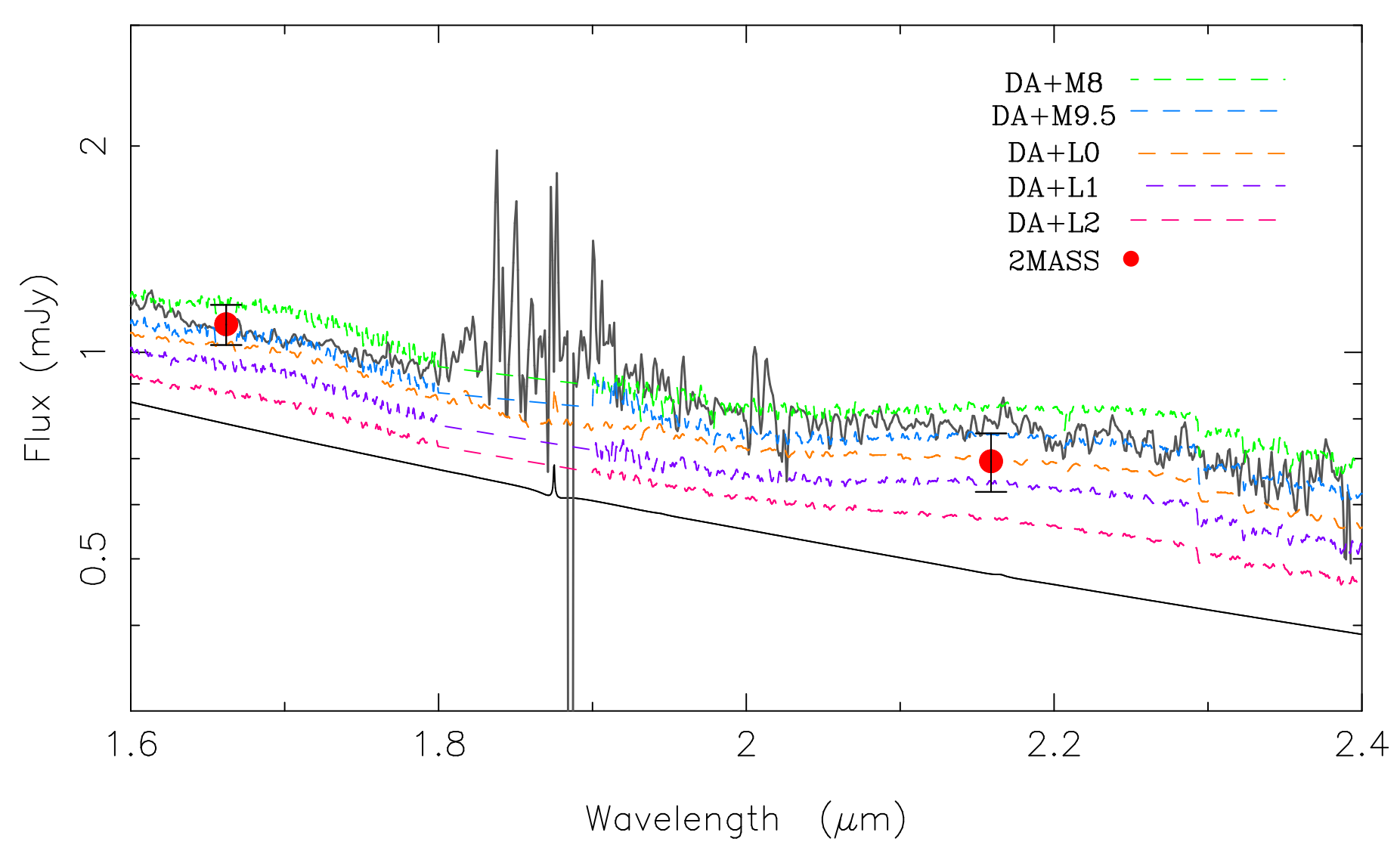

FIGURE 4.1. Observed near-infrared spectrum of PG 1234+482 scaled to the 2MASS $H$ flux. The predicted white dwarf model alone is shown by the solid line. The data are also compared to the white dwarf model combined with late $\mathrm{M}$ and early L dwarfs, all scaled to a distance of 144 pc (Liebert, Bergeron \& Holberg 2005). The upper and lower grey spectra are PG $1234+$ M8 and PG 1234 + L2 spectra respectively. The $1.87 \mu \mathrm{m} \mathrm{H}$ Paschen $\alpha$ emission line in the predicted white dwarf spectrum is due to non-LTE effects in the upper atmosphere. 


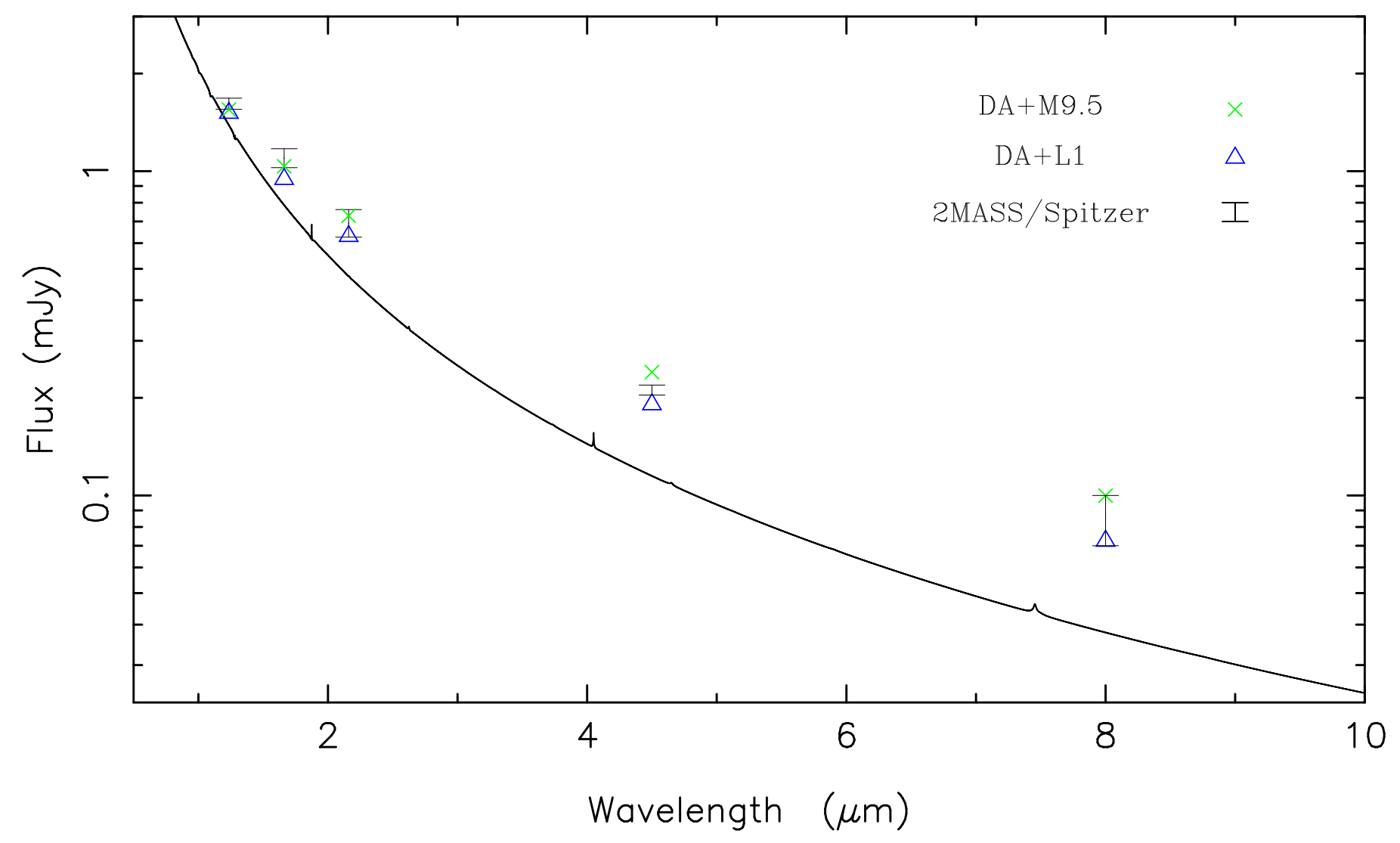

FIGURE 4.2. 2MASS and Spitzer IRAC $(4.5 \mu \mathrm{m}$ and $8.0 \mu \mathrm{m})$ photometric fluxes of PG 1234+482 with M9.5 and L1 fluxes scaled appropriately. The solid line is the white dwarf atmospheric model spectrum The symbols for the combined WD + M and L dwarf models do not represent the actual errors which can be seen in table 4.1. 


\subsection{Results}

Figure 4.1 shows the extracted spectrum for PG 1234. It is not possible to scale the spectrum to match both the $H$ and $K$ 2MASS photometry simultaneously, although when scaling to one the other is within $<2 \sigma$. This may indicate either a residual error in the reduction of the spectrum or errors in the 2MASS photometry. At $K_{s}=14.937 \pm 0.106$ PG 1234 does not meet the 2MASS Point Source Catalogue level 1 requirements (S/N $>10$ ) and Tremblay \& Bergeron (2007) have shown that lower quality 2MASS data should be treated with caution when interpreting near-infrared excesses to white dwarfs. In Figure 4.1 the spectral data are shown scaled to the $H$ photometry. Figure 4.1 also shows combined white dwarf + dwarf spectra of spectral types M9.5, L0 and L1. The light grey spectra above and below are an M8 and L2 respectively added to show the data do not match either of these types. When normalized to the $H$-band flux the observed spectrum is best approximated by a WD+M9.5 companion, whereas if normalized to the $K$-band flux the spectrum would be closer to that of a WD+L0 or WD+L1.

Figure 4.2 shows the 2MASS $J H K$ and the Spitzer IRAC $4.5 \mu \mathrm{m}$ and $8.0 \mu \mathrm{m}$ photometry (Mullally et al. 2006) of PG 1234 with the combined predicted PG1234 fluxes + BRI 0021-0214 (M9.5) and 2MA 1439+1929 (L1). It can be seen that the 2MASS fluxes are more closely approximated by the WD+M9.5 spectral type, whereas Spitzer fluxes are over-predicted by the WD+M9.5 model, but are under-predicted by the WD+L1 model. Thus the Spitzer data are likely best matched with a WD+L0. 


\subsection{A Boundary Stellar-Substellar Companion}

Whether the companion is substellar depends on its age, which can be estimated as follows. The mass of the progenitor to PG 1234 can be estimated using the initial-final mass relationship (Section 2.6). The mass of PG 1234 is $0.61 \pm 0.02 M_{\odot}$ (Liebert, Bergeron \& Holberg 2005) yielding an initial mass of $2.4 \pm 0.1 M_{\odot}$. An approximate main sequence lifetime can then be calculated using the equation of Wood (1992):

$$
\mathrm{t}_{\mathrm{ms}}=10\left[\left(\mathrm{M}_{*} / \mathrm{M}_{\odot}\right)^{-2.5}\right] \mathrm{Gyr}
$$

Therefore, the age of the system is $\approx 1$ Gyr as the cooling age is negligible.

The effective temperature of an object on the substellar boundary can be estimated using the DUSTY models of Chabrier et al. (2000) and Baraffe et al. (2002). At an age of $1 \mathrm{Gyr}$ and the commonly used upper mass limit for brown dwarfs of $0.075 M_{\odot}$ (Burrows et al. 2001), an effective temperature of $\approx 2200 \mathrm{~K}$ can be expected. At this temperature the observations of Vrba et al. (2004) suggest a spectral type of L1-L2, and Golimowski et al. (2004) an L1. Using the empirical formula of Stephens et al. (2001):

$$
\mathrm{T}_{\text {eff }}=2220-100 \mathrm{~L}_{\mathrm{n}} \text { where } \mathrm{L}_{\mathrm{n}}=\mathrm{L} 0-\mathrm{L} 8
$$

a spectral type of L0-L1 is expected. Thus, the expected spectral type of a 1 Gyr object 
on the substellar boundary is $\approx \mathrm{L} 1 \pm 1$.

Therefore, there is not sufficient evidence to state conclusively whether the companion is stellar or substellar in nature - i.e. a brown dwarf. To further constrain the spectral type a higher resolution, higher $\mathrm{S} / \mathrm{N} H$ and more importantly $K$-band spectroscopy is needed. An accurate spectral type could then be determined from the relative strength of the $\mathrm{CO}$ absorption edges at $2.3 \mu \mathrm{m}$.

\subsection{Summary}

The spectral type of the low mass companion to PG $1234+482$ has been estimated using $H$ and $K$-band spectroscopy obtained from the WHT LIRIS instrument and the Spitzer IRAC $4.5 \mu \mathrm{m}$ and $8.0 \mu \mathrm{m}$ photometry. Due to uncertainty in the 2MASS photometry used to place these data on an absolute flux scale, an estimated spectral type of $\mathrm{L} 0 \pm 1$ (M9L1) was assigned, making PG 1234 the hottest and youngest $\left(\mathrm{t}_{\mathrm{cool}} \approx 10^{6} \mathrm{yr}\right.$; Liebert, Bergeron \& Holberg 2005) DA white dwarf with a possible brown dwarf companion.

The confirmation of a very low mass, possibly substellar, companion to PG 1234 leads to the speculation that the metals in the hot white dwarf's atmosphere are at least in part being accreted from the companion's wind. However, stars of a comparable effective temperature can exhibit photospheric metals due to radiative levitation (Barstow et al. 2003). To resolve this issue a constraint is needed on the separation of the pair, by either high resolution imaging and/or radial velocity measurements. Farihi et al. (2005) 
did not resolve the two, indicating that the separation is less than or approximately 1". Consideration of the post-main sequence evolution of these systems and observations by Farihi, Hoard \& Wachter (2006b) suggests that there may be a bimodal distribution of orbital separations among binaries containing at least one white dwarf: wide pairs with orbits $>10 \mathrm{AU}$ and very close systems $(<$ few solar radii) in which the companion was dragged in during the common envelope phase. The failure to resolve the system by Farihi et al. (2005) might be indicating that PG 1234 is a close binary, but it should be cautioned that the pair also may be aligned by chance.

Whether it is wide or close, and a possible survivor of common envelope evolution, PG $1234+482$ B is one of the lowest mass companions to a white dwarf currently known. A list of the known lowest mass detached companions to white dwarfs is shown in Table 4.2, and the lowest mass companions to CV's in Table 4.3. 
Table 4.2. The lowest mass detached companions to white dwarfs.

\begin{tabular}{llll}
\hline Name & SpTypes & Separation & Ref. \\
\hline WD 2151-015 & DA+M8 & 23AU & 1 \\
WD 2351-335 & DA+M8 & 2054AU & 2 \\
WD 1241-010 & DA+M9 & 284AU & 2 \\
PG 1234+482 & DA+L0 \pm 1 & Unresolved & 3 \\
GD 165 & DA+L4 & 120AU & 4 \\
GD 1400 & DA+L6/7 & Unresolved & 5,6 \\
WD 0137-349 & DA+L8 $\left(0.053 M_{\odot}\right)$ & 0.65R $R_{\odot}(\mathrm{P}=116$ mins $)$ & 7,8 \\
PHL 5038 & DA+L8 & 55AU & 9 \\
\hline
\end{tabular}

1. Farihi, Hoard \& Wachter (2006b) 2. Farihi et al. (2005) 3. This work 4. Becklin \& Zuckerman (1988) 5. Farihi \& Christopher (2004) 6. Dobbie et al. (2005) 7. Maxted et al. (2006) 8. Burleigh et al. (2006a) 9. Steele et al. (2009)

Table 4.3. The lowest mass companions to CV's

\begin{tabular}{llll}
\hline Name & SpTypes & Period & Ref. \\
\hline OY Car & CV+M6 & 91 mins & 1 \\
EX Eri & CV + L0.084 $M_{\odot}$ Star & $\sim 90$ mins & 2 \\
SDSS 1212 & DA+L8-T2 & 88.4 mins & 3,4 \\
SDSS 1035 & CV $+0.052 M_{\odot}$ BD & 82 mins & 5 \\
SDSS 1507 & CV $+0.056 M_{\odot}$ BD & $66.61 \mathrm{mins}$ & 6 \\
\hline
\end{tabular}

1. Wood \& Horne (1990) 2. Feline et al. (2004) 3. Burleigh et al. (2006a) 4. Farihi et al. (2008) 5. Littlefair et al. (2006) 6. Littlefair et al. (2007) 


\section{Chapter 5}

\section{PHL 5038: A Benchmark White Dwarf + L-dwarf Binary}

\subsection{Overview}

PHL 5038 was first reported by Haro \& Luyten (1962) in a photographic search for faint blue stars near the South Galactic Pole with $B=18 \mathrm{mag}$, and a poorly constrained position of $22^{\mathrm{h}} 20.5^{\mathrm{m}}-00^{\circ} 43^{\prime}(\mathrm{J} 2000)$. This star was recovered in the Sloan Digital Sky Survey as SDSS J 222030.68-004107.3 (Eisenstein et al. 2006), a white dwarf with $T_{\text {eff }} \approx 8037 \mathrm{~K}$ and $\log g=8.28$, yielding an accurate position for the white dwarf.

The white dwarf was detected in the UKIDSS Large Area Survey (LAS), with DR5 photometry for PHL 5038 listed in Table 5.1. These values give an excess of $7-10 \sigma$ when compared to the predicted values for the WD of $(J, H, K)=(16.86,16.72,16.72) \mathrm{mag}$, calculated by folding the WD model through the appropriate UKIDSS filter response curves. 
Table 5.1. Near-Infrared photometry of the PHL 5038 binary system and nearby calibration star.

\begin{tabular}{cccccc}
\hline \hline Bandpass & $\begin{array}{c}\lambda \\
(\mu \mathrm{m})\end{array}$ & $\begin{array}{c}\text { PHL 5038 } \\
(\mathrm{mag})\end{array}$ & $\begin{array}{c}\text { PHL 5038A } \\
(\mathrm{mag})\end{array}$ & $\begin{array}{c}\text { PHL 5038B } \\
(\mathrm{mag})\end{array}$ & $\begin{array}{c}\text { 2MASS J22203205-0040210 } \\
(\mathrm{mag})\end{array}$ \\
\hline$Y^{1}$ & 1.03 & $16.89 \pm 0.01$ & - & - & - \\
$J^{1}$ & 1.25 & $16.73 \pm 0.02$ & - & - & - \\
$H^{1}$ & 1.63 & $16.44 \pm 0.03$ & - & - & - \\
$K^{1}$ & 2.20 & $16.31 \pm 0.04$ & - & - & - \\
$H^{2}$ & 1.65 & - & $16.82 \pm 0.07$ & $17.84 \pm 0.08$ & $14.447 \pm 0.053$ \\
$K^{2}$ & 2.19 & - & $16.72 \pm 0.07$ & $17.18 \pm 0.08$ & $14.161 \pm 0.051$ \\
\hline
\end{tabular}

${ }^{1}$ UKIDSS passbands as described in Hewett et al. (2006). ${ }^{2} H$ - and $K$-band photometry were obtained on a single night. The photometric errors were calculated using a 5\% error from the nearby 2MASS star used for flux calibration, a $2-3 \%$ photometric measurement error, and an assumed $5 \%$ internal error typical of near-infrared arrays. The final column displays the nearby 2MASS calibration star with its 2MASS catalogue photometry and errors. Colour transformations between the filters are of the order of $1 \%$ (Carpenter 2001) and so are neglected. 
The UKIDSS data flag (mergedClass) indicated the possibility that PHL 5038 was an extended source at one or more bandpasses. Follow-up observations were warranted to investigate the nature of the excess flux and its possible spatially extended nature. Thus, this object was included in a Gemini programme to clarify the nature of near-infrared excesses to white dwarfs in the UKIDSS LAS (Chapter 2).

In this chapter I report on follow up near-infrared spectroscopy of PHL 5038 revealing a spatially resolved substellar companion of late L-type; only the second such pair found (over 20 years later) after GD 165AB

\subsection{Observations}

Near-infrared grism spectroscopy of PHL 5038 was obtained at Gemini North using NIRI (Hodapp et al. 2003). During the first acquisition images, the science target was spatially resolved into two components separated by 0.94 " (Position angle $=293.2$ "), and the slit was placed over both point sources for all subsequent programme observations. On $24^{\text {th }}$ August 2008, a single $30 \mathrm{sec} H$-band acquisition image was obtained prior to placement of the science targets on the slit. Spectroscopy was performed with the $H$ grism and the 6 pixel slit (covering $1.43-1.96 \mu \mathrm{m}$ at $R \approx 500$ ) with eight, single coadd frames of 225 secs each at one of two offset positions along the slit, for a total exposure time of 30 mins. The A0V telluric standard HIP 102631 was observed with eight frames of $1 \mathrm{sec}$ and three coadds. Internal flat field and arc lamp calibration frames were taken immediately following the science target spectroscopy. 
Three attempts were made to obtain $K$-band spectroscopy of PHL 5038. On $12^{\text {th }}$ August 2008, the science target was imaged for 30 secs at two offset positions, then placed on the slit, but the spectroscopic sequence was abandoned before usable data were taken. However, the acquisition image from this date could still be used for photometry. On $21^{\text {st }}$ August 2008, the target was also imaged prior to being placed on the slit. Spectroscopy was performed with the $K$ grism and the 6 pixel slit (covering $1.90-2.49$ $\mu \mathrm{m}$ at $R \approx 500$ ), with six, single coadd frames of 225 secs each at one of two offset positions along the slit, for a total integration time of 22.5 mins. The A0V telluric standard HIP 102631 was observed using three coadds of eight $1 \mathrm{sec}$ frames. Internal flat field and arc lamp calibration frames were taken immediately following the science target spectroscopy. Unfortunately, the fainter of the two point sources was poorly aligned with the slit, resulting in a significant loss of spectroscopic signal for that component (see below). On $6^{\text {th }}$ November 2008, an identical $K$-band imaging sequence preceded a successful placement of both point sources on the slit. However, due to poor seeing the system was not spatially well resolved, and the S/N was too low to merit an extraction for PHL 5038B.

\subsection{Data Reduction}

All NIRI frames were processed using Gemini IRAF niri and gnirs packages, including the creation of appropriate flat-field images, bad pixel masks, and wavelength calibrated spectral data. 


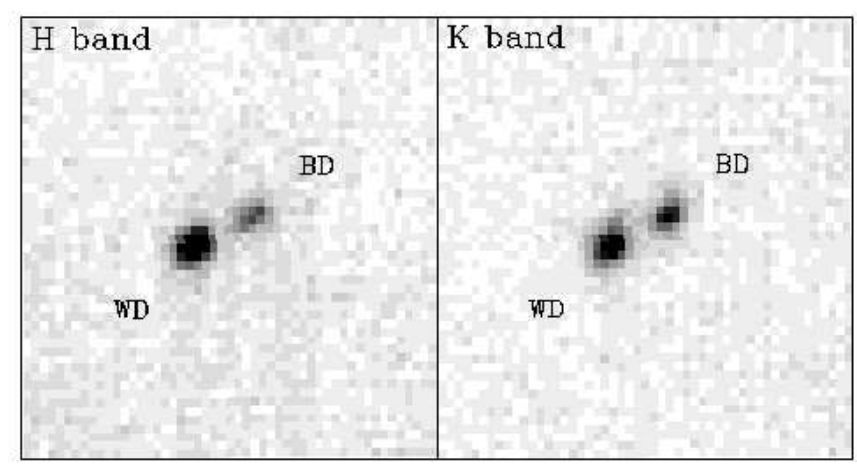

FIGURE 5.1. Near-infrared acquisition images of PHL 5038 taken with Gemini + NIRI immediately prior to $H$ - and $K$-grism spectroscopy. Frames are 6 " across with north up and east left. The brown dwarf companion, PHL 5038B, is located 0.94" from A at position angle 293.2", as measured in the $K$-band image shown above.

\subsubsection{Photometry}

Since only a single $H$-band image was obtained, this frame was cleaned of bad pixels and flat fielded. For the $K$-band image pairs, the frames were cleaned of bad pixels, flat fielded, pair subtracted, shifted and recombined. Fully reduced $H$ - and $K$-band images are shown in Figure 5.1; the WD is designated PHL 5038A and the cool companion is PHL 5038B.

Aperture photometry and astrometry on these images was performed with the IRAF task apphot using aperture radii of 2,3 , and 4 pixels $\left(0.117^{\prime \prime}\right.$ pixel $\left.^{-1}\right)$ and a sky annulus of 16-20 pixels. For most frames, photometry obtained with the 4-pixel aperture radius was discarded owing to probable contamination from the other binary component. A single $H$ - and $K$-band measurement was made for both PHL $5038 \mathrm{~A}$ and $\mathrm{B}$. The science target photometry was calibrated using a nearby (52" distant) field star, 2MASS J222032050040210, which fell onto the NIRI array in all imaging observations. Photometric data for PHL 5038 A and B, as well as the calibrator are given in Table 5.1. 


\subsubsection{Spectroscopy}

The spectroscopic frames were cleaned of bad pixels, flat fielded, pairwise subtracted, shifted, combined, and wavelength calibrated. A slightly more aggressive (relative to the default nscombine settings) outlier rejection algorithm was applied in the next to last step, which produced visibly superior spectral images for extraction. In the $H$-grism observations, the spectra of PHL 5038 A and B were well resolved spatially, and each was extracted using a 2-pixel spectral aperture. For the $K$-grism spectral extraction, only data from $21^{\text {st }}$ August 2008 were used. In these observations the two science target spectra were spatially resolved, but the signal of component B was significantly fainter than expected due to poor slit alignment. The data are still usable and a spectrum of each component was extracted using a 2-pixel spectral aperture, but care had to be taken with PHL 5038B, and the default aperture function had to be carefully corrected to avoid automatically picking up the signal of the WD. The telluric standard data was reduced and extracted identically.

Flux calibration and telluric feature correction were performed for each order with Spextool (version 3.3; Vacca et al. 2003, Cushing et al. 2004), using the general xtellcor package. Although in principle this should produce a well calibrated spectrum, there are a couple of reasons why this proved difficult or impossible in this case: 1) the telluric standard and science observations differed by 0.2 airmasses, and 2) slit losses; both ordinary and those caused by clear star-slit misalignment. Therefore the spectral data were calibrated from the Table 5.1 photometry; some inter-order adjustment was necessary for 
both the WD and the companion.

The spectral data were further processed to interpolate over data points where residual bad pixels or cosmic rays persisted (around several to one dozen), to crop the noise dominated edges, and to merge the data into a single, contiguous spectrum for each binary component. The fully processed spectra for PHL 5038A and B are shown in Figures 5.2 and 5.3 respectively.

\subsection{Data Analysis}

An model atmospheric fit of the SDSS spectrum of PHL 5038A using the models of Koester et al. (2005) yielded values of $T_{\text {eff }}=8000 \pm 100 \mathrm{~K}$ and $\log g=8.2 \pm 0.2$, implying a mass of $M_{\mathrm{WD}}=0.72 \pm 0.15 M_{\odot}$ and a distance of $64 \pm 10 \mathrm{pc}$. The model spectrum is plotted over the observed spectrum in Figure 5.2 and is consistent with the detection of $\mathrm{Pa}_{\alpha}$ absorption. No contamination from the secondary can be seen indicating a successful separation of the the components in the spectral analysis.

To determine the spectral type of PHL 5038B, the standard spectral indices for late L dwarfs and T dwarfs (Burgasser et al. 2006) were measured, and listed in Table 5.2 with established L8, L9 and T0 indices for comparison. The errors were determined by taking the standard deviation over the spectral index denominators for the $H$ - and $K$-band regions. These values indicate the spectral type is most likely L8-L9, with the $\mathrm{H}_{2} \mathrm{O}-\mathrm{H}$ index suggesting a spectral type of T0 is less likely . 


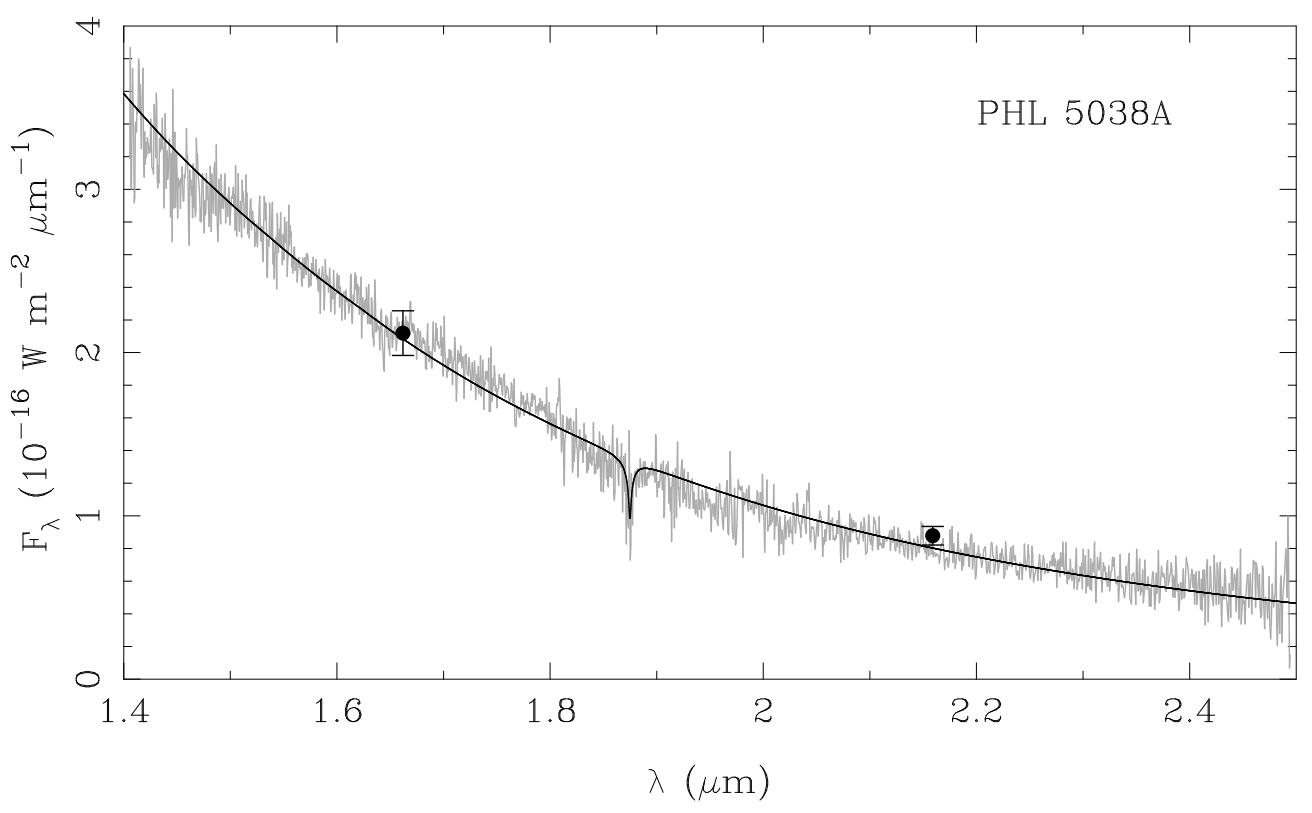

FiguRE 5.2. Modelled (black) and observed (grey) $H K$ spectra of PHL 5038 A plotted with photometry as measured in the acquisition images. Note the detection of $\mathrm{Pa}_{\alpha}$ absorption.

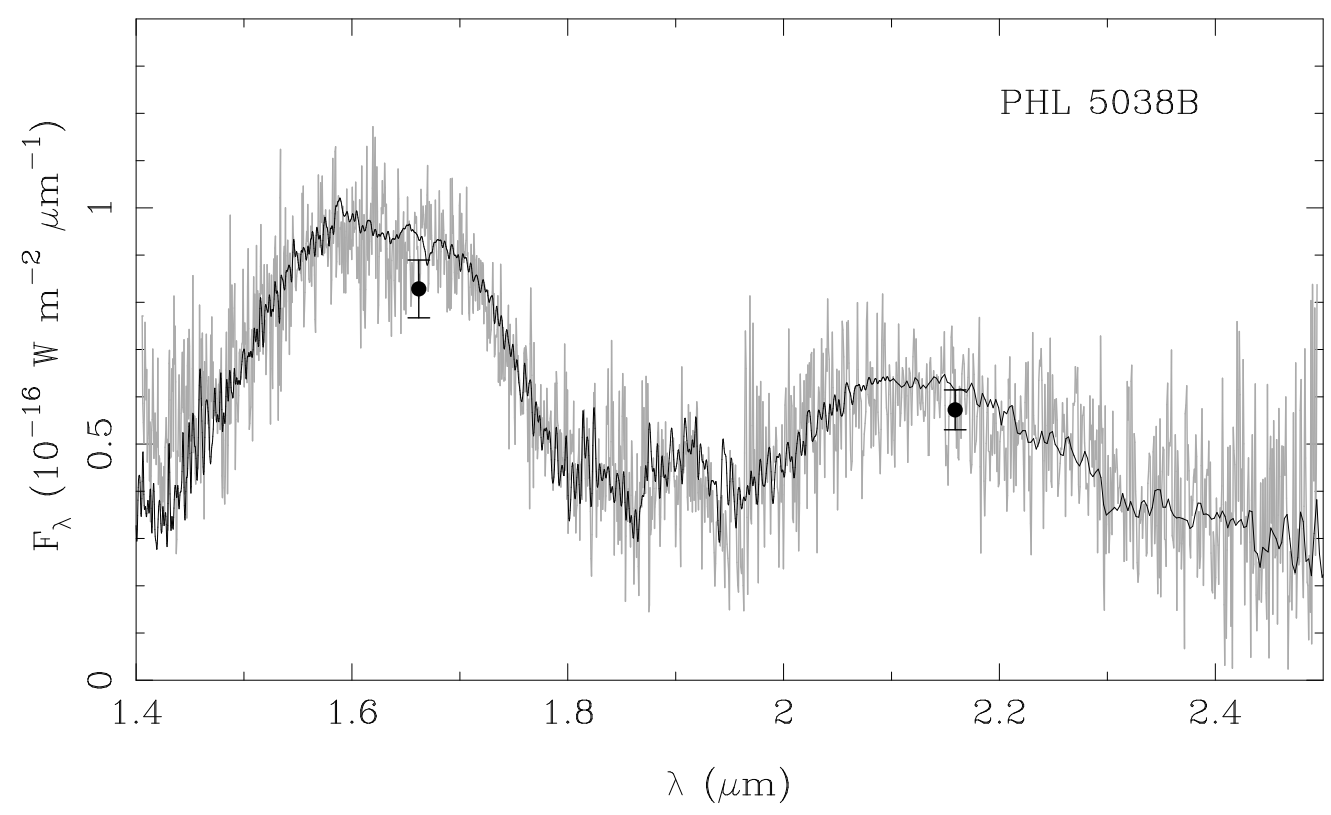

Figure 5.3. Observed $H K$ spectrum (grey) of PHL 5038 B plotted with photometry as measured in the acquisition images. An L8 spectrum (2MASS 1632+1904) has been scaled to match the flux of the secondary and over-plotted (black) for comparison. 
Table 5.2. Spectral indices as measured for PHL 5038 B, L8, L9 and T0 fields dwarfs (Burgasser et al. 2006).

\begin{tabular}{ccccc}
\hline \hline Index & PHL 5038 B & 2MASS 1632+1904 (L8) & DENIS 0255-4700 (L9) & SDSS 0423-0414 (T0) \\
\hline $\mathrm{H}_{2} 0-H$ & $0.74 \pm 0.08$ & 0.71 & 0.69 & 0.64 \\
$\mathrm{CH}_{4}-H$ & $0.98 \pm 0.08$ & 1.08 & 1.03 & 0.99 \\
$\mathrm{H}_{2} \mathrm{O}-\mathrm{K}$ & $0.76 \pm 0.14$ & 0.70 & 0.69 & 0.68 \\
$\mathrm{CH}_{4}-K$ & $0.89 \pm 0.14$ & 0.88 & 0.84 & 0.82 \\
\hline
\end{tabular}


The observed $H K$ magnitudes (Table 5.1) are also consistent with a late L-type BD at a distance of $64 \mathrm{pc}$. Although the $\mathrm{S} / \mathrm{N}$ of the spectrum is insufficient for the detection of any distinct absorption lines, the overall shape of the spectrum is also in best agreement with a spectral type of L8. The spectrum of an L8 field BD (2MASS1632+1904, McLean 2003) has been scaled to $64 \mathrm{pc}$ and plotted over the observed spectrum of PHL 5038B in Figure 5.3 for direct comparison.

\subsection{Discussion}

Caballero et al. (2008) list the spatial density of L8-L9 field dwarfs as $0.64 \times 10^{-3} \mathrm{pc}^{-3}$. It follows that the number of expected L8-L9 field dwarfs within a cylinder of angular radius $=1 "$ and length $=20 \mathrm{pc}$ centered on PHL 5038A is $\approx 3 \times 10^{-6}$ (Figure 5.4) Therefore, PHL 5038A and B are almost certainly a physical pair.

The 0.94 " angular separation of the binary, at the estimated distance of 64 pc equates to a projected orbital separation of $55 \mathrm{AU}$. When the main-sequence progenitor of PHL 5038A evolved into a WD the orbital separation of PHL 5038B expanded by a maximum factor of $\mathrm{M}_{\mathrm{MS}} / \mathrm{M}_{\mathrm{WD}}$ (Section 1.2). For $\mathrm{M}_{\mathrm{WD}}=0.72 M_{\odot}$ and $\mathrm{M}_{\mathrm{MS}}=3.2 M_{\odot}$, this factor would have been $\approx 4.4$. Thus, the original projected semi-major axis was $>13 \mathrm{AU}$.

The only other resolved L-type companion to a WD currently known is GD 165B, which has a projected orbital separation of $120 \mathrm{AU}$. Whether or not the secondary in this system is a true BD is still a matter of some debate (Zuckerman \& Becklin 1992, Kirk- 


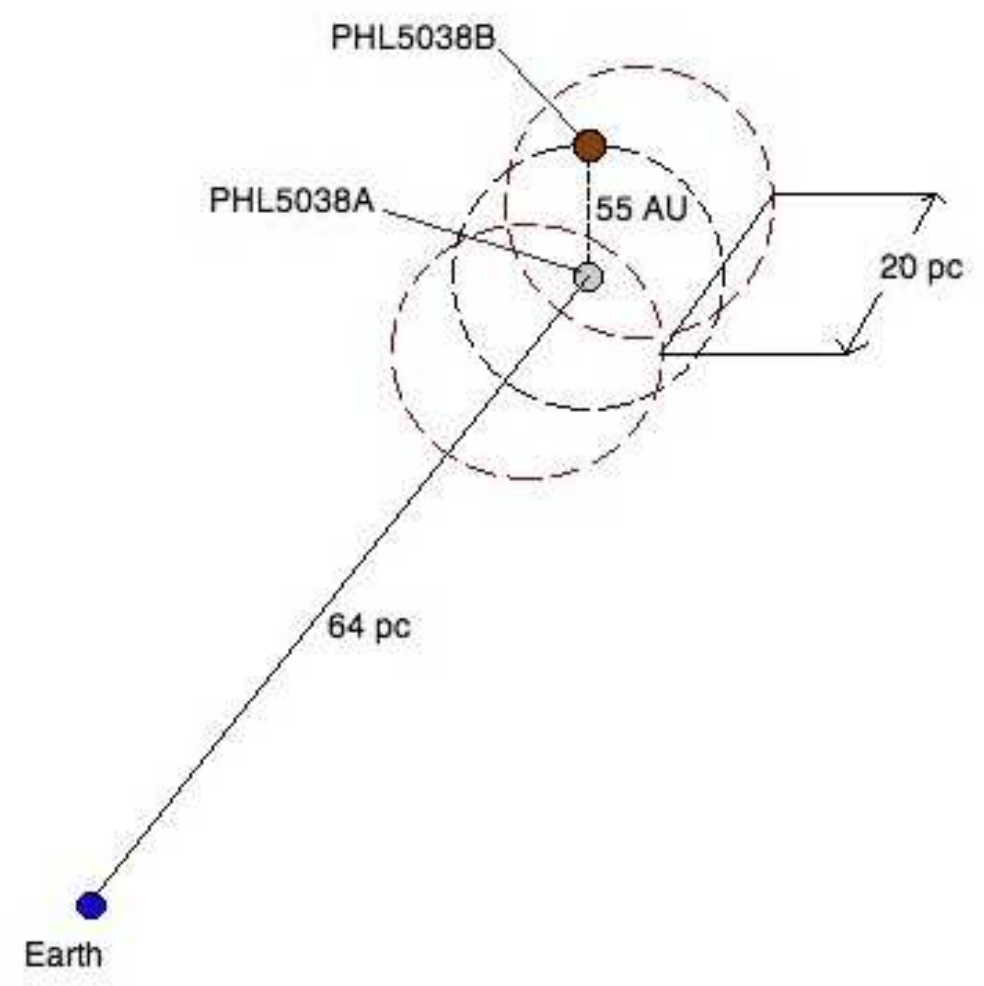

FIGURE 5.4. Spatial geometry for the PHL 5038 binary system. The expected number of field brown dwarfs of spectral type L8-L9 existing in the same region, defined by the distance errors, to PHL 5038A is $\approx 3 \times 10^{-6}$. 
Table 5.3. MS progenitor masses $\left(\mathrm{M}_{\mathrm{MS}}\right)$ and ages ( $\left.\mathrm{t}_{\mathrm{MS}}\right)$, WD cooling ages $\left(\mathrm{t}_{\mathrm{C}}\right)$, total age $\left(t_{\text {total }}\right)$, and MS age as a fraction of WD cooling age for PHL 5038A.

\begin{tabular}{cccc}
\hline & \multicolumn{3}{c}{$\mathrm{M}_{\mathrm{WD}}\left(M_{\odot}\right)$} \\
& 0.57 & 0.72 & 0.87 \\
\hline $\mathrm{M}_{\mathrm{MS}}\left(M_{\odot}\right)$ & 2.1 & 3.2 & 4.4 \\
$\mathrm{t}_{\mathrm{MS}}(\mathrm{Gyr})$ & 1.3 & 0.4 & 0.2 \\
$\mathrm{t}_{\mathrm{C}}(\mathrm{Gyr})$ & 1.0 & 1.5 & 2.5 \\
$\mathrm{t}_{\text {total }}(\mathrm{Gyr})$ & 2.3 & 1.9 & 2.7 \\
$\mathrm{t}_{\mathrm{MS}} / t_{\mathrm{C}}$ & 1.3 & 0.3 & 0.1 \\
\hline
\end{tabular}

patrick et al. 1993, Kirkpatrick et al. 1999, Zuckerman et al. 2003). The temperature of PHL 5038B is estimated to be $1230-1500 \mathrm{~K}$ by comparison to L-type field dwarfs with measured effective temperatures (Vrba et al. 2004). At an age of 1.9-2.7 Gyr (Table 5.3) the Lyon group models were interpolated to predict a mass of $\approx 49 \pm 3 \mathrm{M}_{\mathrm{J}}$ (Chabrier et al. 2000, Baraffe et al. 2002). Therefore, PHL 5038B is almost certainly a bona fide substellar object.

The mass luminosity relation for BDs is age dependent. Benchmark BDs can be used to test evolutionary models for substellar objects if their ages can be satisfactorily constrained (e.g., Dupuy et al. 2009). One of the candidates for these studies is widely orbiting WD+BD binaries (Farihi et al. 2005, Pinfield et al. 2006) such as PHL 5038. The limiting factor in this system is its age calibration. Pinfield et al. (2006) require that a good age calibrator's progenitor lifetime be no more than $10 \%$ of the WD cooling age $\left(\mathrm{t}_{\mathrm{MS}} / t_{\mathrm{C}}\right)$, due to intrinsic uncertainty in calculating main sequence (MS) lifetimes.

With $T_{\text {eff }}=8000 \pm 100 \mathrm{~K}$ and $\log g=8.2 \pm 0.1$ the cooling age of PHL 5038A is $1.0-2.5$ Gyr (Fontaine et al. 2001). The mass of the progenitor to PHL 5038A is estimated to be $3.2 \pm 1.1 M_{\odot}$ using the initial to final mass relationship of Dobbie et al. 
(2006) (Section 2.6). This indicates the WD descended from an A or late B-type star. An approximate main sequence lifetime can be calculated using Equation 4.1, giving $0.2-1.3$ Gyr. Therefore, the total age of the system is $1.9-2.7$ Gyr. These ages were calculated for the lower and upper mass limits of PHL 5038A and are listed in Table 5.3 along with the ratio $\mathrm{t}_{\mathrm{MS}} / t_{\mathrm{C}}$. This ratio ranges from $0.1-1.3$ for a $M_{\mathrm{WD}}=0.57-0.87 M_{\odot}$ and so only fulfils Pinfield's requirement for a benchmark BD if the WD mass is as high as $0.87 M_{\odot}$. At this point, it should be noted that the masses for DA white dwarfs cooler than $12,000 \mathrm{~K}$ derived from spectroscopic fitting techniques may be over-estimated by up to $0.2 M_{\odot}$, for reasons that are not fully understood. Koester (1991) discuss this effect, and suggest that it is due to problems with the atmospheric models, and in particular with the treatment of neutral particles. However, Bergeron et al. (1995) say that neutral particles only become a problem when the $T_{\text {eff }}<8000 \mathrm{~K}$, which is somewhat lower than the observed range. Koester et al. (2009) summarise these findings and conclude that more accurate modelling may indeed show an effect for DAs nearer $12,000 \mathrm{~K}$. As a result of this the mass of PHL 5038A is more likely to be in the range $0.65-0.75 M_{\odot}$.

\subsection{Summary}

I have confirmed the presence of and spectroscopically typed a BD companion to the WD PHL 5038A. PHL 5038B was assigned a spectral type of L8-L9 based on the measurement of standard spectral indices in the near-infrared, the first time this has been accomplished for a bona fide WD+BD binary. This spectral type is corroborated by the photometry of PHL 5038B and by comparison with the spectrum of an L8 field BD. The 
system is situated $64 \mathrm{pc}$ from Earth, and the BD has a projected orbital separation from its primary of $55 \mathrm{AU}$. PHL 5038B has the potential of being used as a benchmark BD if its ages can be determined more accurately. A measurement of a trigonometric parallax for this system would allow for a more accurate constraint on the radius and, via models, the mass of the WD. This would then allow a better estimate for the total age of the system. 


\section{Chapter 6}

\section{Discussion}

\subsection{Overview}

In this chapter I determine the latest spectral type of the lowest mass unresolved stellar or substellar (sometimes collectively known as ultracool) companion that could have been detected around each DA white dwarf in UKIDSS DR5. This data, along with the estimated distance to each star, is used to assess the overall sensitivity of UKIDSS to the detection of unresolved ultracool companions to DA white dwarfs. These results are then used in conjunction with the detected number of candidate L and T-dwarfs from Chapter 2 to estimate the fraction of L-dwarfs, T-dwarfs and brown dwarfs in binaries with DA white dwarfs. I compare these results with the previous estimate of Farihi et al. (2005) of the L-dwarf companion fraction, as well as previous estimates of the mainsequence + brown dwarf binary fraction. 


\subsection{Limits on Unresolved Ultracool Companions to DA White Dwarfs}

Atmospheric modelling successfully fitted 407 DA white dwarfs to within $3 \sigma$ of both their UKIDSS $H$ and $K$-band photometry. These stars will be used to estimate the limits on the spectral types of unresolved companions to DA white dwarfs and hence determine the sensitivity of UKIDSS to both L and T-dwarfs in white dwarf binaries. Only 10 DB white dwarfs were detected, which is not a sufficient number to accurately constrain binary statistics for helium atmosphere white dwarfs. The method used to calculate these limits is detailed below.

In order to place a limit on the spectral type of the coolest detectable unresolved companion to each DA white dwarf the absolute magnitudes for the spectral types of low mass objects ranging from L0-T8 are required. Patten et al. (2006) lists known M, L and T-dwarfs with 2MASS $J H K_{\mathrm{S}}$ photometry ranging from spectral types M5-T8 and also has measured parallaxes for many of these. The 2MASS $J H K_{\mathrm{S}}$ photometry for each object were converted to the UKIDSS $J H K$ photometric system using the colour corrections of Carpenter (2001). Taking only those with a measured parallax, and thus an estimated distance, the $K$-band photometry was scaled to $10 \mathrm{pc}$ for each spectral type to obtain an estimated absolute $K$-band magnitude. For stars of the same spectral type, an average of the magnitudes was used. For the few spectral types that had no parallax measurement a linear interpolation was performed to predict an absolute magnitude. The $K$-band magnitude was used for the reasons discussed in Section 2.3.4. 
For each apparently single DA white dwarf the expected $K$-band magnitude was predicted by folding the white dwarf's atmospheric model, or blackbody, through the UKIDSS $K$-band filter transmission profile. The observed $K$-band magnitude was not utilised, as any of the white dwarfs could be in excess within the observed errors. This would have resulted in an over-estimate of the limiting spectral type for the companion to these stars. A $3 \sigma$ detection limit was then calculated for each white dwarf by using the observed $1 \sigma$ errors from the UKIDSS DR5 database, and adding these to the predicted magnitudes. The distance to each white dwarf was calculated using Bergeron models (Section 2.3.3) using the effective temperatures and surface gravities produced from the automated fit of EIS06. For the 33 single DA white dwarfs only found in the McCook \& Sion catalogue, temperature estimates were obtained from the literature or an automated fit to the stars optical photometry. For stars without a measured surface gravity, a $\log g=8.0$ was assumed. This applied to only 11 white dwarfs in the sample, which is not a large enough number to create a distance bias that could later affect the statistics. Subsequently, the grid of M, L and T-dwarf absolute magnitudes were scaled to the estimated distance of each white dwarf and added to the predicted white dwarf $K$-band magnitudes until a match was made with the $3 \sigma$ detection limit. The spectral types of all the companion limits were summed (Table 6.1) and plotted as a histogram in Figure 6.1. A cumulative histogram is shown in Figure 6.2.

It should be noted that for the isolated white dwarf sample, the distances might be underestimated if they are, for example, unresolved double degenerate binaries. Thus, for this fraction $(\approx 5 \%$ unresolved WD+WDs, Holberg 2009$)$, the sensitivity to ultracool com- 
Table 6.1. Number of each spectral type placed as a lower limit to the 407 apparently single DA white dwarfs in UKIDSS DR5.

\begin{tabular}{cc|cc|cc}
\hline Spectral Type & No. & Spectral Type & No. & Spectral Type & No. \\
\hline \hline- & - & L0 & 0 & T0 & 38 \\
- & - & L1 & 6 & T1 & 36 \\
- & - & L2 & 4 & T2 & 42 \\
- & - & L3 & 5 & T3 & 20 \\
$<$ M5 & 0 & L4 & 14 & T4 & 25 \\
M5 & 1 & L5 & 20 & T5 & 21 \\
M6 & 1 & L6 & 19 & T6 & 14 \\
M7 & 2 & L7 & 31 & T7 & 14 \\
M8 & 2 & L8 & 35 & T8 & 8 \\
M9 & 1 & L9 & 40 & $>$ T8 & 8 \\
\hline
\end{tabular}




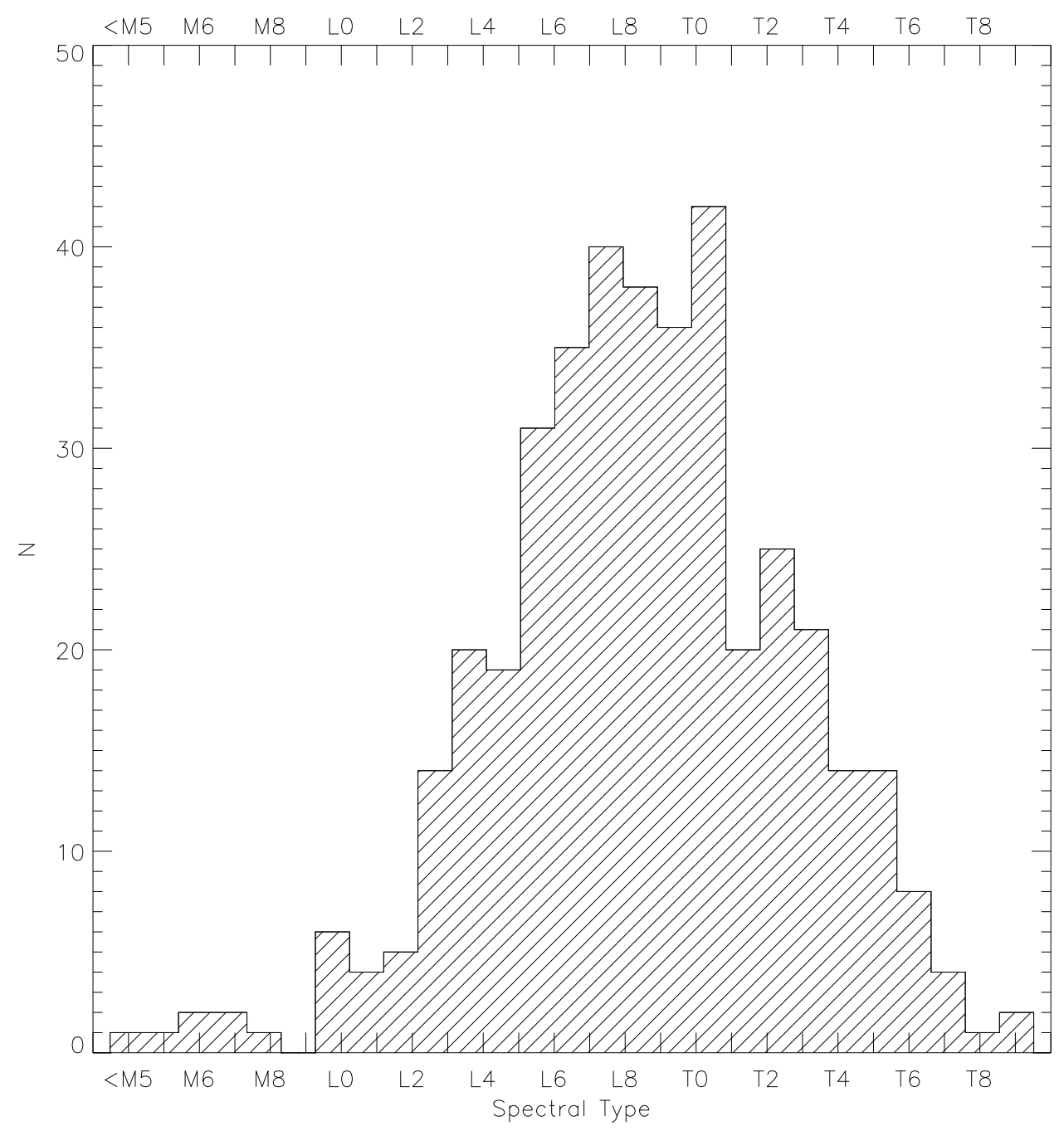

FIGURE 6.1. Distribution of limits on unresolved companions to the 407 "single" DA white dwarfs (with well determined parameters) detected in UKIDSS DR5. 


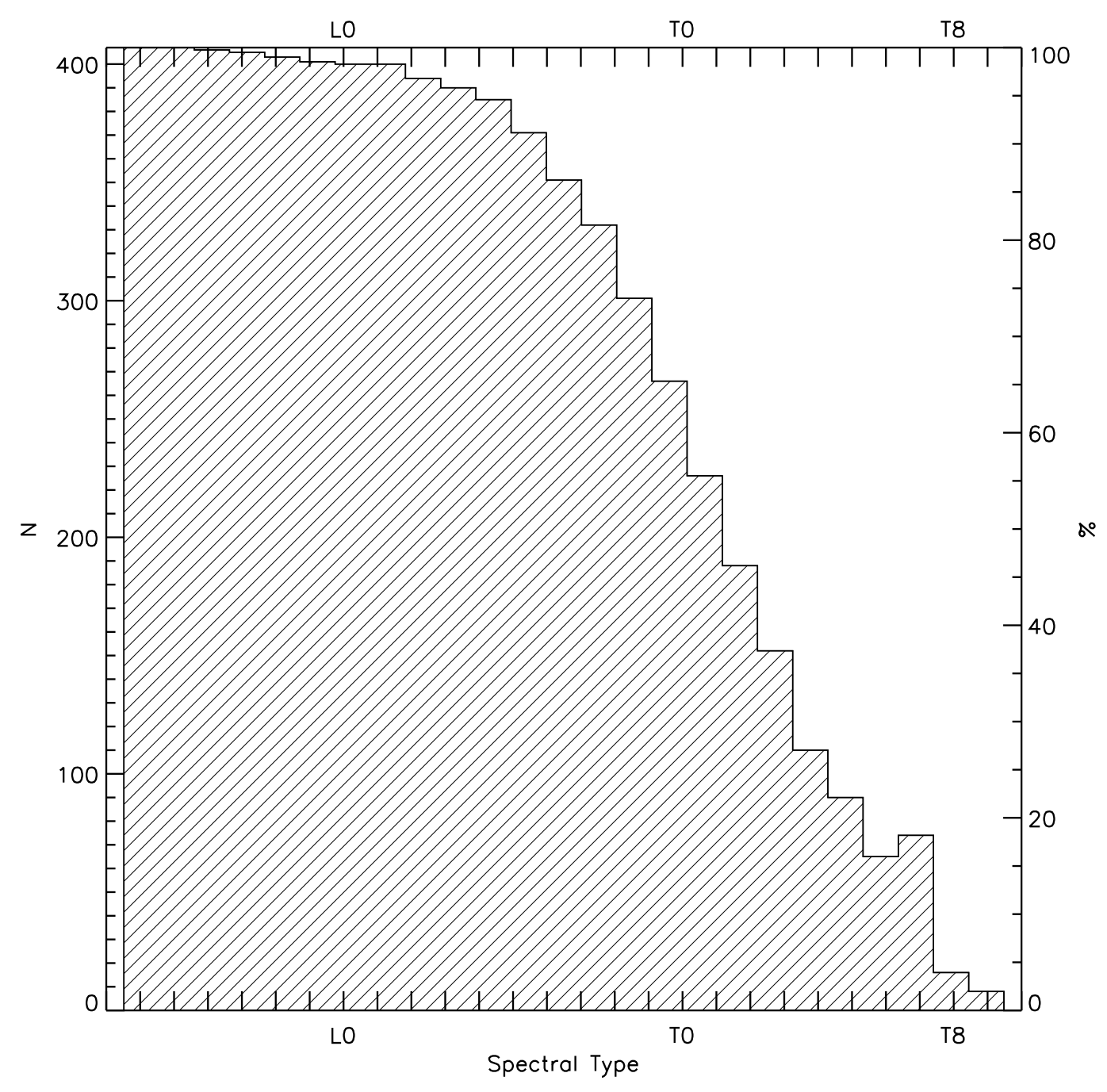

FIGURE 6.2. Same as Figure 6.1 but plotted as a cumulative distribution. 
panions may have been overestimated. It is also important to spectroscopically confirm the candidate secondary in each candidate binary system for the same reason i.e. in case the distance to the white dwarf has been under-estimated.

\subsection{Survey Sensitivity}

Figure 6.1 shows that the sensitivity of this survey for the isolated DA white dwarf sample peaks roughly at the L-T borderline, at approximately L8-T2. Figure 6.2 demonstrates that the sensitivity function then falls off as the limits approach a spectral type of late T, with only $56 \%$ of the sample sensitive to the detection of a companion earlier than or equal to spectral type T0. In contrast $98 \%$ of the sample is sensitive to the detection of a companion of spectral type earlier than or equal to L0, dropping to $65 \%$ by L9. Figure 6.3, shows a histogram of the distances to these single white dwarfs. $98 \%$ of the sample lies within $500 \mathrm{pc}$ of Earth.

In considering the total survey sensitivity, the $204 \mathrm{DA}+\mathrm{dM}$ binaries must be taken into account. These must be included in the final sample for statistical analysis as many of these systems would have been sensitive to the detection of L and T-type companions, if the M-dwarf companion were to be removed. However, the presence of the M-dwarf companions may bias this population towards greater distances, as the secondary is far brighter in the near-infrared. Therefore, the fraction of DA $+\mathrm{dM}$ binaries that lie within 500 pc must be determined so a direct comparison can be made against the isolated white dwarf population. The distances to these were estimated using the effective temperatures 


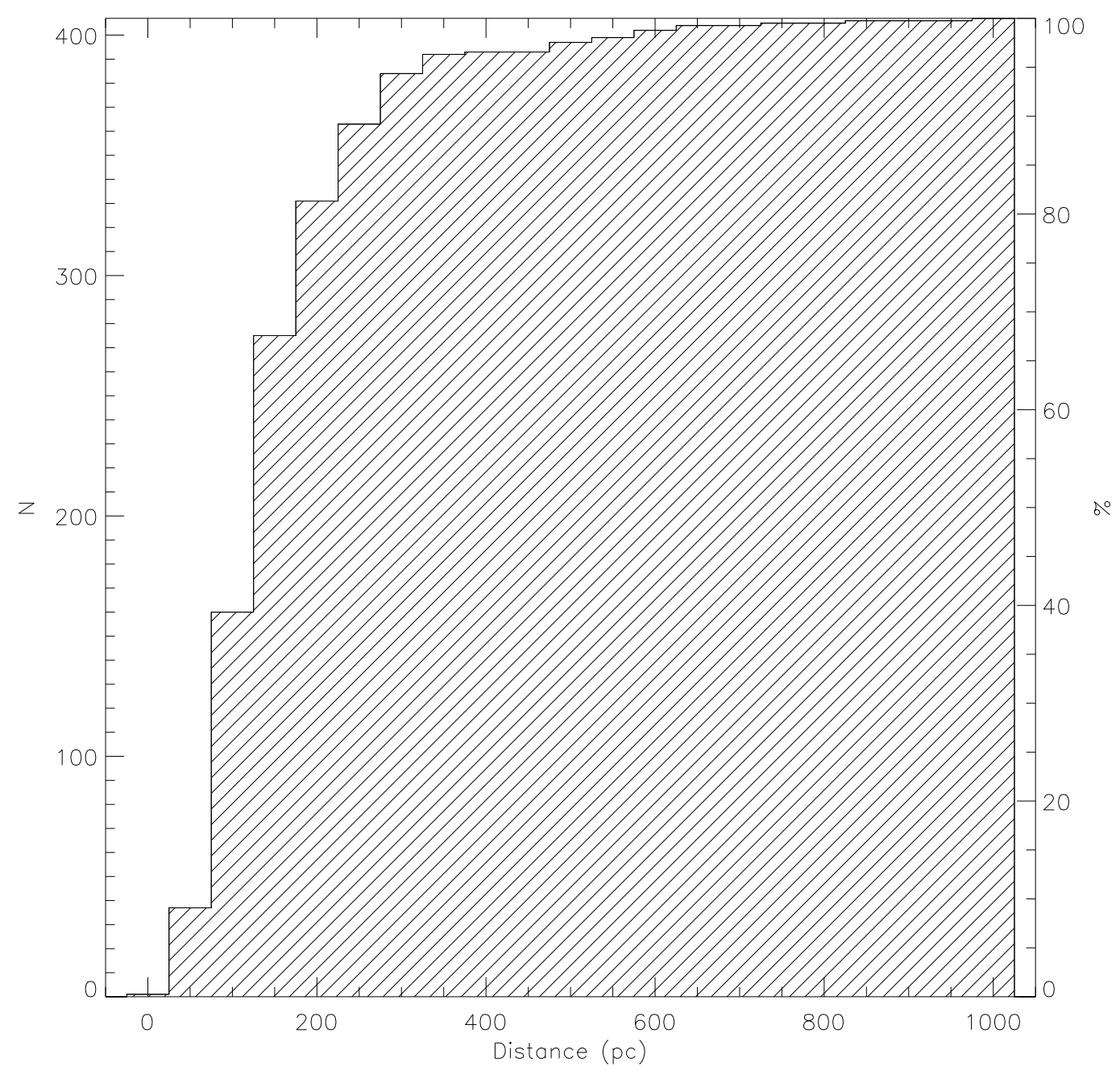

FIGURE 6.3. Cumulative distribution of distances to the 407 isolated DA white dwarfs in this work. 


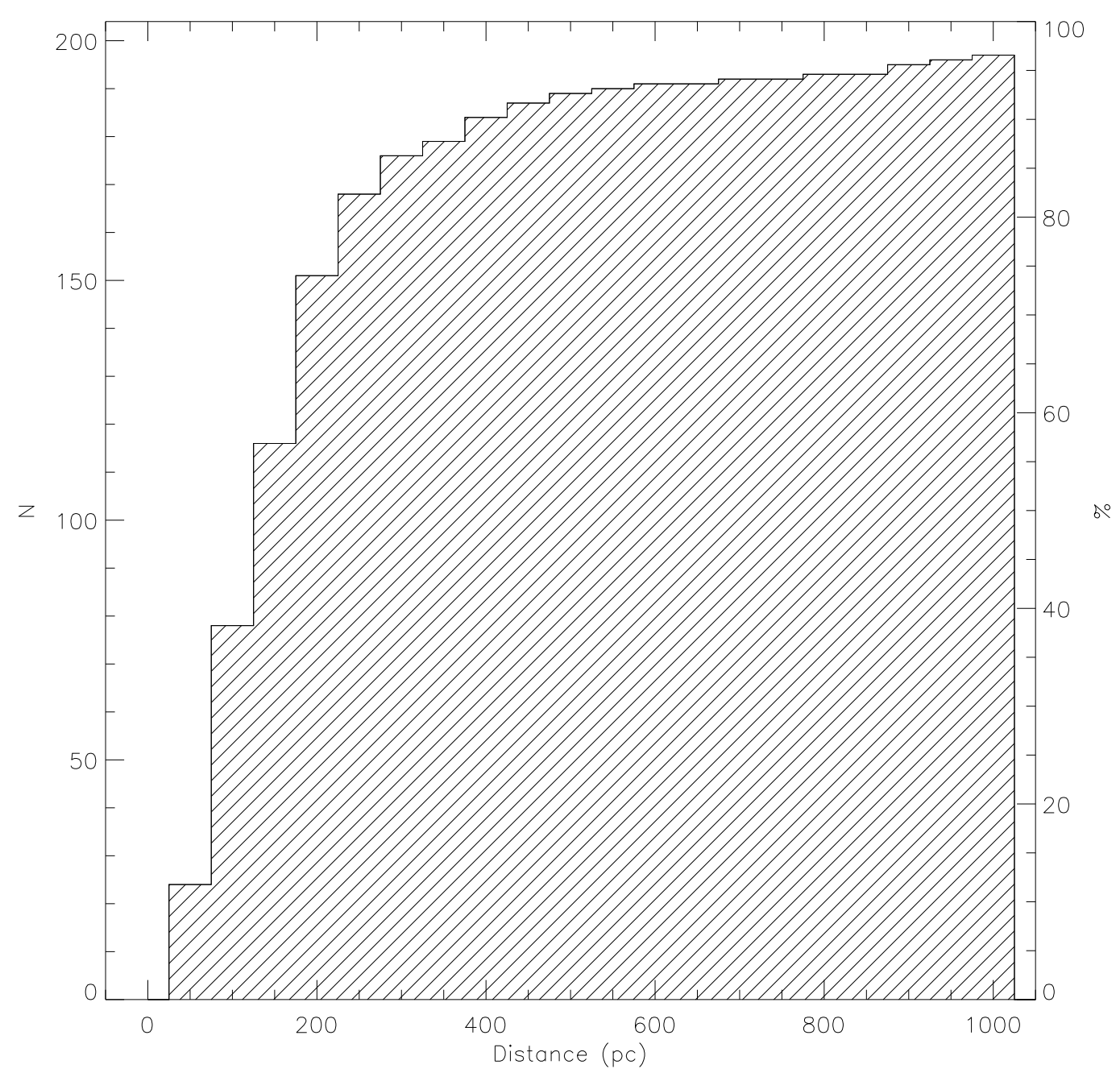

FIGURE 6.4. Cumulative distribution of distances to the 204 DA+dM binaries in this work. 
and surface gravities assigned by Eisenstein et al. (2006). This data is plotted as a histogram in Figure 6.4, with $93 \%$ of the DA+Ms lying within 500 pc. Therefore, there is not a significant difference between the isolated and white dwarf + M-dwarf distance distributions and the distance distribution of the isolated white dwarf sample will be assumed for the purpose of statistical analysis.

\subsection{Binary Statistics for White Dwarfs with Unresolved Substellar Companions}

In this section the fractions of DA white dwarfs with unresolved L-dwarf, T-dwarf and brown dwarf companions are going to be estimated.

It can be assumed that the white dwarf $+\mathrm{L}$ and T-dwarf candidates, and the candidate disk systems, follow the same distance distribution as the single white dwarfs, as distance estimates are based on parameters derived from the optical spectra, which are not significantly effected by the presence of an L or T-dwarf. The new candidate white dwarf + M-dwarf systems can be added to the previously identified WD+dM binary sample. Therefore, the complete sample size of DA white dwarfs at any distance, with or without a near-infrared excess is 641 . The number of these stars that lie within $500 \mathrm{pc}$ is $98 \%$ of the total sample size giving 628 DA white dwarfs.

An upper limit for the unresolved L-dwarf, T-dwarf and brown dwarf companion fractions to DA white dwarfs can now be calculated. This will be done firstly for the DA + 
L-dwarfs, followed by the DA + T-dwarfs and then finally for the DA + brown dwarf population.

The candidate $\mathrm{L}$ and T-dwarfs have been identified through near-infrared photometry with only PHL 5038 and SDSS J1212+013 confirmed as harbouring brown dwarf companions. PHL 5038B has been confirmed spectroscopically (Chapter 5), and the substellar status of secondary to SDSS $1212+013$ is supported by a number of arguments (Farihi et al. 2008). However, due to its high proper motion, it was determined that the source of the near-infrared excess to NLTT 5306 is directly associated with the star, and not due to a background object (Section 2.5.1). Therefore, it will be assumed that at least 3 of the detections in this survey are confirmed white dwarf + brown dwarf binary systems containing L-dwarf secondaries. Therefore, the confirmed brown dwarfs can be used to set a lower limit on the unresolved L-dwarf and brown dwarf companion fractions.

Table 6.2 gives the percentage of the sample sensitive to the detection of L-dwarfs equal to or earlier than the specified spectral type, and the number of each spectral type potentially detected in this survey. In order to calculate the white dwarf + L-dwarf binary fraction, the effective number of detections is going to be determined. This value represents the number of each spectral type detected if the survey were $100 \%$ efficient at detecting companions of all spectral types. The effective number of detections can then be calculated by dividing the actual number of detections by the sensitivity to each spectral type. For example, if the survey sensitivity to companions of spectral types earlier or equal to L0 was $50 \%$, and $1 \mathrm{LO}$ was detected, then the effective number of detections is 2. 
This is then summed for all spectral types to give the total effective number of L-dwarfs detected:

$$
n_{\mathrm{eff}}=\frac{1}{0.98}+\frac{1}{0.98}+\frac{0}{0.97}+\frac{0}{0.96}+\frac{1}{0.95}+\frac{2}{0.91}+\frac{0}{0.86}+\frac{1}{0.82}+\frac{2}{0.74}+\frac{0}{0.65}=9.2
$$

This gives the effective number of L-dwarfs detected in the entire sample of 628 DA white dwarfs assuming a $100 \%$ sensitivity to all spectral types. The L-dwarf companion fraction is then the effective number of detections divided by the sample total. An error can be estimated by taking the square root of the reciprocal number of detections. Thus, an upper limit to the fraction of DA white dwarfs with unresolved L-dwarf companions is $f_{\mathrm{WD}+\mathrm{dL}} \leq 1.5 \pm 0.6 \%$. A lower limit can be estimated by assuming only 3 candidates (PHL 5038, SDSS $1212+013$ and NLTT 5306) are real. This gives a final range of $0.5 \leq$ $f_{\mathrm{WD}+\mathrm{dL}} \leq 1.5 \pm 0.6 \%$.

Table 6.3 gives the percentage of the sample sensitive to the detection of T-dwarfs equal to or earlier than the specified spectral type, and the number of each spectral type potentially detected in this survey. An upper limit to the fraction of white dwarf with T-dwarf companions is then calculated as detailed previously giving $f_{\mathrm{WD}+\mathrm{dT}} \leq 0.6 \pm 0.6 \%$. Clearly, this is an under-estimate in the negative direction, due to only 1 candidate $\mathrm{T}$ dwarf being detected, and there is undoubtedly not a complete absence of white dwarf + T-dwarf binaries in the universe. An average over the entire T-dwarf spectral range is a good start under these circumstances but ultimately, a more complex Bayesian analy- 
sis would yield a better result. Additional candidates from future surveys with greater sensitivity and a larger search field will also greatly improve the statistics (Chapter 7).

In order to determine the fraction of white dwarfs with brown dwarf companions, an estimate of the spectral type where the secondary becomes truly substellar must first be made. This depends on the age of the secondary, which can be estimated using the total age (main-sequence lifetime + cooling age) of the white dwarf primary. The average age of the white dwarf sample is $1.8 \pm 0.7$ Gyr. After $\approx 0.1$ Gyr brown dwarfs cool very slowly, so the average sample age can be used to estimate a spectral type where the stellar/substellar borderline occurs. Taking the upper limit on brown dwarf mass as $70 M_{\mathrm{J}}$, then an upper limit for the brown dwarf spectral type can be taken as L4 (Figure 2.25).

Table 6.4 gives the percentage of the sample sensitive to the detection of brown dwarfs equal to or earlier than the specified spectral type, and the number of each spectral type detected in this survey from L4 to T8. An upper limit to the fraction of white dwarfs with brown dwarf companions is then calculated as detailed previously giving $f_{\mathrm{WD}+\mathrm{BD}} \leq$ $1.8 \pm 0.7 \%$. A lower limit can be estimated by assuming only 3 candidates (PHL 5038, SDSS $1212+013$ and NLTT 5306) are real. This gives a final range of $0.5 \leq f_{\mathrm{WD}+\mathrm{BD}} \leq$ $1.8 \pm 0.7 \%$

It must be conceded that at the level of a single spectral sub-type (Table 6.2) such statistics will not be accurate as the low number of individual detections means there will be significant errors due to small number statistics. For each sub-type, only 0,1 or 2 candidates have been detected. Zero detections implies that no such spectral sub-type 
exists as a companion to a DA white dwarf, which is probably not the case. Neither does 1 or 2 detections necessarily give a true representation of the companion fraction at this level. However, the calculation must be broken down into individual sub-types in order to account for the drop in survey sensitivity from L0 to L9, and the respective spectral ranges for T-dwarfs and brown dwarfs (Figure 6.2). Calculating the effective number of detections for each sub-type, and combining these numbers across the whole spectral class achieve this. Thus, the sub-types with zero detections, and the errors on the spectral types of candidate secondaries (which create a range of three spectral types) will be accounted for and effectively averaged out over each spectral range. Although this does not increase the number of detections by a large factor ( 7 detections or 9.2 effective detections for L-dwarfs), Poissonian statistics can now be applied allowing an estimate of the error by taking the square root.

Although these statistics are not particularly robust, they are suitable as a first approximation using the small numbers available. In order to improve upon these numbers, the sample needs to be enlarged. In the first instance, this will be done by UKIDSS, which is set to be completed by 2012. Looking ahead, future infrared surveys with VISTA and WISE will add to the white dwarf + ultracool companion sample significantly (Chapter 7).

It should also be noted that the calculations presented here might even underestimate the total fraction of white dwarfs with ultracool companions, as this search does not include widely orbiting, resolved companions. Searches for these systems are already being 
undertaken (Day-Jones et al. 2008). However, due to the aperture size used in analysing UKIDSS photometry (2"), a small number of such systems were found as part of this work. This is due to a measurement made as part of the UKIDSS analysis called the mergedClass statistic. As noted previously, the UKIDSS mergedClass statistic provides an indication of whether a source is a point or extended source (Section 2.7). In the first instance this value can be used to remove contaminating galaxies from a search, but it can also be an indication that a system is partially resolved (e.g. PHL 5038; Chapter 5). Thus, for this survey resolved companions have only been discovered if they happen to be partially resolved in the UKIDSS database. 
Table 6.2. Actual number and effective number of detections for DA white dwarf $+\mathrm{dL}$ binaries in the UKIDSS DR5 sample based on sensitivity estimates.

\begin{tabular}{cccccc}
\hline $\begin{array}{c}\text { Spectral } \\
\text { Type }\end{array}$ & $\begin{array}{c}\text { No. Isolated WDs } \\
\text { Sensitive to Spectral } \\
\text { Type or Earlier }\end{array}$ & $\begin{array}{c}\text { \% Detectable of } \\
\text { Spectral Type } \\
\text { or Earlier }\end{array}$ & $\begin{array}{c}\text { Actual No. } \\
\text { Candidates } \\
\text { Detected }\end{array}$ & $\begin{array}{c}\text { Effective No. } \\
\text { Detected }\end{array}$ & Sample \% \\
\hline \hline L0 & 400 & $98 \%$ & 1 & 1.0 & $0.2 \%$ \\
L1 & 400 & $98 \%$ & 1 & 1.0 & $0.2 \%$ \\
L2 & 394 & $97 \%$ & 0 & 0.0 & $0.0 \%$ \\
L3 & 390 & $96 \%$ & 0 & 0.0 & $0.0 \%$ \\
L4 & 385 & $95 \%$ & 1 & 1.1 & $0.2 \%$ \\
L5 & 371 & $91 \%$ & 2 & 2.2 & $0.4 \%$ \\
L6 & 351 & $86 \%$ & 0 & 0.0 & $0.0 \%$ \\
L7 & 332 & $82 \%$ & 1 & 1.2 & $0.2 \%$ \\
L8 & 301 & $74 \%$ & 2 & 2.7 & $0.4 \%$ \\
L9 & 266 & $65 \%$ & 0 & 0.0 & $0.0 \%$ \\
\hline
\end{tabular}


Table 6.3. Actual number and effective number of detections for DA white dwarf $+\mathrm{dT}$ binaries in the UKIDSS DR5 sample based on sensitivity estimates. No candidate companions later than spectral type T3 were detected.

\begin{tabular}{cccccc}
\hline $\begin{array}{c}\text { Spectral } \\
\text { Type }\end{array}$ & $\begin{array}{c}\text { No. Isolated WDs } \\
\text { Sensitive to Spectral } \\
\text { Type or Earlier }\end{array}$ & $\begin{array}{c}\text { \% Detectable of } \\
\text { Spectral Type } \\
\text { or Earlier }\end{array}$ & $\begin{array}{c}\text { Actual No. } \\
\text { Candidates } \\
\text { Detected }\end{array}$ & $\begin{array}{c}\text { Effective No. } \\
\text { Detected }\end{array}$ & Sample \% \\
\hline \hline T0 & 226 & $56 \%$ & 0 & 0.0 & $0.0 \%$ \\
T1 & 188 & $46 \%$ & 0 & 0.0 & $0.0 \%$ \\
T2 & 152 & $37 \%$ & 0 & 0.0 & $0.0 \%$ \\
T3 & 110 & $27 \%$ & 1 & 3.7 & $0.6 \%$ \\
T4 & 90 & $22 \%$ & 0 & 0.0 & $0.0 \%$ \\
T5 & 65 & $16 \%$ & 0 & 0.0 & $0.0 \%$ \\
T6 & 44 & $11 \%$ & 0 & 0.0 & $0.0 \%$ \\
T7 & 30 & $5 \%$ & 0 & 0.0 & $0.0 \%$ \\
T8 & 16 & $4 \%$ & 0 & 0.0 & $0.0 \%$ \\
\hline
\end{tabular}


Table 6.4. Actual number and effective number of detections for DA white dwarf + brown dwarf binaries in the UKIDSS DR5 sample based on sensitivity estimates. No candidate companions later than spectral type T3 were detected.

\begin{tabular}{cccccc}
\hline $\begin{array}{c}\text { Spectral } \\
\text { Type }\end{array}$ & $\begin{array}{c}\text { No. Isolated WDs } \\
\text { Sensitive to Spectral } \\
\text { Type or Earlier }\end{array}$ & $\begin{array}{c}\text { \% Detectable of } \\
\text { Spectral Type } \\
\text { or Earlier }\end{array}$ & $\begin{array}{c}\text { Actual No. } \\
\text { Candidates } \\
\text { Detected }\end{array}$ & $\begin{array}{c}\text { Effective No. } \\
\text { Detected }\end{array}$ & Sample \% \\
\hline \hline L4 & 385 & $95 \%$ & 1 & 1.1 & $0.2 \%$ \\
L5 & 371 & $91 \%$ & 2 & 2.2 & $0.4 \%$ \\
L6 & 351 & $86 \%$ & 0 & 0.0 & $0.0 \%$ \\
L7 & 332 & $82 \%$ & 1 & 1.2 & $0.2 \%$ \\
L8 & 301 & $74 \%$ & 2 & 2.7 & $0.4 \%$ \\
L9 & 266 & $65 \%$ & 0 & 0.0 & $0.0 \%$ \\
T0 & 226 & $56 \%$ & 0 & 0.0 & $0.0 \%$ \\
T1 & 188 & $46 \%$ & 0 & 0.0 & $0.0 \%$ \\
T2 & 152 & $37 \%$ & 0 & 0.0 & $0.0 \%$ \\
T3 & 110 & $27 \%$ & 1 & 3.7 & $0.6 \%$ \\
T4 & 90 & $22 \%$ & 0 & 0.0 & $0.0 \%$ \\
T5 & 65 & $16 \%$ & 0 & 0.0 & $0.0 \%$ \\
T6 & 44 & $11 \%$ & 0 & 0.0 & $0.0 \%$ \\
T7 & 30 & $5 \%$ & 0 & 0.0 & $0.0 \%$ \\
T8 & 16 & $4 \%$ & 0 & 0.0 & $0.0 \%$ \\
\hline
\end{tabular}




\subsection{Comparison to Previous Estimates}

\subsubsection{The White Dwarf + L-Dwarf Binary Fraction}

Farihi et al. (2005) (hereafter FBZ05) estimated the white dwarf + L-dwarf binary fraction as $f_{\mathrm{WD}+\mathrm{dL}} \leq 0.5 \%$ from a near-infrared study of 371 white dwarfs with $2 \mathrm{MASS}$ photometry. 2MASS was the only source of data available to FBZ05 to estimate the unresolved companion fraction for white dwarfs. FBZ05 estimated their average sensitive to unresolved companions to be L8, the same as the peak of the sensitivity in the UKIDSS data. This is a somewhat surprising result as UKIDSS is $\approx 3$ magnitudes deeper than 2MASS. In order for the L-dwarf fraction from UKIDSS $\left(0.5 \leq f_{\mathrm{WD}+\mathrm{dL}} \leq 1.5 \pm 0.6 \%\right)$ to agree with the FBZ05 result either (1) All of the remaining photometric candidates presented here are not $\mathrm{WD}+\mathrm{dL}$ binaries i.e. the lower limit of the calculated L-dwarf fraction is true, or (2) FBZ05 have under-estimated the fraction of white dwarfs with L-dwarfs. As the first candidate for which spectroscopy was obtained (PHL 5038, Chapter 5) was confirmed to be real, and in other cases the photometric classification remains quite convincing, then explanation (1) seems less likely than (2). To investigate this further, a detailed examination will be made as to how FBZ05 arrived at their L-dwarf companion fraction. The same method will then be followed for the UKIDSS sample, and a comparison made to the detailed sensitivity analysis discussed previously.

It should be noted that the FBZ05 sample includes white dwarfs not of spectral type DA. However, these are a minority in the sample, and their removal would not significantly 
affect the fraction calculated by FBZ05.

Figures 6.5 and 6.6 show the distance distributions of both surveys, with the mean distances plotted $($ FBZ05 $=57 \mathrm{pc}$ and UKIDSS $=144 \mathrm{pc})$. FBZ05 calculate the detection limit of their survey by determining the "average" white dwarf from their sample (found by calculating the average temperature, $T_{\text {eff }}=13000 \mathrm{~K}$, of their sample and assuming $\log g=8.0$ ), and placing an upper limit on any putative companion's spectral type assuming this "average" white dwarf is situated at the mean sample distance. FBZ05's "average" white dwarf has absolute magnitudes $M_{H}=11.8$ mags and $M_{K_{\mathrm{s}}}=11.9$ mags. These magnitudes are beyond the detection limits of the 2MASS survey $\left(M_{H} \sim 11.6\right.$ mags and $\left.K_{\mathrm{s}} \sim 10.8 \mathrm{mags}\right)$, and so FBZ05 set the detection limit by assuming a companion has a magnitude equal to the difference between the 2MASS survey limits and this "average" white dwarf. This gives a limit of L8 and L4 in the 2MASS $H$ - and $K_{\mathrm{s}}$-bands respectively.

Following the FBZ05 method, an "average" white dwarf must be analysed using the UKIDSS sample, for which the average temperature is $T_{\text {eff }}=14000 \mathrm{~K}$ (Figure 6.7). Assuming a surface gravity of $\log g=8.0$ this equates to 2MASS absolute magnitudes of $M_{H}=11.7$ mags and $M_{K_{\mathrm{s}}}=11.8$ mags. Using the average distance of $144 \mathrm{pc}$, the $5 \sigma$ limits of the UKIDSS survey are $M_{H}=13.0$ mags and $M_{K_{\mathrm{s}}}=12.6$ mags.

It should be noted at this point that these are fainter than the corresponding magnitudes of the "average" white dwarf, differing from the FBZ05 analysis. In FBZ05 the "average" white dwarf is in fact brighter in magnitude than the 2MASS limits, allowing for a limit 


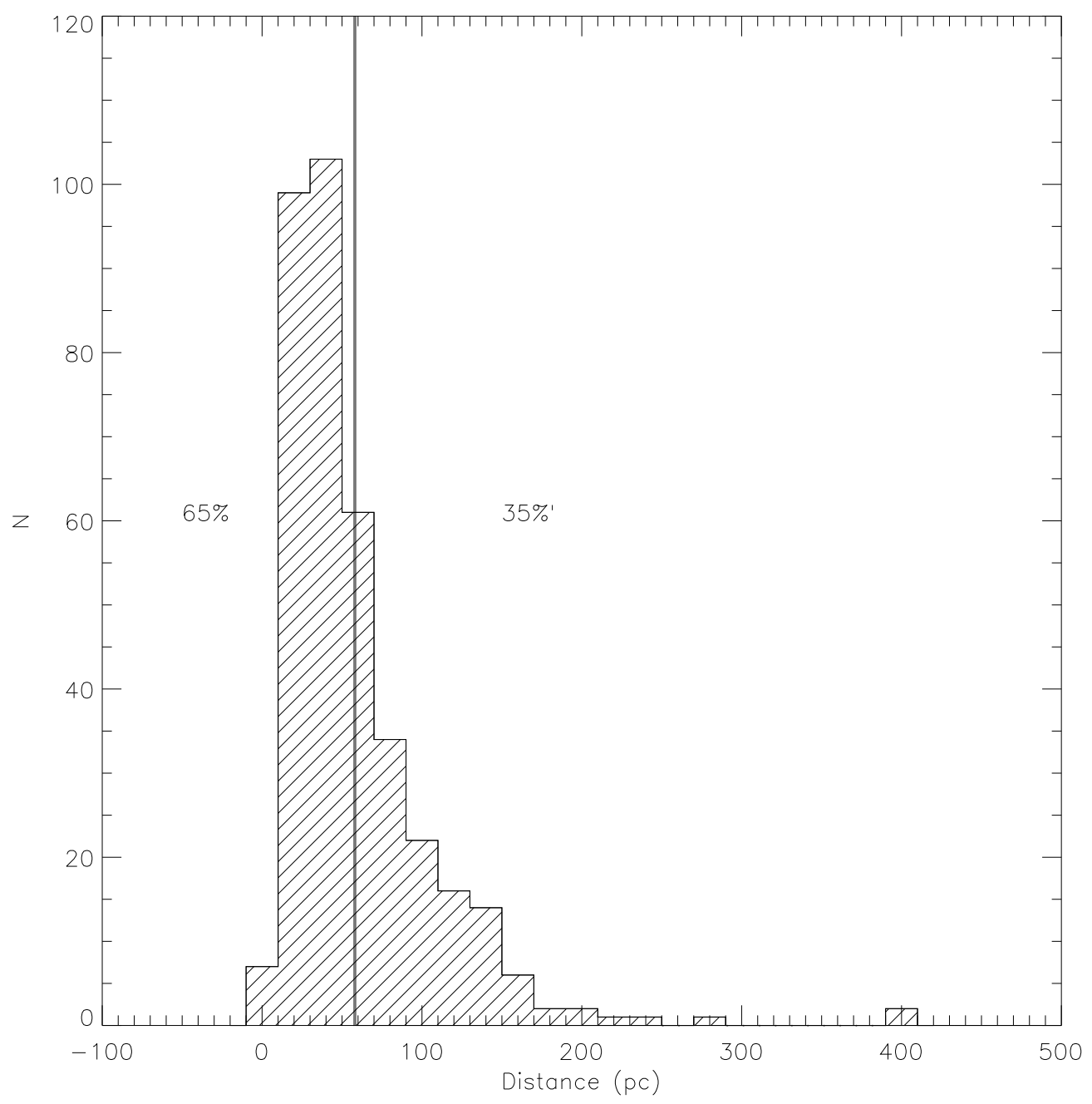

FIGURE 6.5. Distance distribution of the white dwarfs presented in Farihi et al. (2005). The mean distance is shown as a solid line. 


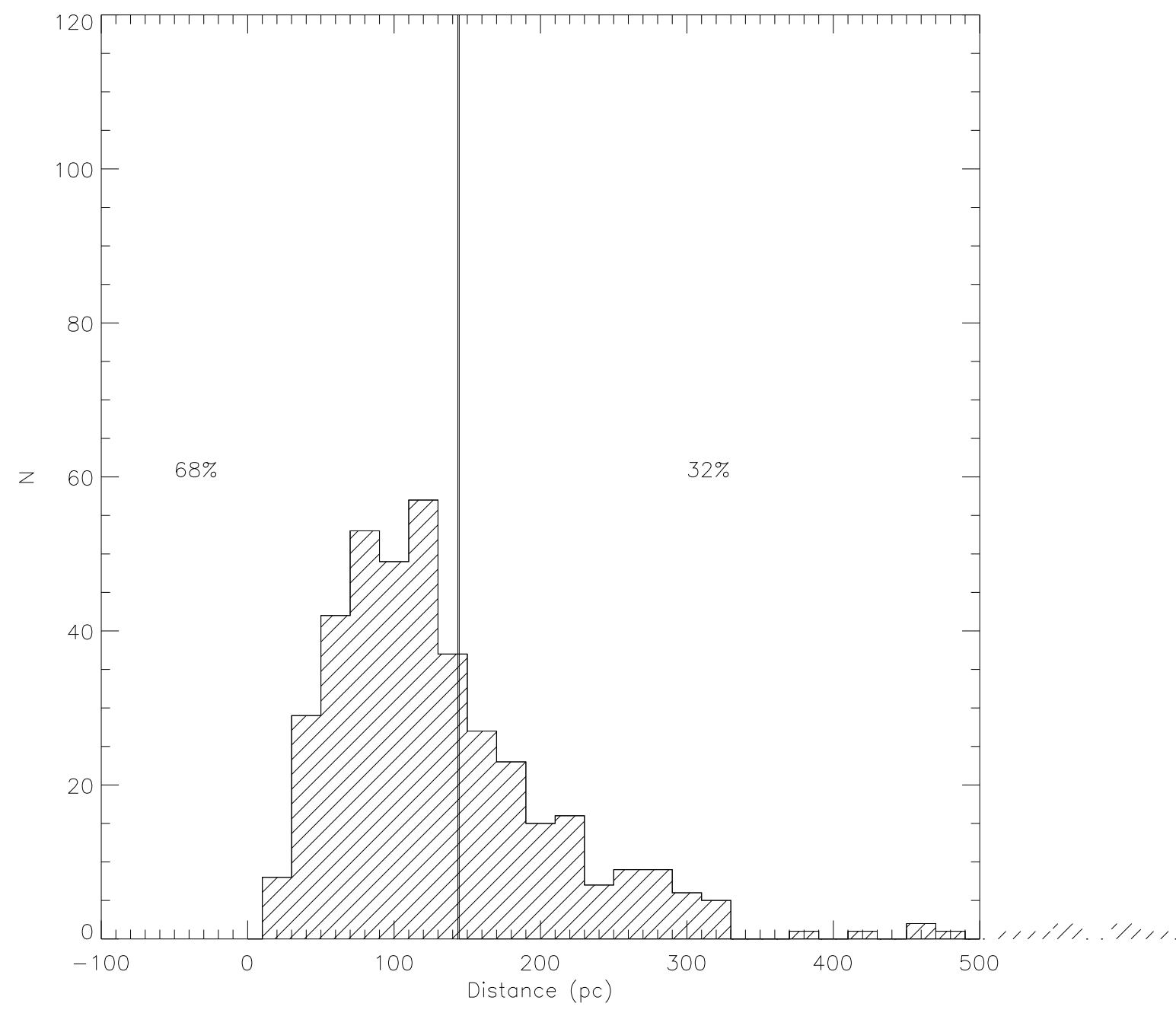

FIGURE 6.6. Distance distribution of the single DA white dwarfs present in the UKIDSS sample. The mean distance is shown as a solid line. 
to be set on the minimum luminosity of a putative companion by using the difference between those two values. As the opposite case is true for UKIDSS, it would make sense to use the addition of a typical $3 \sigma$ error as the limiting magnitude. An interesting point can be made here; if FBZ05 had added the $3 \sigma$ error at the 2MASS limit to their initial estimate, then the "average" companion would have been brighter, and therefore of an earlier spectral type.

At $144 \mathrm{pc}$ the "average" UKIDSS white dwarf has magnitudes $H=17.5$ mags and $K=17.6$ mags, which have typical errors of \pm 0.05 and \pm 0.1 respectively. Assuming a companion is detected at the $>3 \sigma$ level, this corresponds to a companion with apparent magnitudes of $H=20.0$ mags and $K=18.8$ mags. This sets a limiting spectral type for the UKIDSS survey of later than T9 in both bands.

Figures 6.1 and 6.2 shows that it is indeed the case that the UKIDSS survey is sensitive to late (>T8) T-dwarf companions. However, the sensitivity analysis for each white dwarf individually shows that this accounts for $<2 \%$ of the entire sample. Clearly it is not the case that this survey is capable of detecting late T-dwarf companions around every white dwarf. Indeed, it was shown in Section 6.3 that the sensitivity of this survey peaks at the L-T border. The UKIDSS "average" white dwarf is clearly not representative of either the mean or the median star in the sample. Indeed, it is only representative of the extreme of the survey sensitivity. Therefore, it is suspected that FBZ05's "average" white dwarf is unlikely to represent their mean or median star either. For example, if the distance distribution to each sample is inspected, $35 \%$ and $32 \%$ of white dwarfs lie beyond the 
average distance from FBZ05 and UKIDSS respectively. Hence, it is likely that at least that fraction are not in fact as sensitive to the presence of low mass companions as the "average" star.

FBZ05 state "near-infrared excess detection requires photometric accuracy, not deep imaging". In fact, as UKIDSS is $\approx 3$ magnitudes deeper than 2MASS, the UKIDSS survey is more accurate at the fainter end of the 2MASS survey where many of the white dwarfs lie (Figure 6.5). In 2MASS the photometric signal-to-noise ratio is $>10$ for $K_{S}<14.3$ mags, so a typical error is 0.1 mags. In the equivalent UKIDSS bandpass, the typical error is 0.005 mags. Clearly, UKIDSS will provide much better accuracy at the 2MASS limits, and continue this trend until the limits of the survey are reached. Indeed, it may be the case that 2MASS is not as sensitive as the "average" white dwarf would suggest.

Clearly, the use of an "average" white dwarf to place sensitivity limits on a sample may be the problem. The detection of an ultracool companion requires that the companion exceed the white dwarf's luminosity by $3 \sigma$ at an infrared wavelength. The luminosity of a star is proportional to $R^{2}$ and $T^{4}$, hence an "average" white dwarf determined according to temperature is not representative of the sample. Unfortunately, FBZ05 do not detail the parameters needed for each white dwarf (Effective temperature and distance) to perform a similar analysis to that presented for the UKIDSS data. The reasons for the surprisingly similar claimed sensitivities of both the 2MASS and UKIDSS surveys cannot be fully understood until such an analysis can be performed. 


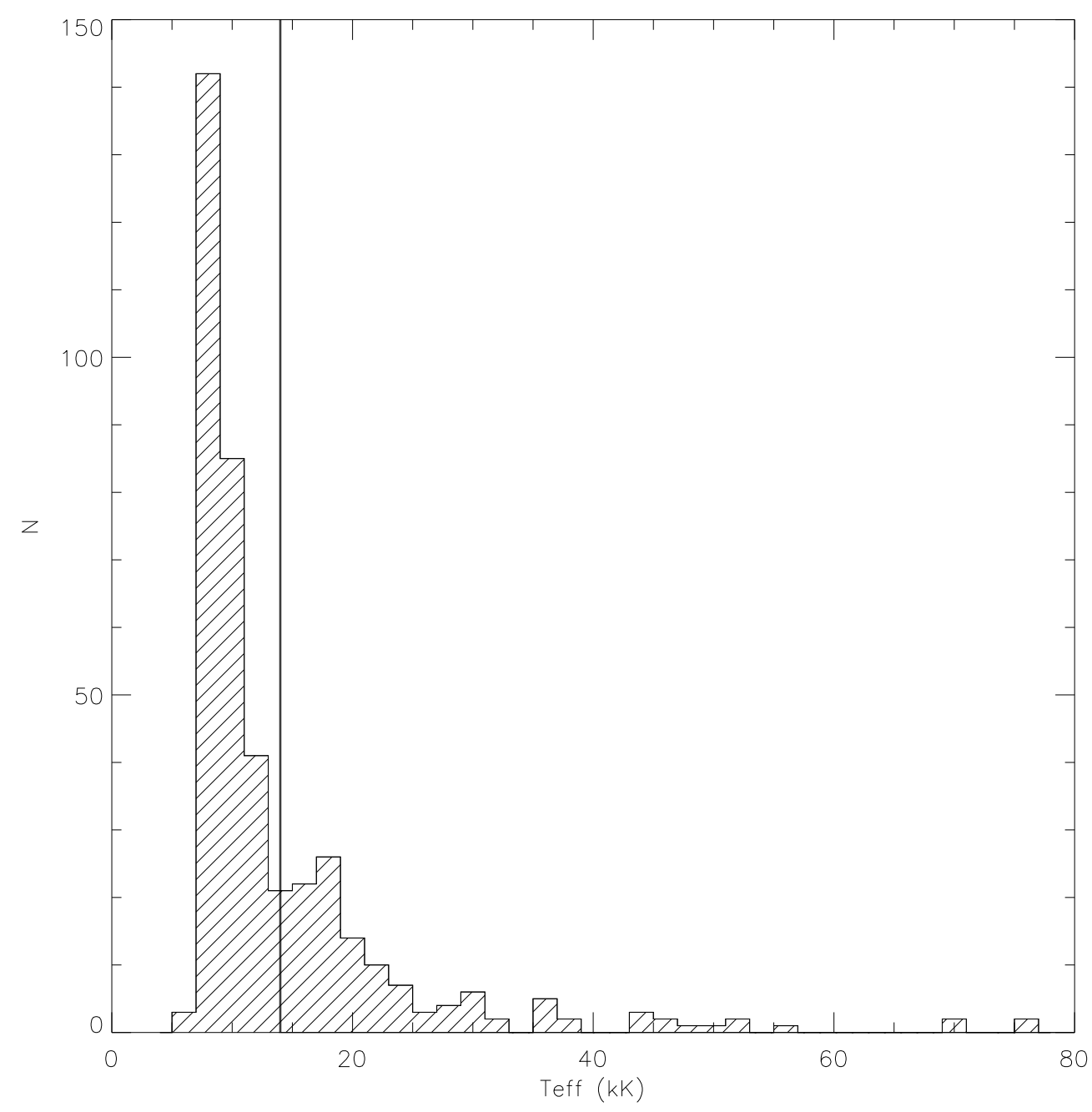

FIGURE 6.7. Temperature distribution of the single DA white dwarfs present in the UKIDSS sample. The mean temperature is shown as a solid line. 


\subsubsection{The Main-sequence Star + Brown Dwarf Binary Fraction}

McCarthy \& Zuckerman (2004) presented a coronographic near-infrared search for substellar companions to nearby main-sequence stars. They found that the frequency of brown dwarf companions to $\mathrm{G}, \mathrm{K}$ and $\mathrm{M}$ stars orbiting at a distance between 75-300 AU to be $1 \pm 1 \%$. They also calculated a companion fraction for massive brown dwarfs orbiting between $120-1200 \mathrm{AU}$ to be $0.7 \pm 0.7 \%$. Grether \& Lineweaver (2006) analysed the relative fraction of close $(\mathrm{P}<5 \mathrm{yr})$ stellar, brown dwarf and planetary companions to nearby sun-like stars and found that close brown dwarf companions occur at a frequency of $<1 \%$. Therefore, the brown dwarf companion fraction to white dwarfs $\left(0.5 \leq f_{\mathrm{WD}+\mathrm{BD}} \leq 1.8 \pm 0.7 \%\right)$ and hence, their progenitors, from the UKIDSS survey agrees with the estimates of both McCarthy \& Zuckerman (2004) and Grether \& Lineweaver (2006) within errors.

However, there exists a bias among white dwarfs in that they are generally descended from stars more massive than the Sun. It could easily be the case that such stars have a greater frequency of brown dwarf companions than solar-type stars.

The brown dwarf desert is a noted deficit in the frequency of brown dwarf companions relative to the frequency of less massive planetary companions (Marcy \& Butler 2000) or relative to the frequency of more massive stellar companions to Solar-like hosts. Grether \& Lineweaver (2006) verified the existence of this phenomenon, with the desert existing at close separations $(<3 \mathrm{AU})$. Determining the separations of the $\mathrm{WD}+\mathrm{BD}$ binaries in 
the UKIDSS sample will allow additional investigation of the brown dwarf desert. It may even be possible to determine the companion fraction at different orbital radii.

\subsection{Summary}

Limits have been placed on the latest spectral type of unresolved companion detectable around each of the apparently single DA white dwarfs detected in the UKIDSS database. It was found that this survey has a sensitivity of $\approx 98 \%$ to companions of spectral type L0 or earlier and $\approx 56 \%$ to companions of spectral type T0 or earlier. These sensitivities were used in conjunction with the photometrically detected L and T-dwarf companions to estimate the unresolved brown dwarf companion fraction to DA white dwarfs as $0.5 \leq$ $f_{\mathrm{WD}+\mathrm{BD}} \leq 1.8 \pm 0.7 \%$.

If the majority of the L-dwarf photometric candidates are confirmed, then the L-dwarf companion fraction $\left(f_{\mathrm{WD}+\mathrm{dL}} \leq 1.5 \%\right.$ if all confirmed $)$ will be significantly higher than that of Farihi et al. (2005). Once possible explanation for this discrepancy is that Farihi et al. (2005) have used an "average" white dwarf to set the limits of their 2MASS survey. By utilising the same method for the UKIDSS white dwarfs, it was shown that this "average" white dwarf may not be representative of the mean survey sensitivity. A more thorough analysis of the 2MASS survey sensitivity is required, using the same method employed here, before the differences can be fully understood. This will require each white dwarf to be analysed individually in order to place a limit on the coolest detectable companion around each star. 
The fraction of ultracool companions to white dwarfs was found to be consistent with estimates of the main-sequence + brown dwarf binary fraction. 


\section{Chapter 7}

\section{Summary and Future Work}

\subsection{Overview}

In this thesis I have undertaken a near-infrared search for ultracool companions to white dwarfs with the ultimate aim of further constraining the white dwarf + L-dwarf/T-dwarf/brown dwarf binary fractions. This final chapter summarises the key results and conclusions presented in this thesis and propose future work to improve the statistics with new surveys.

\subsection{Summary of Results}

In Chapter 2 the UKIDSS LAS DR5 was cross correlated with catalogues of spectroscopically identified white dwarfs, in order to search for stars displaying near-infrared excesses indicative of low mass companions or debris disks. The sample included 639 hydrogen atmosphere DA white dwarfs with $1500<T_{\text {eff }}<100,000 \mathrm{~K}$ and $6.5<\log g<$ 
9.5, and 10 helium atmosphere DB white dwarfs with $3500<T_{\text {eff }}<30,000 \mathrm{~K}$ and $7.0<\log g<9.0$, all with both $H$ and $K$-band photometry present in the UKIDSS archive. 235 white dwarfs were identified with near-infrared excesses, with 206 of these recovering previously identified $\mathrm{DA}+\mathrm{M}$ and $\mathrm{DB}+\mathrm{M}$ type systems. Of the 29 remaining candidates, 24 had excesses in multiple filters indicative of a low mass companion, with seven of these having a predicted mass in the range associated with brown dwarfs, and a further 13 likely very low mass stellar companions. Two white dwarfs were identified with putative companions that are likely spectral class K. Four of the sample showed evidence of contamination by a foreground or background object. The remaining three each had a $K$-band excess indicative of a debris disk including the previously discovered DAd SDSS J1228+104 (Gänsicke et al. 2006). Two magnetic white dwarfs were found with a near-infrared excess; (1) SDSS J1212+01 - a previously identified DAH+dL8 binary (Debes et al. 2006, Koen \& Maxted 2006, Burleigh et al. 2006b, Farihi et al. 2008), and (2) SDSS J1250+154 - potentially explained by the presence of an M8 companion and additional cyclotron emission.

In Chapter 3 eleven DA white dwarfs were observed, all of which have been identified as having M-type companions, The secondary in each system was assigned a spectral type in the near-infrared by comparison of key features in the star's spectrum in comparison to existing spectra of M-dwarfs. Six of the secondary star classifications agreed with previous spectral typing in the optical and near-infrared. However, all of the spectroscopic results are in good agreement with photometry to \pm 1 spectral type. The slight differences might be due to errors in distance estimates or activity intrinsic to the companion. The 
accuracy of the spectral types were tested by measuring the equivalent width of the $\mathrm{NaI}$ line at $2.2 \mu \mathrm{m}$, with no anomalies found. All of the systems had near-infrared colours expected of white dwarf $+M$-dwarf binary systems. In systems where the companion is known to have a short period or the period is currently unknown no evidence was found for accreted material during a common envelope phase.

In Chapter 4 the spectral type of the low mass companion to PG 1234+482 was estimated using $H$ and $K$-band spectroscopy obtained from the WHT LIRIS instrument and Spitzer IRAC $4.5 \mu \mathrm{m}$ and $8.0 \mu \mathrm{m}$ photometry. Due to uncertainty in the 2MASS photometry used to place these data on an absolute flux scale, an estimated spectral type of L $0 \pm 1$ (M9-L1) was assigned. The total age of the system is $\approx 1 \mathrm{Gyr}$, where the substellar boundary spectral type is $\approx \mathrm{L} 1 \pm 1$. This makes PG 1234 the hottest and youngest $\left(\mathrm{t}_{\text {cool }} \approx\right.$ $10^{6}$ yr; Liebert, Bergeron \& Holberg 2005) DA white dwarf with a possible brown dwarf companion.

The confirmation of an ultracool companion to PG 1234 leads to the speculation that the metals in the hot white dwarf's atmosphere are at least in part being accreted from the companion's wind. To answer this a constraint is needed on the separation of the pair, by either high resolution imaging and/or radial velocity measurements. Current evidence suggests that the system has an orbital separation of $<$ few solar radii (Section 4.7). If confirmed, it is likely that the companion is a survivor of a common envelope phase.

In Chapter 5 the presence of a brown dwarf companion to the white dwarf PHL 5038A was confirmed and assigned a spectral type of L8-L9 based on the measurement of stan- 
dard spectral indices in the near-infrared, the first time this has been accomplished for a bona fide WD+BD binary. The spectral type was corroborated by photometry and by comparison with the spectrum of an L8 field brown dwarf. The system is situated $64 \mathrm{pc}$ from Earth, and the brown dwarf has a projected orbital separation from its primary of 55 AU. PHL 5038B has the potential of being used as a benchmark brown dwarf if its age can be determined more accurately (Current estimate $=1.9-2.7 \mathrm{Gyr}$ ). A measurement of a trigonometric parallax for this system would allow for a more accurate constraint on the radius and, via models, the mass of the white dwarf. This would then allow a better estimate for the total age of the system. This is required in order to accurately estimate the age of the brown dwarf companion, which is essential when modeling substellar atmospheres.

Finally, in Chapter 6 limits were placed on the latest spectral type of unresolved companion detectable around each of the apparently single DA white dwarfs detected in the UKIDSS database. It was found that this survey has a sensitivity of $\approx 98 \%$ to companions of spectral type L0 or earlier and $\approx 56 \%$ to companions of spectral type T0 or earlier.These sensitivities were used in conjunction with the photometrically detected L and T-dwarf companions to estimate the unresolved brown dwarf companion fraction to DA white dwarfs as $0.5 \leq f_{\mathrm{WD}+\mathrm{BD}} \leq 1.8 \pm 0.7 \%$. The fraction of ultracool companions to white dwarfs was found to be consistent with estimates of the main-sequence + brown dwarf binary fraction.

The results of Chapter 2 show that large, deep near-infrared surveys are indeed the ideal 
hunting ground for low mass stellar/substellar companions to white dwarfs. The use of near-infrared photometry in assigning spectral types to such companions seems to be accurate to within \pm 1 spectral types, certainly for M-dwarfs (Chapter 3), and for the spectroscopically confirmed L-dwarfs presented in this thesis (Chapters 4 and 5). However, the sensitivity analysis of Chapter 6 indicates that a much larger sample will be needed to effectively detect T-dwarf companions. Mid-infrared surveys may be more ideally suited to the detection of companions of spectral type $\mathrm{T}$ and debris disks, as a photometric excess for these objects is more likely to present at longer wavelengths.

The results of Chapter 6 imply a much larger brown dwarf companion fraction to white dwarfs than previously estimated. However, as this is consistent with the main-sequence + brown dwarf binary fraction, a higher survival rate (certainly at the high mass end of the brown dwarf mass function) may be implied for such systems.

\subsection{Future Work}

\subsubsection{Follow-up Observations}

Spectroscopic follow-up to confirm and provide a spectral type for each of the candidate systems presented in this thesis will be the initial priority. In addition, radial velocity measurements will then be taken for spectroscopically confirmed systems in order to provide an orbital separation. This is important for the detection of post-CE and pre-CV systems, and also for identifying benchmark brown dwarfs. The bimodal distribution 
of white dwarf binaries, and binary fractions at different orbital separations can also be investigated.

There are several telescopes and instruments for the purpose of spectroscopic followup in the near-infrared. Examples are Gemini + NIRI or GNIRS, and NASA's IRTF which can provide near-infrared spectra. The VLT + XSHOOTER can simultaneously take spectra in the UV, Optical and near-infrared. This would be particularly useful for constraining the white dwarfs effective temperature and surface gravity, and hence accurately determining the star's age. This is important in identifying benchmark brown dwarf systems.

\subsubsection{The Future of UKIDSS}

Since this thesis work was completed the UKIDSS LAS DR6 has been released (October 2009). DR6 represents $\approx 1400 \mathrm{deg}^{2}$ (inclusive of previous data releases) of data completed in all four UKIDSS passbands, and represents $42 \%$ of the final planned survey. In contrast DR5 represented $32 \%$ of the final planned survey.

However, due to cuts in funding and available telescope time the planned final area for the LAS has now changed (Figure 7.1; Steven Warren, private communication). The survey now consists of four blocks; (1) Block L1. This is the original LAS spring equatorial block. (2) Blocks L2a and L2b. These are areas within the original L2 block that were partially completed.(3) Block L3. The original L3 was coincident with SDSS Stripe 
82. This has undergone a change in RA distribution in order to accelerate completion, and has now expanded from $213 \mathrm{deg}^{2}$ to $1258 \mathrm{deg}^{2}$. (4) Block L4. This is anew block intended for Herschel-ATLAS support. All blocks will be observed in $Y J H K$ and the final planned area is now $3792 \mathrm{deg}^{2}, \approx 85 \%$ of the original survey area. Second epoch $J$-band imaging will comprise of $2000 \mathrm{deg}^{2}$, drawn from blocks L1, L2a and L2b.

The updated UKIDSS surveys are still expected to be completed in 2012 and this data is essential for the completion of the work that this thesis has begun. As the white dwarf + brown dwarf binary fraction is so small, it is important to increase the size of the sample significantly. This in turn will allow tighter constraints to be placed on the number of white dwarfs with detectable substellar companions, and increase the current range of spectral types. It is particularly important to increase the sample size sensitive to Tdwarfs, which currently accounts for only 226 of the DA white dwarfs in the UKIDSS sample (of which only 16 were sensitive to all spectral types equal to or earlier than T8). Follow-up radial velocity studies will then be important for investigating the proposed bimodal distribution of white dwarfs with ultracool companions, and allow for the detection of pre-CV and post-CE systems.

Assuming an even spatial distribution of white dwarfs, the sample number from UKIDSS should have more than doubled upon completion of the LAS. It should be noted that in this thesis I have used spectroscopically confirmed white dwarfs. Extending the survey to include candidate white dwarfs identified by optical colours would greatly increase the sample size. However, spectroscopic confirmation of these photometric candidates 
would then be required in both the optical and the near-infrared to confirm the presence of the white dwarf and companion respectively. The spectra are also needed to constrain the white dwarf parameters, especially distance, and hence constrain the companion's spectral type.

This survey does not take wide resolved white dwarf binaries into account, with searches for these systems already being undertaken (Day-Jones et al. 2008). The planned second epoch UKIDSS $J$-band observations will allow for the detection of such pairs. This will add to the binary statistics significantly, with the investigation of binary frequency at a wider range of orbital separations.

\subsubsection{VISTA}

The Visible and Infrared Survey Telescope for Astronomy (VISTA; Emerson et al. 2004) is a $4 \mathrm{~m}$ wide field survey telescope for the southern hemisphere. The telescope will be equipped with a near-infrared camera containing 67 million pixels of mean size 0.34 " (1.65 degree diameter) and makes use of broad band $Z, Y, J, H$ and $K_{\mathrm{s}}$ filters, and a narrow band filter at $1.18 \mu \mathrm{m}$.

Like UKIDSS, the VISTA telescope will undertake a complimentary set of 6 surveys; UltraVISTA, the VISTA Kilo-Degree Infrared Galaxy Survey (VIKING), the VISTA Magellenic Survey (VMC), VISTA Variables in Via Lactea (VVV), the VISTA Hemisphere Survey (VHS) and the VISTA Deep Extragalactic Observations Study (VIDEO).The sky 


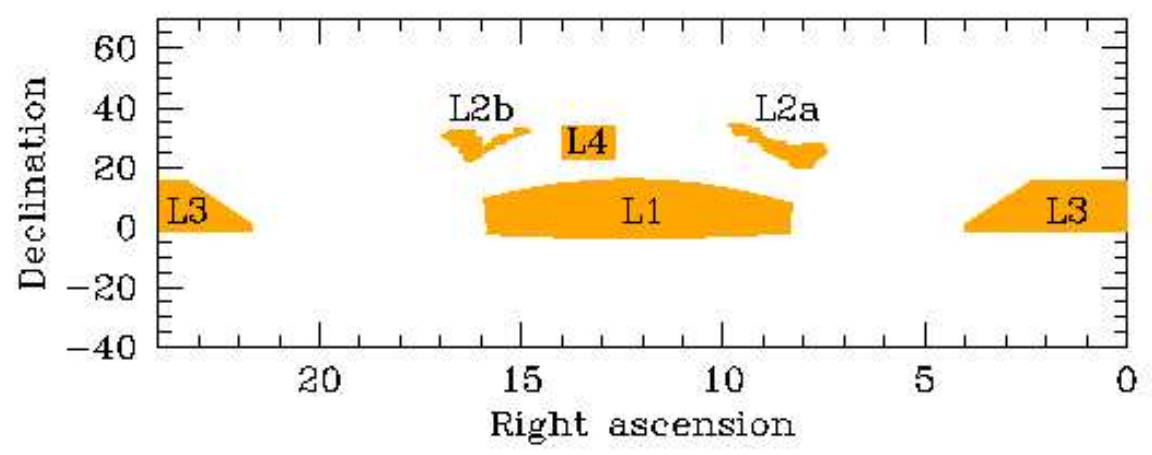

FIGURE 7.1. The revised Large Are Survey footprint. 
coverage for each of these surveys is shown in Figure 7.2. The survey of most importance for the continuation of this work is the VHS.

The VHS will image the entire $20,000 \mathrm{deg}^{2}$ of the southern sky down to limiting magnitudes of $Y=21.2, J=21.2, H=21.2$ and $K_{\mathrm{s}}=20.0$ mags. Thus, a search can be performed for low mass companions and debris disks to white dwarfs in low galactic latitudes with detection limits similar to that, and in many cases better than that, of UKIDSS. The increased area over UKIDSS should also increase the sample size significantly. In other words, VISTA will detect more white dwarfs, and be more sensitive to T-dwarf companions than UKIDSS.

However, there are very few spectroscopically identified white dwarfs in the southern hemisphere. The $2 \mathrm{dF}$ survey made some progress in this area, but only for $\approx 1000 \mathrm{DA}$ white dwarfs (Vennes et al. 2002). Rowell, Hambly \& Bergeron (2009) recently identified $\approx 9500$ photometric white dwarf candidates using the SuperCOSMOS Sky Survey, which increases the sample significantly, but has no spectroscopic follow-up. The VST surveys and the Australian Skymapper will do the equivalent of SDSS for the South, but also will not provide spectroscopic follow-up. This will make exploitation of VISTA more problematic than the methods used in this thesis. Spectroscopic confirmation of both system components will require optical and near-infrared data. This is entirely possible with second generation instruments such as XSHOOTER on the VLT (D'Odorico et al. 2006). The large amount of time needed for spectroscopic follow-up of Southern white dwarfs will, however, require significant allocations of telescope time in an in- 
creasingly competitive environment due to recent cuts in telescope access for the UK. Practically, a large number of good candidates will be required before any large scale confirmation program can be implemented.

\subsubsection{WISE}

The Wide-field Infrared Survey Explorer (WISE) satellite will provide an all sky survey in the mid-infrared from 3 to $25 \mu \mathrm{m}$. It will have a sensitivity approximately 500,000 times greater than the previous Cosmic Background Explorer (COBE) satellite, and up to 1000 times greater than the Infrared Astronomical Satellite (IRAS). WISE was launched on the $14^{\text {th }}$ December 2009.

The $5 \sigma$ point source sensitivity of WISE is shown in Figure 7.3 as well as previous or planned all-sky surveys. It can be seen that WISE should indeed be capable of detected mid-infrared excesses in all but the faintest white dwarfs from UKIDSS, and certainly from all the white dwarfs detected in 2MASS. A survey of cool white dwarfs to search for debris disks should therefore be feasible.

The WISE instrument is a four-channel imager which operates in a single mode, taking overlapping snapshots of the sky. The telescope has a $40 \mathrm{~cm}$ diameter and has $\mathrm{HgCdTe}$ and Si:As $1024 \times 1024$ detector arrays at 3.4, 4.6, 12 and $22 \mu \mathrm{m}$. WISE will have a resolution of 6", except at $22 \mu \mathrm{m}$ where it will be $12 "$. The solid-hydrogen cryostat is expected to give a mission lifetime of 10 months. 


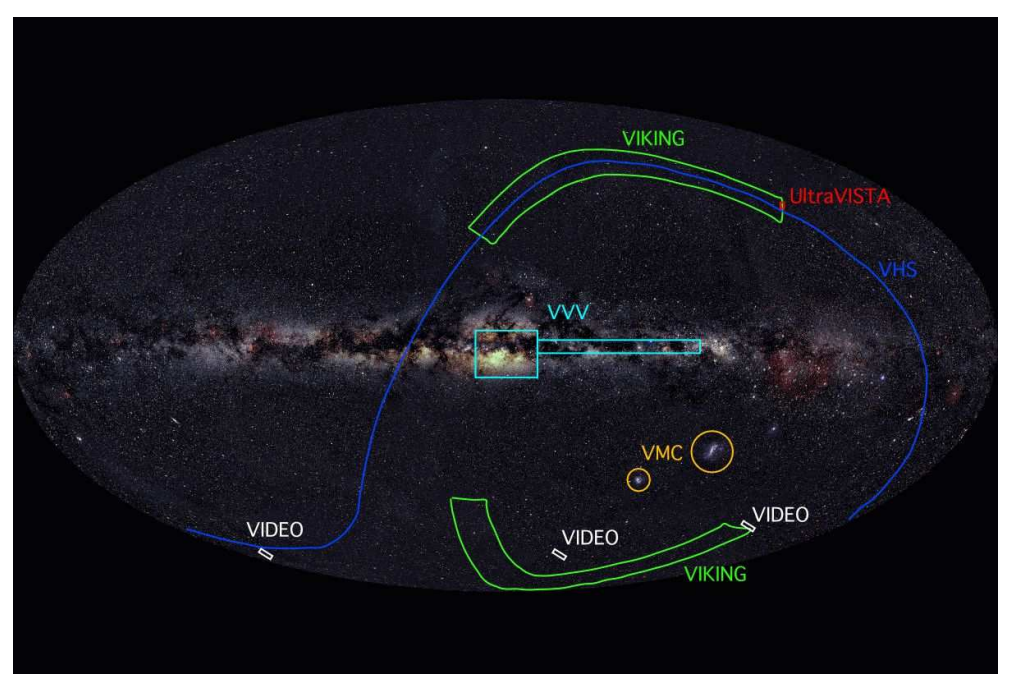

FIGURE 7.2. Sky coverage of VISTA surveys, overlaid on a 2MASS image of the whole sky. 
WISE will make a preliminary release of data taken during the first half of the survey six months after the end of on-orbit data acquisition. For the current lifetime estimate of 10 months, the first data release would be in April 2011. The final data release will be 11 months later. Therefore, all the data collected by WISE should be accessible to the astronomical community in the latter half of 2012.

WISE will be of particular importance in the photometric confirmation of debris disk candidates from the UKIDSS and VISTA surveys, aswell as providing an opportunity to continue the search for very low mass companions to white dwarfs. WISE will certainly provide the most sensitive survey for finding mid to late $\mathrm{T}$ dwarfs and potentially $\mathrm{Y}$ dwarf companions to white dwarfs. 


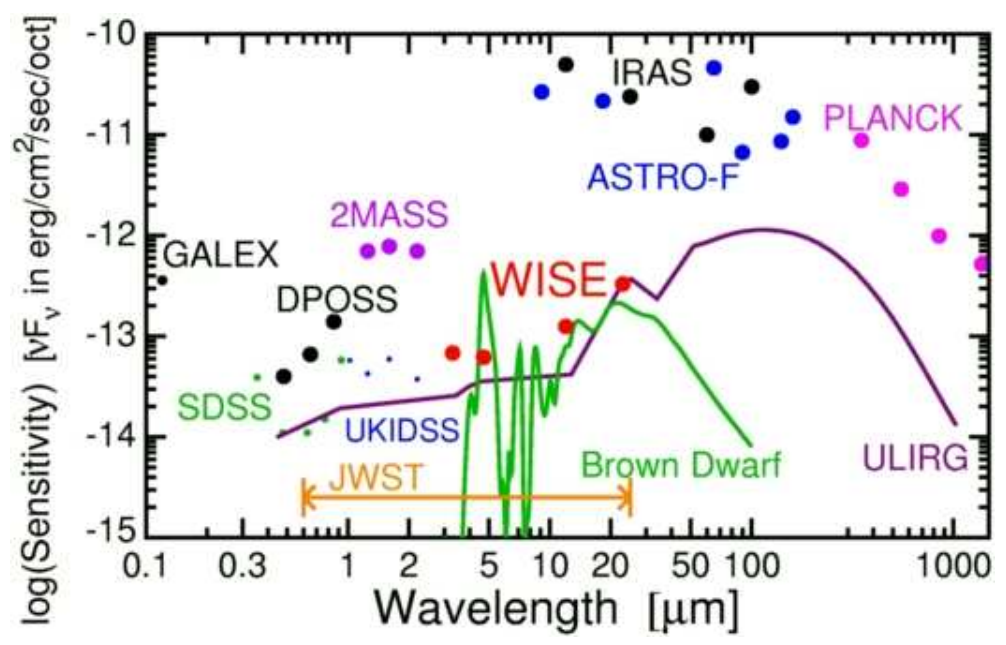

FIGURE 7.3. The $5 \sigma$ point source sensitivities of WISE and previous or planned allsky surveys. The planned wavelength range for the JWST is indicated. The dot size shows the planned sky coverage. GALEX is a small Explorer (SMEX), DPOSS is the groundbased Digital Palomar Observatory Sky Survey, ASTRO-F is the Japanese satellite, renamed Akari after launch on 22 Feb 2006, and Planck is the European

CMB mission. Figure from www.astro.ucla.edu/wright/WISE/ 
Appendix A

Chapter 2: Spectra 


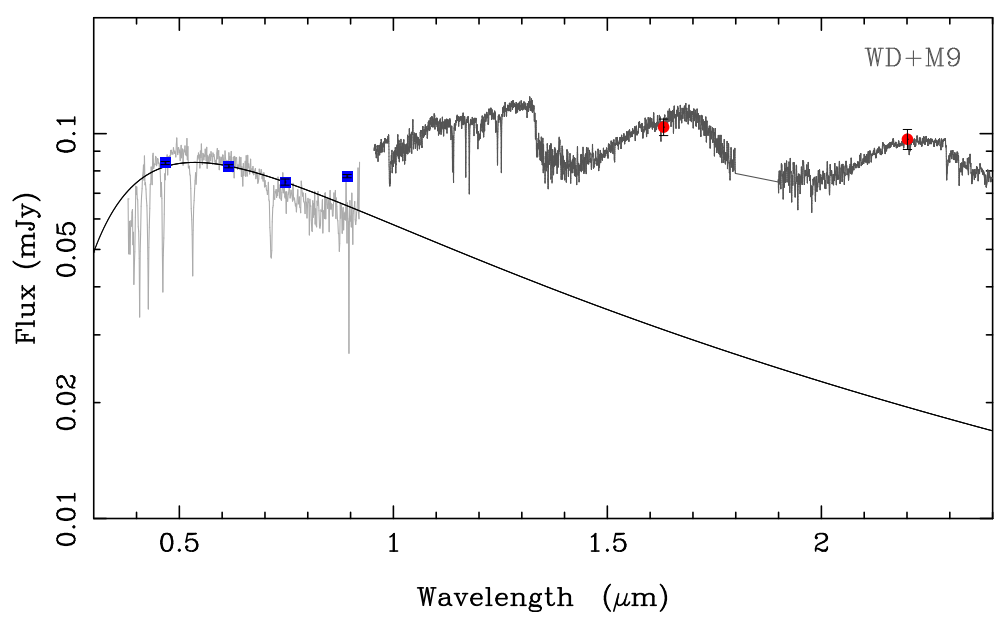

FIGURE A.1. SDSS J003923.04+003534.7 model spectrum (black solid) with SDSS $g_{r i z}$ (blue) and UKIDSS $H K$ photometry (red). Also shown are the SDSS spectrum (light grey) a composite $\mathrm{WD}+\mathrm{M} 9$ spectrum (dark grey).

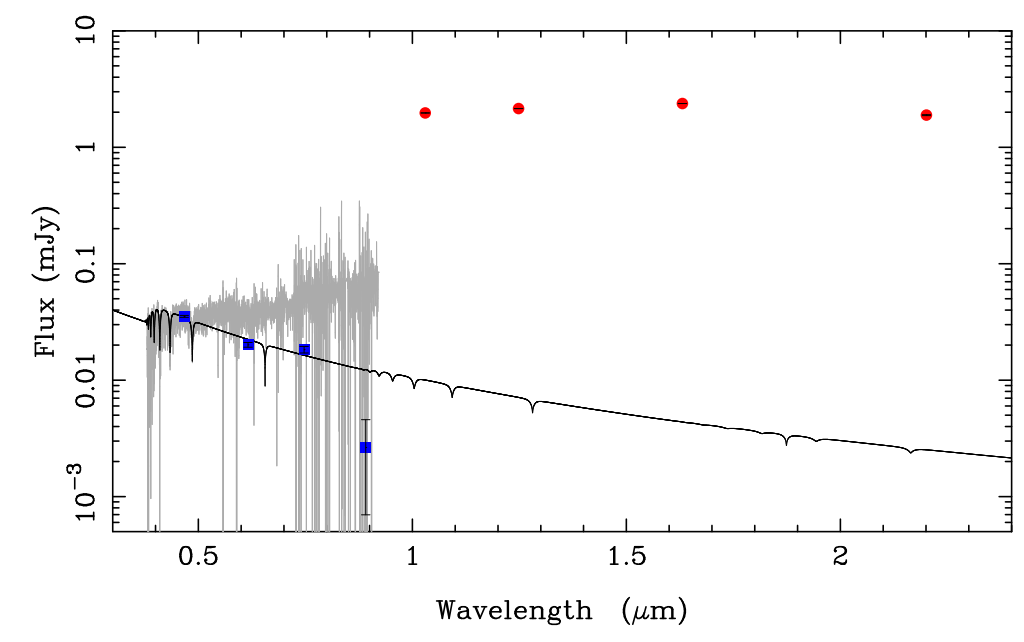

FIGURE A.2. SDSS J032317.00-002612.7 model spectrum (black solid) with SDSS $g r i z^{\prime}$ (blue) and UKIDSS $J H K$ photometry (red). The extreme near-infrared excess is likely caused by a bright background star. 


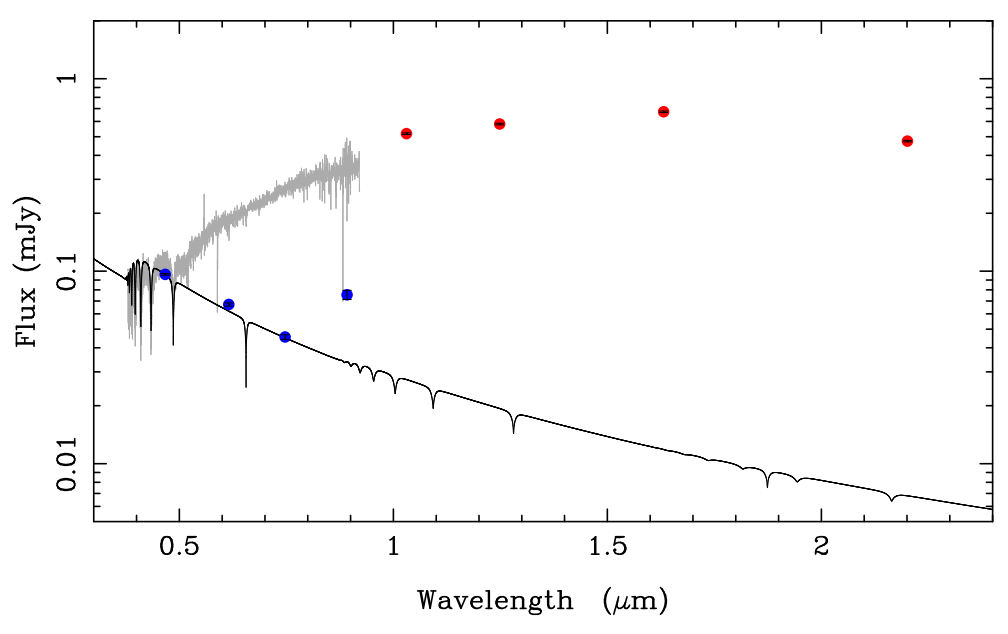

FIGURE A.3. SDSS J034221.55+005345.6 model spectrum (black solid) with SDSS griz' (blue) and UKIDSS $Y J H K$ photometry (red). The extreme nearinfrared excess is likely caused by a bright background star.

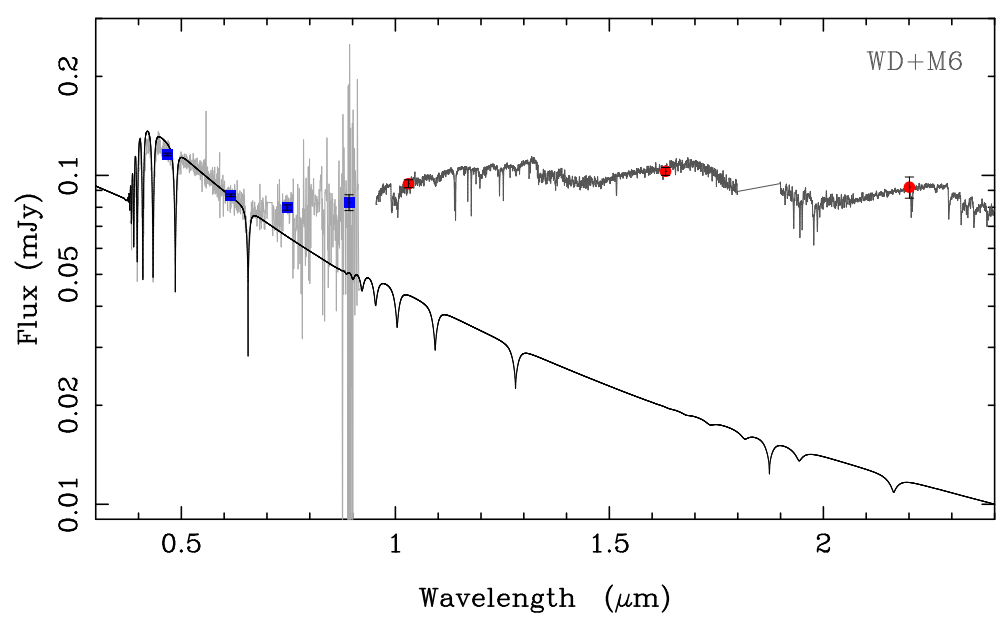

FIGURE A.4. SDSS J085956.47+082607.5 model spectrum (solid black) with SDSS griz' (blue) and UKIDSS $Y H K$ photometry (red). Also shown are the SDSS spectrum (light grey) and a composite WD+M6 spectrum (dark grey). 


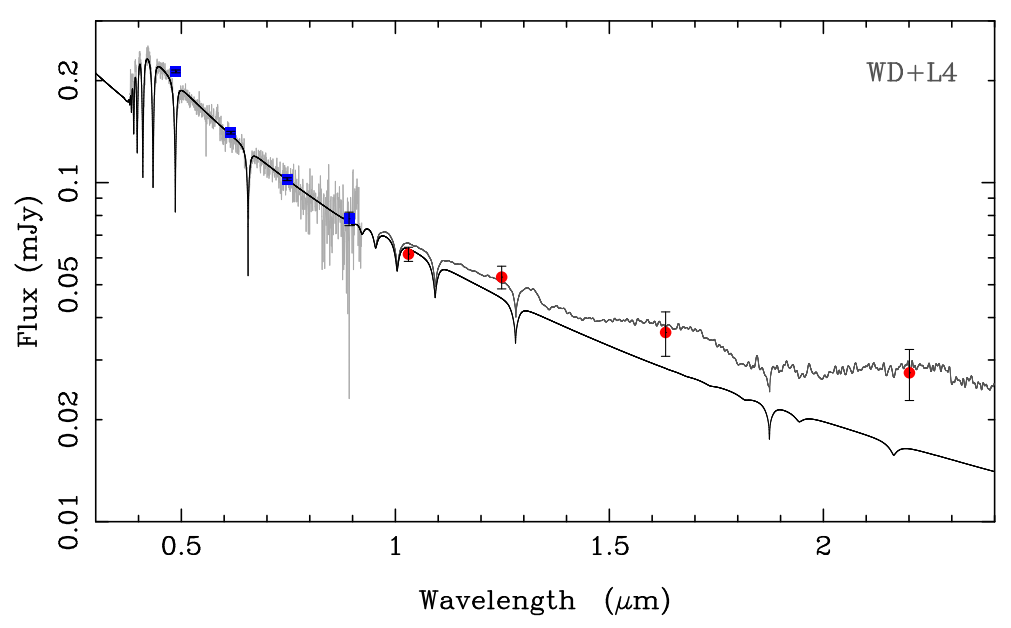

FIGURE A.5. SDSS J090759.59+053649.7 model spectrum (solid black) with SDSS griz' (blue) and UKIDSS $Y J H K$ photometry (red). Also shown are the SDSS spectrum (light grey) and a composite WD+L4 spectrum (dark grey).

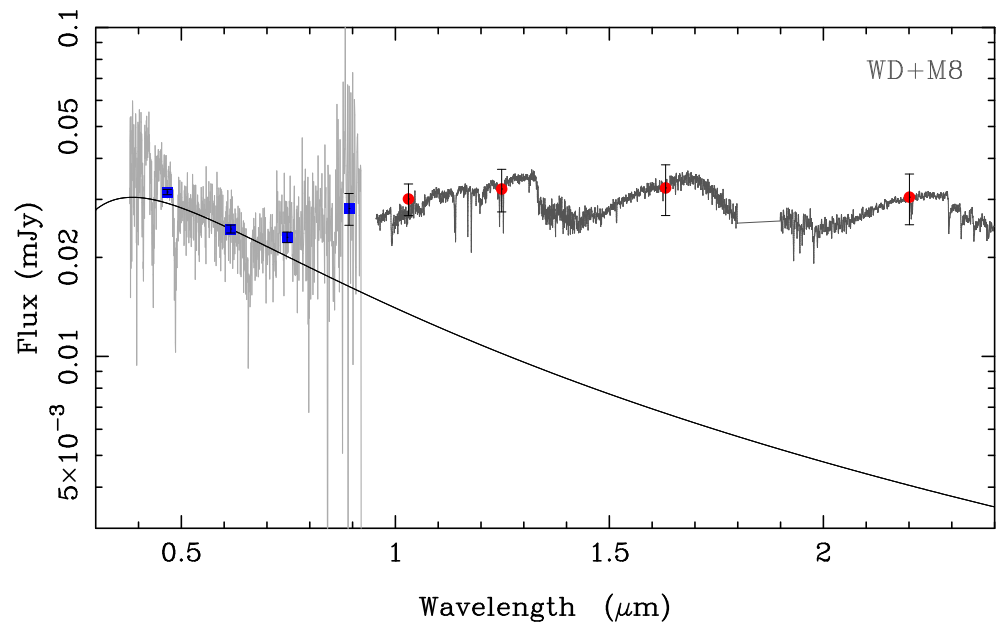

FIGURE A.6. SDSS J092648.48+102828.8 model spectrum (solid black) with SDSS griz' (blue) and UKIDSS YJHK photometry (red). Also shown are the SDSS spectrum (light grey) and a composite WD+M8 spectrum. (dark grey). 


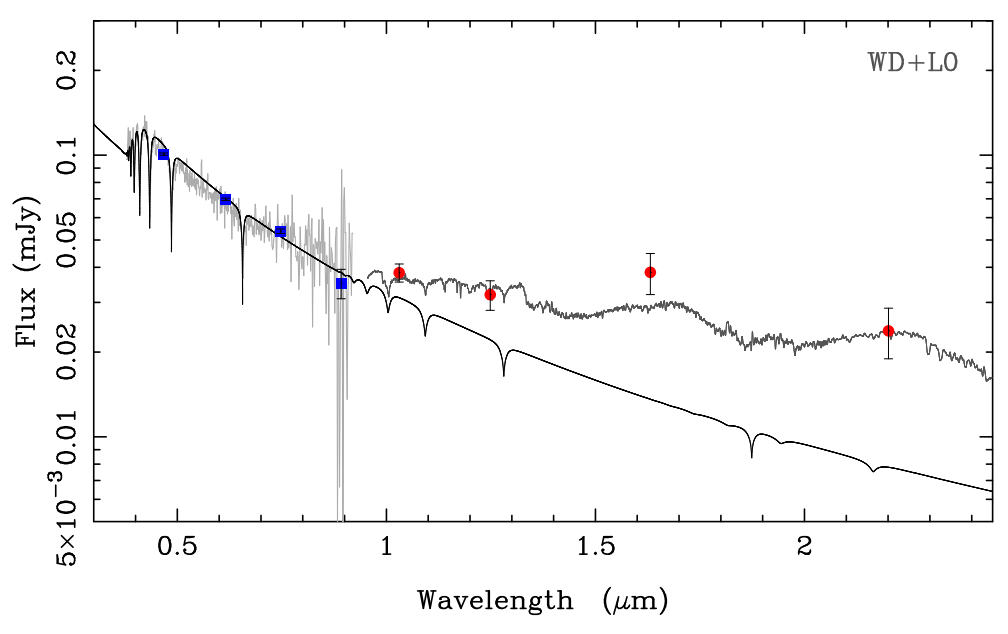

FIGURE A.7. SDSS J100259.88+093950.0 model spectrum (solid black) with SDSS griz $^{\prime}$ (blue) and UKIDSS Y $J H K$ photometry (red). Also shown are the SDSS spectrum (light grey) and a composite WD+L0 dwarf spectrum (dark grey).

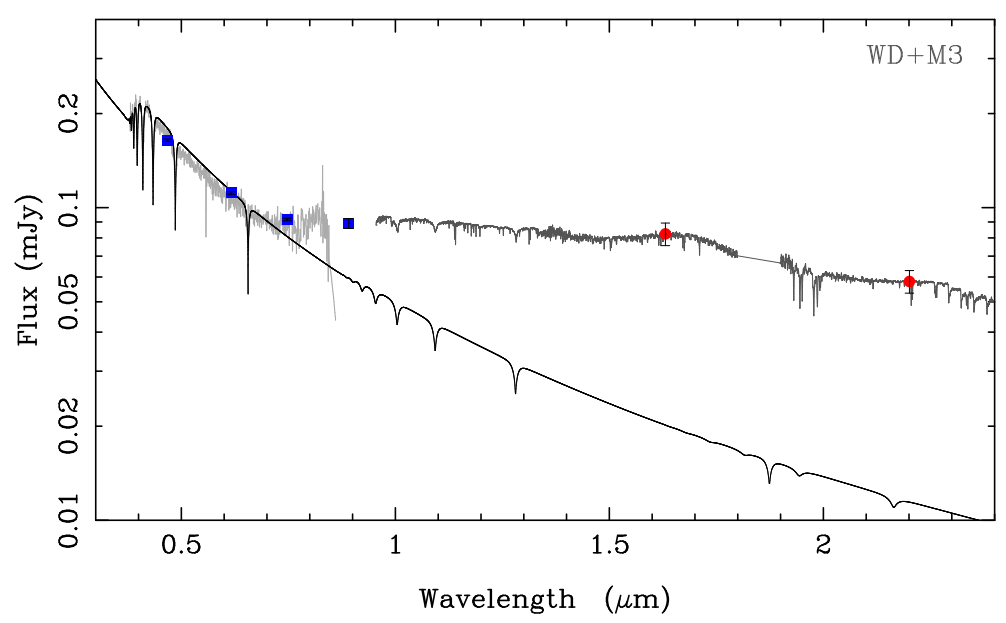

FIGURE A.8. SDSS J101642.93+044317.7 model spectrum (solid black) with SDSS $g r i z^{\prime}$ (blue) and UKIDSS $H K$ photometry (red). Also shown are the SDSS spectrum (light grey) and a composite $\mathrm{WD}+\mathrm{M} 3$ dwarf spectrum (dark grey). 


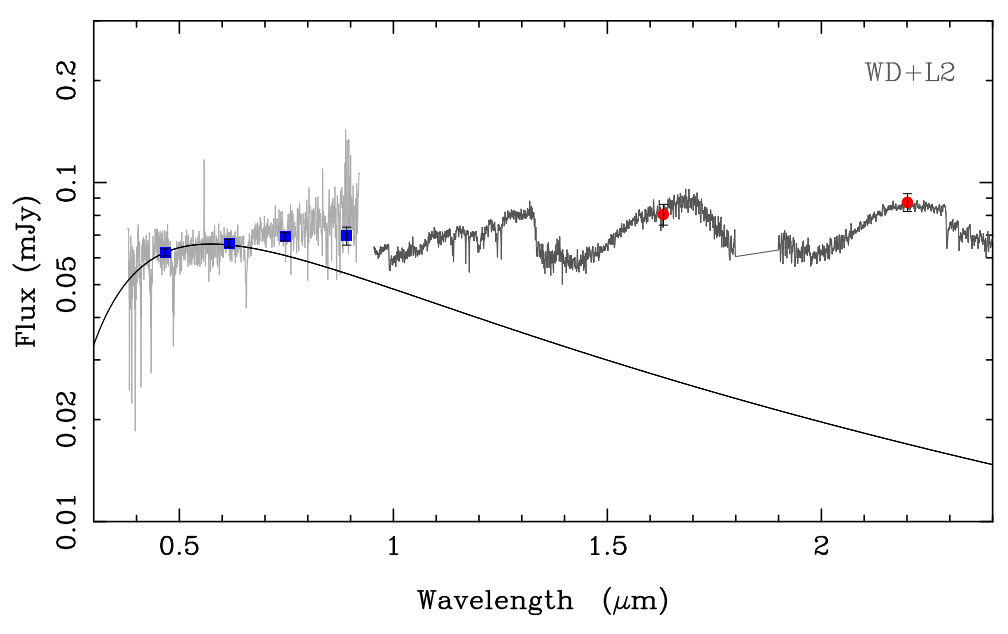

FIGURE A.9. SDSS J103220.19+011227.0 model spectrum (solid black) with SDSS $g r i z^{\prime}$ (blue) and UKIDSS $H K$ photometry (red). Also shown are the SDSS spectrum (light grey) and a composite WD+L2 dwarf spectrum (dark grey).

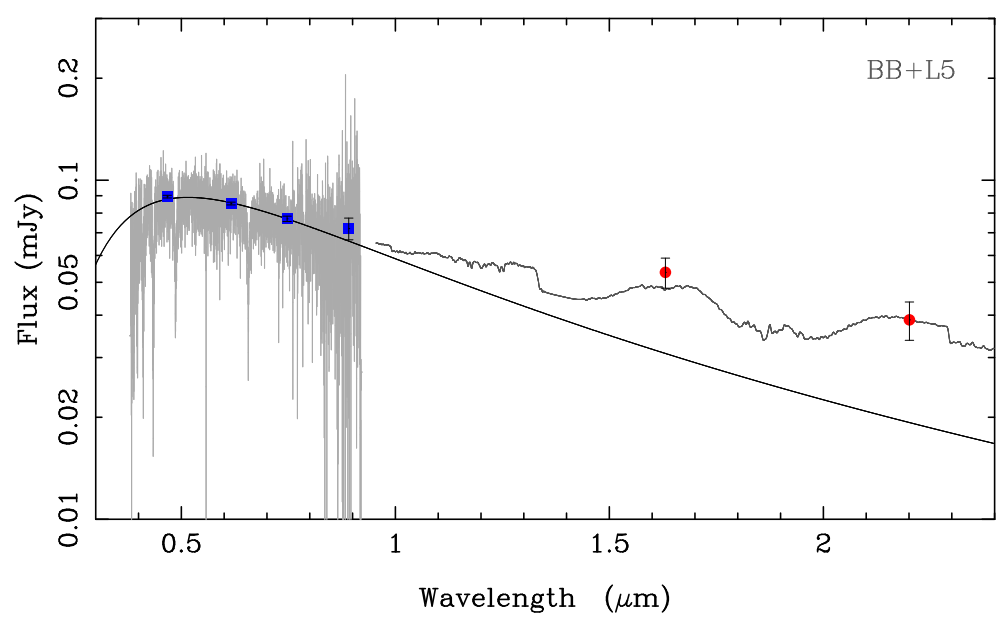

FIGURE A.10. SDSS J103448.92+005201.4 model spectrum (solid black) with SDSS $g r i z^{\prime}$ (blue) and UKIDSS $H K$ photometry (red). Also shown are the SDSS spectrum (light grey) and a composite WD+L5 dwarf spectrum (dark grey). 


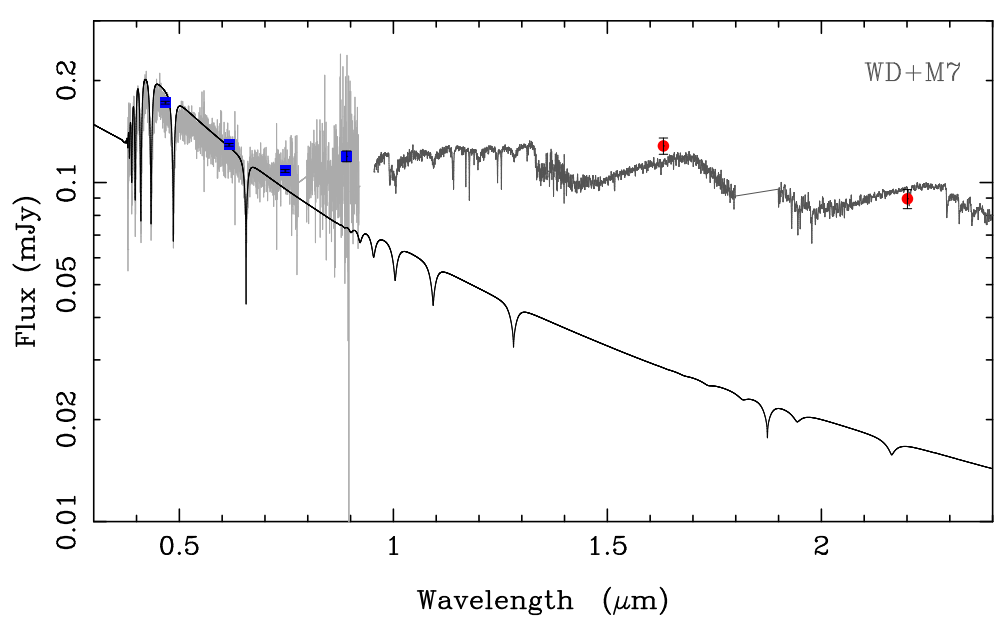

FIGURE A.11. SDSS J103736.75+013912.2 model spectrum (solid black) with SDSS $g r i z^{\prime}$ (blue) and UKIDSS $H K$ photometry (red). Also shown are the SDSS spectrum (light grey) and a composite $\mathrm{WD}+\mathrm{M} 7$ dwarf spectrum (dark grey).

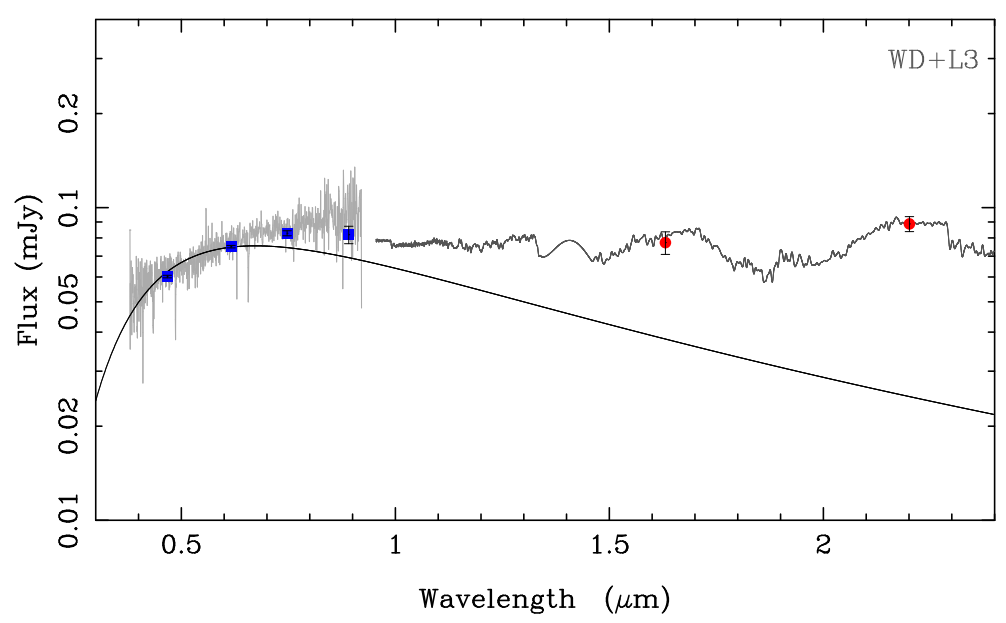

FIGURE A.12. SDSS J104933.58+022451.7 model spectrum (solid black) with SDSS $g r i z^{\prime}$ (blue) and UKIDSS $H K$ photometry (red). Also shown are the SDSS spectrum (light grey) and a composite $\mathrm{WD}+\mathrm{L} 3$ dwarf spectrum (dark grey). 


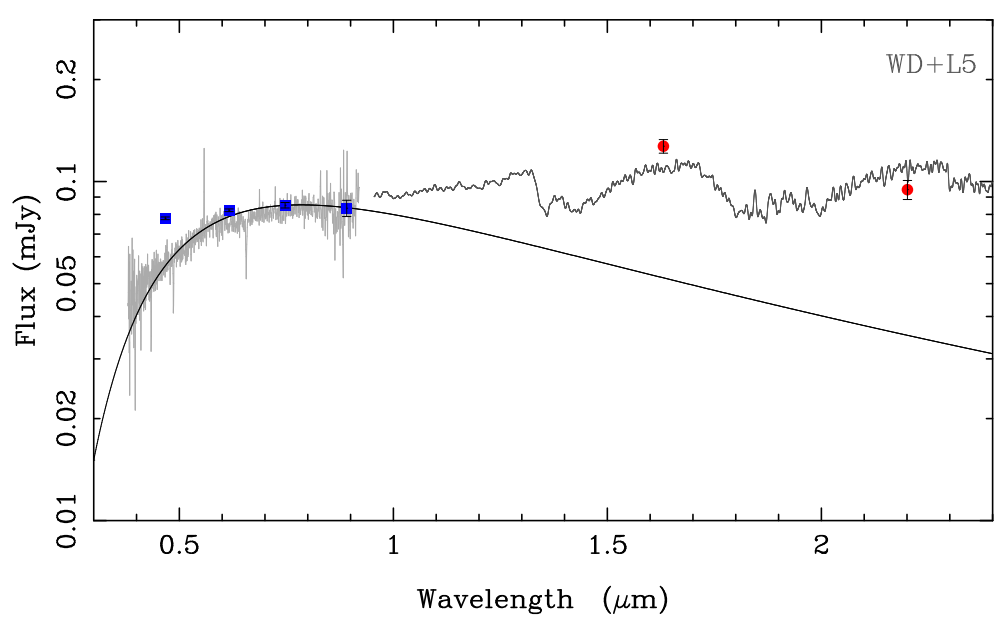

FIGURE A.13. SDSS J105332.62+020126.2 model spectrum (solid black) with SDSS griz' (blue) and UKIDSS $H K$ photometry (red). Also shown are the SDSS spectrum (light grey) and a composite WD+L5 dwarf spectrum (dark grey).

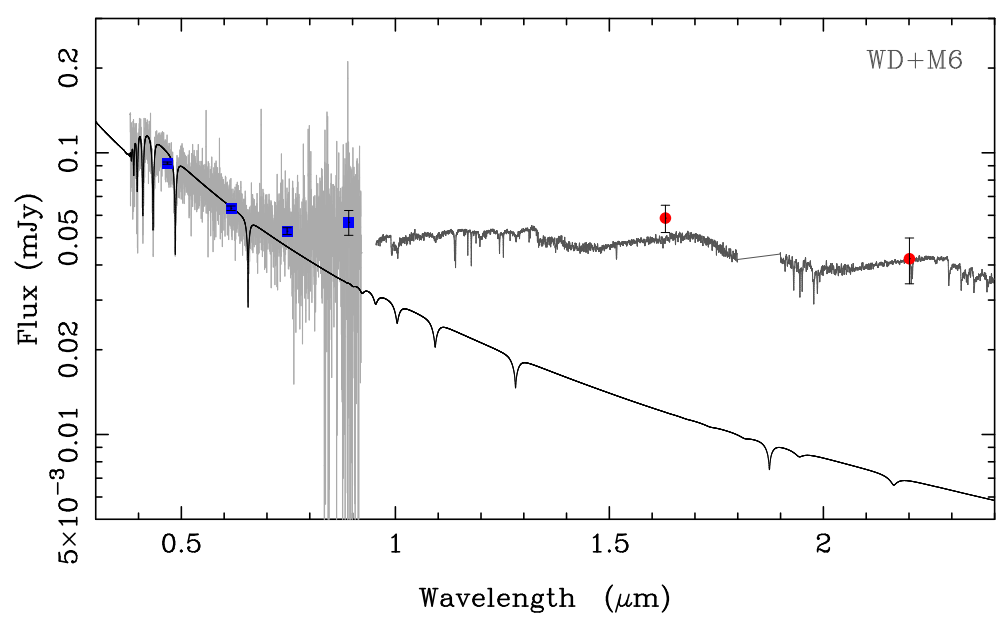

FIGURE A.14. SDSS J113416.09+055227.2 model spectrum (solid black) with SDSS $g r i z^{\prime}$ (blue) and UKIDSS $H K$ photometry (red). Also shown are the SDSS spectrum (light grey) and a composite $\mathrm{WD}+\mathrm{L} 3$ dwarf spectrum (dark grey). 


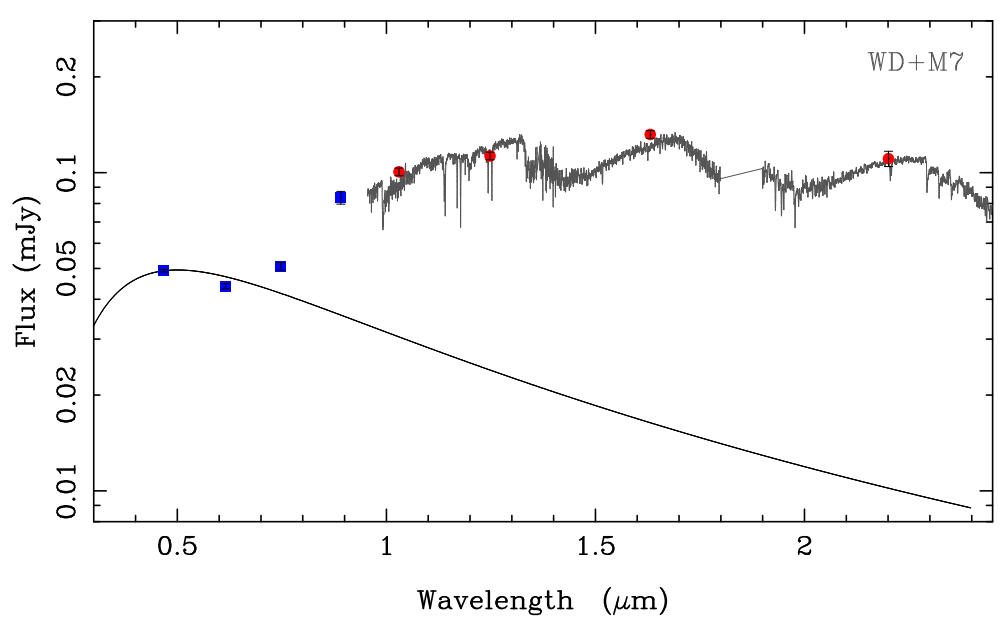

FIGURE A.15. WD1155-011 model spectrum (solid black) with SDSS griz' (blue) and UKIDSS $Y J H K$ photometry (red). Also shown is a composite WD+M7 dwarf spectrum (dark grey).

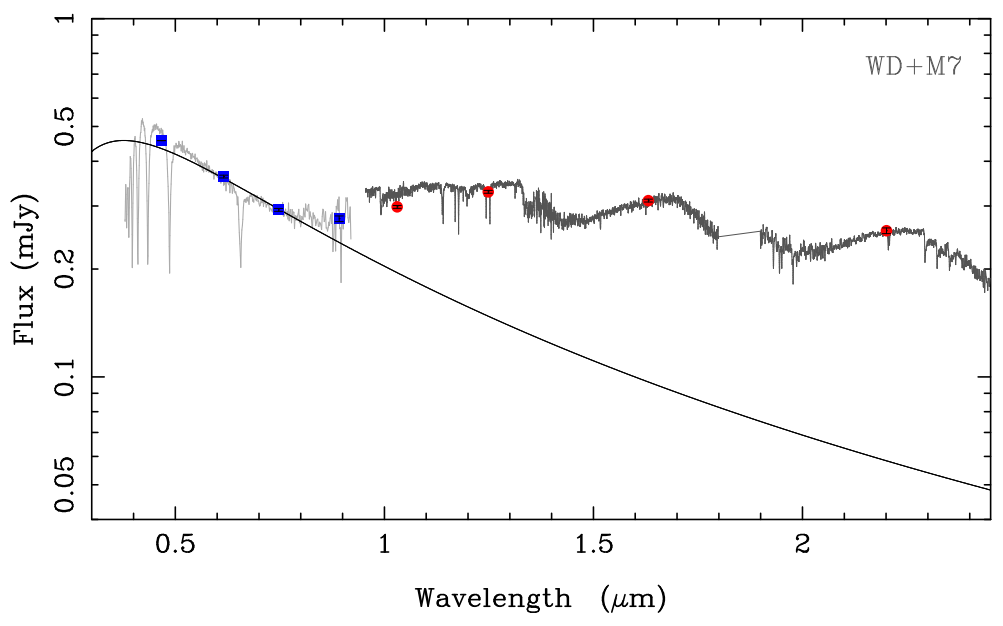

FIGURE A.16. SDSS J132925.21+123025.4 model spectrum (solid black) with SDSS griz' (blue) and UKIDSS $Y J H K$ photometry (red). Also shown are the SDSS spectrum (light grey) and a composite WD+M7 dwarf spectrum (dark grey). 


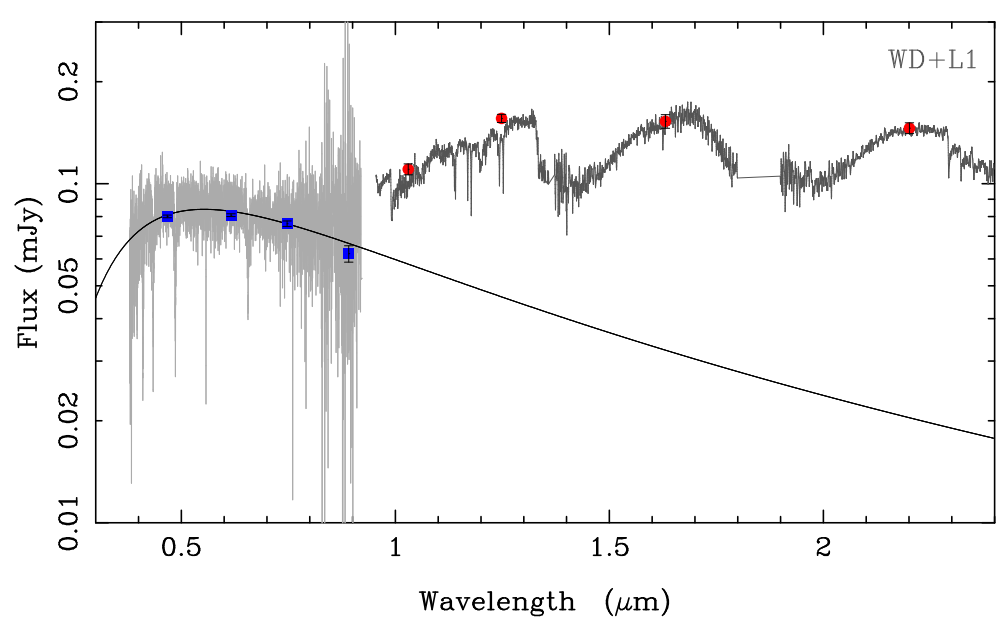

FIGURE A.17. SDSS J220841.63-000514.5 model spectrum (solid black) with SDSS griz' (blue) and UKIDSS $Y J H K$ photometry (red). Also shown are the SDSS spectrum (light grey) and a composite WD $+\mathrm{L} 1$ dwarf spectrum (dark grey).

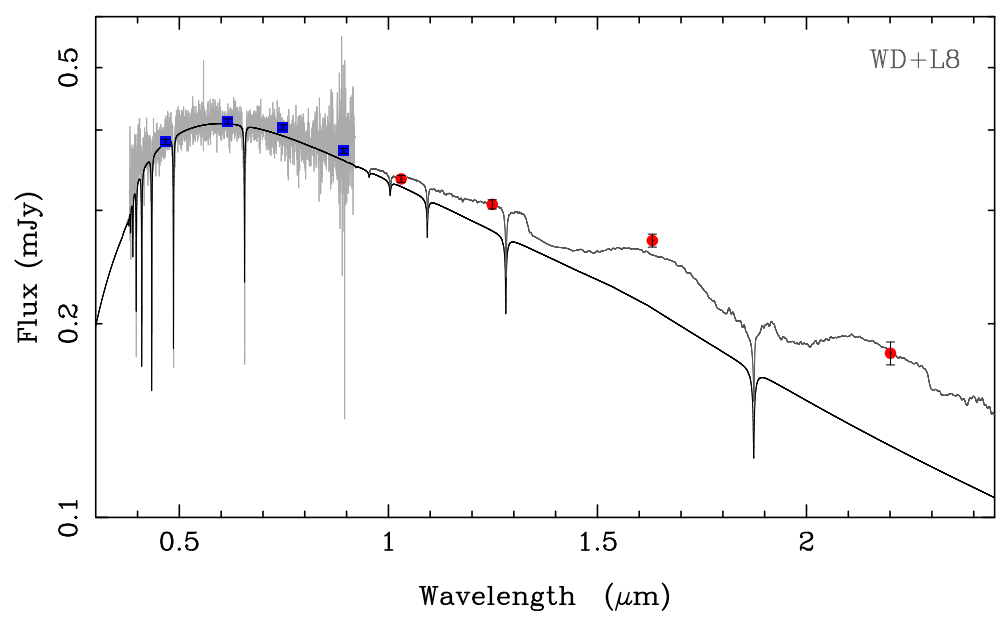

FIGURE A.18. PHL 5038 model spectrum (solid black) with SDSS griz $^{\prime}$ (blue) and UKIDSS $Y J H K$ photometry (red). Also shown are the SDSS spectrum (light grey) and a composite $\mathrm{WD}+\mathrm{L} 8$ dwarf spectrum (dark grey). 


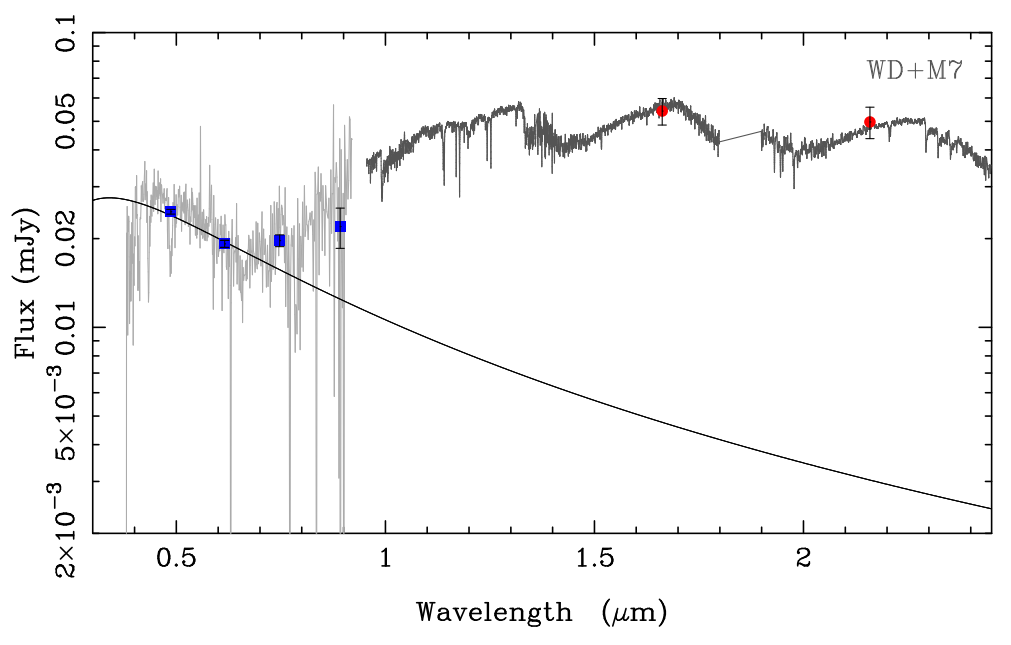

FIGURE A.19. SDSS J233345.97-000843.0 model spectrum (solid black) with SDSS $g_{r i z}$ (blue) and UKIDSS $H K$ photometry (red). Also shown are the SDSS spectrum (light grey) and a composite WD $+\mathrm{M} 7$ dwarf spectrum (dark grey). 


\section{Appendix B}

\section{Chapter 3: Individual Discussions and Spectra}

WD 0419-487 $=V^{*}$ RR Cae

Zuckerman et al. (2003) detected Al I and Si I in the optical spectrum of this white dwarf, which is likely accreted from the wind of a companion. They also detected emission due to $\mathrm{Ca}$ II and thus submitted that this was due to an active or irradiated $\mathrm{M}$ type companion. $V^{*}$ RR Cae is a cool white dwarf which is eclipsed by its M-dwarf companion every 7.3 hours. Maxted et al. (2007) calculated that it will become a CV in 9-20 Gyr and found a spectral type for the companion of M4. Tappert et al. (2007) have analysed this system's $K$-band spectrum and find no evidence for CE accretion. Figure B.1 shows the observed spectrum, modelled with a blackbody (BB) and a composite BB + M4 model. Figures B.2 and B.3 show the model subtracted spectrum with an M4 spectrum for comparison. Starting in the $K$-band the strength of the $\mathrm{Na}$ I at $2.2 \mu \mathrm{m}(\mathrm{EW}=6.5 \pm 0.5 \AA$, Table 3.4) is comparable to the M4 composite as is the strength of the CO bands at $2.3 \mu \mathrm{m}$. The relative strength of the spectral features in the $J$-band are visually consistent with the observed spectrum, again suggesting a mid M-type companion. The overall flux level and strength of the spectral features suggest a companion of spectral type M4, agreeing with Maxted et al. (2007). No evidence in the CO band for CE accretion was found, agreeing with the results of Tappert et al. (2007). 


\section{WD 0752-146 = EGGR 57}

Farihi et al. (2005) used photometry to estimate a spectral type for this companion of M6 orbiting at a distance of $<0.1$ AU. Figure B. 4 shows the observed $H K$ and modelled spectra of EGGR 57 and a composite WD+M6 model. Figures B.5 and B.6 show the model subtracted spectrum with an M6 star for comparison. There is a strong Na I absorption line at $2.2 \mu \mathrm{m}(\mathrm{EW}=8.9 \pm 0.5 \AA$, Table 3.4) suggesting the companion is indeed an M-type star. The visual lack of $\mathrm{Ca} I$ at $2.26 \mu \mathrm{m}$ and the presence of the $\mathrm{CO}$ absorption band at $2.3 \mu \mathrm{m}$ are consistent with a spectral type of M6 confirming the previous estimate. There is no evidence to suggest accretion of material during a $\mathrm{CE}$ phase as the $\mathrm{CO}$ bands appear to have been neither diminished or enhanced by the nuclear processing of CNO.

\section{WD 1305 $+018=$ PG $1305+108$}

PG 1305+108 was confirmed to have a near-infrared excess in its 2MASS photometry by Hoard et al. (2007). They gave a spectral type for the secondary of M1-M3.5 with an angular separation of $<2$ ". This equates to a projected orbital separation of $<96 \mathrm{AU}$ at the distance to the white dwarf (Table 3.4.2). Figure B.7 shows the observed and modelled spectrum of WD 1305+018 and composite WD+M4 and WD+M6 models. Figures B.8 and B.9 show the model subtracted spectrum with an M4 for comparison. The observed flux level indicates an M5 companion as does strengths of the $\mathrm{Na}$ I line at $2.2 \mu \mathrm{m}(\mathrm{EW}=4.7 \pm 0.5 \AA$, Table 3.4$)$ and the $\mathrm{CO}$ absorption at $2.3 \mu \mathrm{m}$, which are visually weak compared to the M4. The same argument applies to a visual comparison of the $\mathrm{Na}$ I line at $1.14 \mu \mathrm{m}$. Therefore it is highly likely that WD $1305+018$ has a companion of spectral type M5. There also appears to be no evidence for the nuclear processing of $\mathrm{CNO}$ in the $\mathrm{CO}$ bands.

\section{WD 1412-049 = PG 1412-049}

PG 1412-049 was identified as having a companion by Wachter et al. (2003) and later resolved with the Hubble Space telescope by Farihi, Hoard \& Wachter (2006b) who, using photometry, assigned a spectral type for the companion as an M0 star at a projected orbital separation of 1200 AU. Figure B.10 shows the observed and modelled spectra of WD 1412-049 and composite WD+M0, WD+M1 and WD+M2 models. Figures B.11 and B.12 show the model subtracted spectrum with an M1 for comparison. However, the strength of absorption features would suggest an earlier spectral type and are more consistent with the previous spectral typing of an M0. At an orbital distance of $1200 \mathrm{AU}$, PG 1412-049 is not a candidate pre-CV and the strength of the CO bands are perfectly 
consistent with the spectral type.

\section{WD 1415 $+132=$ Feige 93}

The observed and modelled spectra of Feige 93 and composite WD+M3, WD+M3.5 and WD+M4 models are shown in Figure B.13. Figures B.14 and B.15 show the model subtracted spectrum with an M3.5 for comparison. An M3.5 companion is consistent with the observed flux level as well as the strength of the spectral features. The CGS4 $J$ band data is particularly noisy with a noticeable rise in flux at approximately $1.3 \mu \mathrm{m}$. A closer inspection of the raw extracted spectrum indicates that this is most likely due to a poor standard correction. Any spectral features in this region would fall within the level of noise. However, based on the UIST $H K$ spectrum where the CO bands can be seen, the companion can be assigned a spectral type of M3.5. The CO bands also do not show evidence of being enhanced or diminished.

WD $1622+323=$ PG $1622+323$

PG $1622+323$ was identified as a binary system by Wachter et al. (2003). Farihi, Hoard $\&$ Wachter (2006b) used photometry to estimate a spectral type for the companion as an M1 orbiting at a distance of 49AU. Figure B.16 shows the modelled and observed spectra of WD $1662+323$ with composite $\mathrm{WD}+\mathrm{M} 0$ and $\mathrm{WD}+\mathrm{M} 0.5$ models. Figures B.17 and B.18 show the model subtracted spectrum with an M0.5 for comparison. Firstly the flux level could indicate an M0.5 companion, as an over-estimate of the distance would have shifted the spectrum downwards. The lack of any strong spectral features in the $J$ band points towards a young M-type companion, although these could be hidden within the noise, as do the weaker line strengths in the UIST $H K$ spectrum. The visual strength of the $\mathrm{CO}$ bands at $2.3 \mu \mathrm{m}$ suggest that the companion could easily lie in the region between $\mathrm{M} 0$ and M1. The weak Na I absorption line at $2.2 \mu \mathrm{m}$ would bias one towards the earlier classification, therefore a companion of spectral type M0.5 is the best fit. At an orbital distance of $49 \mathrm{AU}, \mathrm{PG} 1622+323$ is not a candidate pre-CV.

WD $1643+143=$ PG 1643 +143

PG 1643+143 was first identified by Wachter et al. (2003) as a binary and later Farihi et al. (2005) used photometry to estimate the spectral type of the companion as an M2 orbiting at a distance of $<75 \mathrm{AU}$. Figure B.19 shows the modelled and observed $J H K$ spectra and composite $\mathrm{WD}+\mathrm{M} 1$ and $\mathrm{WD}+\mathrm{M} 2$ models. Figures B. 20 and B.21 shows the 
model subtracted spectrum with an M2 for comparison. The flux level is close to that of an M2 spectral type with the slight difference likely due to errors in distance calibrations. The $\mathrm{Na}$ I lines at $1.14 \mu \mathrm{m}$ and $2.2 \mu \mathrm{m}$ are indicative of an early-M type companion and the strengths of these lines are consistent with the combined WD+M2 model. Therefore, the results are in agreement with the previous photometric estimate of an M2. Although noisy the $\mathrm{CO}$ bands seem to match that of the template $\mathrm{M} 2$ spectrum thus showing no evidence for accretion during a CE phase.

\section{WD 1845+019 = Lanning 18}

Figure B.22 shows the observed and modelled spectra of Lanning 18 and composite $\mathrm{WD}+\mathrm{M} 4, \mathrm{WD}+\mathrm{M} 6$ and $\mathrm{WD}+\mathrm{M} 7$ models. Figures B.23 and B.24 show the model subtracted spectrum with an M4 for comparison. The first thing to note is that the flux level of the CGS4 $J$ band spectrum and the 2MASS $H$ band photometry are consistent with a companion of spectral type M4-M6. Also the relative line strengths in the $J$-band when visually compared with the M4 and M6 spectral types suggests the same conclusion. The flux of the CGS4 $K$-band spectrum then rises towards the level of an M4. The messy water feature at $1.9 \mu \mathrm{m}$ suggests that the spectrum of the standard used for the observation may be to blame - as a poor division during flux calibration could artificially increase the flux. In this case one must look to the absorption features for spectral typing. The $\mathrm{CO}$ band at $2.3 \mu \mathrm{m}$ is consistent with the M4, as is the Na I spectral feature at $2.2 \mu \mathrm{m}$ $(\mathrm{EW}=7.0 \pm 0.6 \AA$, Table 3.4). Therefore the spectrum suggests a companion of spectral type M4.

At first glance it appears that the $\mathrm{CO}$ bands may have been diminished when compared to the template M4 spectrum. However, the first absorption line appears quite prominently and is consistent with the M-dwarf's. Also there is a marked decrease in flux after this initial absorption line possibly indicating the presence of the $\mathrm{CO}$ bands but the resolution was such at the longer wavelengths that these were "averaged out". There is also the poor standard calibration to consider for this object which may have resulted in a loss of definition. In any case there appears to be little evidence to support any CO anomalies at this point.

\section{WD 2151-015 = GJ 4236}

GJ 4236 was first identified as a binary system by Wachter et al. (2003) and the companions was later spectrally typed by Farihi et al. (2005), using photometry, as an M8. Farihi, Hoard \& Wachter (2006b) then went on to resolve this system with the Hubble Space Telescope again giving a spectral type of M8 orbiting at a distance of $23 \mathrm{AU}$. If so it would be the latest $M$ type companion spectroscopically identified in this data set. 
Figure B.26 shows the observed spectrum, modelled with a $8500 \mathrm{~K}$ blackbody and a composite BB +M8 model. Figures B.25 and B.27 show the model subtracted spectrum with an M8 for comparison. The first thing to note is the flux level coincides with the M8 spectral type and also the strength of the Na I line at $2.2 \mu \mathrm{m}(\mathrm{EW}=9.6 \pm 0.4 \AA$, Table 3.4) and the $\mathrm{CO}$ band at $2.3 \mu \mathrm{m}$ is consistent with the template $\mathrm{M} 8$ spectrum. The previous spectral typing as an M8 star is, therefore, in agreement with the observations. At a projected orbital distance of 23 AU, GJ 4236 is not a candidate pre-CV. 


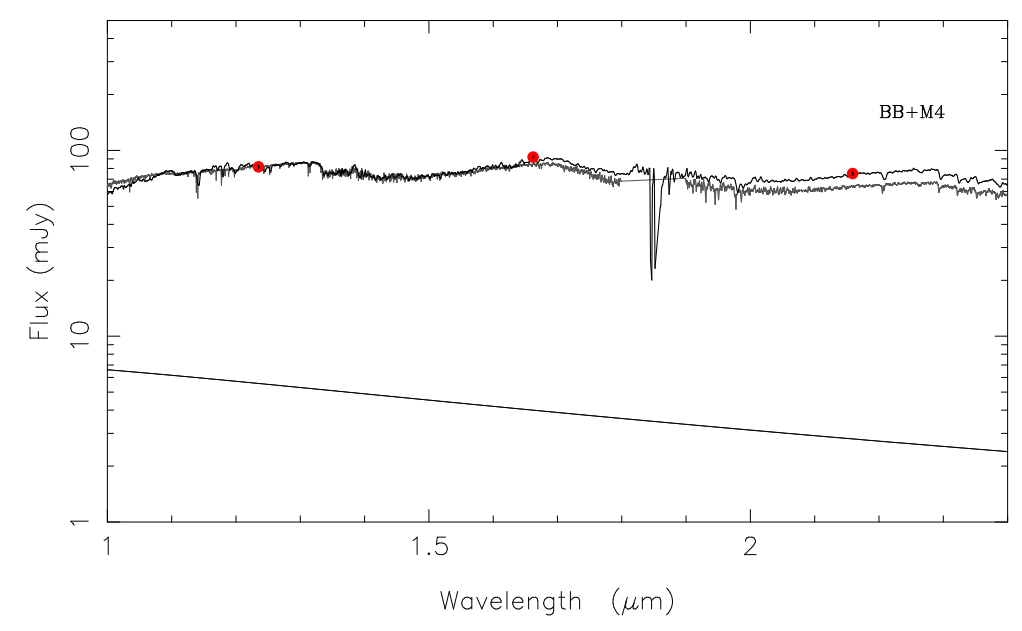

FiguRE B.1. The observed spectrum of V* RR Cae, modelled with a blackbody, with composite models (dashed grey). 2MASS fluxes are also plotted (red). The dashed line indicates a region of very little atmospheric transmission.

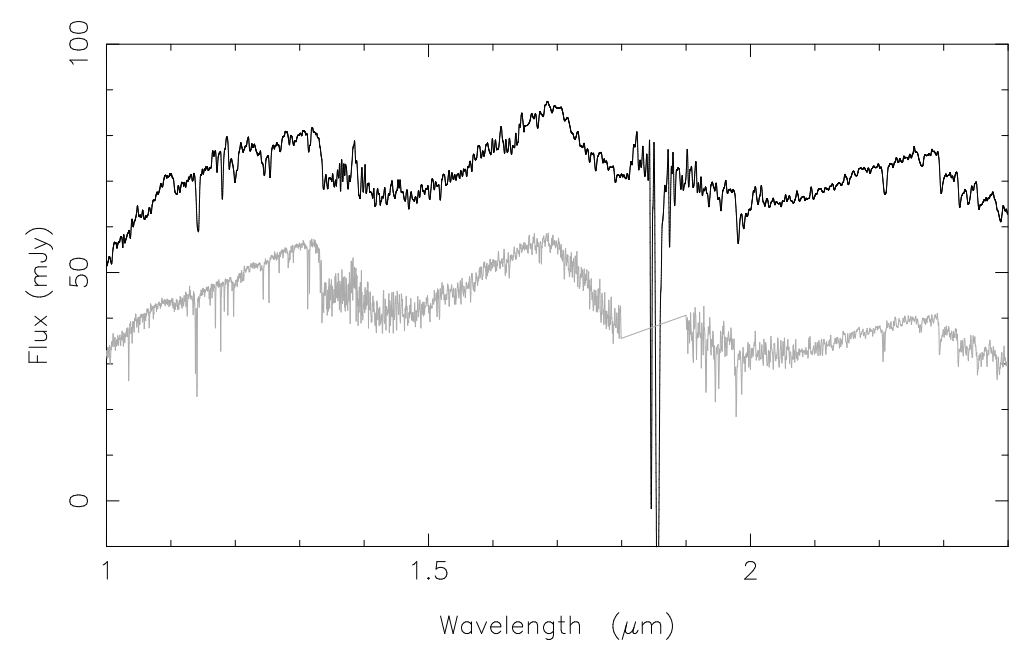

Figure B.2. Model subtracted spectrum of V* RR Cae B with an M4 star for comparison. The dashed line indicates a region of very little atmospheric transmission.

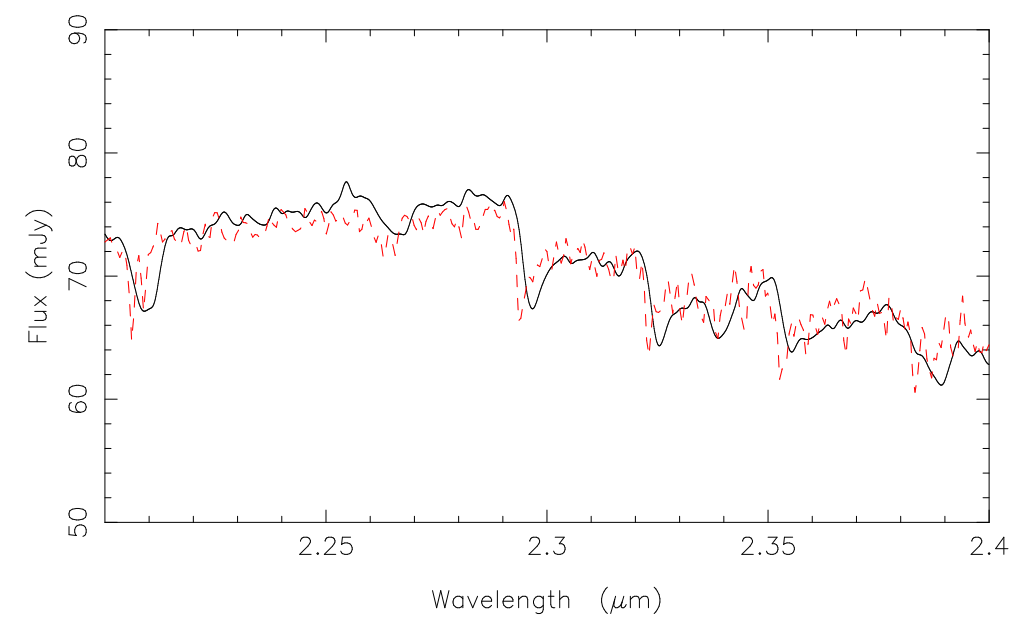

FIgURE B.3. Model subtracted spectrum of $\mathrm{V}^{*} \mathrm{RR}$ Cae showing both the $\mathrm{Na}$ I and $\mathrm{CO}$ absorption lines. An M4 star is overplotted for comparison (red) 


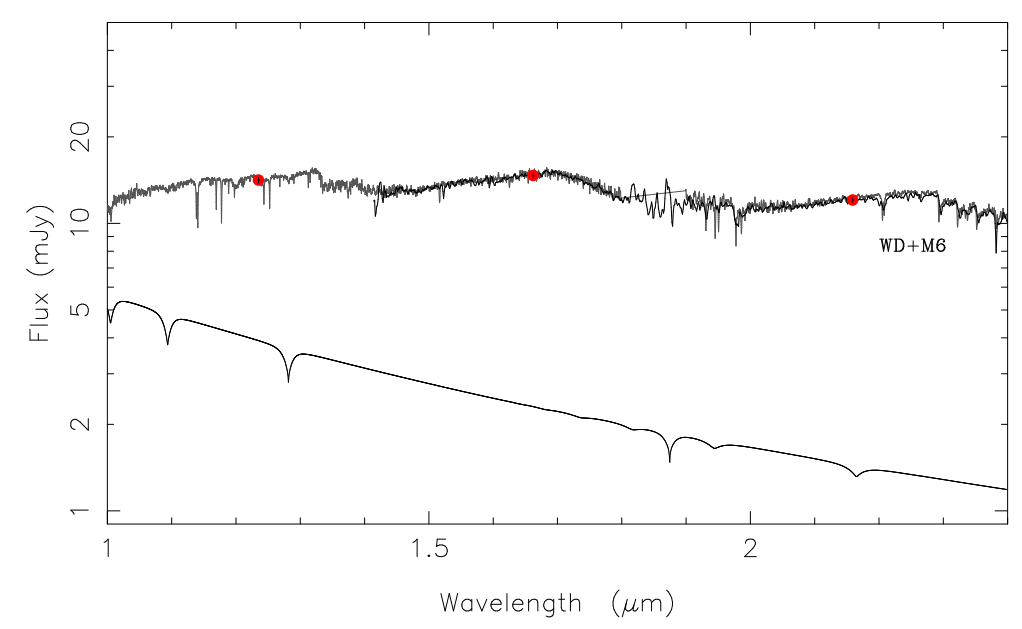

FIGURE B.4. Model and observed spectrum of EGGR 57 with composite model (dashed grey). 2MASS fluxes are also plotted (red). The dashed line indicates a region of very little atmopsheric transmission.

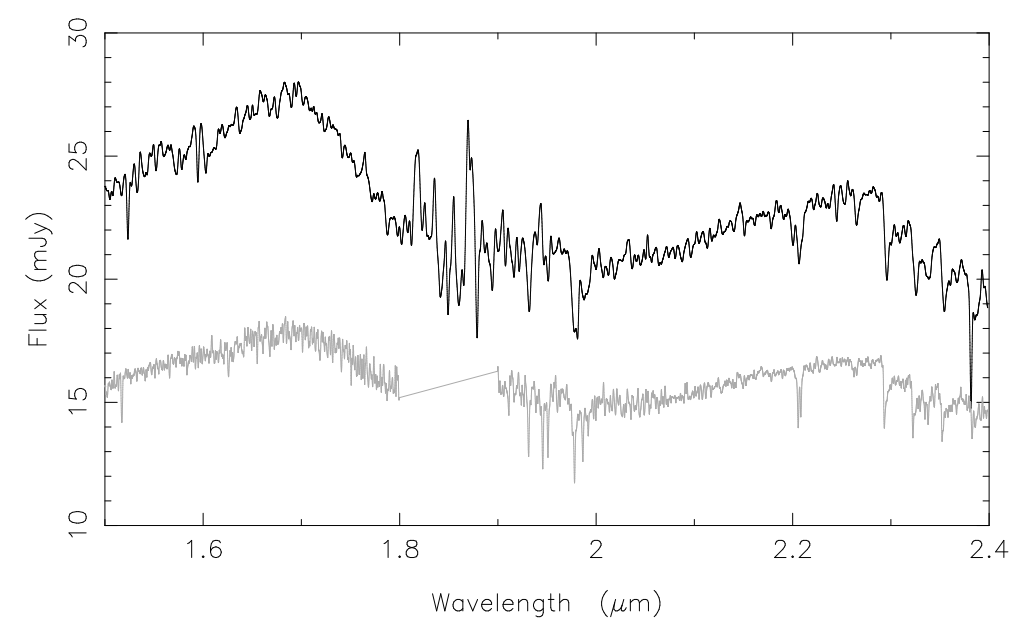

FIGURE B.5. Model subtracted $H K$ spectrum of EGGR 57B with an M6 star (grey) for comparison. The dashed line indicates a region of very little atmospheric transmission.

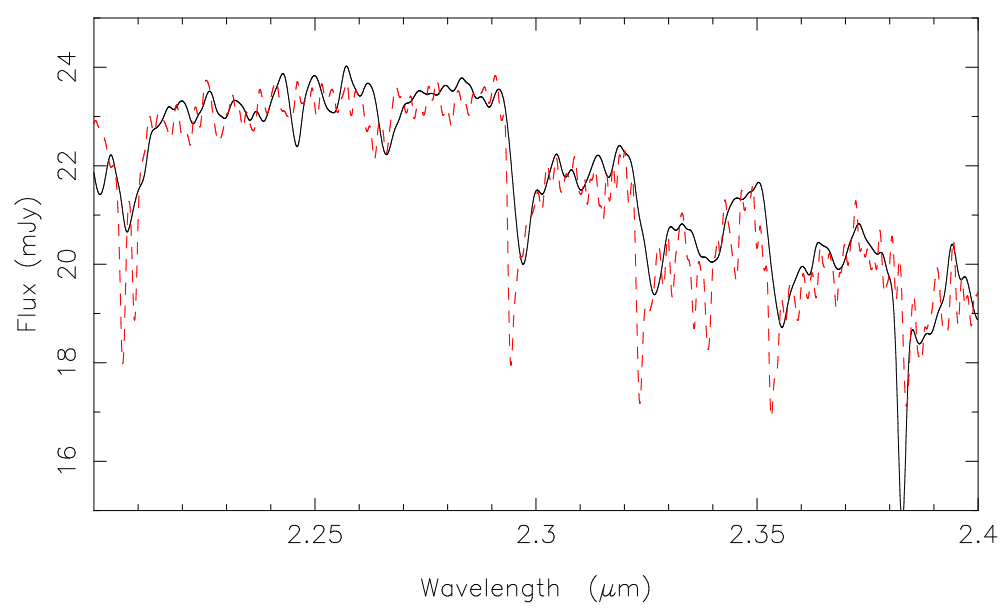

FIGURE B.6. Model subtracted spectrum of EGGR 57 showing both the Na I and $\mathrm{CO}$ absorption lines. An M6 star is overplotted for comparison (red) 


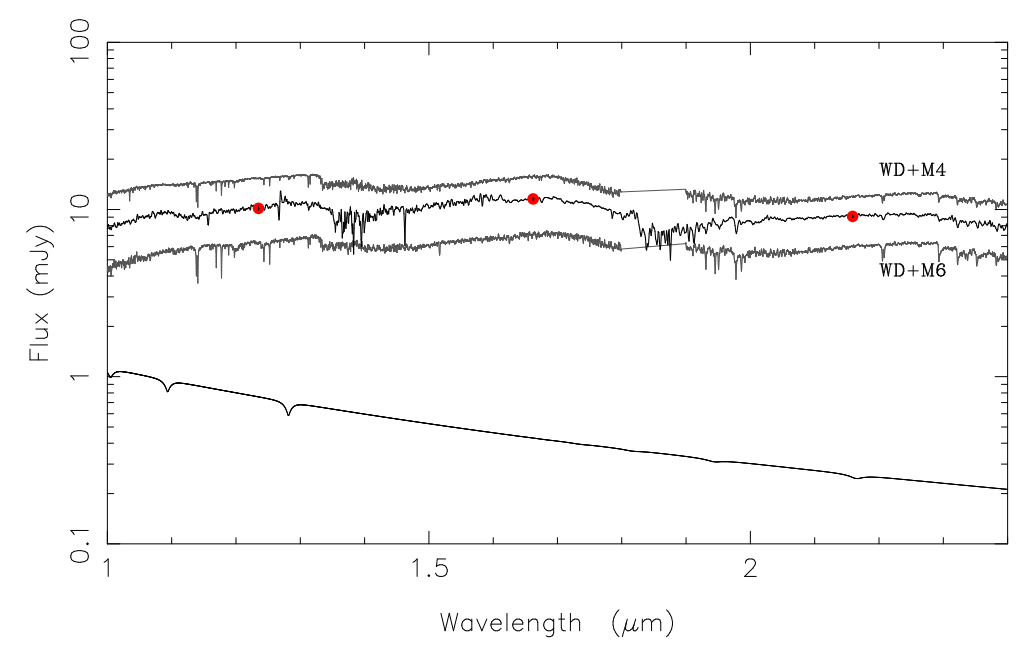

FIGURE B.7. Model and observed spectrum of PG 1305+018 with composite models (dashed grey). 2MASS fluxes are also plotted (red). The dashed line indicates a region of very little atmopsheric transmission.

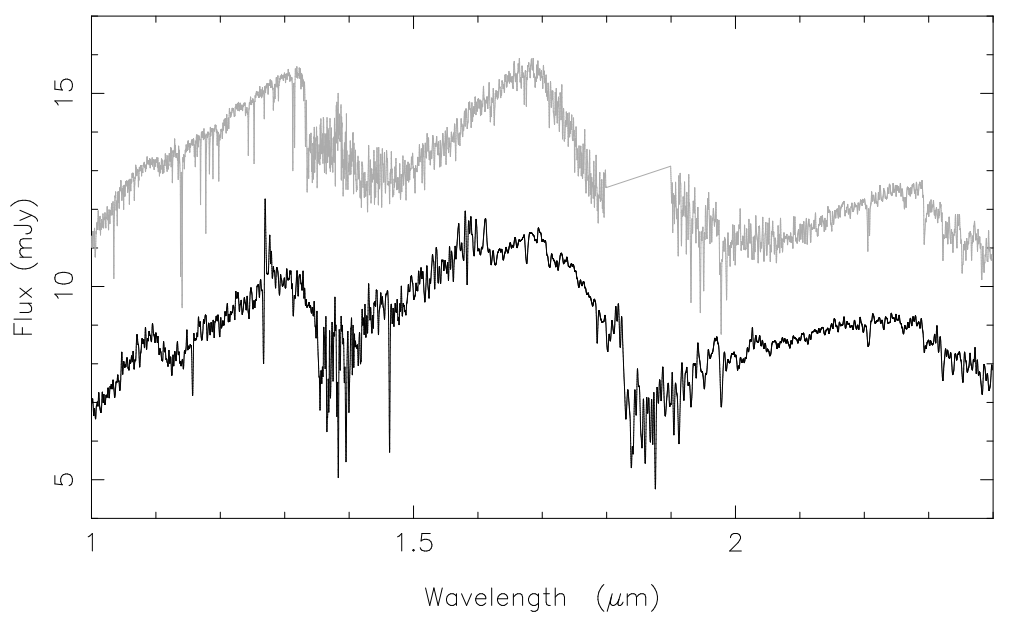

FiguRE B.8. Model subtracted spectrum of PG 1305+018B with an M4 star (grey) for comparison. The dashed line indicates a region of very little atmospheric transmission.

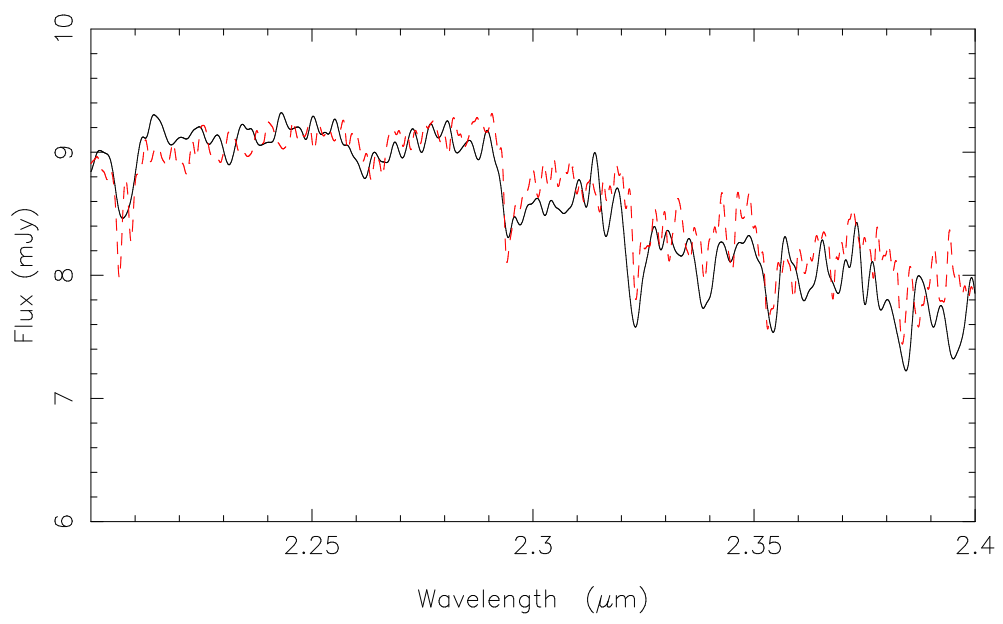

FIGURE B.9. Model subtracted spectrum of PG $1305+018$ showing both the Na I and CO absorption lines. An M4 star is overplotted for comparison (red) 


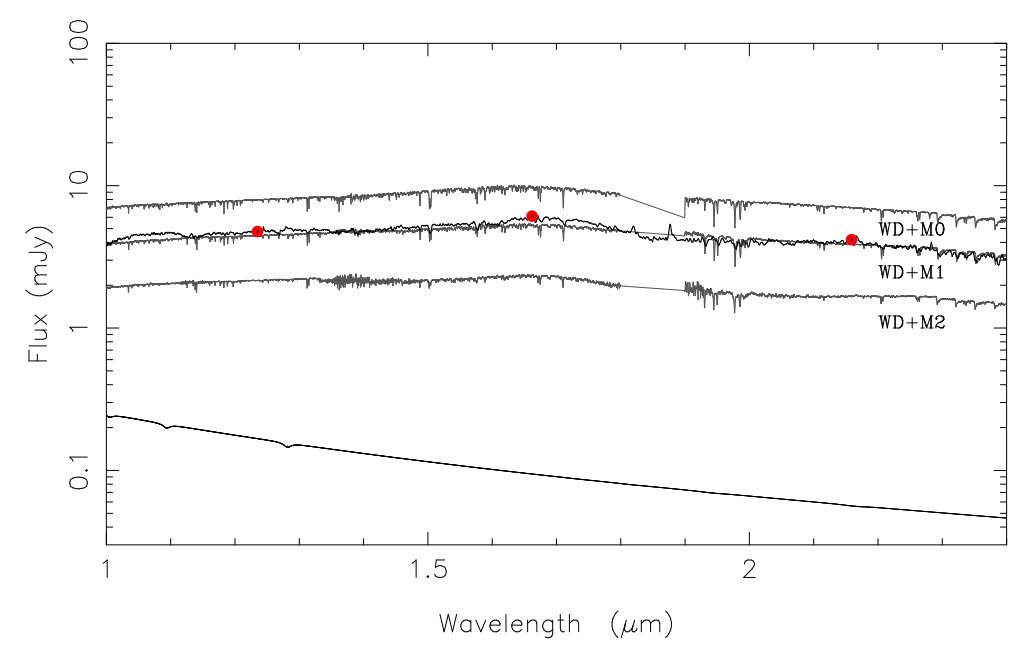

FIGURE B.10. Model and observed spectrum of PG 1412-049 with composite models (dashed grey). 2MASS fluxes are also plotted (red). The dashed line indicates a region of very little atmospheric transmission.

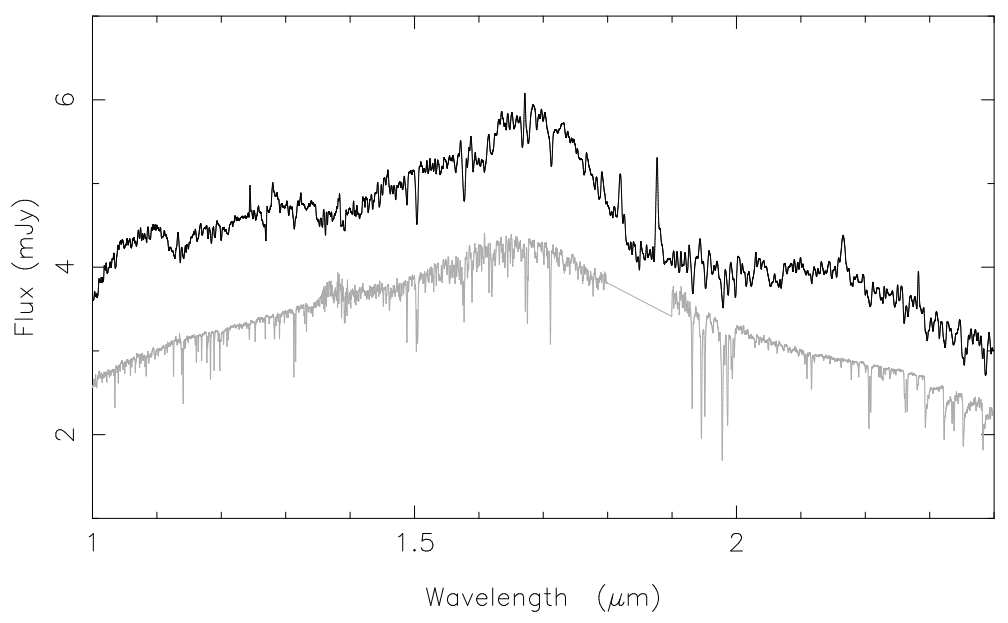

FIGURE B.11. Model subtracted spectrum of PG 1412-049B with an M1 star (grey) for comparison. The dashed line indicates a region of very little atmospheric transmission.

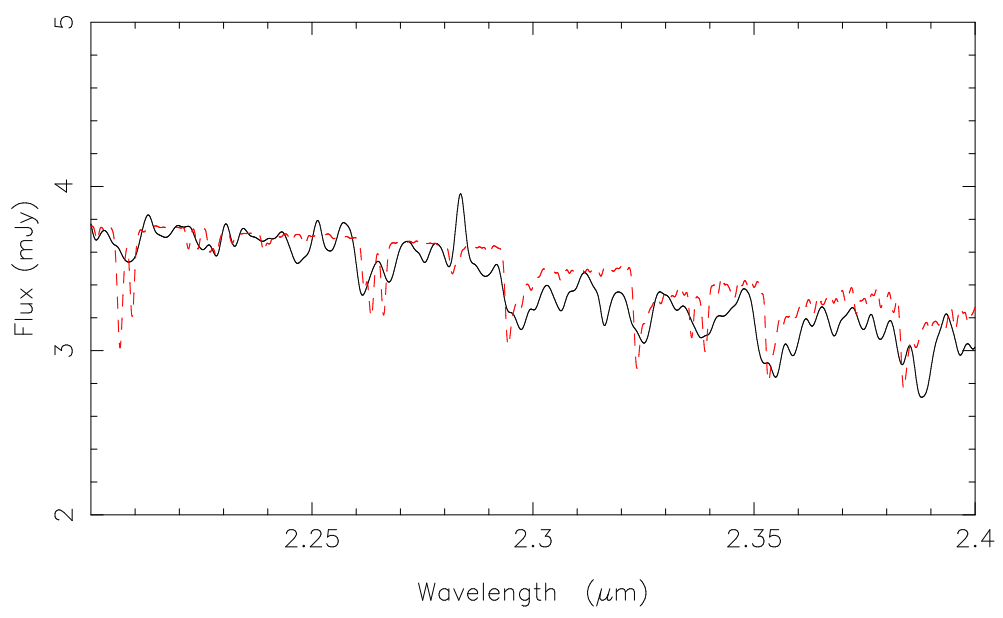

FIgURE B.12. Model subtracted spectrum of PG 1412-049 showing both the Na I and $\mathrm{CO}$ absorption lines. An M1 star is overplotted for comparison (red) 


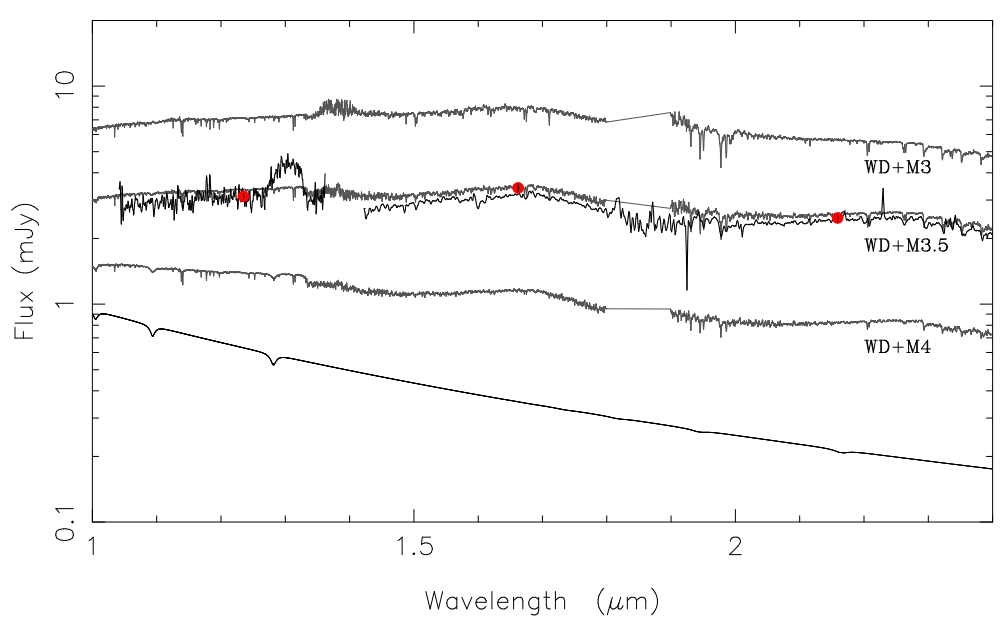

FIGURE B.13. Model and observed spectrum of Feige 93 with composite models (dashed grey). 2MASS fluxes are also plotted (red). The dashed line indicates a region of very little atmospheric transmission.

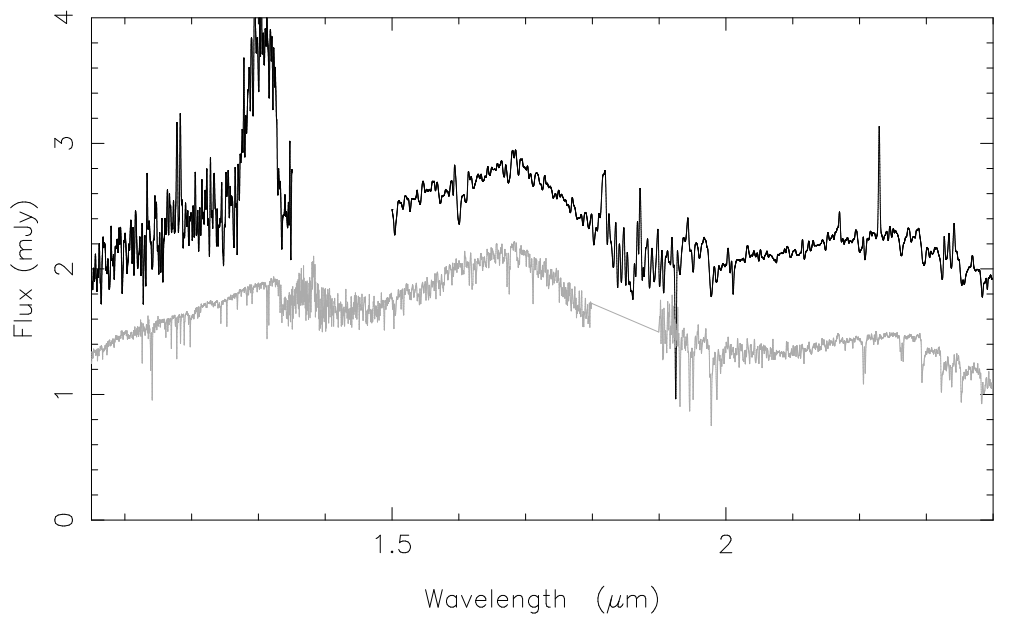

FiguRE B.14. Model subtracted $J$ and $K$ spectra of Feige 93 B with an M3.5 star (grey) for comparison. The dashed line indicates a region of very little atmospheric transmission.

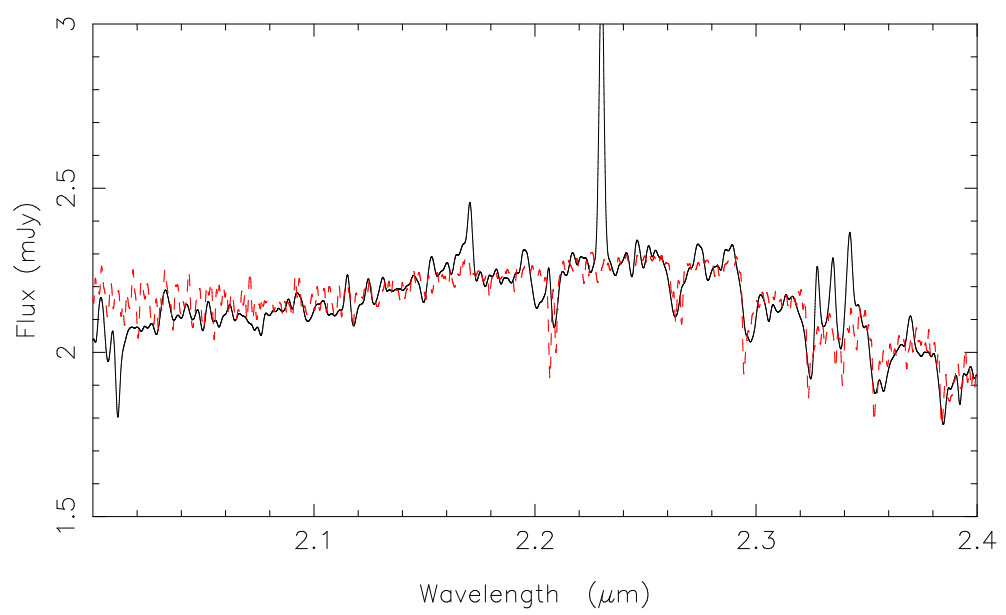

FIGURE B.15. Model subtracted spectrum of Feige 93 showing both the Na I and CO absorption lines. An M3.5 star is overplotted for comparison (red) 


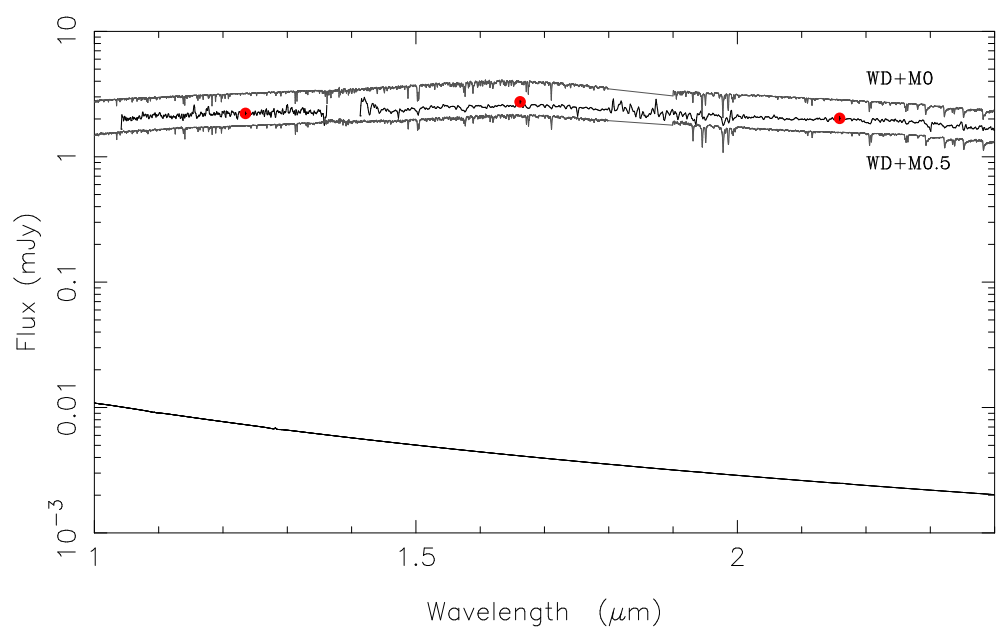

FIGURE B.16. Model and observed spectrum of PG 1622+323 with composite models (dashed grey). 2MASS fluxes are also plotted (red). The dashed line indicates a region of very little atmospheric transmission.

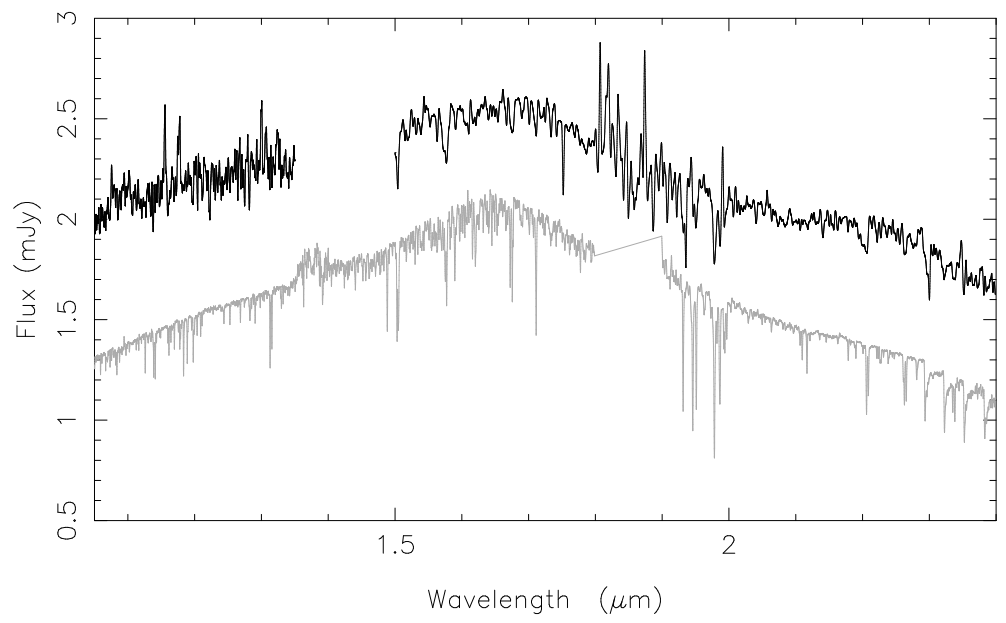

FIGURE B.17. Model subtracted $J$ and $K$ spectra of PG $1622+323$ B with an M0.5 star (grey) for comparison. The dashed line indicates a region of very little atmospheric transmission.

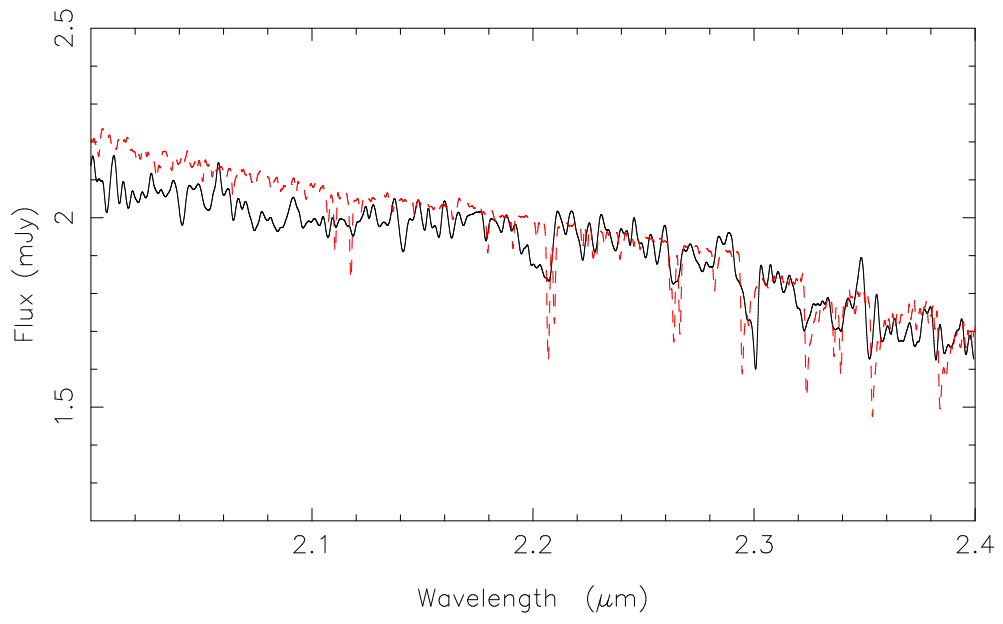

FIGURE B.18. Model subtracted spectrum of PG 1622+323 showing both the Na I and CO absorption lines. An M0.5 star is overplotted for comparison (red) 


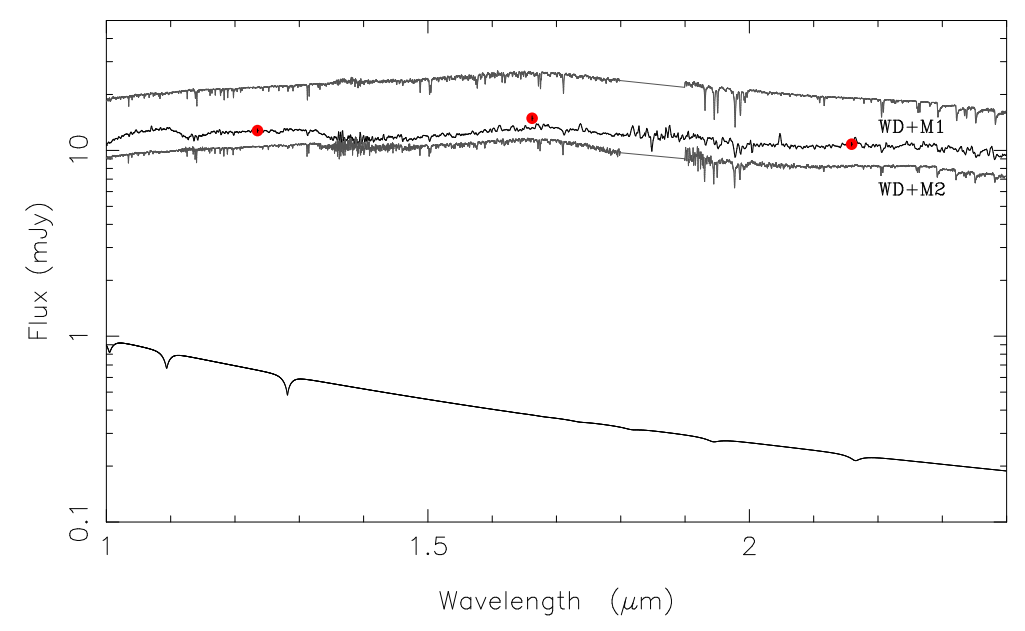

FIGURE B.19. Model and observed spectrum of PG 1643+143 with composite models (dashed grey). 2MASS fluxes are also plotted (red). The dashed line indicates a region of very little atmospheric transmission.

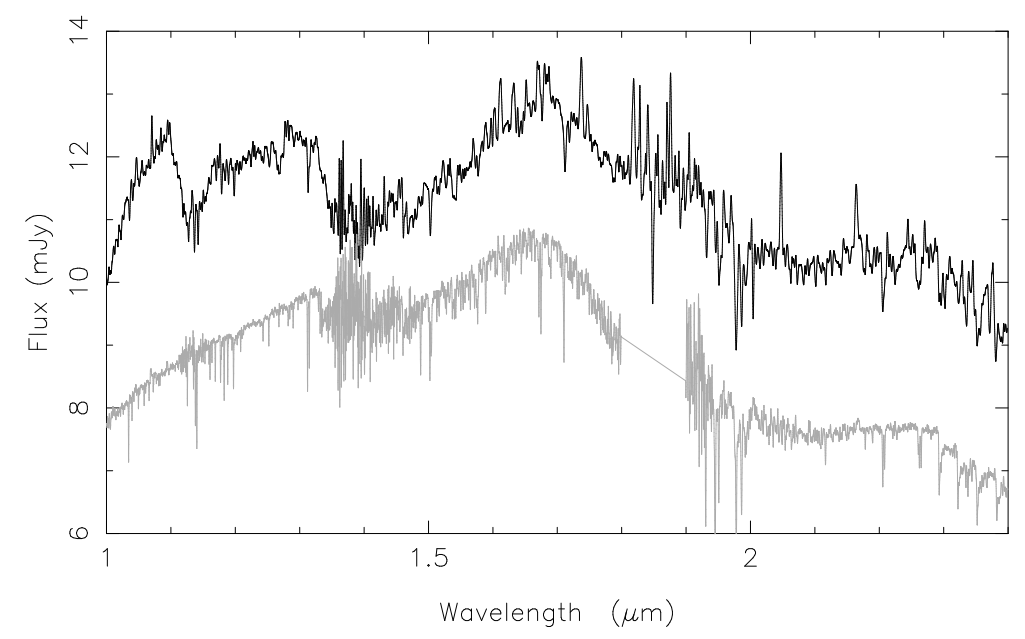

FIGURE B.20. Model subtracted spectrum of PG 1643+143B with an M2 star (grey) for comparison. The dashed line indicates a region of very little atmospheric transmission.

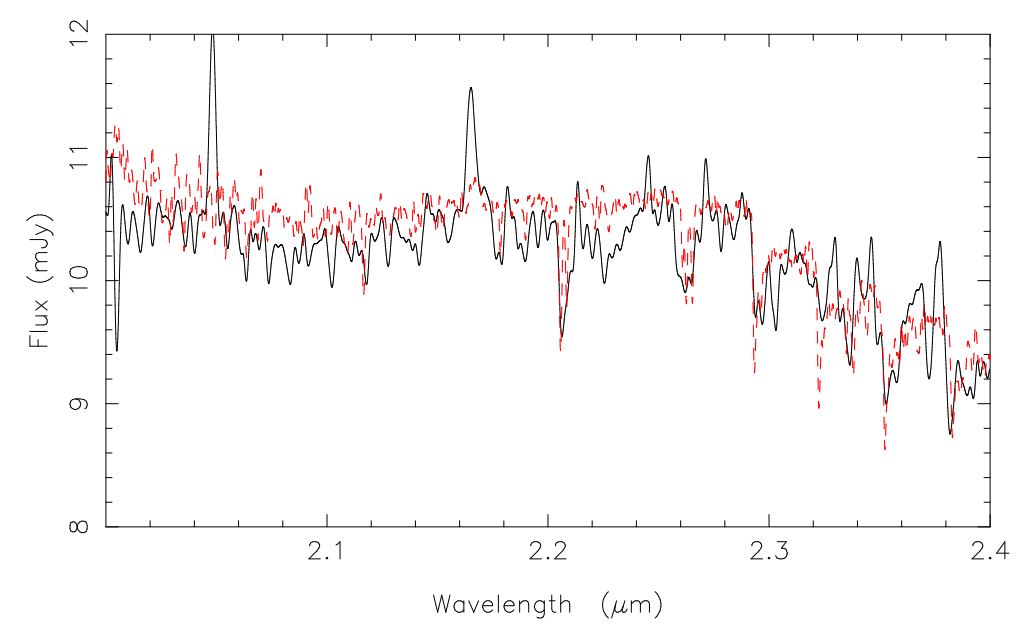

FIGURE B.21. Model subtracted spectrum of PG $1643+143$ showing both the Na I and CO absorption lines. An M2 star is overplotted for comparison (red) 


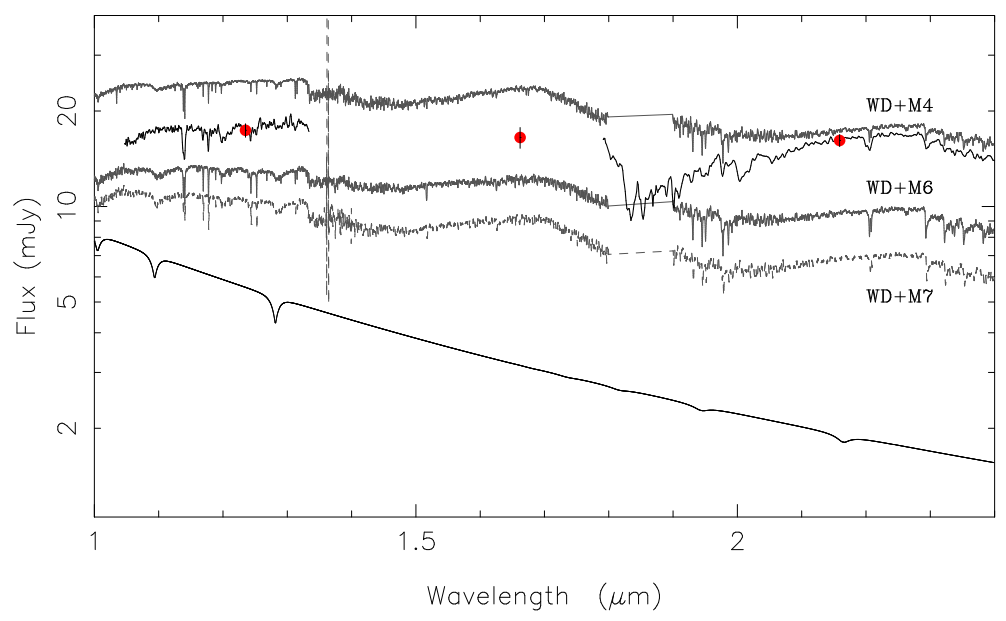

FIGURE B.22. Model and observed spectrum of Lanning 18 with composite models (dashed grey). 2MASS fluxes are also plotted (red). The dashed line indicates a region of very little atmospheric transmission.

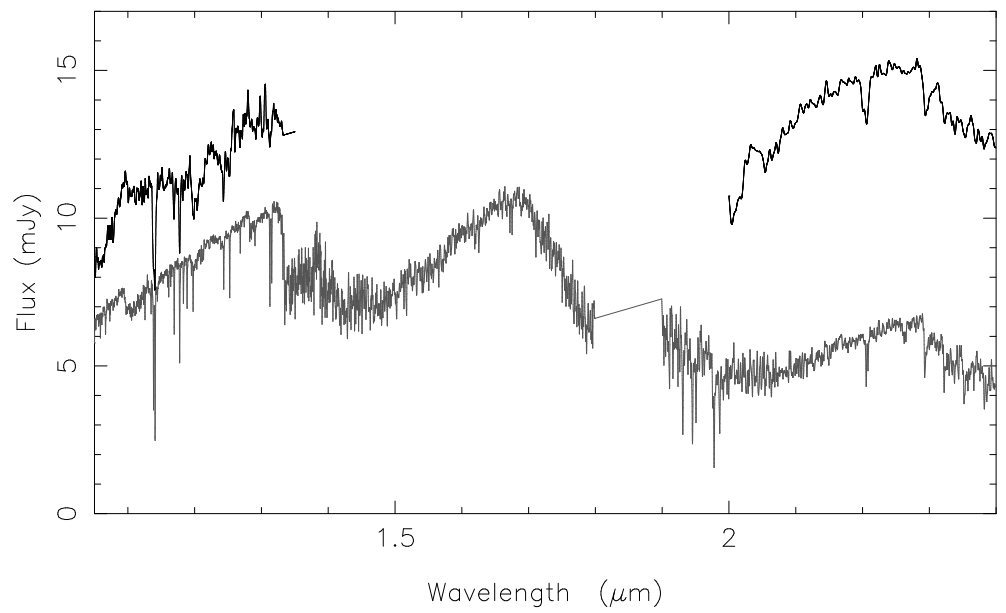

FIGURE B.23. Model subtracted $J$ and $K$ spectra of Lanning 18 B with an M4 star (grey) for comparison. The dashed line indicates a region of very little atmospheric transmission.

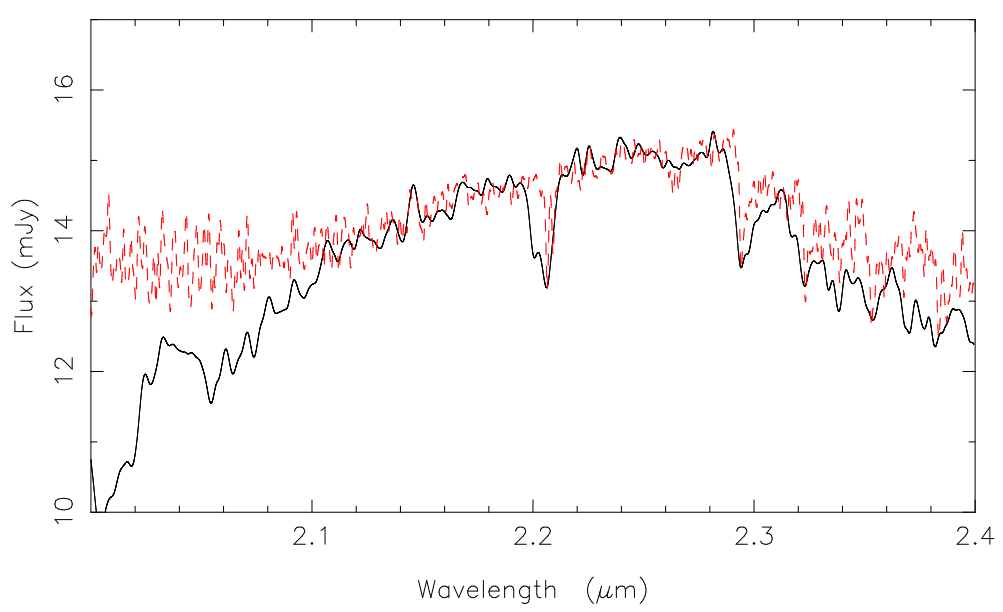

FIGURE B.24. Model subtracted spectrum of Lanning 18 showing both the $\mathrm{Na}$ I and CO absorption lines. An M4 star is overplotted for comparison (red) 


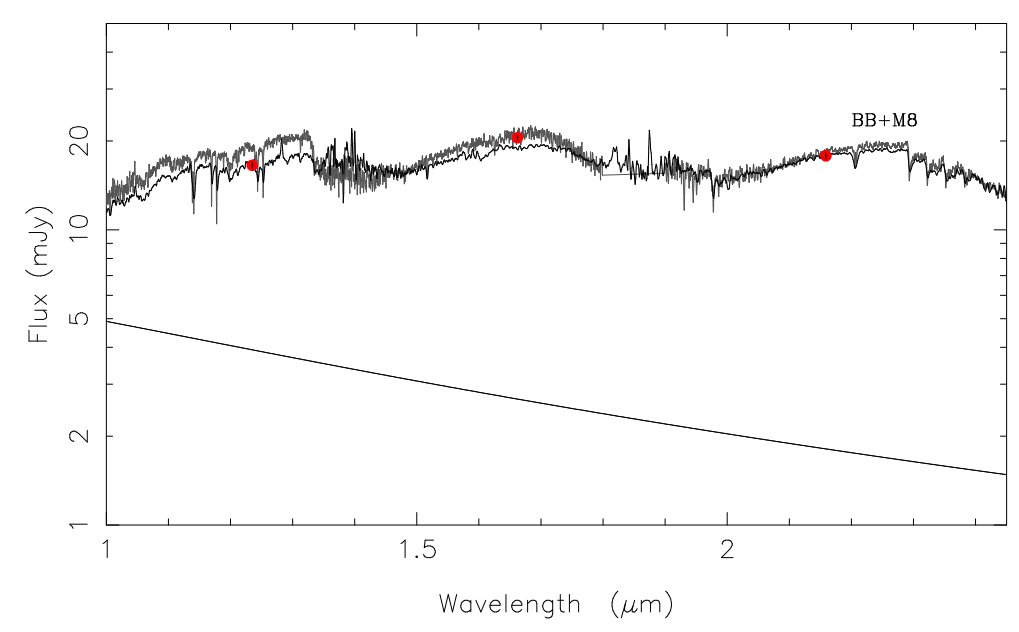

FIGURE B.25. The observed spectrum of GJ 4236, modelled with a blackbody, with composite models (dashed grey). 2MASS fluxes are also plotted (red). The dashed line indicates a region of very little atmospheric transmission.

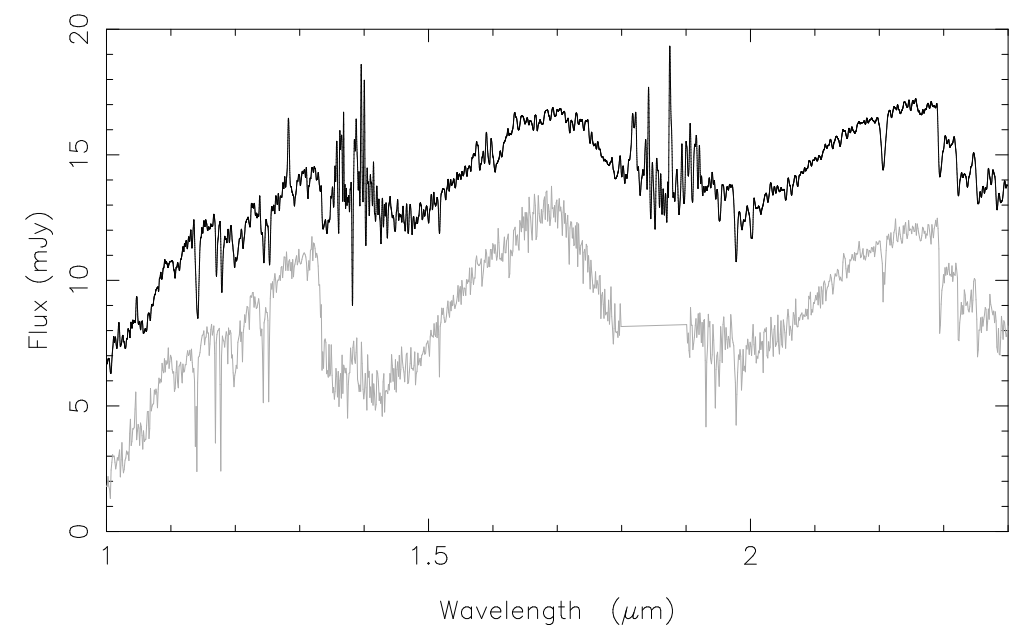

FIGURE B.26. Model subtracted spectrum of GJ 4236B with an M8 star (grey) for comparison. The dashed line indicates a region of very little atmospheric transmission.

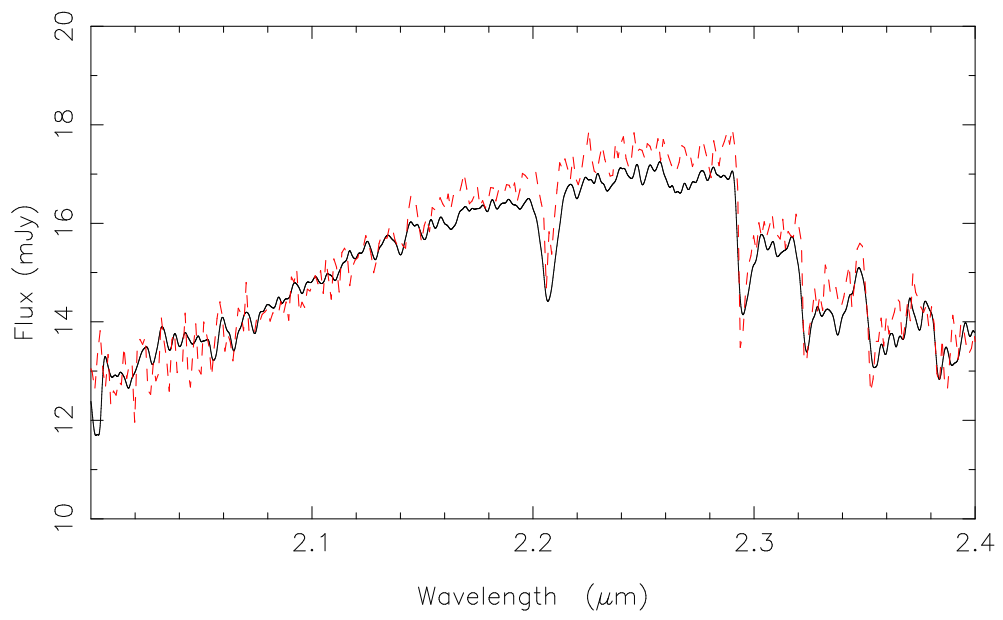

FIGURE B.27. Model subtracted spectrum of GJ 4236 showing both the Na I and CO absorption lines. An M8 star is overplotted for comparison (red) 


\section{Appendix C}

\section{UKIDSS White Dwarf Data}


Table C.1: Physical parameters and UKIDSS data for SDSS DR4 DA

white dwarfs in this work.

\begin{tabular}{|c|c|c|c|c|c|c|c|c|c|c|}
\hline SDSS J & WD & Spec. Type & $i^{\prime}$ & $T_{\text {eff }}(\mathrm{K})$ & $\log g$ & $\mathrm{Y}$ & $\mathrm{J}$ & $\mathrm{H}$ & $\mathrm{K}$ & Ref. \\
\hline $000034.07-010819.9$ & - & DA & 18.240 & $12702 \pm 208$ & $7.98 \pm 0.058$ & $18.03 \pm 0.03$ & $18.15 \pm 0.04$ & $18.12 \pm 0.10$ & $18.37 \pm 0.23$ & 1 \\
\hline $000441.75+152841.1$ & - & DA & 18.587 & $8799 \pm 41$ & $8.3 \pm 0.075$ & - & - & $18.13 \pm 0.12$ & $18.11 \pm 0.19$ & 1 \\
\hline $000636.61+160237.7$ & - & DA & 19.084 & $9564 \pm 71$ & $8.3 \pm 0.098$ & - & - & $18.59 \pm 0.18$ & $18.56 \pm 0.30$ & 1 \\
\hline $000738.03+004003.3$ & - & DA & 18.476 & $10286 \pm 46$ & $8.29 \pm 0.045$ & $18.40 \pm 0.03$ & $18.38 \pm 0.05$ & $18.49 \pm 0.18$ & $18.60 \pm 0.31$ & 1 \\
\hline $000946.45+144310.6$ & - & DA & 17.820 & $24266 \pm 245$ & $7.95 \pm 0.026$ & $17.80 \pm 0.03$ & $17.82 \pm 0.04$ & $18.10 \pm 0.13$ & $17.86 \pm 0.19$ & 1 \\
\hline $001427.04+135058.4$ & - & DA & 18.391 & $8998 \pm 42$ & $8.48 \pm 0.065$ & $18.14 \pm 0.03$ & $17.96 \pm 0.04$ & $17.81 \pm 0.09$ & $18.04 \pm 0.18$ & 1 \\
\hline $001518.88+135332.8$ & - & DA & 17.314 & $8635 \pm 28$ & $8.34 \pm 0.046$ & $16.99 \pm 0.01$ & $16.89 \pm 0.02$ & $16.80 \pm 0.04$ & $16.76 \pm 0.05$ & 1 \\
\hline $001549.02+010937.3$ & - & $\mathrm{DA}+\mathrm{M}$ & 19.851 & $6306 \pm 10$ & $7.57 \pm 0.653$ & $18.46 \pm 0.04$ & $17.86 \pm 0.03$ & $17.45 \pm 0.07$ & $17.13 \pm 0.08$ & 3 \\
\hline $001629.05-004451.0$ & - & DA & 18.326 & $9531 \pm 52$ & $8.31 \pm 0.074$ & $18.11 \pm 0.03$ & $18.02 \pm 0.04$ & $18.09 \pm 0.08$ & $18.02 \pm 0.15$ & 1 \\
\hline $001643.36+152410.8$ & - & DA & 18.395 & $8336 \pm 40$ & $8.49 \pm 0.062$ & - & - & $17.91 \pm 0.10$ & $17.93 \pm 0.18$ & 1 \\
\hline $001655.51-005604.5$ & - & DA & 18.171 & $15498 \pm 205$ & $7.92 \pm 0.044$ & $18.03 \pm 0.04$ & $18.08 \pm 0.05$ & $18.30 \pm 0.11$ & $18.21 \pm 0.19$ & 1 \\
\hline 001749.24-000955.5 & - & $\mathrm{DA}+\mathrm{M}$ & 16.781 & $10086 \pm 16$ & $8.86 \pm 0.125$ & $15.75 \pm 0.01$ & $15.33 \pm 0.01$ & $14.77 \pm 0.01$ & $14.56 \pm 0.01$ & 2 \\
\hline $001836.15+003151.4$ & - & DA & 17.730 & $11696 \pm 76$ & $7.93 \pm 0.045$ & $17.55 \pm 0.02$ & $17.54 \pm 0.02$ & $17.60 \pm 0.08$ & $17.66 \pm 0.13$ & 1 \\
\hline $001921.68+144105.2$ & - & DA & 19.057 & $8400 \pm 77$ & $8.82 \pm 0.142$ & $18.70 \pm 0.05$ & $18.56 \pm 0.09$ & $18.41 \pm 0.18$ & $17.89 \pm 0.18$ & 1 \\
\hline $002040.09+001106.3$ & 0018-000 & DA & 18.368 & $7743 \pm 42$ & $8.94 \pm 0.075$ & $17.97 \pm 0.03$ & $17.81 \pm 0.05$ & $17.56 \pm 0.07$ & $17.74 \pm 0.14$ & 2 \\
\hline $002049.43+004434.7$ & $0018+004$ & DA & 16.776 & $9160 \pm 10$ & $9.0 \pm 0.0030$ & $16.53 \pm 0.01$ & $16.34 \pm 0.01$ & $16.31 \pm 0.03$ & $16.36 \pm 0.04$ & 2 \\
\hline 002309.05-003342.0 & - & DA & 16.852 & $15517 \pm 81$ & $8.01 \pm 0.016$ & $16.73 \pm 0.01$ & $16.72 \pm 0.02$ & $16.73 \pm 0.04$ & $16.90 \pm 0.07$ & 1 \\
\hline $002620.40+144409.4$ & - & $\mathrm{DA}+\mathrm{M}$ & 16.652 & $8933 \pm 27$ & $8.12 \pm 0.042$ & $15.13 \pm 0.00$ & $14.60 \pm 0.00$ & $14.07 \pm 0.00$ & $13.80 \pm 0.01$ & 3 \\
\hline $002645.53-002531.8$ & - & DA & 17.412 & $8375 \pm 29$ & $8.43 \pm 0.051$ & $16.98 \pm 0.02$ & - & $16.76 \pm 0.04$ & $16.66 \pm 0.07$ & 2 \\
\hline $002910.37+141310.3$ & - & $\mathrm{DA}+\mathrm{M}$ & 19.071 & $6301 \pm 4$ & $8.41 \pm 0.426$ & $17.67 \pm 0.02$ & $17.12 \pm 0.02$ & $16.51 \pm 0.02$ & $16.27 \pm 0.04$ & 3 \\
\hline $003257.12+000631.4$ & - & $\mathrm{DA}+\mathrm{M}$ & 20.162 & $9571 \pm 209$ & $8.69 \pm 0.193$ & $18.83 \pm 0.07$ & $18.26 \pm 0.07$ & $17.66 \pm 0.07$ & $17.46 \pm 0.10$ & 1 \\
\hline $003301.52+005716.8$ & - & $\mathrm{DA}+\mathrm{M}$ & 19.323 & $6302 \pm 5$ & $8.46 \pm 0.42$ & $17.85 \pm 0.02$ & - & $16.79 \pm 0.03$ & $16.55 \pm 0.03$ & 1 \\
\hline $003426.92+151801.7$ & $0031+150$ & DA & 16.743 & $7655 \pm 19$ & $9.0 \pm 0.0060$ & $16.23 \pm 0.01$ & $16.08 \pm 0.01$ & $15.86 \pm 0.02$ & $15.83 \pm 0.03$ & 2 \\
\hline $003508.27+135045.3$ & $0032+135$ & DA & 17.105 & $22173 \pm 166$ & $7.34 \pm 0.024$ & $17.02 \pm 0.01$ & $17.11 \pm 0.02$ & $17.18 \pm 0.04$ & $17.33 \pm 0.09$ & 2 \\
\hline $003511.63+001150.3$ & $0032-000$ & DA & 16.611 & $9540 \pm 18$ & $8.27 \pm 0.022$ & $16.31 \pm 0.01$ & $16.28 \pm 0.01$ & $16.22 \pm 0.02$ & $16.24 \pm 0.04$ & 2 \\
\hline $003628.06-003124.9$ & - & $\mathrm{DA}+\mathrm{M}$ & 18.635 & $6332 \pm 36$ & $7.01 \pm 0.209$ & $17.17 \pm 0.01$ & $16.67 \pm 0.01$ & $16.09 \pm 0.02$ & $15.81 \pm 0.02$ & 3 \\
\hline $003719.12+003139.0$ & $0034+002$ & DA & 17.650 & $10957 \pm 48$ & $8.41 \pm 0.03$ & $17.41 \pm 0.02$ & - & $17.49 \pm 0.06$ & $17.40 \pm 0.10$ & 2 \\
\hline $003731.07+010947.1$ & - & $\mathrm{DA}+\mathrm{M}$ & 19.099 & $6301 \pm 3$ & $8.3 \pm 0.484$ & $17.39 \pm 0.02$ & - & $16.33 \pm 0.02$ & $16.05 \pm 0.03$ & 1 \\
\hline $003801.19+152837.7$ & $0035+152$ & DA & 18.185 & $22067 \pm 193$ & $8.04 \pm 0.028$ & - & - & $18.27 \pm 0.14$ & $18.53 \pm 0.26$ & 2 \\
\hline 003853.16-002926.6 & - & DA & 18.630 & $8488 \pm 47$ & $8.26 \pm 0.104$ & $18.26 \pm 0.04$ & $18.15 \pm 0.06$ & $18.01 \pm 0.12$ & $17.95 \pm 0.17$ & 1 \\
\hline
\end{tabular}

Continued on next page 
Table C.1- continued from previous page

\begin{tabular}{|c|c|c|c|c|c|c|c|c|c|c|}
\hline SDSS J & WD & Spec. Type & $i^{\prime}$ & $T_{\text {eff }}(\mathrm{K})$ & $\log g$ & $\bar{Y}$ & $\mathrm{~J}$ & $\mathrm{H}$ & $\bar{K}$ & Ref. \\
\hline $003902.47-003000.3$ & - & $\mathrm{DA}$ & 19.125 & $12314 \pm 268$ & $7.39 \pm 0.08$ & $18.70 \pm 0.06$ & $18.37 \pm 0.08$ & $17.86 \pm 0.11$ & $17.41 \pm 0.10$ & 1 \\
\hline $003923.04+003534.7$ & - & DA & 19.201 & $10080 \pm 81$ & $7.99 \pm 0.108$ & - & - & $17.48 \pm 0.05$ & $17.04 \pm 0.06$ & 1 \\
\hline $004001.42+005908.8$ & - & DA & 19.167 & $7377 \pm 83$ & $8.87 \pm 0.14$ & - & - & $18.34 \pm 0.11$ & $18.34 \pm 0.20$ & 1 \\
\hline $004022.88-002130.0$ & - & DA & 15.124 & $16159 \pm 62$ & $7.88 \pm 0.012$ & - & - & $14.77 \pm 0.01$ & $14.81 \pm 0.01$ & 2 \\
\hline $004103.11+142248.6$ & $0038+141$ & DA & 18.133 & $22642 \pm 244$ & $7.9 \pm 0.032$ & $18.04 \pm 0.03$ & $18.22 \pm 0.05$ & $18.26 \pm 0.10$ & $18.31 \pm 0.21$ & 2 \\
\hline $004138.68+151104.9$ & $0039+149$ & $\mathrm{DA}+\mathrm{M}$ & 19.344 & $7629 \pm 98$ & $8.73 \pm 0.182$ & $18.37 \pm 0.05$ & $17.91 \pm 0.05$ & $17.40 \pm 0.04$ & $17.32 \pm 0.09$ & 2 \\
\hline $004558.02+145449.8$ & $0043+146$ & DA & 18.015 & $7573 \pm 34$ & $9.0 \pm 0.0080$ & $17.52 \pm 0.02$ & $17.41 \pm 0.03$ & $17.17 \pm 0.04$ & $17.20 \pm 0.07$ & 2 \\
\hline $004610.36+133910.3$ & $0043+133$ & DA & 18.285 & $11077 \pm 71$ & $8.31 \pm 0.057$ & $18.21 \pm 0.04$ & $18.18 \pm 0.06$ & $18.11 \pm 0.14$ & $18.05 \pm 0.19$ & 2 \\
\hline $004815.69+002034.3$ & - & $\mathrm{DA}+\mathrm{M}$ & 19.205 & $9844 \pm 90$ & $8.21 \pm 0.142$ & $17.78 \pm 0.02$ & $17.41 \pm 0.03$ & $17.04 \pm 0.03$ & $16.59 \pm 0.05$ & 3 \\
\hline $005208.42-005134.6$ & - & $\mathrm{DA}+\mathrm{M}$ & 16.635 & $6300 \pm 1$ & $7.17 \pm 0.092$ & $15.07 \pm 0.00$ & $14.55 \pm 0.00$ & $13.96 \pm 0.00$ & $13.69 \pm 0.00$ & 2 \\
\hline $005245.11-005337.2$ & - & $\mathrm{DA}+\mathrm{M}$ & 17.972 & $6300 \pm 1$ & $8.61 \pm 0.094$ & - & $15.73 \pm 0.01$ & $15.21 \pm 0.01$ & $14.91 \pm 0.01$ & 3 \\
\hline $005418.54+005746.2$ & - & $\mathrm{DA}+\mathrm{M}$ & 17.476 & $8372 \pm 47$ & $7.88 \pm 0.103$ & - & $15.41 \pm 0.00$ & $14.87 \pm 0.01$ & $14.60 \pm 0.01$ & 3 \\
\hline 005436.04-004941.9 & - & DA & 18.343 & $8027 \pm 40$ & $8.81 \pm 0.117$ & - & $17.78 \pm 0.05$ & $17.66 \pm 0.08$ & $17.59 \pm 0.13$ & 2 \\
\hline $005457.61-002517.1$ & - & $\mathrm{DA}+\mathrm{M}$ & 18.459 & $16417 \pm 139$ & $7.57 \pm 0.03$ & $17.27 \pm 0.02$ & $16.82 \pm 0.02$ & $16.45 \pm 0.03$ & $16.06 \pm 0.03$ & 2 \\
\hline $005519.90+004039.1$ & - & $\mathrm{DA}+\mathrm{M}$ & 18.717 & $6300 \pm 2$ & $7.41 \pm 0.389$ & - & $16.93 \pm 0.02$ & $16.41 \pm 0.03$ & $16.15 \pm 0.03$ & 3 \\
\hline $010045.94+150659.1$ & $0058+148$ & $\mathrm{DA}+\mathrm{M}$ & 18.769 & $11121 \pm 128$ & $8.06 \pm 0.087$ & $17.51 \pm 0.02$ & $17.03 \pm 0.02$ & $16.44 \pm 0.03$ & $16.25 \pm 0.05$ & 2 \\
\hline $010602.97+004717.0$ & $0103+005$ & DA & 18.234 & $8946 \pm 40$ & $8.44 \pm 0.061$ & - & $17.78 \pm 0.04$ & $17.92 \pm 0.10$ & $17.77 \pm 0.15$ & 2 \\
\hline 010623.01-001456.2 & - & $\mathrm{DA}+\mathrm{M}$ & 20.834 & $14359 \pm 209$ & $7.5 \pm 0.045$ & $17.69 \pm 0.02$ & $17.22 \pm 0.02$ & $16.75 \pm 0.04$ & $16.47 \pm 0.04$ & 2 \\
\hline $010734.66+011157.0$ & - & $\mathrm{DA}+\mathrm{M}$ & 19.101 & $6301 \pm 3$ & $8.72 \pm 0.209$ & - & $17.22 \pm 0.02$ & $16.84 \pm 0.04$ & $16.55 \pm 0.05$ & 1 \\
\hline $011055.06+143922.3$ & $0108+143$ & DA & 17.037 & $9399 \pm 18$ & $9.0 \pm 0.0040$ & $16.77 \pm 0.01$ & $16.67 \pm 0.02$ & $16.63 \pm 0.03$ & $16.70 \pm 0.06$ & 2 \\
\hline $011634.11+002956.5$ & - & $\mathrm{DA}+\mathrm{M}$ & 18.942 & $6300 \pm 1$ & $8.53 \pm 0.286$ & $17.30 \pm 0.02$ & $16.79 \pm 0.02$ & $16.32 \pm 0.02$ & $15.99 \pm 0.03$ & 1 \\
\hline $011930.32+005329.6$ & - & DA & 19.200 & $7890 \pm 89$ & $8.89 \pm 0.11$ & - & $18.72 \pm 0.07$ & $18.42 \pm 0.19$ & $18.28 \pm 0.20$ & 1 \\
\hline $012032.27-001351.1$ & - & DA & 20.237 & $10487 \pm 194$ & $7.91 \pm 0.227$ & $19.25 \pm 0.08$ & $18.34 \pm 0.07$ & $17.82 \pm 0.07$ & $17.42 \pm 0.08$ & 1 \\
\hline $012234.68+003025.8$ & - & DA & 17.255 & $11798 \pm 47$ & $7.87 \pm 0.022$ & $17.11 \pm 0.01$ & $17.04 \pm 0.02$ & $17.14 \pm 0.04$ & $17.19 \pm 0.08$ & 1 \\
\hline $012851.81+151311.0$ & $0126+149$ & DA & 17.920 & $9650 \pm 41$ & $8.35 \pm 0.054$ & $17.66 \pm 0.03$ & $17.56 \pm 0.04$ & $17.49 \pm 0.06$ & $17.87 \pm 0.17$ & 2 \\
\hline $013256.41+133718.7$ & $0130+133$ & DA & 18.831 & $7679 \pm 83$ & $8.35 \pm 0.212$ & $18.44 \pm 0.05$ & $18.52 \pm 0.09$ & $18.13 \pm 0.09$ & $18.31 \pm 0.19$ & 2 \\
\hline $013328.62+142459.3$ & - & $\mathrm{DA}+\mathrm{M}$ & 19.548 & $41076 \pm 1506$ & $7.68 \pm 0.149$ & $16.38 \pm 0.01$ & $15.92 \pm 0.01$ & $15.29 \pm 0.01$ & $15.06 \pm 0.01$ & 1 \\
\hline $013335.55+130357.1$ & $0130+128$ & $\mathrm{DA}+\mathrm{M}$ & 18.812 & $6300 \pm 2$ & $8.68 \pm 0.237$ & $17.45 \pm 0.02$ & $16.95 \pm 0.02$ & $16.45 \pm 0.03$ & $16.14 \pm 0.03$ & 2 \\
\hline 013440.94-010902.3 & 0132-014 & DA & 18.270 & $10319 \pm 53$ & $8.12 \pm 0.066$ & $17.92 \pm 0.04$ & $17.97 \pm 0.05$ & $17.84 \pm 0.06$ & $17.76 \pm 0.12$ & 2 \\
\hline $013532.98+144555.8$ & $0132+145$ & DA & 16.960 & $8083 \pm 22$ & $8.08 \pm 0.043$ & $16.49 \pm 0.01$ & $16.24 \pm 0.01$ & $15.86 \pm 0.01$ & $15.56 \pm 0.02$ & 2 \\
\hline $013716.08+000311.3$ & - & $\mathrm{DA}+\mathrm{M}$ & 18.047 & $6300 \pm 1$ & $8.77 \pm 0.131$ & $16.73 \pm 0.01$ & $16.21 \pm 0.01$ & $15.61 \pm 0.01$ & $15.43 \pm 0.02$ & 3 \\
\hline 014009.00-001243.5 & - & $\mathrm{DA}+\mathrm{M}$ & 18.591 & $6304 \pm 9$ & $7.62 \pm 0.863$ & $17.30 \pm 0.02$ & $16.79 \pm 0.02$ & $16.27 \pm 0.02$ & $16.04 \pm 0.03$ & 3 \\
\hline
\end{tabular}
Continued on next page 
Table C.1- continued from previous page

\begin{tabular}{|c|c|c|c|c|c|c|c|c|c|c|}
\hline SDSS J & WD & Spec. Type & $i^{\prime}$ & $T_{\text {eff }}(\mathrm{K})$ & $\log g$ & $\bar{Y}$ & $\mathrm{~J}$ & $\mathrm{H}$ & $\bar{K}$ & Ref. \\
\hline $014342.45+131013.4$ & $0141+129$ & $\mathrm{DA}$ & 17.649 & $8355 \pm 19$ & $8.49 \pm 0.03$ & $17.22 \pm 0.02$ & $17.14 \pm 0.02$ & $17.08 \pm 0.05$ & $17.12 \pm 0.08$ & 2 \\
\hline $015259.18+010017.7$ & $0150+007$ & DA & 16.751 & $12494 \pm 71$ & $7.84 \pm 0.023$ & $16.54 \pm 0.01$ & $16.53 \pm 0.02$ & $16.65 \pm 0.04$ & $16.62 \pm 0.06$ & 2 \\
\hline $020343.21-002527.7$ & - & $\mathrm{DA}+\mathrm{M}$ & 19.134 & $6300 \pm 1$ & $8.61 \pm 0.3$ & $17.44 \pm 0.02$ & $16.90 \pm 0.02$ & $16.32 \pm 0.02$ & $15.99 \pm 0.02$ & 1 \\
\hline $020351.29+004025.0$ & $0201+004$ & $\mathrm{DA}+\mathrm{M}$ & 17.639 & $6300 \pm 1$ & $8.0 \pm 0.199$ & $16.15 \pm 0.01$ & $15.63 \pm 0.01$ & $15.11 \pm 0.01$ & $14.84 \pm 0.01$ & 2 \\
\hline $020611.79+132408.7$ & $0203+131$ & $\mathrm{DA}+\mathrm{M}$ & 18.809 & $10586 \pm 87$ & $8.28 \pm 0.075$ & $17.49 \pm 0.02$ & $16.91 \pm 0.02$ & $16.40 \pm 0.02$ & $16.13 \pm 0.03$ & 2 \\
\hline $020716.79+134744.3$ & $0204+135$ & DA & 19.230 & $8155 \pm 61$ & $8.3 \pm 0.121$ & $18.95 \pm 0.06$ & $18.75 \pm 0.08$ & $18.81 \pm 0.14$ & $18.17 \pm 0.19$ & 2 \\
\hline $020806.42+001833.8$ & $0205+000$ & $\mathrm{DA}+\mathrm{M}$ & 18.181 & $35368 \pm 223$ & $6.94 \pm 0.036$ & $12.79 \pm 0.00$ & $12.33 \pm 0.00$ & $11.73 \pm 0.00$ & $11.48 \pm 0.00$ & 2 \\
\hline $020851.68+005332.2$ & - & DA & 17.450 & $13401 \pm 150$ & $7.77 \pm 0.024$ & $17.33 \pm 0.02$ & $17.35 \pm 0.04$ & $17.28 \pm 0.06$ & $17.40 \pm 0.10$ & 1 \\
\hline 020952.78-000644.0 & - & DA & 18.389 & $9424 \pm 56$ & $8.34 \pm 0.081$ & $18.12 \pm 0.03$ & $18.11 \pm 0.05$ & $18.03 \pm 0.12$ & $18.41 \pm 0.25$ & 2 \\
\hline $021028.71+124318.9$ & $0207+124$ & DA & 17.473 & $17155 \pm 88$ & $7.9 \pm 0.017$ & $17.39 \pm 0.02$ & $17.45 \pm 0.03$ & $17.56 \pm 0.05$ & $17.59 \pm 0.11$ & 2 \\
\hline $021104.61+010615.6$ & - & DA & 19.323 & $7424 \pm 90$ & $8.8 \pm 0.199$ & $18.71 \pm 0.05$ & $18.70 \pm 0.10$ & $18.43 \pm 0.15$ & $18.15 \pm 0.18$ & 1 \\
\hline $021116.37+003128.2$ & $0208+002$ & DAH & 18.679 & $10161 \pm 90$ & $8.34 \pm 0.489$ & $18.34 \pm 0.04$ & $18.40 \pm 0.09$ & $18.15 \pm 0.13$ & $18.05 \pm 0.17$ & 2 \\
\hline $021239.46+001856.9$ & - & $\mathrm{DA}+\mathrm{M}$ & 17.954 & $6300 \pm 1$ & $7.3 \pm 0.214$ & $16.26 \pm 0.01$ & $15.71 \pm 0.01$ & $15.10 \pm 0.01$ & $14.84 \pm 0.01$ & 3 \\
\hline $021303.29+140232.8$ & $0210+138$ & $\mathrm{DA}+\mathrm{M}$ & 19.804 & $9081 \pm 167$ & $8.32 \pm 0.198$ & $18.43 \pm 0.04$ & $17.87 \pm 0.04$ & $17.39 \pm 0.04$ & $17.09 \pm 0.07$ & 2 \\
\hline 021309.19-005025.3 & - & $\mathrm{DA}+\mathrm{M}$ & 18.440 & $6300 \pm 1$ & $8.79 \pm 0.144$ & $16.91 \pm 0.01$ & $16.38 \pm 0.01$ & $15.84 \pm 0.01$ & $15.54 \pm 0.02$ & 2 \\
\hline $021326.76+001103.6$ & - & DA & 18.423 & $10485 \pm 44$ & $8.06 \pm 0.048$ & $18.19 \pm 0.03$ & $18.10 \pm 0.04$ & $17.98 \pm 0.11$ & $18.38 \pm 0.23$ & 1 \\
\hline $021411.35+011533.3$ & - & $\mathrm{DA}+\mathrm{M}$ & 17.993 & $6300 \pm 1$ & $8.48 \pm 0.236$ & $16.55 \pm 0.01$ & $16.06 \pm 0.01$ & $15.50 \pm 0.01$ & $15.28 \pm 0.01$ & 3 \\
\hline $021726.27-003317.9$ & - & $\mathrm{DA}+\mathrm{M}$ & 16.110 & $6300 \pm 1$ & $8.73 \pm 0.072$ & $14.70 \pm 0.00$ & $14.22 \pm 0.00$ & $13.58 \pm 0.00$ & $13.36 \pm 0.00$ & 1 \\
\hline $021742.44+005342.7$ & - & $\mathrm{DA}+\mathrm{M}$ & 16.560 & $6300 \pm 2$ & $8.73 \pm 0.066$ & $15.21 \pm 0.00$ & $14.71 \pm 0.00$ & $14.13 \pm 0.00$ & $13.90 \pm 0.00$ & 3 \\
\hline $021744.30+005824.0$ & $0215+007$ & DA & 18.004 & $13596 \pm 238$ & $7.94 \pm 0.04$ & $17.82 \pm 0.03$ & $17.92 \pm 0.06$ & $17.91 \pm 0.10$ & $17.87 \pm 0.14$ & 2 \\
\hline $021759.08+004517.6$ & - & $\mathrm{DA}+\mathrm{M}$ & 18.549 & $6300 \pm 1$ & $7.69 \pm 0.347$ & $16.91 \pm 0.02$ & $16.40 \pm 0.01$ & $15.87 \pm 0.02$ & $15.57 \pm 0.02$ & 3 \\
\hline $021837.76+003334.1$ & - & DA & 18.257 & $17659 \pm 118$ & $8.03 \pm 0.023$ & $18.05 \pm 0.04$ & $18.32 \pm 0.08$ & $18.50 \pm 0.18$ & $18.18 \pm 0.20$ & 1 \\
\hline $021849.98+005739.2$ & - & $\mathrm{DA}+\mathrm{M}$ & 18.271 & $6300 \pm 1$ & $7.14 \pm 0.17$ & $16.82 \pm 0.01$ & $16.34 \pm 0.01$ & $15.81 \pm 0.01$ & $15.52 \pm 0.02$ & 3 \\
\hline $021855.27+011056.3$ & - & $\mathrm{DA}+\mathrm{M}$ & 19.875 & $9756 \pm 106$ & $8.1 \pm 0.151$ & $18.41 \pm 0.04$ & $17.92 \pm 0.06$ & $17.56 \pm 0.07$ & $17.09 \pm 0.07$ & 3 \\
\hline $021903.67-001733.9$ & - & $\mathrm{DA}+\mathrm{M}$ & 18.073 & $6300 \pm 1$ & $7.79 \pm 0.238$ & $16.63 \pm 0.01$ & $16.13 \pm 0.01$ & $15.62 \pm 0.01$ & $15.43 \pm 0.02$ & 3 \\
\hline $021952.57-002249.0$ & - & DA & 17.006 & $20241 \pm 146$ & $7.87 \pm 0.024$ & $16.87 \pm 0.01$ & $16.90 \pm 0.02$ & $16.93 \pm 0.03$ & $17.10 \pm 0.09$ & 2 \\
\hline $022036.11+001237.9$ & - & DA & 19.247 & $7950 \pm 109$ & $8.66 \pm 0.266$ & $18.80 \pm 0.06$ & $18.80 \pm 0.10$ & $18.63 \pm 0.19$ & $18.20 \pm 0.22$ & 1 \\
\hline $022108.68+004924.5$ & $0218+005$ & DA & 18.860 & $10608 \pm 65$ & $8.21 \pm 0.06$ & $18.52 \pm 0.05$ & $18.61 \pm 0.12$ & $18.42 \pm 0.16$ & $18.32 \pm 0.22$ & 2 \\
\hline $022125.36+001710.4$ & - & $\mathrm{DA}+\mathrm{M}$ & 19.531 & $6301 \pm 4$ & $8.3 \pm 0.479$ & $18.10 \pm 0.04$ & $17.69 \pm 0.04$ & $17.26 \pm 0.05$ & $17.02 \pm 0.07$ & 1 \\
\hline $023015.36+010958.8$ & - & $\mathrm{DA}+\mathrm{M}$ & 19.865 & $9375 \pm 183$ & $8.2 \pm 0.251$ & $18.30 \pm 0.04$ & $17.72 \pm 0.04$ & $17.36 \pm 0.06$ & $16.91 \pm 0.07$ & 1 \\
\hline $023113.02-004057.1$ & - & $\mathrm{DA}+\mathrm{M}$ & 19.266 & $6305 \pm 10$ & $7.34 \pm 0.561$ & - & $17.31 \pm 0.03$ & $16.79 \pm 0.04$ & $16.59 \pm 0.06$ & 3 \\
\hline 023247.50-003909.3 & - & DA & 20.121 & $9898 \pm 161$ & $8.04 \pm 0.238$ & - & $20.24 \pm 0.34$ & $18.80 \pm 0.20$ & $17.84 \pm 0.16$ & 1 \\
\hline
\end{tabular}

Continued on next page 
Table C.1- continued from previous page

\begin{tabular}{|c|c|c|c|c|c|c|c|c|c|c|}
\hline SDSS J & WD & Spec. Type & $i^{\prime}$ & $T_{\text {eff }}(\mathrm{K})$ & $\log g$ & $\bar{Y}$ & $\mathrm{~J}$ & $\mathrm{H}$ & $\bar{K}$ & Ref. \\
\hline $023543.09+005557.1$ & $0233+007$ & $\overline{\mathrm{DA}}$ & 18.250 & $10184 \pm 47$ & $8.32 \pm 0.057$ & $17.94 \pm 0.03$ & $17.90 \pm 0.05$ & $17.76 \pm 0.09$ & $17.77 \pm 0.12$ & 2 \\
\hline 023650.61-010313.2 & 0234-012 & $\mathrm{DA}+\mathrm{M}$ & 18.795 & $19968 \pm 253$ & $7.54 \pm 0.047$ & - & $17.70 \pm 0.04$ & $17.23 \pm 0.05$ & $17.01 \pm 0.08$ & 2 \\
\hline 023804.39-000545.7 & - & $\mathrm{DA}+\mathrm{M}$ & 17.698 & $6300 \pm 1$ & $8.18 \pm 0.235$ & $16.09 \pm 0.01$ & $15.55 \pm 0.01$ & $15.05 \pm 0.01$ & $14.73 \pm 0.01$ & 3 \\
\hline $024350.05-005000.8$ & - & $\mathrm{DA}$ & 18.860 & $8180 \pm 69$ & $8.61 \pm 0.131$ & $18.42 \pm 0.05$ & $18.38 \pm 0.08$ & $18.20 \pm 0.12$ & $18.45 \pm 0.23$ & 2 \\
\hline $024449.20-002737.7$ & - & DA & 18.579 & $8496 \pm 54$ & $8.48 \pm 0.109$ & $18.21 \pm 0.04$ & $18.06 \pm 0.06$ & $18.23 \pm 0.12$ & $18.64 \pm 0.28$ & 2 \\
\hline $024602.69+002539.2$ & $0243+002$ & DA & 17.745 & $14441 \pm 116$ & $7.83 \pm 0.024$ & $17.63 \pm 0.02$ & - & $17.74 \pm 0.06$ & $17.91 \pm 0.12$ & 2 \\
\hline $024642.56+004137.1$ & $0244+004$ & $\mathrm{DA}+\mathrm{M}$ & 17.292 & $6300 \pm 1$ & $8.74 \pm 0.108$ & $15.73 \pm 0.01$ & $15.15 \pm 0.00$ & $14.58 \pm 0.00$ & $14.30 \pm 0.01$ & 2 \\
\hline $024746.33+000331.4$ & $0245-001$ & DA & 16.913 & $19084 \pm 108$ & $8.04 \pm 0.019$ & $16.81 \pm 0.01$ & - & $16.84 \pm 0.04$ & $16.88 \pm 0.07$ & 1 \\
\hline 025123.33-011314.4 & - & $\mathrm{DA}+\mathrm{M}$ & 18.758 & $36285 \pm 392$ & $7.63 \pm 0.074$ & $17.63 \pm 0.02$ & $17.18 \pm 0.03$ & $16.68 \pm 0.03$ & $16.38 \pm 0.04$ & 3 \\
\hline $025129.08+002749.3$ & $0248+002$ & DA & 18.971 & $7835 \pm 79$ & $8.91 \pm 0.096$ & $18.51 \pm 0.04$ & - & $18.14 \pm 0.12$ & $18.04 \pm 0.19$ & 2 \\
\hline $025141.92-004129.8$ & - & $\mathrm{DA}+\mathrm{M}$ & 16.599 & $6300 \pm 2$ & $8.01 \pm 0.037$ & $14.96 \pm 0.00$ & $14.46 \pm 0.00$ & $13.92 \pm 0.00$ & $13.67 \pm 0.00$ & 3 \\
\hline $025147.85-000003.2$ & - & $\mathrm{DA}+\mathrm{M}$ & 19.141 & $6300 \pm 2$ & $8.61 \pm 0.29$ & $17.46 \pm 0.02$ & - & $16.36 \pm 0.03$ & $16.06 \pm 0.03$ & 1 \\
\hline $025200.99+004544.2$ & $0249+005$ & DA & 18.570 & $10180 \pm 64$ & $8.27 \pm 0.091$ & $18.44 \pm 0.04$ & $18.28 \pm 0.06$ & $18.17 \pm 0.09$ & $18.09 \pm 0.17$ & 2 \\
\hline $025304.77+001344.8$ & $0250+000$ & DA & 17.241 & $8094 \pm 32$ & $8.44 \pm 0.061$ & $16.87 \pm 0.01$ & - & $16.65 \pm 0.03$ & $16.53 \pm 0.06$ & 1 \\
\hline $025306.37+001329.3$ & - & $\mathrm{DA}+\mathrm{M}$ & 19.331 & $36000 \pm 540$ & $6.81 \pm 0.081$ & $14.60 \pm 0.00$ & - & $13.55 \pm 0.00$ & $13.26 \pm 0.00$ & 3 \\
\hline $025509.30-004414.9$ & - & $\mathrm{DA}+\mathrm{M}$ & 17.814 & $6300 \pm 1$ & $8.71 \pm 0.15$ & $16.39 \pm 0.01$ & $15.93 \pm 0.01$ & $15.30 \pm 0.01$ & $15.07 \pm 0.01$ & 3 \\
\hline $025709.00+004627.9$ & $0254+005$ & DA & 17.783 & $12215 \pm 83$ & $8.01 \pm 0.033$ & $17.65 \pm 0.02$ & $17.69 \pm 0.02$ & $17.70 \pm 0.05$ & $17.64 \pm 0.08$ & 2 \\
\hline $025746.40+010106.1$ & $0255+008$ & DA & 18.203 & $16579 \pm 213$ & $8.29 \pm 0.037$ & $18.24 \pm 0.04$ & $18.27 \pm 0.05$ & $18.22 \pm 0.09$ & $18.26 \pm 0.16$ & 2 \\
\hline $025747.80+004902.2$ & - & $\mathrm{DA}+\mathrm{M}$ & 18.161 & $6300 \pm 1$ & $8.63 \pm 0.173$ & $16.39 \pm 0.01$ & $15.81 \pm 0.01$ & $15.26 \pm 0.01$ & $14.93 \pm 0.01$ & 3 \\
\hline $025753.40-003159.6$ & - & $\mathrm{DA}+\mathrm{M}$ & 18.968 & $9490 \pm 109$ & $8.02 \pm 0.151$ & $17.51 \pm 0.02$ & $17.00 \pm 0.03$ & $16.57 \pm 0.02$ & $16.31 \pm 0.03$ & 2 \\
\hline $025801.20-005400.1$ & - & DA & 18.162 & $9431 \pm 48$ & $8.17 \pm 0.087$ & $17.83 \pm 0.03$ & $17.78 \pm 0.05$ & $17.73 \pm 0.05$ & $17.66 \pm 0.09$ & 2 \\
\hline $025817.88+010945.9$ & $0255+009.2$ & $\mathrm{DA}+\mathrm{M}$ & 17.377 & $6632 \pm 60$ & $7.73 \pm 0.162$ & $15.95 \pm 0.01$ & $15.46 \pm 0.01$ & $14.82 \pm 0.01$ & $14.56 \pm 0.01$ & 2 \\
\hline 025935.27-011405.1 & - & DA & 18.088 & $51639 \pm 1005$ & $7.03 \pm 0.084$ & $18.05 \pm 0.03$ & $18.31 \pm 0.09$ & $18.25 \pm 0.09$ & $18.29 \pm 0.16$ & 1 \\
\hline $030138.75+004026.1$ & - & $\mathrm{DA}+\mathrm{M}$ & 19.812 & $6321 \pm 28$ & $7.56 \pm 0.672$ & $18.79 \pm 0.06$ & $18.38 \pm 0.05$ & $17.91 \pm 0.10$ & $17.76 \pm 0.11$ & 1 \\
\hline $030335.08+000221.5$ & 0301-001 & DA & 17.857 & $8567 \pm 29$ & $8.53 \pm 0.045$ & $17.49 \pm 0.02$ & - & $17.36 \pm 0.04$ & $17.35 \pm 0.07$ & 2 \\
\hline $030342.22+005310.3$ & - & DA & 18.358 & $13501 \pm 192$ & $7.89 \pm 0.031$ & $18.23 \pm 0.03$ & $18.24 \pm 0.06$ & $18.29 \pm 0.11$ & $18.06 \pm 0.18$ & 1 \\
\hline $030351.96+003548.4$ & $0301+004$ & $\mathrm{DA}+\mathrm{M}$ & 18.067 & $6300 \pm 1$ & $8.79 \pm 0.126$ & $16.75 \pm 0.01$ & $16.28 \pm 0.01$ & $15.77 \pm 0.01$ & $15.54 \pm 0.02$ & 2 \\
\hline $030407.39-002541.9$ & - & DAH & 18.130 & $23109 \pm 307$ & $9.0 \pm 0.0010$ & $17.98 \pm 0.03$ & $18.02 \pm 0.06$ & $18.02 \pm 0.06$ & $18.08 \pm 0.13$ & 2 \\
\hline $030607.18-003114.3$ & - & $\mathrm{DA}+\mathrm{M}$ & 15.294 & $20870 \pm 112$ & $7.26 \pm 0.022$ & $13.70 \pm 0.00$ & $13.16 \pm 0.00$ & $12.65 \pm 0.00$ & $12.39 \pm 0.00$ & 3 \\
\hline $030859.86-002735.8$ & - & $\mathrm{DA}+\mathrm{M}$ & 17.797 & $6300 \pm 1$ & $8.33 \pm 0.286$ & $16.21 \pm 0.01$ & $15.65 \pm 0.01$ & $15.09 \pm 0.01$ & $14.82 \pm 0.01$ & 3 \\
\hline $030904.82-010100.9$ & - & $\mathrm{DA}+\mathrm{M}$ & 18.429 & $6300 \pm 1$ & $8.74 \pm 0.162$ & $16.88 \pm 0.01$ & $16.34 \pm 0.01$ & $15.85 \pm 0.01$ & $15.60 \pm 0.02$ & 3 \\
\hline 030924.29-004504.3 & - & DA & 19.300 & $7375 \pm 107$ & $8.88 \pm 0.128$ & $18.71 \pm 0.06$ & $18.74 \pm 0.13$ & $18.42 \pm 0.09$ & $18.53 \pm 0.22$ & 1 \\
\hline
\end{tabular}

Continued on next page 
Table C.1- continued from previous page

\begin{tabular}{|c|c|c|c|c|c|c|c|c|c|c|}
\hline SDSS J & WD & Spec. Type & $i^{\prime}$ & $T_{\text {eff }}(\mathrm{K})$ & $\log g$ & $\bar{Y}$ & $\mathrm{~J}$ & $\mathrm{H}$ & $\bar{K}$ & Ref. \\
\hline $031301.42+010558.1$ & $0310+009$ & $\mathrm{DA}$ & 18.740 & $8663 \pm 64$ & $8.34 \pm 0.141$ & $18.34 \pm 0.03$ & $18.31 \pm 0.04$ & $18.15 \pm 0.10$ & $18.45 \pm 0.17$ & 2 \\
\hline $031325.91+010329.4$ & - & DA & 19.226 & $8627 \pm 80$ & $8.11 \pm 0.149$ & $18.95 \pm 0.05$ & $18.82 \pm 0.06$ & $18.61 \pm 0.15$ & $18.67 \pm 0.21$ & 1 \\
\hline 031404.98-011136.6 & - & $\mathrm{DA}+\mathrm{M}$ & 17.773 & $6300 \pm 1$ & $8.88 \pm 0.096$ & $16.01 \pm 0.01$ & $15.44 \pm 0.01$ & $14.88 \pm 0.01$ & $14.58 \pm 0.01$ & 3 \\
\hline $031553.09+011157.5$ & - & $\mathrm{DA}$ & 19.374 & $7962 \pm 119$ & $7.69 \pm 0.303$ & $18.88 \pm 0.05$ & $18.77 \pm 0.06$ & $18.67 \pm 0.15$ & $18.72 \pm 0.22$ & 1 \\
\hline $031737.31+005033.4$ & - & DA & 18.742 & $8010 \pm 66$ & $8.23 \pm 0.156$ & $18.26 \pm 0.03$ & $18.20 \pm 0.04$ & $17.97 \pm 0.09$ & $18.16 \pm 0.13$ & 1 \\
\hline $031957.78-005803.3$ & - & $\mathrm{DA}+\mathrm{M}$ & 20.029 & $10392 \pm 163$ & $8.29 \pm 0.18$ & $19.04 \pm 0.06$ & $18.57 \pm 0.05$ & $18.01 \pm 0.11$ & $18.00 \pm 0.18$ & 2 \\
\hline $032150.57+005312.4$ & - & DA & 18.028 & $8842 \pm 23$ & $8.34 \pm 0.046$ & $17.72 \pm 0.02$ & $17.66 \pm 0.02$ & $17.61 \pm 0.07$ & $17.58 \pm 0.07$ & 1 \\
\hline $032317.00-002612.7$ & - & DA & 20.775 & $22193 \pm 1446$ & $7.1 \pm 0.193$ & $15.03 \pm 0.00$ & $14.63 \pm 0.00$ & $14.08 \pm 0.00$ & $13.81 \pm 0.01$ & 1 \\
\hline 032443.01-002956.1 & - & DA & 18.364 & $8200 \pm 36$ & $8.35 \pm 0.084$ & $18.04 \pm 0.03$ & $17.89 \pm 0.03$ & $17.83 \pm 0.08$ & $17.73 \pm 0.13$ & 2 \\
\hline $032510.84-011114.2$ & - & $\mathrm{DA}+\mathrm{M}$ & 17.501 & $18221 \pm 105$ & $7.65 \pm 0.021$ & $12.74 \pm 0.00$ & $12.30 \pm 0.00$ & $11.84 \pm 0.00$ & $11.59 \pm 0.00$ & 3 \\
\hline $032619.44+001817.3$ & $0323+001$ & DA & 17.868 & $12124 \pm 58$ & $8.07 \pm 0.023$ & $17.67 \pm 0.02$ & $17.66 \pm 0.02$ & $17.65 \pm 0.04$ & $17.57 \pm 0.11$ & 2 \\
\hline $032643.22-010338.0$ & - & $\mathrm{DA}+\mathrm{M}$ & 18.486 & $6300 \pm 2$ & $7.93 \pm 0.392$ & $17.04 \pm 0.01$ & $16.53 \pm 0.01$ & $15.99 \pm 0.02$ & $15.72 \pm 0.02$ & 1 \\
\hline 032706.09-010538.6 & - & $\mathrm{DA}+\mathrm{M}$ & 20.266 & $8601 \pm 338$ & $7.78 \pm 0.621$ & $18.72 \pm 0.05$ & $18.21 \pm 0.04$ & $17.72 \pm 0.08$ & $17.30 \pm 0.09$ & 1 \\
\hline $032727.52+001252.5$ & - & DA & 18.193 & $15575 \pm 322$ & $8.53 \pm 0.029$ & $17.96 \pm 0.02$ & $18.03 \pm 0.03$ & $17.96 \pm 0.05$ & $17.95 \pm 0.16$ & 1 \\
\hline $032758.15-002215.4$ & - & $\mathrm{DA}+\mathrm{M}$ & 17.453 & $6300 \pm 1$ & $7.24 \pm 0.155$ & $15.94 \pm 0.01$ & $15.42 \pm 0.00$ & $14.91 \pm 0.01$ & $14.62 \pm 0.01$ & 2 \\
\hline $033132.13-005453.3$ & - & $\mathrm{DA}+\mathrm{M}$ & 17.056 & $7589 \pm 39$ & $8.17 \pm 0.071$ & $15.63 \pm 0.01$ & $15.08 \pm 0.00$ & $14.51 \pm 0.01$ & $14.26 \pm 0.01$ & 3 \\
\hline $033133.89+010327.8$ & $0328+008$ & DA & 17.245 & $36000 \pm 77$ & $8.01 \pm 0.033$ & $17.18 \pm 0.02$ & $17.30 \pm 0.02$ & $17.31 \pm 0.07$ & $17.37 \pm 0.08$ & 2 \\
\hline $033145.69+004517.0$ & $0329+005$ & DAH & 17.701 & $29150 \pm 169$ & $9.0 \pm 0.0020$ & $17.67 \pm 0.02$ & $17.71 \pm 0.02$ & $17.77 \pm 0.10$ & $17.84 \pm 0.10$ & 2 \\
\hline $033200.48-005752.5$ & - & DA & 17.621 & $17476 \pm 109$ & $7.77 \pm 0.023$ & $17.52 \pm 0.02$ & $17.54 \pm 0.02$ & $17.49 \pm 0.07$ & $17.64 \pm 0.13$ & 2 \\
\hline 033236.60-004918.4 & - & DA & 18.441 & $11041 \pm 68$ & $8.25 \pm 0.057$ & - & $18.13 \pm 0.05$ & $18.27 \pm 0.13$ & $18.08 \pm 0.18$ & 2 \\
\hline $033236.86-004936.9$ & - & DA & 16.392 & $35493 \pm 50$ & $7.87 \pm 0.025$ & - & $16.48 \pm 0.01$ & $16.57 \pm 0.03$ & $16.77 \pm 0.05$ & 1 \\
\hline 033432.24-010600.9 & - & DA & 18.546 & $6563 \pm 87$ & $8.89 \pm 0.114$ & - & $17.77 \pm 0.04$ & $17.58 \pm 0.07$ & $17.65 \pm 0.14$ & 1 \\
\hline $033548.57+003832.1$ & $0333+004$ & $\mathrm{DA}+\mathrm{M}$ & 17.134 & $8617 \pm 29$ & $8.24 \pm 0.041$ & $15.54 \pm 0.01$ & $15.02 \pm 0.00$ & $14.49 \pm 0.01$ & $14.19 \pm 0.01$ & 2 \\
\hline $033648.34-000634.3$ & - & DA & 18.186 & $10396 \pm 43$ & $8.26 \pm 0.044$ & $17.98 \pm 0.02$ & $17.88 \pm 0.03$ & $17.93 \pm 0.06$ & $18.01 \pm 0.18$ & 2 \\
\hline $033807.63-000715.2$ & - & $\mathrm{DA}+\mathrm{M}$ & 18.560 & $6301 \pm 3$ & $7.76 \pm 0.408$ & $17.31 \pm 0.02$ & $16.80 \pm 0.01$ & $16.25 \pm 0.01$ & $15.94 \pm 0.03$ & 3 \\
\hline $034000.84-004257.1$ & - & DA & 18.830 & $9996 \pm 54$ & $8.04 \pm 0.08$ & - & $18.63 \pm 0.10$ & $18.44 \pm 0.17$ & $18.25 \pm 0.24$ & 2 \\
\hline 034038.76-002945.2 & - & DA & 17.015 & $7719 \pm 25$ & $8.55 \pm 0.054$ & - & $16.42 \pm 0.01$ & $16.32 \pm 0.03$ & $16.30 \pm 0.04$ & 2 \\
\hline $034221.55+005345.6$ & - & DApec & 19.760 & $23035 \pm 684$ & $6.97 \pm 0.077$ & $16.48 \pm 0.01$ & $16.05 \pm 0.01$ & $15.45 \pm 0.01$ & $15.31 \pm 0.01$ & 1 \\
\hline 034409.68-001358.2 & - & DA & 17.984 & $9097 \pm 30$ & $8.1 \pm 0.054$ & $17.67 \pm 0.02$ & $17.54 \pm 0.03$ & $17.59 \pm 0.05$ & $17.56 \pm 0.12$ & 2 \\
\hline $034428.27-003814.0$ & - & DA & 17.546 & $30000 \pm 71$ & $7.76 \pm 0.026$ & - & $17.67 \pm 0.03$ & $17.66 \pm 0.06$ & $17.71 \pm 0.13$ & 2 \\
\hline $034511.09+003444.1$ & $0342+004$ & DAH & 18.508 & $7380 \pm 48$ & $8.99 \pm 0.021$ & $18.06 \pm 0.03$ & $18.01 \pm 0.04$ & $17.81 \pm 0.09$ & $17.70 \pm 0.09$ & 2 \\
\hline $035256.11-000841.7$ & - & $\mathrm{DA}+\mathrm{M}$ & 19.481 & $8459 \pm 243$ & $8.94 \pm 0.071$ & $18.34 \pm 0.03$ & $17.97 \pm 0.04$ & $17.18 \pm 0.03$ & $16.82 \pm 0.04$ & 1 \\
\hline
\end{tabular}

Continued on next page 
Table C.1- continued from previous page

\begin{tabular}{|c|c|c|c|c|c|c|c|c|c|c|}
\hline SDSS J & WD & Spec. Type & $i^{\prime}$ & $T_{\text {eff }}(\mathrm{K})$ & $\log g$ & $\bar{Y}$ & $\mathrm{~J}$ & $\mathrm{H}$ & $\bar{K}$ & Ref. \\
\hline $081922.84+031809.6$ & - & $\overline{D A}$ & 17.919 & $16947 \pm 148$ & $8.19 \pm 0.029$ & - & - & $17.85 \pm 0.08$ & $18.03 \pm 0.15$ & 1 \\
\hline $082239.43+082436.7$ & - & DA & 18.269 & $11205 \pm 70$ & $8.52 \pm 0.044$ & $18.09 \pm 0.03$ & $18.01 \pm 0.03$ & $17.87 \pm 0.05$ & $18.09 \pm 0.13$ & 1 \\
\hline $082337.65+033554.0$ & - & DA & 17.708 & $19251 \pm 162$ & $7.92 \pm 0.028$ & - & - & $17.62 \pm 0.07$ & $17.62 \pm 0.11$ & 1 \\
\hline $082518.86+032927.8$ & - & DA & 17.871 & $11801 \pm 105$ & $8.33 \pm 0.044$ & - & - & $17.72 \pm 0.07$ & $17.68 \pm 0.11$ & 1 \\
\hline $082558.77+034520.8$ & - & $\mathrm{DA}+\mathrm{M}$ & 19.627 & $10108 \pm 136$ & $8.82 \pm 0.128$ & - & - & $17.70 \pm 0.06$ & $17.57 \pm 0.10$ & 1 \\
\hline $082936.62+072824.6$ & - & DA & 19.270 & $11481 \pm 149$ & $7.98 \pm 0.088$ & $19.04 \pm 0.06$ & $19.07 \pm 0.10$ & $19.04 \pm 0.17$ & $18.98 \pm 0.25$ & 1 \\
\hline $083005.06+042347.7$ & - & DA & 18.679 & $9387 \pm 77$ & $8.87 \pm 0.115$ & $18.36 \pm 0.04$ & $18.31 \pm 0.04$ & $18.42 \pm 0.11$ & $18.54 \pm 0.23$ & 1 \\
\hline $083354.84+070240.1$ & - & $\mathrm{DA}+\mathrm{M}$ & 18.148 & $9084 \pm 62$ & $7.86 \pm 0.1$ & $16.78 \pm 0.01$ & $16.25 \pm 0.01$ & $15.75 \pm 0.01$ & $15.47 \pm 0.01$ & 3 \\
\hline $083357.02+052901.1$ & - & $\mathrm{DA}+\mathrm{M}$ & 19.485 & $6303 \pm 8$ & $7.04 \pm 0.407$ & $17.76 \pm 0.02$ & $17.22 \pm 0.02$ & $16.63 \pm 0.02$ & $16.31 \pm 0.03$ & 3 \\
\hline $083621.87+044624.3$ & - & $\mathrm{DA}+\mathrm{M}$ & 19.886 & $8560 \pm 172$ & $8.89 \pm 0.108$ & $18.45 \pm 0.04$ & $18.02 \pm 0.03$ & $17.31 \pm 0.05$ & $17.09 \pm 0.06$ & 1 \\
\hline $083736.13+075107.2$ & - & DA & 17.875 & $17149 \pm 99$ & $7.88 \pm 0.02$ & $17.80 \pm 0.03$ & $17.84 \pm 0.04$ & $17.91 \pm 0.08$ & $17.91 \pm 0.10$ & 1 \\
\hline $084001.80+051628.0$ & - & DA & 18.026 & $8124 \pm 37$ & $8.39 \pm 0.065$ & $17.68 \pm 0.02$ & $17.52 \pm 0.02$ & $17.38 \pm 0.04$ & $17.48 \pm 0.08$ & 1 \\
\hline $084052.72+024712.3$ & - & DA & 18.625 & $7762 \pm 49$ & $8.6 \pm 0.124$ & $18.18 \pm 0.03$ & $18.08 \pm 0.05$ & $18.01 \pm 0.08$ & $17.76 \pm 0.14$ & 1 \\
\hline $084141.63+051117.0$ & - & DA & 18.756 & $8085 \pm 61$ & $8.61 \pm 0.117$ & $18.30 \pm 0.03$ & $18.21 \pm 0.04$ & $18.00 \pm 0.07$ & $18.14 \pm 0.17$ & 1 \\
\hline $084147.52+082537.6$ & - & $\mathrm{DA}+\mathrm{M}$ & 18.571 & $6300 \pm 2$ & $8.73 \pm 0.195$ & $17.38 \pm 0.01$ & $16.89 \pm 0.02$ & $16.29 \pm 0.01$ & $16.09 \pm 0.02$ & 3 \\
\hline $084155.73+022350.5$ & - & DAH & 18.735 & $6668 \pm 49$ & $8.99 \pm 0.021$ & $18.33 \pm 0.03$ & $18.12 \pm 0.04$ & $18.03 \pm 0.07$ & $18.38 \pm 0.20$ & 1 \\
\hline $084314.05+043131.6$ & - & DA & 18.138 & $11250 \pm 63$ & $8.18 \pm 0.044$ & $17.95 \pm 0.03$ & $17.95 \pm 0.03$ & $17.88 \pm 0.06$ & $18.34 \pm 0.18$ & 1 \\
\hline $084400.81+052305.7$ & - & $\mathrm{DA}+\mathrm{M}$ & 18.971 & $6300 \pm 2$ & $8.7 \pm 0.227$ & $17.45 \pm 0.02$ & $16.87 \pm 0.01$ & $16.37 \pm 0.01$ & $16.12 \pm 0.03$ & 3 \\
\hline $084537.73+065346.2$ & - & DA & 17.254 & $22086 \pm 145$ & $8.43 \pm 0.02$ & $17.26 \pm 0.02$ & $17.31 \pm 0.02$ & $17.40 \pm 0.07$ & $17.63 \pm 0.10$ & 1 \\
\hline $084554.60-003206.5$ & - & DA & 18.353 & $15662 \pm 165$ & $7.92 \pm 0.037$ & $18.23 \pm 0.03$ & $18.37 \pm 0.06$ & $18.24 \pm 0.12$ & $18.19 \pm 0.18$ & 2 \\
\hline $084608.19+053818.0$ & - & DA & 18.099 & $8124 \pm 41$ & $9.0 \pm 0.0060$ & $17.88 \pm 0.02$ & $17.78 \pm 0.02$ & $17.71 \pm 0.05$ & $17.76 \pm 0.11$ & 1 \\
\hline $084654.44+051402.9$ & - & DA & 18.171 & $7231 \pm 51$ & $8.94 \pm 0.073$ & $17.71 \pm 0.02$ & $17.45 \pm 0.03$ & $17.31 \pm 0.04$ & $17.20 \pm 0.07$ & 1 \\
\hline $084833.59+005840.0$ & $0845+011$ & $\mathrm{DA}+\mathrm{M}$ & 17.611 & $6300 \pm 1$ & $7.0 \pm 0.103$ & $16.12 \pm 0.01$ & $15.59 \pm 0.01$ & $15.08 \pm 0.01$ & $14.76 \pm 0.01$ & 2 \\
\hline $084949.62+092353.3$ & - & DA & 17.796 & $8736 \pm 30$ & $8.38 \pm 0.047$ & $17.42 \pm 0.02$ & $17.33 \pm 0.02$ & $17.23 \pm 0.04$ & $17.25 \pm 0.06$ & 1 \\
\hline $085008.69+085450.6$ & - & DA & 18.994 & $11183 \pm 119$ & $8.37 \pm 0.076$ & $18.81 \pm 0.07$ & $18.80 \pm 0.07$ & $18.69 \pm 0.15$ & $18.83 \pm 0.23$ & 1 \\
\hline $085107.29+033046.0$ & - & $\mathrm{DA}+\mathrm{M}$ & 17.951 & $22291 \pm 227$ & $7.4 \pm 0.033$ & $17.23 \pm 0.02$ & - & $16.40 \pm 0.02$ & $16.05 \pm 0.03$ & 3 \\
\hline $085128.17+060551.1$ & - & DA & 17.114 & $11306 \pm 48$ & $8.11 \pm 0.029$ & $16.96 \pm 0.01$ & $16.93 \pm 0.01$ & $16.91 \pm 0.03$ & $17.01 \pm 0.05$ & 1 \\
\hline $085208.84+064155.3$ & - & $\mathrm{DA}+\mathrm{M}$ & 20.212 & $8580 \pm 229$ & $8.3 \pm 0.364$ & $18.76 \pm 0.06$ & $18.35 \pm 0.05$ & $17.75 \pm 0.08$ & $17.47 \pm 0.07$ & 1 \\
\hline $085216.70+065931.6$ & - & DA & 17.967 & $7677 \pm 56$ & $8.77 \pm 0.14$ & $17.57 \pm 0.02$ & $17.47 \pm 0.02$ & $17.35 \pm 0.05$ & $17.29 \pm 0.07$ & 1 \\
\hline $085418.66+014451.2$ & $0851+019$ & DA & 18.886 & $8346 \pm 64$ & $8.52 \pm 0.14$ & $18.57 \pm 0.03$ & $18.49 \pm 0.07$ & $18.29 \pm 0.13$ & $18.39 \pm 0.21$ & 2 \\
\hline $085507.29+063540.9$ & - & DA & 17.377 & $11045 \pm 51$ & $8.43 \pm 0.03$ & $17.31 \pm 0.02$ & $17.30 \pm 0.02$ & $17.20 \pm 0.04$ & $17.12 \pm 0.05$ & 1 \\
\hline $085529.22+052405.1$ & - & DA & 18.804 & $9672 \pm 71$ & $8.32 \pm 0.097$ & $18.55 \pm 0.04$ & $18.42 \pm 0.05$ & $18.26 \pm 0.11$ & $18.39 \pm 0.18$ & 1 \\
\hline
\end{tabular}

Continued on next page 
Table C.1- continued from previous page

\begin{tabular}{|c|c|c|c|c|c|c|c|c|c|c|}
\hline SDSS J & WD & Spec. Type & $i^{\prime}$ & $T_{\text {eff }}(\mathrm{K})$ & $\log g$ & $\bar{Y}$ & $\mathrm{~J}$ & $\mathrm{H}$ & $\bar{K}$ & Ref. \\
\hline $085552.42+084901.1$ & - & $\overline{\mathrm{DA}}$ & 19.132 & $9569 \pm 87$ & $8.35 \pm 0.152$ & $18.86 \pm 0.09$ & $18.83 \pm 0.07$ & $18.67 \pm 0.15$ & $18.45 \pm 0.18$ & 1 \\
\hline $085557.46+053524.5$ & - & $\mathrm{DA}+\mathrm{M}$ & 19.170 & $6304 \pm 9$ & $7.4 \pm 0.521$ & $17.67 \pm 0.02$ & $17.12 \pm 0.02$ & $16.55 \pm 0.02$ & $16.22 \pm 0.03$ & 3 \\
\hline $085557.48+081706.5$ & - & DA & 18.339 & $17219 \pm 118$ & $7.94 \pm 0.023$ & $18.26 \pm 0.03$ & $18.21 \pm 0.04$ & $18.22 \pm 0.06$ & $18.20 \pm 0.16$ & 1 \\
\hline $085616.67+064632.3$ & - & $\mathrm{DA}+\mathrm{M}$ & 19.289 & $7423 \pm 137$ & $7.36 \pm 0.354$ & $17.87 \pm 0.03$ & $17.35 \pm 0.02$ & $16.86 \pm 0.04$ & $16.52 \pm 0.03$ & 1 \\
\hline $085746.18+034255.3$ & - & DA & 18.386 & $36181 \pm 390$ & $7.17 \pm 0.08$ & - & - & $18.62 \pm 0.16$ & $18.14 \pm 0.17$ & 1 \\
\hline $085911.28+043507.1$ & - & $\mathrm{DA}+\mathrm{M}$ & 18.199 & $6300 \pm 2$ & $6.99 \pm 0.152$ & - & - & $15.74 \pm 0.01$ & $15.48 \pm 0.02$ & 3 \\
\hline $085956.47+082607.5$ & - & DA & 19.132 & $15623 \pm 237$ & $7.44 \pm 0.056$ & $18.33 \pm 0.03$ & - & $17.49 \pm 0.03$ & $17.09 \pm 0.08$ & 1 \\
\hline $090005.56+061535.3$ & - & DA & 17.901 & $18729 \pm 153$ & $7.9 \pm 0.027$ & $17.86 \pm 0.02$ & $17.90 \pm 0.02$ & $17.95 \pm 0.09$ & $17.88 \pm 0.10$ & 1 \\
\hline $090059.85+062920.5$ & - & DA & 18.367 & $24069 \pm 368$ & $7.88 \pm 0.041$ & $18.30 \pm 0.03$ & $18.33 \pm 0.03$ & $18.38 \pm 0.14$ & $18.38 \pm 0.17$ & 1 \\
\hline $090139.96+060547.3$ & - & DA & 18.889 & $10705 \pm 93$ & $8.1 \pm 0.083$ & $18.68 \pm 0.05$ & $18.67 \pm 0.08$ & $18.50 \pm 0.15$ & $18.78 \pm 0.24$ & 1 \\
\hline $090150.74+091211.3$ & - & DA & 18.707 & $7732 \pm 47$ & $9.0 \pm 0.0060$ & $18.48 \pm 0.06$ & $18.34 \pm 0.05$ & $18.08 \pm 0.06$ & $18.45 \pm 0.15$ & 1 \\
\hline $090348.56+083456.5$ & - & DA & 18.492 & $17860 \pm 146$ & $7.97 \pm 0.028$ & $18.36 \pm 0.03$ & $18.38 \pm 0.03$ & $18.41 \pm 0.08$ & $18.29 \pm 0.16$ & 1 \\
\hline $090449.79+093143.1$ & - & $\mathrm{DA}+\mathrm{M}$ & 18.394 & $9263 \pm 68$ & $8.85 \pm 0.101$ & $17.08 \pm 0.02$ & $16.56 \pm 0.01$ & $16.04 \pm 0.01$ & $15.76 \pm 0.02$ & 3 \\
\hline $090505.39+054811.7$ & - & $\mathrm{DA}+\mathrm{M}$ & 17.548 & $6919 \pm 74$ & $7.54 \pm 0.15$ & $16.00 \pm 0.01$ & $15.46 \pm 0.00$ & $14.86 \pm 0.01$ & $14.59 \pm 0.01$ & 3 \\
\hline $090516.97+031604.9$ & - & DA & 17.885 & $18414 \pm 114$ & $7.81 \pm 0.022$ & - & - & $17.99 \pm 0.08$ & $18.17 \pm 0.16$ & 1 \\
\hline $090522.94+071519.2$ & - & DA & 18.702 & $8895 \pm 55$ & $8.41 \pm 0.091$ & $18.38 \pm 0.03$ & $18.26 \pm 0.04$ & $18.32 \pm 0.13$ & $18.21 \pm 0.15$ & 1 \\
\hline $090559.60+084324.7$ & - & DA & 18.204 & $10116 \pm 35$ & $8.24 \pm 0.038$ & $17.99 \pm 0.02$ & $17.90 \pm 0.03$ & $17.81 \pm 0.05$ & $17.97 \pm 0.13$ & 1 \\
\hline $090610.67+012223.3$ & $0903+015$ & DA & 19.057 & $7480 \pm 87$ & $8.84 \pm 0.162$ & $18.71 \pm 0.05$ & $18.51 \pm 0.09$ & $18.43 \pm 0.09$ & $18.49 \pm 0.22$ & 2 \\
\hline $090618.44+022311.6$ & $0903+025$ & DA & 18.182 & $7007 \pm 37$ & $8.96 \pm 0.049$ & $17.77 \pm 0.03$ & $17.66 \pm 0.03$ & $17.53 \pm 0.06$ & $17.47 \pm 0.09$ & 2 \\
\hline $090624.25-002428.1$ & - & DA & 18.088 & $11520 \pm 87$ & $8.0 \pm 0.057$ & $17.98 \pm 0.03$ & $17.87 \pm 0.04$ & $17.89 \pm 0.07$ & $17.84 \pm 0.13$ & 2 \\
\hline $090638.58+070059.7$ & - & DA & 15.829 & $17433 \pm 66$ & $7.88 \pm 0.013$ & $15.78 \pm 0.01$ & $15.81 \pm 0.01$ & $15.83 \pm 0.01$ & $15.98 \pm 0.02$ & 1 \\
\hline $090713.40+104248.0$ & - & DA & 17.559 & $7966 \pm 42$ & $8.57 \pm 0.087$ & $17.11 \pm 0.02$ & $16.99 \pm 0.02$ & $16.81 \pm 0.04$ & $16.80 \pm 0.04$ & 1 \\
\hline $090740.57+055348.8$ & - & DA & 18.460 & $9256 \pm 53$ & $8.46 \pm 0.067$ & $18.23 \pm 0.04$ & $18.14 \pm 0.05$ & $18.21 \pm 0.12$ & $18.32 \pm 0.18$ & 1 \\
\hline $090757.30+084524.8$ & - & DA & 18.665 & $16364 \pm 165$ & $7.84 \pm 0.036$ & $18.44 \pm 0.03$ & $18.42 \pm 0.03$ & $18.42 \pm 0.07$ & $18.84 \pm 0.26$ & 1 \\
\hline $090759.59+053649.7$ & - & DA & 18.866 & $19474 \pm 293$ & $7.82 \pm 0.049$ & $18.79 \pm 0.05$ & $18.66 \pm 0.08$ & $18.62 \pm 0.16$ & $18.40 \pm 0.19$ & 1 \\
\hline $090812.04+060421.2$ & - & $\mathrm{DA}+\mathrm{M}$ & 17.073 & $17067 \pm 150$ & $7.05 \pm 0.035$ & $15.92 \pm 0.01$ & $15.40 \pm 0.00$ & $15.07 \pm 0.01$ & $14.68 \pm 0.01$ & 3 \\
\hline $090844.84+072707.6$ & - & $\mathrm{DA}+\mathrm{M}$ & 19.851 & $12750 \pm 242$ & $8.93 \pm 0.086$ & $18.80 \pm 0.05$ & $18.58 \pm 0.05$ & $18.05 \pm 0.09$ & $17.85 \pm 0.11$ & 1 \\
\hline $090850.58+070111.5$ & - & DA & 18.967 & $8049 \pm 104$ & $8.7 \pm 0.231$ & $18.63 \pm 0.04$ & $18.40 \pm 0.05$ & $18.29 \pm 0.12$ & $18.12 \pm 0.15$ & 1 \\
\hline $091047.34+025818.2$ & - & DA & 18.939 & $9372 \pm 73$ & $8.27 \pm 0.124$ & - & - & $18.60 \pm 0.15$ & $18.55 \pm 0.23$ & 1 \\
\hline $091102.43+092340.1$ & - & DA & 19.081 & $11462 \pm 169$ & $8.77 \pm 0.112$ & $18.87 \pm 0.05$ & $18.97 \pm 0.06$ & $18.88 \pm 0.10$ & $19.05 \pm 0.25$ & 1 \\
\hline $091215.43+011958.8$ & $0909+015$ & DA & 18.366 & $55691 \pm 1415$ & $8.03 \pm 0.087$ & $18.45 \pm 0.03$ & $18.65 \pm 0.10$ & $18.36 \pm 0.09$ & $18.47 \pm 0.21$ & 2 \\
\hline $091258.85+091815.6$ & - & DA & 18.342 & $20555 \pm 151$ & $7.79 \pm 0.024$ & $18.37 \pm 0.04$ & $18.34 \pm 0.05$ & $18.27 \pm 0.07$ & $18.56 \pm 0.19$ & 1 \\
\hline
\end{tabular}

Continued on next page 
Table C.1- continued from previous page

\begin{tabular}{|c|c|c|c|c|c|c|c|c|c|c|}
\hline SDSS J & WD & Spec. Type & $i^{\prime}$ & $T_{\text {eff }}(\mathrm{K})$ & $\log g$ & $\bar{Y}$ & $\mathrm{~J}$ & $\mathrm{H}$ & $\bar{K}$ & Ref. \\
\hline $091304.44+011418.6$ & $0910+014$ & $\mathrm{DA}+\mathrm{M}$ & 18.063 & $6300 \pm 1$ & $8.17 \pm 0.326$ & $16.91 \pm 0.01$ & $16.45 \pm 0.01$ & $15.88 \pm 0.01$ & $15.66 \pm 0.02$ & 2 \\
\hline $091341.04+011240.2$ & $0911+014$ & DA & 18.076 & $7935 \pm 41$ & $8.98 \pm 0.031$ & $17.64 \pm 0.02$ & $17.48 \pm 0.03$ & $17.33 \pm 0.04$ & $17.33 \pm 0.08$ & 2 \\
\hline $091437.35+054453.2$ & - & DAH & 17.887 & $26766 \pm 199$ & $9.0 \pm 0.0020$ & $17.86 \pm 0.03$ & $17.88 \pm 0.04$ & $17.94 \pm 0.07$ & $17.89 \pm 0.13$ & 1 \\
\hline $091527.98+094734.2$ & - & DA & 17.757 & $14763 \pm 134$ & $7.54 \pm 0.03$ & $17.62 \pm 0.02$ & $17.68 \pm 0.03$ & $17.74 \pm 0.07$ & $17.78 \pm 0.12$ & 1 \\
\hline $091536.97+094335.1$ & - & DA & 17.581 & $12020 \pm 118$ & $8.16 \pm 0.042$ & $17.42 \pm 0.02$ & $17.41 \pm 0.03$ & $17.36 \pm 0.05$ & $17.48 \pm 0.09$ & 1 \\
\hline $091540.27+102112.0$ & - & $\mathrm{DA}+\mathrm{M}$ & 18.638 & $7023 \pm 70$ & $7.93 \pm 0.138$ & $17.47 \pm 0.02$ & $16.96 \pm 0.01$ & $16.39 \pm 0.02$ & $16.21 \pm 0.03$ & 1 \\
\hline $091601.48-003129.8$ & - & $\mathrm{DA}+\mathrm{M}$ & 17.078 & $10145 \pm 31$ & $8.55 \pm 0.032$ & $15.65 \pm 0.01$ & $15.09 \pm 0.00$ & $14.55 \pm 0.00$ & $14.20 \pm 0.01$ & 2 \\
\hline $091841.84+061702.0$ & $0916+064$ & DA & 16.393 & $45446 \pm 492$ & $7.57 \pm 0.043$ & - & $16.53 \pm 0.01$ & $16.64 \pm 0.02$ & $16.76 \pm 0.04$ & 1 \\
\hline $091924.19+083710.8$ & - & DA & 17.467 & $18806 \pm 91$ & $7.93 \pm 0.016$ & $17.40 \pm 0.02$ & $17.44 \pm 0.02$ & $17.59 \pm 0.05$ & $17.69 \pm 0.13$ & 1 \\
\hline $091927.44+085133.2$ & - & $\mathrm{DA}+\mathrm{M}$ & 18.681 & $8399 \pm 50$ & $8.99 \pm 0.024$ & $17.35 \pm 0.02$ & $16.85 \pm 0.01$ & $16.29 \pm 0.02$ & $16.05 \pm 0.02$ & 3 \\
\hline $092010.55+045721.1$ & - & DA & 17.527 & $70195 \pm 1955$ & $7.58 \pm 0.09$ & - & - & $17.93 \pm 0.11$ & $18.29 \pm 0.20$ & 1 \\
\hline 092017.96-001246.4 & - & $\mathrm{DA}+\mathrm{M}$ & 19.625 & $8028 \pm 137$ & $8.77 \pm 0.166$ & $18.25 \pm 0.03$ & $17.73 \pm 0.03$ & $17.11 \pm 0.03$ & $16.87 \pm 0.05$ & 2 \\
\hline $092048.04+105734.4$ & - & $\mathrm{DA}+\mathrm{M}$ & 17.976 & $66932 \pm 1956$ & $6.99 \pm 0.103$ & $17.31 \pm 0.02$ & $17.00 \pm 0.02$ & $16.60 \pm 0.02$ & $16.39 \pm 0.02$ & 1 \\
\hline $092055.08+085459.9$ & - & DA & 18.876 & $8768 \pm 46$ & $8.99 \pm 0.016$ & $18.48 \pm 0.05$ & $18.46 \pm 0.04$ & $18.30 \pm 0.11$ & $18.53 \pm 0.20$ & 1 \\
\hline $092100.49+043216.5$ & - & $\mathrm{DA}+\mathrm{M}$ & 19.140 & $8841 \pm 91$ & $8.61 \pm 0.125$ & - & - & $16.99 \pm 0.05$ & $16.70 \pm 0.05$ & 1 \\
\hline $092157.64+101351.7$ & - & $\mathrm{DA}+\mathrm{M}$ & 18.745 & $6300 \pm 1$ & $8.81 \pm 0.128$ & $16.90 \pm 0.01$ & $16.34 \pm 0.01$ & $15.82 \pm 0.01$ & $15.52 \pm 0.02$ & 3 \\
\hline $092207.11+014637.1$ & $0919+019$ & DA & 18.606 & $10452 \pm 48$ & $8.28 \pm 0.048$ & $18.33 \pm 0.03$ & $18.26 \pm 0.06$ & $18.49 \pm 0.11$ & $18.01 \pm 0.16$ & 2 \\
\hline $092208.61+053120.3$ & - & DA & 18.112 & $13263 \pm 255$ & $7.9 \pm 0.045$ & - & - & $18.17 \pm 0.14$ & $18.29 \pm 0.21$ & 1 \\
\hline $092308.43+054340.5$ & - & DA & 19.000 & $7298 \pm 81$ & $8.83 \pm 0.155$ & - & - & $17.88 \pm 0.09$ & $17.95 \pm 0.13$ & 1 \\
\hline $092329.81+012020.0$ & $0920+015$ & DA & 18.684 & $11150 \pm 69$ & $8.74 \pm 0.064$ & $18.51 \pm 0.04$ & $18.48 \pm 0.08$ & $18.34 \pm 0.09$ & $18.44 \pm 0.21$ & 2 \\
\hline $092402.32+014715.0$ & $0921+020$ & $\mathrm{DA}+\mathrm{M}$ & 20.260 & $10586 \pm 245$ & $7.79 \pm 0.245$ & $18.86 \pm 0.05$ & $18.34 \pm 0.09$ & $17.75 \pm 0.06$ & $17.66 \pm 0.11$ & 2 \\
\hline $092406.27+012411.4$ & $0921+016$ & $\mathrm{DA}+\mathrm{M}$ & 18.190 & $66579 \pm 2551$ & $6.74 \pm 0.141$ & $17.33 \pm 0.02$ & $16.99 \pm 0.02$ & $16.48 \pm 0.02$ & $16.28 \pm 0.03$ & 2 \\
\hline $092429.06+010448.3$ & $0921+012$ & $\mathrm{DA}+\mathrm{M}$ & 19.565 & $7287 \pm 112$ & $8.19 \pm 0.26$ & $18.27 \pm 0.03$ & $17.66 \pm 0.02$ & $17.22 \pm 0.04$ & $17.10 \pm 0.07$ & 2 \\
\hline 092451.63-001736.4 & - & $\mathrm{DA}+\mathrm{M}$ & 17.420 & $6300 \pm 2$ & $6.85 \pm 0.123$ & $16.06 \pm 0.01$ & $15.54 \pm 0.01$ & $15.02 \pm 0.01$ & $14.71 \pm 0.01$ & 2 \\
\hline $092452.40+002448.9$ & $0922+006$ & $\mathrm{DA}+\mathrm{M}$ & 17.868 & $9702 \pm 37$ & $8.49 \pm 0.034$ & $16.61 \pm 0.01$ & $16.07 \pm 0.01$ & $15.57 \pm 0.01$ & $15.27 \pm 0.01$ & 2 \\
\hline $092511.60+050932.4$ & - & DA & 15.503 & $10883 \pm 26$ & $8.41 \pm 0.015$ & - & - & $15.26 \pm 0.01$ & $15.33 \pm 0.01$ & 1 \\
\hline $092528.22+044952.4$ & - & DA & 18.282 & $10309 \pm 51$ & $8.37 \pm 0.051$ & - & - & $17.91 \pm 0.11$ & $17.99 \pm 0.16$ & 1 \\
\hline $092543.01+055038.5$ & - & DA & 18.193 & $8302 \pm 37$ & $8.45 \pm 0.059$ & - & - & $17.57 \pm 0.07$ & $17.48 \pm 0.08$ & 1 \\
\hline $092547.59+042248.1$ & - & DA & 18.383 & $10221 \pm 48$ & $8.14 \pm 0.056$ & - & - & $18.24 \pm 0.13$ & $18.27 \pm 0.19$ & 1 \\
\hline $092558.33+055909.1$ & - & DA & 18.506 & $10980 \pm 74$ & $8.31 \pm 0.05$ & - & - & $18.28 \pm 0.13$ & $18.10 \pm 0.14$ & 1 \\
\hline $092648.84+102828.8$ & - & DA & 20.492 & $13214 \pm 935$ & $8.11 \pm 0.298$ & $19.57 \pm 0.12$ & $19.19 \pm 0.16$ & $18.74 \pm 0.19$ & $18.29 \pm 0.19$ & 1 \\
\hline $092723.96+002847.5$ & $0924+006$ & DA & 18.733 & $8355 \pm 60$ & $8.88 \pm 0.101$ & $18.39 \pm 0.04$ & $18.21 \pm 0.04$ & $18.00 \pm 0.09$ & $18.08 \pm 0.17$ & 2 \\
\hline
\end{tabular}
Continued on next page 
Table C.1- continued from previous page

\begin{tabular}{|c|c|c|c|c|c|c|c|c|c|c|}
\hline SDSS J & WD & Spec. Type & $i^{\prime}$ & $T_{\text {eff }}(\mathrm{K})$ & $\log g$ & $\bar{Y}$ & $\mathrm{~J}$ & $\mathrm{H}$ & $\bar{K}$ & Ref. \\
\hline $092801.37+025428.3$ & - & $\mathrm{DA}$ & 18.777 & $8635 \pm 58$ & $8.06 \pm 0.118$ & $18.37 \pm 0.04$ & $18.36 \pm 0.06$ & $18.07 \pm 0.10$ & $18.32 \pm 0.21$ & 1 \\
\hline 092834.58-005121.6 & - & DA & 18.542 & $7962 \pm 58$ & $8.82 \pm 0.136$ & $18.06 \pm 0.03$ & $17.94 \pm 0.04$ & $17.89 \pm 0.10$ & $17.79 \pm 0.16$ & 2 \\
\hline $092936.53+081546.4$ & - & DA & 17.049 & $12302 \pm 87$ & $8.05 \pm 0.029$ & $16.88 \pm 0.01$ & $16.91 \pm 0.01$ & $16.91 \pm 0.02$ & $16.98 \pm 0.06$ & 1 \\
\hline $093229.84+082637.3$ & - & DA & 17.175 & $14737 \pm 121$ & $7.91 \pm 0.022$ & $17.08 \pm 0.01$ & $17.10 \pm 0.02$ & $17.14 \pm 0.03$ & $17.28 \pm 0.09$ & 1 \\
\hline $093345.50+062133.1$ & - & DA & 18.244 & $20826 \pm 215$ & $7.85 \pm 0.034$ & $18.25 \pm 0.03$ & $18.20 \pm 0.03$ & $18.36 \pm 0.07$ & $18.64 \pm 0.19$ & 1 \\
\hline $093356.39+102215.6$ & - & DA & 18.721 & $9265 \pm 77$ & $8.87 \pm 0.112$ & $18.31 \pm 0.03$ & $18.20 \pm 0.04$ & $18.17 \pm 0.09$ & $18.26 \pm 0.18$ & 1 \\
\hline $093420.06+051209.2$ & - & $\mathrm{DA}+\mathrm{M}$ & 18.439 & $10317 \pm 175$ & $7.96 \pm 0.476$ & - & - & $16.48 \pm 0.03$ & $16.37 \pm 0.04$ & 1 \\
\hline $093426.60+053753.6$ & - & $\mathrm{DA}+\mathrm{M}$ & 19.646 & $6656 \pm 131$ & $7.97 \pm 0.446$ & - & - & $17.29 \pm 0.05$ & $17.01 \pm 0.06$ & 1 \\
\hline $093432.48+031348.8$ & - & $\mathrm{DA}+\mathrm{M}$ & 18.271 & $32791 \pm 256$ & $7.58 \pm 0.047$ & - & - & $16.34 \pm 0.03$ & $16.10 \pm 0.03$ & 3 \\
\hline $093432.66+065848.6$ & - & DA & 17.820 & $48696 \pm 941$ & $7.59 \pm 0.094$ & $17.80 \pm 0.02$ & $17.77 \pm 0.02$ & $17.85 \pm 0.04$ & $17.86 \pm 0.11$ & 1 \\
\hline $093610.81+074702.1$ & - & DA & 17.461 & $8682 \pm 25$ & $8.44 \pm 0.037$ & $17.13 \pm 0.01$ & $17.00 \pm 0.01$ & $16.93 \pm 0.03$ & $16.89 \pm 0.05$ & 1 \\
\hline $093704.24+064627.5$ & - & DA & 18.503 & $7713 \pm 76$ & $8.36 \pm 0.233$ & $18.10 \pm 0.03$ & $17.95 \pm 0.03$ & $17.81 \pm 0.05$ & $17.78 \pm 0.10$ & 1 \\
\hline $093726.44+063000.8$ & - & $\mathrm{DA}+\mathrm{M}$ & 18.381 & $9461 \pm 94$ & $7.49 \pm 0.135$ & $16.97 \pm 0.01$ & $16.44 \pm 0.01$ & $15.93 \pm 0.01$ & $15.64 \pm 0.01$ & 3 \\
\hline $093743.09+035759.0$ & - & DA & 18.817 & $7926 \pm 87$ & $8.56 \pm 0.201$ & - & - & $18.22 \pm 0.14$ & $18.71 \pm 0.31$ & 1 \\
\hline $093857.71+015321.0$ & $0936+021$ & DA & 19.031 & $8077 \pm 92$ & $8.15 \pm 0.198$ & $18.73 \pm 0.04$ & $18.53 \pm 0.05$ & $18.30 \pm 0.15$ & $18.38 \pm 0.22$ & 2 \\
\hline $093904.03+051114.8$ & - & $\mathrm{DA}+\mathrm{M}$ & 18.984 & $8952 \pm 135$ & $7.67 \pm 0.227$ & - & - & $16.61 \pm 0.03$ & $16.29 \pm 0.03$ & 3 \\
\hline $093911.67+083917.3$ & - & DA & 17.810 & $15427 \pm 127$ & $7.84 \pm 0.025$ & $17.73 \pm 0.02$ & $17.73 \pm 0.04$ & $17.75 \pm 0.07$ & $17.90 \pm 0.13$ & 1 \\
\hline $093958.46+102136.0$ & - & DA & 17.822 & $8567 \pm 26$ & $8.49 \pm 0.039$ & $17.47 \pm 0.02$ & $17.35 \pm 0.02$ & $17.23 \pm 0.04$ & $17.19 \pm 0.06$ & 1 \\
\hline $093958.65+011638.2$ & $0937+015$ & DA & 17.030 & $20000 \pm 127$ & $8.39 \pm 0.018$ & $17.04 \pm 0.01$ & $17.06 \pm 0.02$ & $17.11 \pm 0.05$ & $17.19 \pm 0.08$ & 2 \\
\hline $094058.98+000239.8$ & $0938+002$ & $\mathrm{DA}+\mathrm{M}$ & 20.083 & $6312 \pm 18$ & $7.34 \pm 0.662$ & $18.63 \pm 0.05$ & $18.18 \pm 0.06$ & $17.66 \pm 0.06$ & $17.28 \pm 0.09$ & 2 \\
\hline $094101.87+062221.2$ & - & $\mathrm{DA}+\mathrm{M}$ & 18.103 & $6300 \pm 2$ & $7.47 \pm 0.299$ & $16.61 \pm 0.01$ & $16.09 \pm 0.01$ & $15.59 \pm 0.01$ & $15.38 \pm 0.01$ & 3 \\
\hline $094105.14+033711.0$ & - & DA & 18.724 & $18441 \pm 223$ & $7.83 \pm 0.047$ & - & - & $18.91 \pm 0.22$ & $18.61 \pm 0.23$ & 1 \\
\hline $094122.16+085644.0$ & - & DA & 17.874 & $8509 \pm 33$ & $8.48 \pm 0.06$ & $17.54 \pm 0.02$ & $17.46 \pm 0.02$ & $17.32 \pm 0.04$ & $17.35 \pm 0.07$ & 1 \\
\hline $094251.09+034748.1$ & - & DA & 18.715 & $8265 \pm 54$ & $8.95 \pm 0.062$ & - & - & $18.32 \pm 0.14$ & $17.97 \pm 0.15$ & 1 \\
\hline $094459.43+004306.5$ & $0942+009$ & DA & 17.653 & $15899 \pm 70$ & $7.96 \pm 0.015$ & - & $17.53 \pm 0.02$ & $17.54 \pm 0.06$ & $17.62 \pm 0.11$ & 2 \\
\hline $094534.35+015439.5$ & $0942+021$ & DA & 17.171 & $7711 \pm 28$ & $8.99 \pm 0.012$ & $16.72 \pm 0.01$ & $16.56 \pm 0.01$ & $16.36 \pm 0.03$ & $16.39 \pm 0.04$ & 2 \\
\hline $094640.35+011319.8$ & $0944+014$ & DA & 17.805 & $20056 \pm 160$ & $7.86 \pm 0.025$ & $17.82 \pm 0.02$ & $17.86 \pm 0.03$ & $17.73 \pm 0.10$ & $18.04 \pm 0.16$ & 2 \\
\hline $094716.51+024150.5$ & $0944+029$ & DA & 18.545 & $8137 \pm 46$ & $8.65 \pm 0.095$ & $18.22 \pm 0.04$ & $17.94 \pm 0.06$ & $17.95 \pm 0.09$ & $17.98 \pm 0.14$ & 2 \\
\hline $094720.94+111734.7$ & - & $\mathrm{DA}+\mathrm{M}$ & 18.991 & $74118 \pm 5450$ & $7.75 \pm 0.312$ & $18.35 \pm 0.05$ & $18.03 \pm 0.06$ & $17.78 \pm 0.11$ & $17.50 \pm 0.15$ & 3 \\
\hline $094741.08+101626.5$ & - & DA & 18.172 & $9349 \pm 43$ & $8.23 \pm 0.063$ & $17.92 \pm 0.02$ & $17.85 \pm 0.03$ & $17.80 \pm 0.06$ & $17.75 \pm 0.10$ & 1 \\
\hline 094804.29-000738.1 & - & DA & 18.459 & $10131 \pm 55$ & $8.41 \pm 0.071$ & $18.20 \pm 0.03$ & $18.25 \pm 0.06$ & $17.99 \pm 0.08$ & $18.42 \pm 0.25$ & 2 \\
\hline $094825.55+022735.5$ & $0945+026.1$ & DA & 18.827 & $8424 \pm 46$ & $8.48 \pm 0.08$ & $18.56 \pm 0.04$ & $18.42 \pm 0.08$ & $18.35 \pm 0.11$ & $18.33 \pm 0.20$ & 2 \\
\hline
\end{tabular}

Continued on next page 
Table C.1- continued from previous page

\begin{tabular}{|c|c|c|c|c|c|c|c|c|c|c|}
\hline SDSS J & WD & Spec. Type & $i^{\prime}$ & $T_{\text {eff }}(\mathrm{K})$ & $\log g$ & $\bar{Y}$ & $\mathrm{~J}$ & $\mathrm{H}$ & $\bar{K}$ & Ref. \\
\hline $094844.83+003516.7$ & $0946+008$ & $\overline{\mathrm{DA}}$ & 18.062 & $12644 \pm 168$ & $8.05 \pm 0.053$ & $18.00 \pm 0.03$ & $17.96 \pm 0.03$ & $17.96 \pm 0.07$ & $18.02 \pm 0.15$ & 2 \\
\hline $094900.27+014746.4$ & $0946+020$ & $\mathrm{DA}+\mathrm{M}$ & 18.916 & $37980 \pm 515$ & $7.61 \pm 0.077$ & $18.06 \pm 0.03$ & $17.60 \pm 0.03$ & $17.12 \pm 0.05$ & $16.89 \pm 0.06$ & 2 \\
\hline 094901.28-001909.5 & - & DA & 16.809 & $10707 \pm 30$ & $8.15 \pm 0.028$ & $16.64 \pm 0.01$ & $16.59 \pm 0.01$ & $16.63 \pm 0.03$ & $16.63 \pm 0.04$ & 2 \\
\hline $094904.72+013745.1$ & $0946+018$ & DA & 16.877 & $18195 \pm 72$ & $7.78 \pm 0.013$ & $16.73 \pm 0.01$ & $16.72 \pm 0.01$ & $16.68 \pm 0.03$ & $16.83 \pm 0.05$ & 2 \\
\hline $094929.08+101918.8$ & - & DA & 17.829 & $11751 \pm 107$ & $8.14 \pm 0.045$ & $17.67 \pm 0.02$ & $17.66 \pm 0.03$ & $17.66 \pm 0.06$ & $17.71 \pm 0.10$ & 1 \\
\hline $094940.36+032425.4$ & - & DA & 17.642 & $51324 \pm 679$ & $7.7 \pm 0.053$ & - & - & $17.69 \pm 0.08$ & $17.70 \pm 0.11$ & 1 \\
\hline $094952.73+012603.4$ & $0947+016$ & $\mathrm{DA}+\mathrm{M}$ & 19.565 & $8861 \pm 120$ & $8.54 \pm 0.147$ & $18.16 \pm 0.03$ & $17.69 \pm 0.03$ & $17.04 \pm 0.05$ & $16.91 \pm 0.06$ & 2 \\
\hline $095014.87+030935.5$ & - & DA & 18.156 & $10997 \pm 58$ & $8.27 \pm 0.052$ & - & - & $17.91 \pm 0.10$ & $18.15 \pm 0.16$ & 1 \\
\hline $095108.73+025507.4$ & $0948+031$ & $\mathrm{DA}+\mathrm{M}$ & 18.359 & $9593 \pm 71$ & $7.89 \pm 0.081$ & $17.03 \pm 0.01$ & $16.50 \pm 0.02$ & $15.97 \pm 0.02$ & $15.70 \pm 0.02$ & 2 \\
\hline $095110.51+100911.8$ & - & DA & 17.888 & $7543 \pm 50$ & $8.88 \pm 0.12$ & $17.38 \pm 0.02$ & $17.21 \pm 0.02$ & $17.01 \pm 0.03$ & $17.02 \pm 0.06$ & 1 \\
\hline $095156.16+080933.1$ & - & DA & 17.288 & $13869 \pm 153$ & $7.85 \pm 0.021$ & $17.15 \pm 0.01$ & $17.17 \pm 0.02$ & $17.22 \pm 0.04$ & $17.21 \pm 0.06$ & 1 \\
\hline $095230.43+114202.2$ & - & DA & 17.212 & $27126 \pm 102$ & $7.96 \pm 0.022$ & - & - & $17.37 \pm 0.04$ & $17.57 \pm 0.11$ & 1 \\
\hline $095245.58+020938.9$ & $0950+023$ & DA & 17.207 & $43114 \pm 443$ & $7.8 \pm 0.039$ & $17.23 \pm 0.02$ & $17.34 \pm 0.04$ & $17.47 \pm 0.06$ & $17.53 \pm 0.10$ & 2 \\
\hline $095329.06+092409.5$ & - & DA & 17.998 & $36103 \pm 259$ & $7.78 \pm 0.049$ & $18.08 \pm 0.03$ & $18.12 \pm 0.04$ & $18.34 \pm 0.10$ & $18.14 \pm 0.17$ & 1 \\
\hline $095723.82+020734.4$ & $0954+023$ & $\mathrm{DA}+\mathrm{M}$ & 18.636 & $9468 \pm 73$ & $7.65 \pm 0.121$ & $16.97 \pm 0.01$ & $16.44 \pm 0.01$ & $15.92 \pm 0.01$ & $15.61 \pm 0.02$ & 2 \\
\hline 095759.16-010707.5 & 0955-008 & DA & 17.617 & $8124 \pm 30$ & $8.54 \pm 0.054$ & $17.25 \pm 0.02$ & $17.13 \pm 0.02$ & $17.01 \pm 0.04$ & $17.13 \pm 0.07$ & 2 \\
\hline 095810.67-010417.7 & 0955-008.1 & DA & 17.138 & $23442 \pm 164$ & $7.88 \pm 0.02$ & $17.11 \pm 0.01$ & $17.17 \pm 0.02$ & $17.22 \pm 0.05$ & $17.35 \pm 0.09$ & 2 \\
\hline $095833.13+013049.2$ & $0955+017$ & DA & 17.073 & $11677 \pm 57$ & $7.99 \pm 0.028$ & $16.93 \pm 0.01$ & $16.95 \pm 0.02$ & $16.95 \pm 0.03$ & $17.00 \pm 0.06$ & 2 \\
\hline $095934.24+025738.1$ & $0956+032$ & DA & 18.107 & $7894 \pm 45$ & $8.53 \pm 0.094$ & - & $17.54 \pm 0.03$ & $17.37 \pm 0.05$ & $17.29 \pm 0.06$ & 2 \\
\hline $095936.19+034949.3$ & - & DA & 18.288 & $7970 \pm 40$ & $8.51 \pm 0.075$ & - & - & $17.60 \pm 0.07$ & $17.72 \pm 0.13$ & 1 \\
\hline $100010.84+084312.2$ & - & $\mathrm{DA}+\mathrm{M}$ & 18.885 & $6309 \pm 16$ & $8.03 \pm 0.586$ & $17.42 \pm 0.01$ & $16.91 \pm 0.01$ & $16.32 \pm 0.02$ & $16.07 \pm 0.02$ & 3 \\
\hline $100014.23+023742.1$ & $0957+028$ & $\mathrm{DA}+\mathrm{M}$ & 19.692 & $8386 \pm 144$ & $8.57 \pm 0.195$ & $18.22 \pm 0.03$ & $17.66 \pm 0.04$ & $17.19 \pm 0.04$ & $16.96 \pm 0.06$ & 2 \\
\hline $100117.49+020956.0$ & $0958+024$ & $\mathrm{DA}+\mathrm{M}$ & 19.689 & $8304 \pm 126$ & $8.31 \pm 0.187$ & $18.84 \pm 0.05$ & $18.40 \pm 0.06$ & $17.98 \pm 0.08$ & $18.00 \pm 0.15$ & 2 \\
\hline $100143.21+104518.2$ & - & DA & 18.858 & $8479 \pm 61$ & $8.32 \pm 0.117$ & - & $18.47 \pm 0.07$ & $18.42 \pm 0.15$ & $18.39 \pm 0.17$ & 1 \\
\hline $100214.59+010826.3$ & - & DA & 18.254 & $13017 \pm 249$ & $7.98 \pm 0.053$ & $18.18 \pm 0.03$ & $18.14 \pm 0.04$ & $18.10 \pm 0.09$ & $17.94 \pm 0.18$ & 1 \\
\hline $100259.88+093950.0$ & - & DA & 19.585 & $22331 \pm 435$ & $7.87 \pm 0.069$ & $19.30 \pm 0.08$ & $19.19 \pm 0.13$ & $18.55 \pm 0.18$ & $18.55 \pm 0.22$ & 1 \\
\hline $100310.05+041108.2$ & - & $\mathrm{DA}+\mathrm{M}$ & 18.896 & $6300 \pm 2$ & $8.39 \pm 0.433$ & - & - & $16.41 \pm 0.02$ & $16.09 \pm 0.03$ & 3 \\
\hline $100316.35-002336.9$ & - & DA & 16.556 & $19788 \pm 109$ & $7.87 \pm 0.018$ & $16.51 \pm 0.01$ & $16.59 \pm 0.02$ & $16.60 \pm 0.03$ & $16.65 \pm 0.05$ & 2 \\
\hline $100334.41+103756.3$ & - & DA & 18.769 & $14094 \pm 457$ & $7.94 \pm 0.058$ & - & $18.65 \pm 0.08$ & $18.48 \pm 0.15$ & $18.37 \pm 0.16$ & 1 \\
\hline $100417.44+105246.9$ & - & DA & 19.058 & $7503 \pm 69$ & $8.94 \pm 0.073$ & - & $18.52 \pm 0.08$ & $18.44 \pm 0.12$ & $18.32 \pm 0.15$ & 1 \\
\hline $100419.35+044358.4$ & - & DA & 18.078 & $9692 \pm 37$ & $8.38 \pm 0.047$ & - & $17.73 \pm 0.03$ & $17.73 \pm 0.07$ & $17.80 \pm 0.16$ & 1 \\
\hline $100547.72+030828.2$ & - & DA & 17.654 & $8380 \pm 31$ & $8.47 \pm 0.054$ & - & $17.19 \pm 0.02$ & $17.05 \pm 0.05$ & $16.98 \pm 0.05$ & 1 \\
\hline
\end{tabular}

Continued on next page 
Table C.1- continued from previous page

\begin{tabular}{|c|c|c|c|c|c|c|c|c|c|c|}
\hline SDSS J & WD & Spec. Type & $i^{\prime}$ & $T_{\text {eff }}(\mathrm{K})$ & $\log g$ & $\bar{Y}$ & $\mathrm{~J}$ & $\mathrm{H}$ & $\bar{K}$ & Ref. \\
\hline $100609.18+004417.0$ & $1003+009$ & $\mathrm{DA}+\mathrm{M}$ & 17.368 & $7286 \pm 36$ & $7.78 \pm 0.082$ & $15.69 \pm 0.01$ & $14.99 \pm 0.00$ & $14.48 \pm 0.00$ & $14.10 \pm 0.01$ & 2 \\
\hline $100623.07+071212.5$ & - & $\mathrm{DA}$ & 16.150 & $9624 \pm 20$ & $8.27 \pm 0.022$ & $15.88 \pm 0.01$ & $15.84 \pm 0.01$ & $15.75 \pm 0.01$ & $15.77 \pm 0.02$ & 1 \\
\hline $100627.97+083038.7$ & - & DA & 18.750 & $7639 \pm 81$ & $8.27 \pm 0.214$ & - & $18.10 \pm 0.04$ & $17.96 \pm 0.09$ & $17.81 \pm 0.10$ & 1 \\
\hline $100658.64+121133.9$ & - & $\mathrm{DA}+\mathrm{M}$ & 18.644 & $6313 \pm 18$ & $7.2 \pm 0.265$ & $17.54 \pm 0.02$ & $17.08 \pm 0.02$ & $16.52 \pm 0.02$ & $16.37 \pm 0.04$ & 3 \\
\hline $100826.97+031653.0$ & - & DA & 18.266 & $18827 \pm 197$ & $7.57 \pm 0.041$ & - & $18.14 \pm 0.05$ & $18.11 \pm 0.12$ & $18.32 \pm 0.16$ & 1 \\
\hline $100955.37+000944.0$ & $1007+004$ & DA & 17.891 & $10559 \pm 42$ & $8.23 \pm 0.035$ & $17.63 \pm 0.03$ & $17.63 \pm 0.04$ & $17.63 \pm 0.07$ & $17.57 \pm 0.10$ & 2 \\
\hline $101033.21+100748.7$ & - & DA & 18.349 & $13465 \pm 187$ & $7.96 \pm 0.037$ & $18.24 \pm 0.03$ & $18.26 \pm 0.05$ & $18.08 \pm 0.12$ & $18.36 \pm 0.17$ & 1 \\
\hline $101249.37+044135.9$ & - & $\mathrm{DA}+\mathrm{M}$ & 19.979 & $53053 \pm 3351$ & $7.31 \pm 0.293$ & - & - & $18.28 \pm 0.15$ & $18.28 \pm 0.18$ & 3 \\
\hline $101312.71+040514.1$ & $1010+043$ & DA & 17.147 & $29049 \pm 151$ & $7.97 \pm 0.019$ & - & - & $17.17 \pm 0.06$ & $17.51 \pm 0.10$ & 1 \\
\hline $101323.90+043946.1$ & - & $\mathrm{DA}+\mathrm{M}$ & 18.584 & $9689 \pm 52$ & $8.03 \pm 0.072$ & - & - & $16.84 \pm 0.05$ & $16.54 \pm 0.05$ & 3 \\
\hline $101328.16+061207.3$ & $1010+064$ & DA & 17.100 & $50050 \pm 646$ & $7.98 \pm 0.049$ & - & - & $17.48 \pm 0.08$ & $17.52 \pm 0.14$ & 1 \\
\hline $101414.45+040137.4$ & - & DA & 16.684 & $7847 \pm 18$ & $8.51 \pm 0.032$ & - & - & $15.95 \pm 0.02$ & $15.94 \pm 0.03$ & 1 \\
\hline $101502.55+080636.1$ & - & DA & 15.858 & $7441 \pm 18$ & $9.0 \pm 0.0060$ & $15.32 \pm 0.01$ & $15.16 \pm 0.01$ & $14.94 \pm 0.01$ & $14.92 \pm 0.01$ & 1 \\
\hline $101529.62+090703.7$ & - & DAH & 18.391 & $7038 \pm 48$ & $8.98 \pm 0.027$ & $18.01 \pm 0.03$ & $17.86 \pm 0.04$ & $17.69 \pm 0.04$ & $17.66 \pm 0.11$ & 1 \\
\hline $101531.60+123639.3$ & - & DA & 18.848 & $8583 \pm 69$ & $8.53 \pm 0.162$ & - & $18.38 \pm 0.04$ & $18.25 \pm 0.07$ & $18.25 \pm 0.16$ & 1 \\
\hline $101532.10+042507.5$ & - & DA & 18.465 & $35073 \pm 195$ & $7.38 \pm 0.04$ & - & - & $18.42 \pm 0.17$ & $18.17 \pm 0.19$ & 1 \\
\hline $101548.02+030648.2$ & $1013+033$ & DA & 15.985 & $11584 \pm 32$ & $8.14 \pm 0.015$ & - & $15.80 \pm 0.01$ & $15.82 \pm 0.01$ & $15.87 \pm 0.02$ & 2 \\
\hline $101612.13+011156.8$ & $1013+014$ & DA & 19.070 & $9880 \pm 64$ & $8.41 \pm 0.094$ & $18.80 \pm 0.07$ & $18.94 \pm 0.10$ & $18.99 \pm 0.21$ & $18.46 \pm 0.23$ & 2 \\
\hline $101642.93+044317.7$ & - & DA & 18.986 & $27039 \pm 276$ & $7.43 \pm 0.049$ & - & - & $17.73 \pm 0.09$ & $17.59 \pm 0.09$ & 1 \\
\hline $101650.40+080749.3$ & - & $\mathrm{DA}+\mathrm{M}$ & 19.423 & $21575 \pm 490$ & $7.59 \pm 0.107$ & $18.48 \pm 0.04$ & $18.06 \pm 0.05$ & $17.56 \pm 0.05$ & $17.26 \pm 0.06$ & 1 \\
\hline $101740.10+033106.9$ & - & DA & 18.443 & $18448 \pm 169$ & $7.42 \pm 0.033$ & - & $18.32 \pm 0.05$ & $18.51 \pm 0.15$ & $18.89 \pm 0.28$ & 1 \\
\hline $101741.69-002934.0$ & - & DA & 16.974 & $14213 \pm 120$ & $7.9 \pm 0.02$ & $16.89 \pm 0.01$ & $16.91 \pm 0.01$ & $16.88 \pm 0.03$ & $16.92 \pm 0.05$ & 2 \\
\hline $101805.04+011123.6$ & $1015+014$ & DAH & 16.542 & $10088 \pm 20$ & $7.2 \pm 0.112$ & $16.39 \pm 0.01$ & $16.33 \pm 0.01$ & $16.34 \pm 0.02$ & $16.32 \pm 0.03$ & 2 \\
\hline $101911.50+000017.2$ & $1016+002$ & DA & 18.486 & $12747 \pm 142$ & $8.3 \pm 0.054$ & $18.30 \pm 0.04$ & $18.31 \pm 0.07$ & $18.59 \pm 0.15$ & $18.38 \pm 0.21$ & 2 \\
\hline $102003.38+000902.6$ & - & DA & 18.625 & $10120 \pm 55$ & $8.38 \pm 0.464$ & $18.33 \pm 0.04$ & $18.31 \pm 0.06$ & $18.38 \pm 0.12$ & $17.94 \pm 0.14$ & 2 \\
\hline $102251.60+013117.3$ & $1020+017$ & DA & 18.487 & $12066 \pm 118$ & $8.12 \pm 0.05$ & - & - & $18.41 \pm 0.18$ & $18.14 \pm 0.20$ & 2 \\
\hline $102546.70+002857.1$ & $1023+007$ & DA & 17.585 & $8339 \pm 32$ & $8.48 \pm 0.052$ & - & - & $16.98 \pm 0.04$ & $16.91 \pm 0.06$ & 2 \\
\hline $102549.71+003906.1$ & $1023+009$ & DA & 16.856 & $38182 \pm 116$ & $7.85 \pm 0.032$ & - & - & $17.11 \pm 0.04$ & $17.26 \pm 0.08$ & 2 \\
\hline $102822.33+034416.5$ & - & DA & 17.449 & $8196 \pm 36$ & $8.55 \pm 0.065$ & $17.08 \pm 0.02$ & $16.97 \pm 0.02$ & $16.80 \pm 0.04$ & $16.94 \pm 0.08$ & 1 \\
\hline $102918.97+021121.9$ & - & DA & 19.282 & $7931 \pm 107$ & $8.74 \pm 0.191$ & - & - & $18.49 \pm 0.17$ & $18.49 \pm 0.25$ & 1 \\
\hline $102936.18+015010.6$ & - & DA & 17.934 & $29251 \pm 170$ & $7.13 \pm 0.037$ & - & - & $18.18 \pm 0.14$ & $18.12 \pm 0.19$ & 1 \\
\hline $103220.19+011227.0$ & - & DA & 19.284 & $8901 \pm 93$ & $7.71 \pm 0.183$ & - & - & $17.76 \pm 0.08$ & $17.15 \pm 0.07$ & 1 \\
\hline
\end{tabular}

Continued on next page 
Table C.1- continued from previous page

\begin{tabular}{|c|c|c|c|c|c|c|c|c|c|c|}
\hline SDSS J & WD & Spec. Type & $i^{\prime}$ & $T_{\text {eff }}(\mathrm{K})$ & $\log g$ & $\bar{Y}$ & $\mathrm{~J}$ & $\mathrm{H}$ & $\bar{K}$ & Ref. \\
\hline $103430.51+022530.8$ & - & $\overline{D A}$ & 18.513 & $7566 \pm 59$ & $8.14 \pm 0.181$ & - & - & $17.63 \pm 0.09$ & $17.52 \pm 0.12$ & 1 \\
\hline $103448.92+005201.4$ & $1032+011$ & DA & 19.158 & $9904 \pm 109$ & $8.13 \pm 0.158$ & - & - & $18.20 \pm 0.11$ & $18.03 \pm 0.14$ & 2 \\
\hline $103500.91+023008.6$ & - & $\mathrm{DA}+\mathrm{M}$ & 18.843 & $72414 \pm 3641$ & $7.21 \pm 0.191$ & - & - & $17.64 \pm 0.08$ & $17.57 \pm 0.11$ & 1 \\
\hline $103540.79+072716.7$ & - & DA & 18.187 & $7802 \pm 41$ & $8.94 \pm 0.066$ & - & - & $17.31 \pm 0.07$ & $17.44 \pm 0.11$ & 1 \\
\hline $103647.97-003237.2$ & - & DA & 18.595 & $9574 \pm 71$ & $8.31 \pm 0.106$ & - & - & $18.04 \pm 0.14$ & $17.85 \pm 0.16$ & 2 \\
\hline $103736.75+013912.2$ & - & DA & 18.813 & $16300 \pm 221$ & $7.73 \pm 0.053$ & - & - & $17.25 \pm 0.06$ & $17.12 \pm 0.07$ & 1 \\
\hline $103837.22+015058.4$ & - & $\mathrm{DA}+\mathrm{M}$ & 17.399 & $35368 \pm 273$ & $7.65 \pm 0.053$ & - & - & $15.42 \pm 0.01$ & $14.94 \pm 0.01$ & 3 \\
\hline $103837.71+020229.2$ & - & DA & 18.161 & $7755 \pm 55$ & $8.83 \pm 0.137$ & - & - & $17.38 \pm 0.06$ & $17.51 \pm 0.10$ & 1 \\
\hline $104057.47+083421.6$ & - & $\mathrm{DA}+\mathrm{M}$ & 17.493 & $8664 \pm 42$ & $6.62 \pm 0.082$ & - & - & $14.89 \pm 0.01$ & $14.63 \pm 0.01$ & 1 \\
\hline $104321.16+030630.4$ & $1040+033$ & DA & 18.431 & $18564 \pm 144$ & $7.95 \pm 0.026$ & - & - & $18.31 \pm 0.12$ & $18.65 \pm 0.24$ & 2 \\
\hline $104411.38+021436.5$ & $1041+025$ & DA & 17.741 & $7761 \pm 37$ & $8.65 \pm 0.087$ & - & - & $16.95 \pm 0.05$ & $17.01 \pm 0.06$ & 2 \\
\hline $104517.78-001833.8$ & - & $\mathrm{DA}+\mathrm{M}$ & 17.964 & $9542 \pm 42$ & $8.09 \pm 0.06$ & $16.47 \pm 0.01$ & $15.90 \pm 0.01$ & $15.37 \pm 0.01$ & $15.07 \pm 0.01$ & 2 \\
\hline $104623.27+024236.5$ & - & DA & 16.475 & $13316 \pm 116$ & $7.88 \pm 0.02$ & - & - & $16.41 \pm 0.03$ & $16.47 \pm 0.04$ & 1 \\
\hline $104933.58+022451.7$ & - & DA & 19.198 & $7590 \pm 96$ & $7.76 \pm 0.324$ & - & - & $17.80 \pm 0.09$ & $17.13 \pm 0.06$ & 1 \\
\hline $104946.45+003635.1$ & $1047+008$ & DA & 17.877 & $22940 \pm 167$ & $8.02 \pm 0.021$ & - & - & $17.99 \pm 0.08$ & $18.09 \pm 0.15$ & 2 \\
\hline $105332.62+020126.2$ & - & DA & 19.062 & $7530 \pm 90$ & $8.14 \pm 0.303$ & - & - & $17.26 \pm 0.05$ & $17.06 \pm 0.07$ & 1 \\
\hline $105612.31-000621.7$ & - & DA & 17.888 & $11016 \pm 51$ & $7.86 \pm 0.033$ & $17.73 \pm 0.04$ & $17.77 \pm 0.05$ & $17.70 \pm 0.08$ & $17.86 \pm 0.13$ & 2 \\
\hline $105709.78+023637.8$ & - & DA & 18.381 & $8403 \pm 52$ & $8.57 \pm 0.09$ & - & - & $17.92 \pm 0.11$ & $17.82 \pm 0.13$ & 1 \\
\hline $105709.81+041130.3$ & - & DA & 17.604 & $7807 \pm 31$ & $8.99 \pm 0.015$ & - & - & $17.06 \pm 0.05$ & $16.96 \pm 0.08$ & 1 \\
\hline $105727.81+002118.6$ & $1054+006$ & DA & 18.899 & $10705 \pm 68$ & $8.42 \pm 0.05$ & $18.61 \pm 0.08$ & - & $18.82 \pm 0.22$ & $18.45 \pm 0.23$ & 2 \\
\hline $105853.59+005608.5$ & $1056+012$ & $\mathrm{DA}+\mathrm{M}$ & 18.234 & $7024 \pm 70$ & $7.17 \pm 0.18$ & - & - & $16.33 \pm 0.02$ & $16.10 \pm 0.03$ & 2 \\
\hline $110326.70+003725.8$ & $1100+008$ & DA & 17.851 & $10537 \pm 48$ & $8.22 \pm 0.047$ & - & - & $17.64 \pm 0.10$ & $17.69 \pm 0.15$ & 2 \\
\hline $110515.31+001626.1$ & $1102+005$ & DA & 15.622 & $12844 \pm 59$ & $8.26 \pm 0.018$ & $15.45 \pm 0.01$ & $15.48 \pm 0.01$ & $15.51 \pm 0.01$ & $15.55 \pm 0.02$ & 2 \\
\hline $110627.66-010514.6$ & $1103-008$ & $\mathrm{DA}+\mathrm{M}$ & 16.783 & $31130 \pm 96$ & $7.37 \pm 0.018$ & - & - & $14.93 \pm 0.01$ & $14.64 \pm 0.01$ & 2 \\
\hline $110844.13+080139.2$ & - & $\mathrm{DA}$ & 16.060 & $7966 \pm 18$ & $8.53 \pm 0.028$ & - & - & $15.37 \pm 0.01$ & $15.36 \pm 0.02$ & 1 \\
\hline $110944.78+044049.5$ & - & DA & 18.702 & $7529 \pm 65$ & $8.78 \pm 0.181$ & - & - & $17.90 \pm 0.08$ & $17.83 \pm 0.17$ & 1 \\
\hline $111047.51+005421.3$ & $1108+011$ & DA & 17.512 & $8968 \pm 26$ & $8.49 \pm 0.041$ & - & - & $17.04 \pm 0.05$ & $17.10 \pm 0.08$ & 2 \\
\hline $111230.13+003002.6$ & $1109+007$ & DA & 18.427 & $9724 \pm 34$ & $8.27 \pm 0.043$ & $18.11 \pm 0.03$ & $18.02 \pm 0.05$ & $18.25 \pm 0.19$ & $18.47 \pm 0.28$ & 2 \\
\hline $111629.75+060014.3$ & - & DA & 18.800 & $9477 \pm 71$ & $7.93 \pm 0.111$ & - & - & $18.05 \pm 0.11$ & $17.86 \pm 0.18$ & 1 \\
\hline $111634.88+022338.1$ & - & $\mathrm{DA}+\mathrm{M}$ & 18.831 & $10599 \pm 96$ & $8.1 \pm 0.082$ & - & - & $16.83 \pm 0.04$ & $16.54 \pm 0.04$ & 3 \\
\hline $111735.11+012940.0$ & - & $\mathrm{DA}+\mathrm{M}$ & 17.203 & $8014 \pm 32$ & $7.62 \pm 0.079$ & $15.52 \pm 0.01$ & $14.88 \pm 0.00$ & $14.40 \pm 0.01$ & $14.04 \pm 0.01$ & 3 \\
\hline $111816.39+040206.7$ & - & DA & 17.713 & $9129 \pm 31$ & $8.29 \pm 0.053$ & - & - & $17.25 \pm 0.05$ & $17.37 \pm 0.10$ & 1 \\
\hline
\end{tabular}

Continued on next page 
Table C.1- continued from previous page

\begin{tabular}{|c|c|c|c|c|c|c|c|c|c|c|}
\hline SDSS J & WD & Spec. Type & $i^{\prime}$ & $T_{\text {eff }}(\mathrm{K})$ & $\log g$ & $\bar{Y}$ & $\mathrm{~J}$ & $\mathrm{H}$ & $\bar{K}$ & Ref. \\
\hline $111946.60+060523.5$ & - & $\mathrm{DA}$ & 18.758 & $9567 \pm 62$ & $8.25 \pm 0.092$ & - & - & $18.44 \pm 0.16$ & $18.04 \pm 0.21$ & 1 \\
\hline $112221.10+035822.4$ & - & DA & 18.306 & $11072 \pm 80$ & $8.06 \pm 0.061$ & - & - & $18.09 \pm 0.10$ & $18.07 \pm 0.17$ & 1 \\
\hline $112307.36+003216.2$ & $1120+008$ & $\mathrm{DA}+\mathrm{M}$ & 18.847 & $9775 \pm 78$ & $7.98 \pm 0.082$ & $17.45 \pm 0.02$ & $16.90 \pm 0.02$ & $16.38 \pm 0.03$ & $16.04 \pm 0.03$ & 2 \\
\hline $112357.72-011027.5$ & $1121-008$ & $\mathrm{DA}+\mathrm{M}$ & 18.044 & $9399 \pm 42$ & $8.6 \pm 0.052$ & $16.83 \pm 0.01$ & $16.35 \pm 0.01$ & $15.85 \pm 0.02$ & $15.58 \pm 0.02$ & 2 \\
\hline $112448.36+024111.7$ & $1122+029$ & DA & 18.458 & $7352 \pm 61$ & $8.81 \pm 0.164$ & - & - & $17.62 \pm 0.08$ & $17.62 \pm 0.11$ & 2 \\
\hline $112542.84+034506.3$ & - & DA & 18.370 & $11600 \pm 123$ & $7.99 \pm 0.07$ & - & - & $18.19 \pm 0.13$ & $18.08 \pm 0.18$ & 1 \\
\hline $112613.51-002109.6$ & - & DA & 18.525 & $17734 \pm 181$ & $7.94 \pm 0.036$ & $18.52 \pm 0.05$ & $18.45 \pm 0.08$ & $18.63 \pm 0.20$ & $18.23 \pm 0.20$ & 2 \\
\hline $112623.87+010856.9$ & $1123+014$ & $\mathrm{DA}+\mathrm{M}$ & 17.386 & $6300 \pm 1$ & $6.99 \pm 0.087$ & - & $15.52 \pm 0.01$ & $14.96 \pm 0.01$ & $14.71 \pm 0.01$ & 2 \\
\hline $112717.06+075257.7$ & - & DA & 18.796 & $8802 \pm 51$ & $8.45 \pm 0.076$ & - & - & $17.89 \pm 0.08$ & $18.30 \pm 0.17$ & 1 \\
\hline $112731.55+062305.1$ & - & DA & 17.124 & $8690 \pm 21$ & $8.41 \pm 0.034$ & - & - & $16.54 \pm 0.03$ & $16.60 \pm 0.05$ & 1 \\
\hline $113109.06+064306.0$ & - & DA & 17.218 & $7921 \pm 29$ & $8.43 \pm 0.052$ & - & - & $16.50 \pm 0.03$ & $16.51 \pm 0.05$ & 1 \\
\hline $113416.09+055227.2$ & - & DA & 19.582 & $24184 \pm 650$ & $7.85 \pm 0.089$ & - & - & $18.10 \pm 0.12$ & $17.94 \pm 0.20$ & 1 \\
\hline $113655.17+040952.6$ & - & $\mathrm{DA}+\mathrm{M}$ & 16.812 & $10496 \pm 44$ & $7.94 \pm 0.046$ & $15.38 \pm 0.00$ & $14.78 \pm 0.00$ & $14.28 \pm 0.00$ & $13.93 \pm 0.01$ & 3 \\
\hline $113725.15-002259.0$ & - & DA & 18.004 & $8553 \pm 33$ & $8.35 \pm 0.065$ & $17.70 \pm 0.02$ & $17.52 \pm 0.03$ & $17.52 \pm 0.08$ & $17.55 \pm 0.10$ & 2 \\
\hline $113738.37-002721.6$ & - & DA & 17.961 & $8739 \pm 30$ & $8.36 \pm 0.055$ & - & $17.42 \pm 0.03$ & $17.44 \pm 0.03$ & $17.49 \pm 0.09$ & 2 \\
\hline $113747.13+041230.7$ & - & DA & 17.197 & $27753 \pm 122$ & $7.84 \pm 0.024$ & - & - & $17.49 \pm 0.08$ & $17.87 \pm 0.20$ & 1 \\
\hline $113800.34-001144.4$ & - & $\mathrm{DA}+\mathrm{M}$ & 18.153 & $7798 \pm 87$ & $7.94 \pm 0.162$ & $16.69 \pm 0.02$ & $16.14 \pm 0.01$ & $15.55 \pm 0.01$ & $15.26 \pm 0.01$ & 2 \\
\hline $113839.51-014902.9$ & $1136-015$ & DAHpec & 17.947 & $10120 \pm 53$ & $8.87 \pm 0.125$ & $17.81 \pm 0.04$ & $17.81 \pm 0.05$ & $17.83 \pm 0.08$ & $17.73 \pm 0.13$ & 1 \\
\hline $114132.98+042028.8$ & - & $\mathrm{DA}+\mathrm{M}$ & 18.545 & $11520 \pm 187$ & $7.53 \pm 0.131$ & - & - & $17.08 \pm 0.05$ & $16.61 \pm 0.07$ & 1 \\
\hline $114209.82+012607.4$ & $1139+017$ & DA & 18.372 & $8415 \pm 45$ & $8.4 \pm 0.1$ & $17.96 \pm 0.03$ & $17.88 \pm 0.04$ & $17.70 \pm 0.07$ & $17.73 \pm 0.13$ & 2 \\
\hline $114312.57+000926.6$ & $1140+004$ & $\mathrm{DA}+\mathrm{M}$ & 17.646 & $9760 \pm 47$ & $8.07 \pm 0.064$ & $16.25 \pm 0.01$ & $15.70 \pm 0.01$ & $15.19 \pm 0.01$ & $14.89 \pm 0.01$ & 2 \\
\hline $114314.73+032239.1$ & $1140+036$ & DA & 18.680 & $8864 \pm 53$ & $8.24 \pm 0.102$ & $18.33 \pm 0.05$ & - & $18.21 \pm 0.12$ & $18.16 \pm 0.20$ & 2 \\
\hline $114756.00+004340.8$ & $1145+010$ & DA & 18.316 & $9962 \pm 47$ & $8.36 \pm 0.064$ & $18.12 \pm 0.04$ & $18.11 \pm 0.05$ & $17.90 \pm 0.08$ & $18.26 \pm 0.17$ & 2 \\
\hline $114832.45+034934.4$ & - & $\mathrm{DA}+\mathrm{M}$ & 17.541 & $20048 \pm 136$ & $7.61 \pm 0.023$ & $15.02 \pm 0.00$ & - & $13.99 \pm 0.00$ & $13.72 \pm 0.00$ & 3 \\
\hline $114913.52-014728.5$ & $1146-015$ & $\mathrm{DA}+\mathrm{M}$ & 18.147 & $17476 \pm 206$ & $7.09 \pm 0.048$ & $15.93 \pm 0.01$ & $15.41 \pm 0.01$ & $14.97 \pm 0.01$ & $14.68 \pm 0.01$ & 2 \\
\hline $115105.83-001135.8$ & - & DA & 18.905 & $7619 \pm 84$ & $8.59 \pm 0.227$ & $18.39 \pm 0.04$ & $18.16 \pm 0.07$ & $17.99 \pm 0.09$ & $18.10 \pm 0.16$ & 2 \\
\hline $115156.94-000725.4$ & - & $\mathrm{DA}+\mathrm{M}$ & 17.815 & $9499 \pm 47$ & $7.57 \pm 0.078$ & $16.47 \pm 0.01$ & $15.97 \pm 0.01$ & $15.51 \pm 0.01$ & $15.13 \pm 0.01$ & 2 \\
\hline $115236.99+002141.8$ & $1150+006$ & $\mathrm{DA}+\mathrm{M}$ & 17.314 & $10044 \pm 24$ & $7.86 \pm 0.033$ & $16.38 \pm 0.01$ & $15.91 \pm 0.01$ & $15.41 \pm 0.01$ & $15.02 \pm 0.01$ & 2 \\
\hline $115707.43+055303.5$ & - & DA & 17.814 & $11053 \pm 53$ & $8.15 \pm 0.039$ & - & - & $17.66 \pm 0.09$ & $17.71 \pm 0.17$ & 1 \\
\hline $115904.91+112716.2$ & - & $\mathrm{DA}+\mathrm{M}$ & 18.976 & $10467 \pm 85$ & $8.45 \pm 0.074$ & - & - & $16.62 \pm 0.03$ & $16.36 \pm 0.05$ & 3 \\
\hline $120039.31+042752.7$ & - & DA & 18.667 & $7286 \pm 62$ & $8.44 \pm 0.194$ & $18.16 \pm 0.04$ & $18.04 \pm 0.06$ & $17.73 \pm 0.08$ & $18.18 \pm 0.21$ & 1 \\
\hline $120125.40+084800.4$ & - & DA & 18.554 & $7105 \pm 55$ & $8.97 \pm 0.046$ & $18.11 \pm 0.04$ & $17.94 \pm 0.06$ & $17.80 \pm 0.06$ & $17.84 \pm 0.13$ & 1 \\
\hline
\end{tabular}

Continued on next page 
Table C.1- continued from previous page

\begin{tabular}{|c|c|c|c|c|c|c|c|c|c|c|}
\hline SDSS J & WD & Spec. Type & $i^{\prime}$ & $T_{\text {eff }}(\mathrm{K})$ & $\log g$ & $\mathrm{Y}$ & $\mathrm{J}$ & $\mathrm{H}$ & $\mathrm{K}$ & Ref. \\
\hline $120558.54-002740.0$ & - & DA & 18.195 & $8623 \pm 52$ & $8.34 \pm 0.125$ & $17.86 \pm 0.03$ & $17.76 \pm 0.04$ & $17.56 \pm 0.08$ & $17.96 \pm 0.19$ & 2 \\
\hline $120604.13-003039.5$ & - & DA & 17.661 & $9132 \pm 35$ & $7.94 \pm 0.07$ & $17.33 \pm 0.02$ & $17.33 \pm 0.03$ & $17.20 \pm 0.05$ & $17.49 \pm 0.12$ & 2 \\
\hline $120604.18+093022.2$ & - & $\mathrm{DA}+\mathrm{M}$ & 18.978 & $22390 \pm 627$ & $6.74 \pm 0.147$ & $18.35 \pm 0.03$ & $17.93 \pm 0.04$ & $17.51 \pm 0.06$ & $17.10 \pm 0.08$ & 3 \\
\hline $120635.92+082309.7$ & - & $\mathrm{DA}$ & 16.986 & $8422 \pm 23$ & $8.43 \pm 0.036$ & $16.62 \pm 0.01$ & $16.49 \pm 0.02$ & $16.32 \pm 0.02$ & $16.37 \pm 0.03$ & 1 \\
\hline $120924.26+095256.6$ & - & DA & 18.118 & $43636 \pm 642$ & $7.78 \pm 0.058$ & $18.30 \pm 0.04$ & $18.35 \pm 0.06$ & $18.56 \pm 0.14$ & $18.70 \pm 0.27$ & 1 \\
\hline $120957.06-012115.5$ & - & DA & 19.043 & $8032 \pm 77$ & $8.18 \pm 0.167$ & $18.64 \pm 0.06$ & $18.39 \pm 0.07$ & $18.21 \pm 0.10$ & $18.71 \pm 0.29$ & 1 \\
\hline $121033.76-004644.4$ & - & $\mathrm{DA}+\mathrm{M}$ & 17.221 & $6300 \pm 2$ & $8.93 \pm 0.067$ & $15.73 \pm 0.01$ & $15.21 \pm 0.01$ & $14.60 \pm 0.01$ & $14.33 \pm 0.01$ & 2 \\
\hline $121107.93+034216.6$ & $1208+039$ & $\mathrm{DA}+\mathrm{M}$ & 18.207 & $27938 \pm 232$ & $7.47 \pm 0.043$ & $17.31 \pm 0.02$ & $16.93 \pm 0.02$ & $16.38 \pm 0.02$ & $16.21 \pm 0.03$ & 2 \\
\hline $121114.19+104657.1$ & - & DA & 17.995 & $7835 \pm 40$ & $8.45 \pm 0.079$ & - & - & $17.09 \pm 0.04$ & $17.18 \pm 0.09$ & 1 \\
\hline $121159.41+095809.8$ & - & DA & 18.897 & $9529 \pm 74$ & $8.28 \pm 0.122$ & $18.58 \pm 0.04$ & $18.80 \pm 0.10$ & $18.53 \pm 0.13$ & $18.23 \pm 0.17$ & 1 \\
\hline $121209.30+013627.7$ & - & $\mathrm{DAH}+\mathrm{M}$ & 18.236 & $29336 \pm 324$ & $9.0 \pm 0.0010$ & $18.04 \pm 0.04$ & $17.85 \pm 0.05$ & $17.21 \pm 0.05$ & $16.21 \pm 0.03$ & 3 \\
\hline $121509.68+094847.1$ & - & DA & 18.091 & $9750 \pm 47$ & $8.71 \pm 0.082$ & $17.89 \pm 0.04$ & $17.93 \pm 0.05$ & $17.66 \pm 0.06$ & $17.85 \pm 0.14$ & 1 \\
\hline $121754.93+053829.4$ & - & DA & 18.062 & $18116 \pm 163$ & $8.15 \pm 0.034$ & $18.04 \pm 0.03$ & $18.03 \pm 0.05$ & $18.15 \pm 0.11$ & $18.02 \pm 0.19$ & 1 \\
\hline $121756.19+021046.4$ & $1215+024$ & DA & 17.420 & $75562 \pm 2039$ & $7.59 \pm 0.093$ & $17.45 \pm 0.02$ & $17.56 \pm 0.03$ & $17.67 \pm 0.08$ & $17.94 \pm 0.18$ & 1 \\
\hline $121934.05+124420.7$ & - & DA & 18.494 & $11114 \pm 86$ & $8.06 \pm 0.059$ & $18.28 \pm 0.04$ & $18.34 \pm 0.06$ & $18.45 \pm 0.11$ & $18.09 \pm 0.15$ & 1 \\
\hline $122007.05+021916.3$ & - & DA & 18.629 & $7848 \pm 59$ & $8.59 \pm 0.127$ & $18.22 \pm 0.03$ & $18.15 \pm 0.05$ & $17.90 \pm 0.09$ & $17.92 \pm 0.16$ & 1 \\
\hline $122026.44+121242.6$ & - & DA & 19.082 & $8404 \pm 71$ & $8.46 \pm 0.137$ & $18.94 \pm 0.07$ & $18.59 \pm 0.08$ & $18.32 \pm 0.10$ & $18.40 \pm 0.22$ & 1 \\
\hline $122139.78+020638.2$ & - & DA & 18.545 & $8672 \pm 42$ & $8.48 \pm 0.068$ & $18.22 \pm 0.03$ & $18.07 \pm 0.06$ & $17.99 \pm 0.11$ & $18.39 \pm 0.28$ & 1 \\
\hline $122208.47+093406.2$ & - & $\mathrm{DA}+\mathrm{M}$ & 17.830 & $6300 \pm 2$ & $7.45 \pm 0.365$ & $16.82 \pm 0.01$ & $16.43 \pm 0.01$ & $15.91 \pm 0.01$ & $15.69 \pm 0.02$ & 1 \\
\hline $122258.74+094711.6$ & - & DA & 18.346 & $21999 \pm 288$ & $7.99 \pm 0.044$ & $18.26 \pm 0.04$ & $18.44 \pm 0.07$ & $18.52 \pm 0.13$ & $18.55 \pm 0.27$ & 1 \\
\hline $122319.83+070347.9$ & - & DA & 19.083 & $7889 \pm 90$ & $8.45 \pm 0.202$ & $18.67 \pm 0.04$ & $18.53 \pm 0.07$ & $18.34 \pm 0.10$ & $18.30 \pm 0.19$ & 1 \\
\hline $122339.60-005631.2$ & - & $\mathrm{DA}+\mathrm{M}$ & 17.910 & $10966 \pm 81$ & $7.74 \pm 0.057$ & $16.74 \pm 0.01$ & $16.25 \pm 0.01$ & $15.83 \pm 0.02$ & $15.53 \pm 0.02$ & 2 \\
\hline $122752.72-015053.0$ & - & $\mathrm{DA}+\mathrm{M}$ & 18.635 & $6300 \pm 1$ & $8.8 \pm 0.14$ & $17.41 \pm 0.02$ & $17.06 \pm 0.03$ & $16.45 \pm 0.03$ & $16.29 \pm 0.04$ & 3 \\
\hline $122759.01+124051.1$ & - & DA & 18.728 & $7549 \pm 71$ & $8.69 \pm 0.199$ & $18.33 \pm 0.04$ & $18.24 \pm 0.06$ & $18.00 \pm 0.07$ & $18.09 \pm 0.16$ & 1 \\
\hline $122830.04+131723.6$ & - & DA & 18.511 & $8968 \pm 43$ & $8.08 \pm 0.077$ & $18.13 \pm 0.05$ & $18.07 \pm 0.04$ & $18.11 \pm 0.07$ & $18.12 \pm 0.16$ & 1 \\
\hline $122830.26+125635.3$ & - & DA & 16.843 & $7815 \pm 25$ & $8.48 \pm 0.047$ & $16.35 \pm 0.01$ & $16.21 \pm 0.01$ & $16.08 \pm 0.01$ & $16.08 \pm 0.03$ & 1 \\
\hline $122859.92+104033.0$ & - & DA & 16.911 & $22125 \pm 136$ & $8.22 \pm 0.021$ & $16.86 \pm 0.01$ & $16.89 \pm 0.02$ & $16.84 \pm 0.02$ & $16.42 \pm 0.04$ & 1 \\
\hline $123036.51+060040.5$ & - & DA & 19.028 & $9202 \pm 84$ & $8.79 \pm 0.137$ & $18.78 \pm 0.04$ & $18.60 \pm 0.05$ & $18.43 \pm 0.10$ & $18.55 \pm 0.23$ & 1 \\
\hline $123142.13+061020.1$ & - & DA & 18.897 & $8380 \pm 61$ & $8.31 \pm 0.117$ & $18.53 \pm 0.04$ & $18.36 \pm 0.04$ & $18.35 \pm 0.08$ & $18.17 \pm 0.17$ & 1 \\
\hline $123143.79+053155.3$ & - & DA & 17.482 & $8679 \pm 24$ & $8.39 \pm 0.038$ & $17.16 \pm 0.01$ & $17.05 \pm 0.02$ & $16.93 \pm 0.03$ & $16.85 \pm 0.05$ & 1 \\
\hline $123414.11+124829.5$ & $1231+130$ & $\mathrm{DAH}+\mathrm{DA}$ & 17.360 & $8705 \pm 29$ & $9.0 \pm 0.0010$ & $17.02 \pm 0.01$ & $17.79 \pm 0.04$ & $16.82 \pm 0.02$ & $16.78 \pm 0.05$ & 1 \\
\hline $123448.90+054718.2$ & - & DA & 17.297 & $8121 \pm 24$ & $9.0 \pm 0.011$ & $16.92 \pm 0.01$ & $16.78 \pm 0.01$ & $16.67 \pm 0.02$ & $16.68 \pm 0.05$ & 1 \\
\hline
\end{tabular}
Continued on next page 
Table C.1- continued from previous page

\begin{tabular}{|c|c|c|c|c|c|c|c|c|c|c|}
\hline SDSS J & WD & Spec. Type & $i^{\prime}$ & $T_{\text {eff }}(\mathrm{K})$ & $\log g$ & $\bar{Y}$ & $\mathrm{~J}$ & $\mathrm{H}$ & $\bar{K}$ & Ref. \\
\hline $123736.35+100029.2$ & - & $\mathrm{DA}$ & 18.685 & $7593 \pm 70$ & $8.67 \pm 0.185$ & $18.29 \pm 0.03$ & $18.05 \pm 0.04$ & $17.88 \pm 0.05$ & $17.75 \pm 0.11$ & 1 \\
\hline $124427.59+054603.3$ & - & DA & 18.410 & $10163 \pm 49$ & $8.26 \pm 0.054$ & $18.15 \pm 0.03$ & $18.01 \pm 0.04$ & $18.00 \pm 0.06$ & $17.93 \pm 0.14$ & 1 \\
\hline $124549.12+051322.3$ & - & DA & 18.675 & $7989 \pm 71$ & $8.09 \pm 0.178$ & $18.23 \pm 0.03$ & $18.19 \pm 0.04$ & $18.04 \pm 0.07$ & $18.03 \pm 0.15$ & 1 \\
\hline $125217.15+154443.1$ & $1249+160$ & DA & 15.188 & $27214 \pm 147$ & $7.28 \pm 0.017$ & $15.14 \pm 0.00$ & $15.24 \pm 0.01$ & $15.31 \pm 0.01$ & $15.45 \pm 0.02$ & 1 \\
\hline $125816.99+000710.2$ & $1255+003$ & DA & 18.662 & $14837 \pm 192$ & $7.82 \pm 0.04$ & $18.53 \pm 0.03$ & $18.64 \pm 0.07$ & $18.45 \pm 0.12$ & $18.84 \pm 0.34$ & 2 \\
\hline $130738.80+151916.2$ & - & DA & 18.373 & $17734 \pm 162$ & $7.83 \pm 0.032$ & - & - & $18.53 \pm 0.11$ & $18.26 \pm 0.16$ & 1 \\
\hline $130814.03+121705.0$ & - & $\mathrm{DA}+\mathrm{M}$ & 18.175 & $9644 \pm 65$ & $7.45 \pm 0.094$ & $16.81 \pm 0.01$ & $16.32 \pm 0.01$ & $15.89 \pm 0.01$ & $15.66 \pm 0.02$ & 3 \\
\hline $130907.15+000626.4$ & $1306+003$ & DA & 18.201 & $10825 \pm 58$ & $8.31 \pm 0.045$ & $18.03 \pm 0.02$ & $17.99 \pm 0.04$ & $18.00 \pm 0.08$ & $17.99 \pm 0.16$ & 2 \\
\hline $131056.28+144027.4$ & - & DA & 18.838 & $12616 \pm 231$ & $7.89 \pm 0.076$ & $18.76 \pm 0.04$ & $18.86 \pm 0.06$ & $18.67 \pm 0.11$ & $18.50 \pm 0.16$ & 1 \\
\hline $131409.46+132110.6$ & - & DA & 18.373 & $7685 \pm 55$ & $8.87 \pm 0.118$ & $17.93 \pm 0.02$ & $17.75 \pm 0.02$ & $17.61 \pm 0.04$ & $17.47 \pm 0.06$ & 1 \\
\hline $131641.73+122543.8$ & - & DA & 19.066 & $7831 \pm 68$ & $8.38 \pm 0.129$ & $18.53 \pm 0.04$ & $18.46 \pm 0.06$ & $18.19 \pm 0.07$ & $18.28 \pm 0.13$ & 1 \\
\hline $132001.24+112805.3$ & - & $\mathrm{DA}+\mathrm{M}$ & 16.883 & $6300 \pm 1$ & $7.04 \pm 0.102$ & $15.71 \pm 0.01$ & $15.25 \pm 0.00$ & $14.76 \pm 0.01$ & $14.52 \pm 0.01$ & 3 \\
\hline $132044.68+001855.0$ & $1318+005$ & DA & 17.695 & $19649 \pm 130$ & $8.36 \pm 0.02$ & $17.61 \pm 0.02$ & $17.62 \pm 0.04$ & $17.76 \pm 0.11$ & $17.38 \pm 0.11$ & 2 \\
\hline $132116.70+143924.7$ & - & DA & 18.355 & $16169 \pm 130$ & $7.86 \pm 0.027$ & $18.29 \pm 0.04$ & $18.28 \pm 0.06$ & $18.42 \pm 0.10$ & $18.36 \pm 0.16$ & 1 \\
\hline $132150.89+124837.1$ & - & DA & 19.022 & $9704 \pm 92$ & $8.24 \pm 0.137$ & $18.80 \pm 0.05$ & $18.68 \pm 0.06$ & $18.70 \pm 0.12$ & $18.44 \pm 0.17$ & 1 \\
\hline $132452.03+124825.4$ & - & $\mathrm{DA}+\mathrm{M}$ & 18.932 & $8167 \pm 89$ & $8.2 \pm 0.121$ & $17.66 \pm 0.02$ & $17.16 \pm 0.02$ & $16.64 \pm 0.02$ & $16.34 \pm 0.03$ & 3 \\
\hline $132652.62+000855.3$ & - & $\mathrm{DA}+\mathrm{M}$ & 18.702 & $6300 \pm 1$ & $8.77 \pm 0.16$ & $17.34 \pm 0.02$ & $16.89 \pm 0.02$ & $16.34 \pm 0.03$ & $16.12 \pm 0.04$ & 1 \\
\hline $132830.92+125941.4$ & - & $\mathrm{DA}+\mathrm{M}$ & 17.645 & $9517 \pm 32$ & $8.51 \pm 0.03$ & $16.49 \pm 0.01$ & $16.00 \pm 0.01$ & $15.54 \pm 0.01$ & $15.24 \pm 0.01$ & 3 \\
\hline $132925.21+123025.4$ & - & DA & 17.713 & $13537 \pm 138$ & $7.48 \pm 0.027$ & $17.07 \pm 0.01$ & $16.66 \pm 0.01$ & $16.27 \pm 0.02$ & $15.98 \pm 0.02$ & 1 \\
\hline $133007.57+104830.5$ & - & DA & 18.712 & $7677 \pm 63$ & $8.96 \pm 0.052$ & $18.28 \pm 0.03$ & $18.20 \pm 0.04$ & $18.08 \pm 0.08$ & $17.93 \pm 0.12$ & 1 \\
\hline $133359.97+105453.0$ & - & $\mathrm{DA}+\mathrm{M}$ & 18.496 & $6300 \pm 1$ & $8.81 \pm 0.14$ & $17.22 \pm 0.01$ & $16.73 \pm 0.01$ & $16.20 \pm 0.02$ & $15.97 \pm 0.02$ & 1 \\
\hline $133831.74-002328.0$ & - & DA & 17.443 & $11867 \pm 78$ & $8.13 \pm 0.038$ & $17.34 \pm 0.02$ & $17.30 \pm 0.03$ & $17.25 \pm 0.05$ & $17.39 \pm 0.10$ & 2 \\
\hline $133838.48-000712.5$ & - & DA & 18.918 & $10366 \pm 96$ & $8.81 \pm 0.119$ & $18.70 \pm 0.04$ & $18.85 \pm 0.12$ & $18.78 \pm 0.18$ & $19.94 \pm 0.94$ & 2 \\
\hline $133947.73-002003.5$ & - & DA & 18.026 & $8273 \pm 31$ & $8.6 \pm 0.053$ & $17.72 \pm 0.02$ & $17.56 \pm 0.04$ & $17.30 \pm 0.05$ & $17.41 \pm 0.09$ & 2 \\
\hline $134437.93+113340.3$ & - & DA & 18.463 & $23098 \pm 275$ & $8.0 \pm 0.037$ & $18.47 \pm 0.05$ & $18.49 \pm 0.06$ & $18.50 \pm 0.12$ & $18.63 \pm 0.20$ & 1 \\
\hline $134542.30+110252.5$ & - & DA & 18.614 & $10921 \pm 88$ & $8.23 \pm 0.071$ & $18.40 \pm 0.03$ & $18.42 \pm 0.05$ & $18.35 \pm 0.11$ & $18.39 \pm 0.19$ & 1 \\
\hline $140006.13+104700.4$ & - & DA & 17.696 & $9349 \pm 33$ & $8.43 \pm 0.045$ & $17.47 \pm 0.02$ & $17.35 \pm 0.02$ & $17.23 \pm 0.05$ & $17.40 \pm 0.07$ & 1 \\
\hline $140104.61-005621.6$ & - & DA & 18.841 & $7759 \pm 62$ & $7.62 \pm 0.185$ & $18.55 \pm 0.07$ & $18.79 \pm 0.16$ & $18.03 \pm 0.10$ & $18.42 \pm 0.26$ & 2 \\
\hline $140327.75+002119.5$ & $1400+005$ & DA & 17.737 & $70097 \pm 1657$ & $7.67 \pm 0.09$ & $17.87 \pm 0.02$ & $17.88 \pm 0.05$ & $18.05 \pm 0.12$ & $18.04 \pm 0.22$ & 2 \\
\hline $140353.51-014057.5$ & - & $\mathrm{DA}+\mathrm{M}$ & 20.408 & $26780 \pm 1110$ & $7.85 \pm 0.243$ & $19.11 \pm 0.10$ & $18.83 \pm 0.15$ & $18.44 \pm 0.14$ & $18.09 \pm 0.18$ & 1 \\
\hline $140506.18+012351.2$ & $1402+016$ & DA & 18.293 & $10311 \pm 55$ & $8.16 \pm 0.062$ & $17.97 \pm 0.03$ & $17.93 \pm 0.06$ & $17.89 \pm 0.09$ & $18.13 \pm 0.19$ & 2 \\
\hline $140701.61+003008.6$ & $1404+007$ & DA & 17.805 & $10294 \pm 32$ & $8.36 \pm 0.03$ & $17.63 \pm 0.03$ & $17.53 \pm 0.05$ & $17.46 \pm 0.06$ & $17.41 \pm 0.12$ & 2 \\
\hline
\end{tabular}
Continued on next page 
Table C.1- continued from previous page

\begin{tabular}{|c|c|c|c|c|c|c|c|c|c|c|}
\hline SDSS J & WD & Spec. Type & $i^{\prime}$ & $T_{\text {eff }}(\mathrm{K})$ & $\log g$ & $\bar{Y}$ & $\mathrm{~J}$ & $\mathrm{H}$ & $\bar{K}$ & Ref. \\
\hline $140723.03+003841.8$ & $1404+008$ & $\mathrm{DA}+\mathrm{M}$ & 17.024 & $8475 \pm 29$ & $8.42 \pm 0.034$ & $15.56 \pm 0.01$ & $15.00 \pm 0.01$ & $14.48 \pm 0.01$ & $14.20 \pm 0.01$ & 2 \\
\hline $141322.39-001451.5$ & - & $\mathrm{DA}$ & 18.224 & $10227 \pm 46$ & $8.95 \pm 0.055$ & $18.03 \pm 0.02$ & $17.92 \pm 0.04$ & $17.92 \pm 0.10$ & $17.88 \pm 0.18$ & 2 \\
\hline $141356.52-002643.1$ & - & DA & 17.590 & $9668 \pm 26$ & $8.48 \pm 0.029$ & $17.27 \pm 0.02$ & - & $17.13 \pm 0.05$ & $17.21 \pm 0.08$ & 2 \\
\hline $141451.73-013242.7$ & - & $\mathrm{DA}+\mathrm{M}$ & 18.806 & $9384 \pm 93$ & $7.96 \pm 0.109$ & $17.16 \pm 0.02$ & $16.56 \pm 0.02$ & $16.10 \pm 0.02$ & $15.77 \pm 0.02$ & 3 \\
\hline $141457.88+012207.3$ & $1412+016$ & DA & 17.891 & $9075 \pm 34$ & $8.15 \pm 0.062$ & $17.58 \pm 0.03$ & $17.48 \pm 0.05$ & $17.43 \pm 0.07$ & $17.64 \pm 0.13$ & 2 \\
\hline 141516.10-010912.1 & $1412-009$ & DA & 18.367 & $10163 \pm 94$ & $8.52 \pm 0.347$ & $18.19 \pm 0.04$ & $17.96 \pm 0.07$ & $17.88 \pm 0.09$ & $18.24 \pm 0.22$ & 2 \\
\hline $141708.81+005827.2$ & $1414+012$ & DA & 18.381 & $11087 \pm 76$ & $8.18 \pm 0.057$ & $18.27 \pm 0.05$ & $18.19 \pm 0.07$ & $18.03 \pm 0.10$ & $18.04 \pm 0.16$ & 2 \\
\hline $141839.65+003154.2$ & $1416+007$ & DA & 18.508 & $8889 \pm 55$ & $8.23 \pm 0.111$ & $18.23 \pm 0.04$ & $18.07 \pm 0.06$ & $17.98 \pm 0.14$ & $17.96 \pm 0.21$ & 2 \\
\hline $142120.19-004626.0$ & - & DA & 17.875 & $9039 \pm 34$ & $8.15 \pm 0.073$ & $17.30 \pm 0.02$ & $17.20 \pm 0.04$ & $17.15 \pm 0.05$ & $17.14 \pm 0.08$ & 2 \\
\hline $142433.54+031932.5$ & $1422+035$ & DA & 17.797 & $7786 \pm 35$ & $8.99 \pm 0.012$ & $17.32 \pm 0.02$ & $17.14 \pm 0.03$ & $16.99 \pm 0.04$ & $17.14 \pm 0.09$ & 2 \\
\hline $142435.03-013143.8$ & - & DA & 18.976 & $7860 \pm 66$ & $8.82 \pm 0.141$ & $18.59 \pm 0.04$ & $18.54 \pm 0.07$ & $18.18 \pm 0.10$ & $17.94 \pm 0.14$ & 1 \\
\hline $142501.59-001314.8$ & - & DA & 18.989 & $10820 \pm 91$ & $8.09 \pm 0.078$ & $18.93 \pm 0.09$ & $18.67 \pm 0.10$ & $18.66 \pm 0.17$ & $18.25 \pm 0.20$ & 2 \\
\hline $142541.31+023047.4$ & $1423+027$ & $\mathrm{DA}+\mathrm{M}$ & 18.748 & $10790 \pm 104$ & $8.11 \pm 0.091$ & $17.44 \pm 0.02$ & $17.01 \pm 0.02$ & $16.54 \pm 0.03$ & $16.21 \pm 0.04$ & 2 \\
\hline $142846.40+004526.3$ & $1426+009$ & DA & 18.643 & $9352 \pm 56$ & $8.16 \pm 0.089$ & $18.34 \pm 0.04$ & $18.42 \pm 0.07$ & $18.10 \pm 0.13$ & $17.97 \pm 0.18$ & 2 \\
\hline $143047.91+043838.6$ & $1428+048$ & DA & 17.081 & $8239 \pm 24$ & $8.51 \pm 0.036$ & $16.69 \pm 0.01$ & $16.58 \pm 0.02$ & $16.42 \pm 0.02$ & $16.43 \pm 0.04$ & 2 \\
\hline $143105.73+042215.6$ & $1428+045$ & DA & 17.468 & $22754 \pm 195$ & $7.78 \pm 0.026$ & - & $17.38 \pm 0.05$ & $17.64 \pm 0.07$ & $17.61 \pm 0.15$ & 2 \\
\hline $143249.10+014615.5$ & $1430+019$ & DA & 17.775 & $11290 \pm 73$ & $8.23 \pm 0.056$ & $17.54 \pm 0.02$ & $17.49 \pm 0.03$ & $17.59 \pm 0.09$ & $17.99 \pm 0.20$ & 2 \\
\hline $143648.99+015709.5$ & $1434+021$ & DA & 19.143 & $7729 \pm 84$ & $8.44 \pm 0.2$ & $18.76 \pm 0.06$ & $18.48 \pm 0.08$ & $18.01 \pm 0.13$ & $18.23 \pm 0.26$ & 2 \\
\hline $143654.91-014009.6$ & - & $\mathrm{DA}+\mathrm{M}$ & 19.919 & $11235 \pm 228$ & $8.3 \pm 0.136$ & $19.06 \pm 0.06$ & $18.69 \pm 0.07$ & $18.20 \pm 0.09$ & $18.33 \pm 0.20$ & 1 \\
\hline $143751.82+005254.9$ & $1435+010$ & DA & 18.138 & $18750 \pm 153$ & $7.88 \pm 0.028$ & $18.02 \pm 0.03$ & $18.11 \pm 0.06$ & $18.25 \pm 0.11$ & $18.35 \pm 0.21$ & 2 \\
\hline $143947.62-010606.7$ & $1437-008$ & $\mathrm{DA}+\mathrm{M}$ & 16.724 & $11019 \pm 120$ & $8.94 \pm 0.071$ & $15.98 \pm 0.01$ & $15.64 \pm 0.01$ & $15.14 \pm 0.01$ & $14.94 \pm 0.01$ & 2 \\
\hline $144015.58+002441.5$ & $1437+006$ & DA & 18.813 & $9990 \pm 53$ & $7.78 \pm 0.075$ & $18.67 \pm 0.06$ & $18.55 \pm 0.08$ & $18.42 \pm 0.12$ & $18.14 \pm 0.16$ & 2 \\
\hline $144108.42+011020.0$ & $1438+013$ & DA & 17.604 & $31899 \pm 182$ & $7.3 \pm 0.037$ & $17.58 \pm 0.02$ & $17.69 \pm 0.04$ & $17.67 \pm 0.08$ & $17.72 \pm 0.13$ & 2 \\
\hline $144208.95+020122.7$ & $1439+022$ & DA & 18.349 & $10028 \pm 40$ & $8.29 \pm 0.048$ & $18.14 \pm 0.04$ & $18.08 \pm 0.06$ & $17.80 \pm 0.12$ & $17.84 \pm 0.19$ & 2 \\
\hline $144242.00+021448.5$ & $1440+024$ & DA & 18.454 & $8982 \pm 45$ & $8.37 \pm 0.069$ & $18.16 \pm 0.03$ & $18.05 \pm 0.05$ & $17.91 \pm 0.12$ & $17.80 \pm 0.17$ & 2 \\
\hline $144244.18+002714.8$ & $1440+006$ & DA & 17.997 & $8211 \pm 32$ & $8.99 \pm 0.024$ & $17.66 \pm 0.02$ & $17.54 \pm 0.03$ & $17.49 \pm 0.05$ & $17.45 \pm 0.09$ & 2 \\
\hline $144433.80-005958.8$ & - & DA & 16.598 & $15745 \pm 74$ & $8.05 \pm 0.015$ & $16.47 \pm 0.01$ & $16.44 \pm 0.01$ & $16.52 \pm 0.03$ & $16.48 \pm 0.04$ & 2 \\
\hline $144810.60+015739.5$ & $1445+021$ & DA & 17.391 & $7586 \pm 27$ & $8.99 \pm 0.02$ & $17.00 \pm 0.01$ & $16.79 \pm 0.02$ & $16.66 \pm 0.04$ & $16.55 \pm 0.06$ & 2 \\
\hline $144923.37+045121.9$ & $1446+050$ & $\mathrm{DA}+\mathrm{M}$ & 20.237 & $8340 \pm 160$ & $8.81 \pm 0.161$ & $19.32 \pm 0.08$ & $18.77 \pm 0.10$ & $18.23 \pm 0.11$ & $18.23 \pm 0.21$ & 2 \\
\hline $145104.77+024355.1$ & $1448+029$ & DA & 18.101 & $8566 \pm 44$ & $8.93 \pm 0.074$ & - & $17.57 \pm 0.04$ & $17.52 \pm 0.09$ & $17.47 \pm 0.13$ & 2 \\
\hline $145300.98+005557.0$ & $1450+011$ & $\mathrm{DA}+\mathrm{M}$ & 18.540 & $9187 \pm 92$ & $7.57 \pm 0.156$ & $17.09 \pm 0.01$ & $16.58 \pm 0.02$ & $16.19 \pm 0.02$ & $15.87 \pm 0.02$ & 2 \\
\hline $145436.06-011152.4$ & $1452-009$ & DA & 17.647 & $12831 \pm 108$ & $8.34 \pm 0.031$ & $17.49 \pm 0.02$ & $17.41 \pm 0.03$ & $17.54 \pm 0.08$ & $17.66 \pm 0.13$ & 1 \\
\hline
\end{tabular}

Continued on next page 
Table C.1- continued from previous page

\begin{tabular}{|c|c|c|c|c|c|c|c|c|c|c|}
\hline SDSS J & WD & Spec. Type & $i^{\prime}$ & $T_{\text {eff }}(\mathrm{K})$ & $\log g$ & $\bar{Y}$ & $\mathrm{~J}$ & $\mathrm{H}$ & $\bar{K}$ & Ref. \\
\hline $145535.49+010246.4$ & $1453+012$ & $\mathrm{DA}$ & 19.008 & $9513 \pm 65$ & $8.16 \pm 0.111$ & $18.74 \pm 0.07$ & $18.87 \pm 0.13$ & $18.66 \pm 0.17$ & $18.52 \pm 0.27$ & 2 \\
\hline $150124.61+005430.3$ & $1458+011$ & DA & 18.279 & $10355 \pm 49$ & $8.27 \pm 0.051$ & $18.07 \pm 0.03$ & $18.13 \pm 0.04$ & $18.02 \pm 0.09$ & $18.06 \pm 0.20$ & 2 \\
\hline $150231.65+011045.8$ & $1459+013$ & $\mathrm{DA}+\mathrm{M}$ & 18.075 & $9513 \pm 57$ & $7.84 \pm 0.077$ & $16.76 \pm 0.01$ & $16.22 \pm 0.01$ & $15.70 \pm 0.01$ & $15.44 \pm 0.02$ & 2 \\
\hline $150315.52+005823.5$ & $1500+011$ & $\mathrm{DA}+\mathrm{M}$ & 19.998 & $9063 \pm 178$ & $8.77 \pm 0.183$ & $18.53 \pm 0.04$ & $18.00 \pm 0.04$ & $17.62 \pm 0.07$ & $17.00 \pm 0.08$ & 2 \\
\hline $150547.50+024839.6$ & $1503+030$ & DA & 16.976 & $18536 \pm 82$ & $7.97 \pm 0.015$ & $16.91 \pm 0.01$ & $16.96 \pm 0.02$ & $16.99 \pm 0.03$ & $17.06 \pm 0.07$ & 2 \\
\hline $150648.12+002716.4$ & $1504+006$ & $\mathrm{DA}+\mathrm{M}$ & 17.784 & $6300 \pm 1$ & $8.2 \pm 0.253$ & $16.51 \pm 0.01$ & $16.00 \pm 0.01$ & $15.46 \pm 0.01$ & $15.26 \pm 0.02$ & 2 \\
\hline $152242.25-020643.2$ & - & DA & 18.675 & $7943 \pm 69$ & $8.97 \pm 0.041$ & $18.25 \pm 0.04$ & $18.19 \pm 0.06$ & $18.14 \pm 0.13$ & $17.77 \pm 0.17$ & 1 \\
\hline $153329.88+033301.6$ & $1530+037$ & $\mathrm{DA}+\mathrm{M}$ & 18.029 & $17482 \pm 146$ & $7.91 \pm 0.029$ & $14.95 \pm 0.00$ & $14.49 \pm 0.00$ & $13.81 \pm 0.00$ & $13.64 \pm 0.00$ & 2 \\
\hline $154130.76+032312.9$ & $1539+035$ & DA & 17.167 & $16138 \pm 78$ & $7.92 \pm 0.017$ & $17.03 \pm 0.02$ & $17.10 \pm 0.03$ & $17.11 \pm 0.03$ & $17.22 \pm 0.07$ & 2 \\
\hline $154219.44+032310.9$ & $1538+035.1$ & $\mathrm{DA}+\mathrm{M}$ & 18.155 & $6300 \pm 2$ & $7.03 \pm 0.148$ & $16.65 \pm 0.01$ & $16.20 \pm 0.02$ & $15.62 \pm 0.01$ & $15.35 \pm 0.01$ & 2 \\
\hline $154254.21+061228.5$ & - & DA & 17.964 & $23618 \pm 266$ & $7.71 \pm 0.03$ & $17.94 \pm 0.03$ & $17.97 \pm 0.06$ & $18.19 \pm 0.10$ & $18.08 \pm 0.13$ & 1 \\
\hline $154431.47+060104.3$ & - & DA & 18.499 & $7946 \pm 66$ & $8.39 \pm 0.153$ & $17.96 \pm 0.03$ & $17.83 \pm 0.04$ & $17.64 \pm 0.05$ & $17.64 \pm 0.10$ & 1 \\
\hline $154436.75+054820.6$ & - & DA & 19.007 & $8753 \pm 71$ & $8.23 \pm 0.134$ & $18.67 \pm 0.05$ & $18.54 \pm 0.07$ & $18.46 \pm 0.11$ & $18.48 \pm 0.20$ & 1 \\
\hline $154832.75-001717.9$ & - & DA & 18.308 & $9933 \pm 41$ & $8.6 \pm 0.062$ & $18.08 \pm 0.02$ & $18.01 \pm 0.05$ & $17.89 \pm 0.09$ & $17.77 \pm 0.14$ & 2 \\
\hline $155359.87+082131.3$ & - & DA & 17.266 & $15904 \pm 105$ & $8.2 \pm 0.019$ & $17.21 \pm 0.02$ & $17.25 \pm 0.03$ & $17.27 \pm 0.04$ & $17.25 \pm 0.09$ & 1 \\
\hline $155720.77+091624.7$ & - & DA & 19.098 & $21990 \pm 403$ & $7.67 \pm 0.062$ & $18.82 \pm 0.04$ & $18.82 \pm 0.06$ & $19.03 \pm 0.14$ & $18.34 \pm 0.15$ & 1 \\
\hline 214645.21-003309.3 & - & DA & 18.996 & $8254 \pm 78$ & $8.91 \pm 0.098$ & $18.50 \pm 0.05$ & $18.48 \pm 0.09$ & $18.73 \pm 0.20$ & $18.30 \pm 0.22$ & 1 \\
\hline $215232.21+001447.5$ & - & $\mathrm{DA}+\mathrm{M}$ & 18.569 & $9091 \pm 75$ & $7.98 \pm 0.102$ & $17.00 \pm 0.01$ & $16.45 \pm 0.01$ & $15.96 \pm 0.02$ & $15.66 \pm 0.02$ & 3 \\
\hline $215744.78-004015.0$ & - & $\mathrm{DA}+\mathrm{M}$ & 18.987 & $6300 \pm 2$ & $8.14 \pm 0.433$ & $17.41 \pm 0.02$ & $16.87 \pm 0.02$ & $16.34 \pm 0.03$ & $15.95 \pm 0.03$ & 1 \\
\hline $220252.61+000833.4$ & - & $\mathrm{DA}+\mathrm{M}$ & 17.913 & $6300 \pm 1$ & $8.8 \pm 0.124$ & $16.54 \pm 0.01$ & $16.02 \pm 0.01$ & $15.43 \pm 0.01$ & $15.19 \pm 0.01$ & 3 \\
\hline $220338.61-001750.8$ & - & $\mathrm{DA}+\mathrm{M}$ & 18.313 & $6813 \pm 73$ & $7.68 \pm 0.218$ & $17.10 \pm 0.02$ & $16.63 \pm 0.02$ & $16.07 \pm 0.02$ & $15.81 \pm 0.02$ & 3 \\
\hline $220604.81+004635.3$ & - & $\mathrm{DA}+\mathrm{M}$ & 19.599 & $10143 \pm 120$ & $8.95 \pm 0.06$ & $18.40 \pm 0.04$ & $17.75 \pm 0.04$ & $17.17 \pm 0.05$ & $16.78 \pm 0.05$ & 1 \\
\hline $220653.42-005812.6$ & - & $\mathrm{DA}+\mathrm{M}$ & 18.741 & $6306 \pm 10$ & $6.92 \pm 0.326$ & $17.63 \pm 0.02$ & $17.21 \pm 0.03$ & $16.60 \pm 0.04$ & $16.54 \pm 0.06$ & 3 \\
\hline $220705.42-002109.6$ & - & $\mathrm{DA}+\mathrm{M}$ & 19.071 & $8481 \pm 124$ & $7.86 \pm 0.2$ & $17.61 \pm 0.02$ & $17.07 \pm 0.02$ & $16.55 \pm 0.03$ & $16.21 \pm 0.03$ & 3 \\
\hline $220841.63-000514.5$ & - & DA & 19.191 & $9224 \pm 81$ & $8.28 \pm 0.128$ & $18.16 \pm 0.04$ & $17.48 \pm 0.03$ & $17.06 \pm 0.05$ & $16.59 \pm 0.04$ & 1 \\
\hline 220946.59-001011.0 & - & $\mathrm{DA}+\mathrm{M}$ & 18.392 & $6300 \pm 2$ & $8.4 \pm 0.318$ & $17.17 \pm 0.02$ & $16.72 \pm 0.02$ & $16.13 \pm 0.02$ & $15.88 \pm 0.02$ & 3 \\
\hline $221458.36-002511.9$ & - & DA & 18.196 & $11439 \pm 78$ & $8.33 \pm 0.046$ & $18.03 \pm 0.03$ & $17.99 \pm 0.05$ & $17.97 \pm 0.13$ & $17.88 \pm 0.18$ & 1 \\
\hline $221616.59+010205.5$ & - & $\mathrm{DA}+\mathrm{M}$ & 17.904 & $11288 \pm 106$ & $7.74 \pm 0.057$ & $16.64 \pm 0.01$ & $16.15 \pm 0.01$ & $15.58 \pm 0.01$ & $15.24 \pm 0.02$ & 1 \\
\hline $221856.49+000910.4$ & - & $\mathrm{DA}+\mathrm{M}$ & 18.216 & $6300 \pm 1$ & $8.8 \pm 0.127$ & $16.72 \pm 0.01$ & $16.15 \pm 0.01$ & $15.58 \pm 0.01$ & $15.32 \pm 0.02$ & 3 \\
\hline $222030.68-004107.9$ & - & DA & 17.381 & $8037 \pm 31$ & $8.28 \pm 0.071$ & $16.95 \pm 0.01$ & $16.75 \pm 0.02$ & $16.44 \pm 0.03$ & $16.36 \pm 0.04$ & 1 \\
\hline $222108.46+002927.7$ & - & $\mathrm{DA}+\mathrm{M}$ & 17.913 & $6300 \pm 1$ & $6.98 \pm 0.105$ & $16.47 \pm 0.01$ & $15.92 \pm 0.01$ & $15.33 \pm 0.01$ & $15.06 \pm 0.01$ & 3 \\
\hline $222551.65+001637.7$ & - & DA & 18.947 & $10640 \pm 94$ & $8.16 \pm 0.09$ & $18.59 \pm 0.05$ & $18.51 \pm 0.07$ & $18.15 \pm 0.13$ & $17.60 \pm 0.13$ & 1 \\
\hline
\end{tabular}
Continued on next page 
Table C.1- continued from previous page

\begin{tabular}{|c|c|c|c|c|c|c|c|c|c|c|}
\hline SDSS J & WD & Spec. Type & $i^{\prime}$ & $T_{\text {eff }}(\mathrm{K})$ & $\log g$ & $\bar{Y}$ & $\mathrm{~J}$ & $\mathrm{H}$ & $\bar{K}$ & Ref. \\
\hline $222634.55-004144.9$ & - & $\mathrm{DA}+\mathrm{M}$ & 18.301 & $6301 \pm 4$ & $7.03 \pm 0.206$ & $16.67 \pm 0.01$ & $16.14 \pm 0.01$ & $15.58 \pm 0.01$ & $15.29 \pm 0.01$ & 3 \\
\hline $222650.26-011120.7$ & - & DA & 17.749 & $45283 \pm 692$ & $7.75 \pm 0.061$ & $17.83 \pm 0.03$ & $17.83 \pm 0.04$ & $17.95 \pm 0.09$ & $18.19 \pm 0.20$ & 1 \\
\hline 222703.34-000946.3 & - & $\mathrm{DA}+\mathrm{M}$ & 19.889 & $9650 \pm 192$ & $7.83 \pm 0.257$ & $18.70 \pm 0.05$ & $18.25 \pm 0.07$ & $17.90 \pm 0.11$ & $17.57 \pm 0.13$ & 1 \\
\hline $222838.36-002951.9$ & - & $\mathrm{DA}+\mathrm{M}$ & 17.820 & $6300 \pm 1$ & $8.65 \pm 0.166$ & - & $15.49 \pm 0.01$ & $14.90 \pm 0.01$ & $14.60 \pm 0.01$ & 3 \\
\hline $223024.37-004607.4$ & - & $\mathrm{DA}+\mathrm{M}$ & 18.055 & $6300 \pm 1$ & $8.77 \pm 0.128$ & $16.06 \pm 0.01$ & $15.42 \pm 0.01$ & $14.83 \pm 0.01$ & $14.51 \pm 0.01$ & 3 \\
\hline $223028.68-002331.2$ & - & DA & 15.575 & $9392 \pm 18$ & $8.17 \pm 0.024$ & - & $15.21 \pm 0.00$ & $15.16 \pm 0.01$ & $15.21 \pm 0.01$ & 1 \\
\hline $223120.96-000216.6$ & - & DA & 17.836 & $9179 \pm 40$ & $8.0 \pm 0.078$ & $17.55 \pm 0.02$ & $17.46 \pm 0.03$ & $17.46 \pm 0.07$ & $17.57 \pm 0.11$ & 1 \\
\hline $223421.50-002008.1$ & - & $\mathrm{DA}+\mathrm{M}$ & 19.178 & $9313 \pm 127$ & $7.53 \pm 0.197$ & $17.98 \pm 0.03$ & $17.48 \pm 0.03$ & $17.07 \pm 0.05$ & $16.85 \pm 0.06$ & 3 \\
\hline $223435.22+011248.5$ & - & $\mathrm{DA}+\mathrm{M}$ & 19.689 & $6302 \pm 7$ & $7.71 \pm 0.615$ & $18.00 \pm 0.03$ & $17.52 \pm 0.04$ & $17.01 \pm 0.04$ & $16.69 \pm 0.06$ & 1 \\
\hline 224009.07-001943.9 & - & DA & 17.602 & $15527 \pm 96$ & $7.96 \pm 0.02$ & $17.49 \pm 0.02$ & $17.49 \pm 0.03$ & $17.44 \pm 0.07$ & $17.40 \pm 0.11$ & 1 \\
\hline $224048.00+010452.5$ & - & DA & 18.699 & $7321 \pm 78$ & $8.24 \pm 0.301$ & $18.17 \pm 0.03$ & $18.08 \pm 0.05$ & $17.98 \pm 0.08$ & $17.61 \pm 0.12$ & 1 \\
\hline $224351.99+010951.1$ & - & $\mathrm{DA}+\mathrm{M}$ & 19.908 & $9689 \pm 136$ & $7.71 \pm 0.183$ & $18.75 \pm 0.05$ & $18.16 \pm 0.05$ & $17.60 \pm 0.06$ & $17.17 \pm 0.08$ & 1 \\
\hline $224522.42-000109.4$ & - & $\mathrm{DA}+\mathrm{M}$ & 19.375 & $6301 \pm 3$ & $7.61 \pm 0.597$ & $17.86 \pm 0.03$ & $17.33 \pm 0.03$ & $16.85 \pm 0.04$ & $16.52 \pm 0.05$ & 1 \\
\hline $224559.66+000505.2$ & - & DA & 18.212 & $9078 \pm 39$ & $8.34 \pm 0.064$ & $17.89 \pm 0.03$ & $17.89 \pm 0.05$ & $17.70 \pm 0.10$ & $17.70 \pm 0.15$ & 1 \\
\hline $224626.37-005909.2$ & - & DA & 18.089 & $8919 \pm 39$ & $8.29 \pm 0.08$ & $17.70 \pm 0.03$ & $17.62 \pm 0.03$ & $17.53 \pm 0.07$ & $17.67 \pm 0.13$ & 1 \\
\hline $224932.02+000645.6$ & - & $\mathrm{DA}+\mathrm{M}$ & 18.639 & $6301 \pm 3$ & $7.08 \pm 0.221$ & $16.77 \pm 0.01$ & $16.21 \pm 0.01$ & $15.63 \pm 0.02$ & $15.30 \pm 0.02$ & 1 \\
\hline $225237.53+011202.1$ & - & DA & 17.658 & $14592 \pm 177$ & $8.0 \pm 0.026$ & $17.51 \pm 0.02$ & $17.52 \pm 0.03$ & $17.57 \pm 0.07$ & $17.75 \pm 0.14$ & 1 \\
\hline 225355.94-005904.9 & - & DA & 18.395 & $10698 \pm 79$ & $8.11 \pm 0.078$ & $18.20 \pm 0.04$ & $18.17 \pm 0.06$ & $18.16 \pm 0.13$ & $18.16 \pm 0.20$ & 1 \\
\hline $225404.27+001824.1$ & - & $\mathrm{DA}+\mathrm{M}$ & 19.272 & $6301 \pm 4$ & $7.69 \pm 0.552$ & $17.47 \pm 0.02$ & $16.77 \pm 0.02$ & $16.17 \pm 0.02$ & $15.83 \pm 0.03$ & 1 \\
\hline $225503.57-001548.8$ & - & $\mathrm{DA}+\mathrm{M}$ & 18.181 & $22460 \pm 310$ & $7.73 \pm 0.043$ & $17.18 \pm 0.02$ & $16.78 \pm 0.02$ & $16.36 \pm 0.03$ & $16.02 \pm 0.03$ & 3 \\
\hline $225805.48+000925.9$ & - & DA & 17.319 & $25416 \pm 179$ & $7.89 \pm 0.023$ & $17.26 \pm 0.02$ & $17.32 \pm 0.03$ & $17.33 \pm 0.06$ & $17.38 \pm 0.10$ & 1 \\
\hline 230338.07-000847.4 & - & DA & 19.309 & $7865 \pm 101$ & $8.51 \pm 0.216$ & $18.83 \pm 0.05$ & $18.75 \pm 0.06$ & $18.61 \pm 0.13$ & $18.06 \pm 0.19$ & 2 \\
\hline $230552.08-001448.7$ & - & $\mathrm{DA}+\mathrm{M}$ & 18.386 & $6300 \pm 2$ & $8.46 \pm 0.353$ & $16.99 \pm 0.01$ & $16.47 \pm 0.01$ & $15.86 \pm 0.01$ & $15.64 \pm 0.02$ & 3 \\
\hline $230900.79+140708.9$ & - & DA & 18.330 & $18869 \pm 191$ & $7.86 \pm 0.033$ & $18.28 \pm 0.04$ & $18.31 \pm 0.08$ & $18.26 \pm 0.15$ & $18.72 \pm 0.32$ & 1 \\
\hline $230953.41+011213.2$ & $2307+009$ & DA & 17.307 & $30882 \pm 189$ & $8.59 \pm 0.025$ & $17.29 \pm 0.02$ & $17.42 \pm 0.02$ & $17.48 \pm 0.09$ & $17.80 \pm 0.16$ & 2 \\
\hline $231014.62+001440.0$ & - & $\mathrm{DA}+\mathrm{M}$ & 18.466 & $6300 \pm 1$ & $7.78 \pm 0.392$ & $16.93 \pm 0.01$ & $16.41 \pm 0.01$ & $15.85 \pm 0.01$ & $15.54 \pm 0.02$ & 3 \\
\hline $231425.79+010938.7$ & - & DA & 17.779 & $8265 \pm 35$ & $8.64 \pm 0.069$ & $17.43 \pm 0.02$ & $17.33 \pm 0.02$ & $17.13 \pm 0.06$ & $17.24 \pm 0.10$ & 1 \\
\hline $231443.95+000708.1$ & $2312-001$ & DA & 17.883 & $17746 \pm 131$ & $7.92 \pm 0.027$ & $17.80 \pm 0.02$ & $17.87 \pm 0.03$ & $17.97 \pm 0.07$ & $18.17 \pm 0.20$ & 2 \\
\hline 231645.49-010451.2 & $2314-013$ & DA & 19.219 & $9194 \pm 136$ & $8.36 \pm 0.288$ & $18.85 \pm 0.06$ & - & $18.65 \pm 0.19$ & $18.86 \pm 0.33$ & 2 \\
\hline $231731.36-001605.0$ & - & DA & 17.142 & $25100 \pm 225$ & $7.83 \pm 0.024$ & $17.10 \pm 0.01$ & $17.16 \pm 0.02$ & $17.26 \pm 0.06$ & $17.41 \pm 0.09$ & 2 \\
\hline $231814.73+003430.3$ & - & $\mathrm{DA}+\mathrm{M}$ & 18.623 & $6301 \pm 4$ & $7.16 \pm 0.27$ & $17.30 \pm 0.02$ & $16.81 \pm 0.01$ & $16.26 \pm 0.03$ & $16.07 \pm 0.04$ & 3 \\
\hline 231901.24-004949.6 & - & $\mathrm{DA}+\mathrm{M}$ & 19.265 & $9728 \pm 111$ & $8.0 \pm 0.185$ & $17.91 \pm 0.03$ & $17.33 \pm 0.03$ & $16.78 \pm 0.04$ & $16.42 \pm 0.04$ & 2 \\
\hline
\end{tabular}

Continued on next page 
Table C.1- continued from previous page

\begin{tabular}{|c|c|c|c|c|c|c|c|c|c|c|}
\hline SDSS J & WD & Spec. Type & $i^{\prime}$ & $T_{\text {eff }}(\mathrm{K})$ & $\log g$ & $\bar{Y}$ & $\mathrm{~J}$ & $\mathrm{H}$ & $\bar{K}$ & Ref. \\
\hline $231955.36+004730.4$ & $2317+005$ & $\overline{\mathrm{DA}}$ & 18.396 & $8495 \pm 56$ & $8.71 \pm 0.126$ & $18.01 \pm 0.03$ & $17.89 \pm 0.04$ & $17.78 \pm 0.11$ & $18.29 \pm 0.25$ & 2 \\
\hline $232353.90+004727.2$ & - & $\mathrm{DA}+\mathrm{M}$ & 19.473 & $6301 \pm 2$ & $8.45 \pm 0.421$ & $18.03 \pm 0.03$ & $17.53 \pm 0.03$ & $16.95 \pm 0.06$ & $16.63 \pm 0.06$ & 1 \\
\hline $232436.24-004536.3$ & - & $\mathrm{DA}+\mathrm{M}$ & 18.367 & $6300 \pm 2$ & $7.8 \pm 0.378$ & $17.06 \pm 0.02$ & $16.54 \pm 0.01$ & $15.96 \pm 0.02$ & $15.68 \pm 0.02$ & 3 \\
\hline $232448.21-005105.5$ & - & DA & 18.485 & $17904 \pm 191$ & $7.85 \pm 0.039$ & $18.41 \pm 0.04$ & $18.56 \pm 0.08$ & $18.56 \pm 0.19$ & $18.21 \pm 0.19$ & 2 \\
\hline $232531.08+154625.8$ & - & DA & 18.430 & $19422 \pm 172$ & $7.88 \pm 0.029$ & $18.40 \pm 0.04$ & $18.38 \pm 0.07$ & $18.54 \pm 0.14$ & $18.37 \pm 0.23$ & 1 \\
\hline $232539.65-002420.7$ & - & $\mathrm{DA}+\mathrm{M}$ & 19.524 & $6302 \pm 6$ & $8.11 \pm 0.711$ & $18.05 \pm 0.03$ & $17.59 \pm 0.03$ & $17.17 \pm 0.04$ & $16.73 \pm 0.05$ & 1 \\
\hline $232659.23-002348.0$ & - & $\mathrm{DA}$ & 17.740 & $10622 \pm 47$ & $8.33 \pm 0.04$ & $17.52 \pm 0.02$ & $17.61 \pm 0.05$ & $17.40 \pm 0.06$ & $17.42 \pm 0.10$ & 2 \\
\hline $232728.01+144416.5$ & - & DA & 17.842 & $8638 \pm 27$ & $8.3 \pm 0.047$ & $17.62 \pm 0.02$ & $17.45 \pm 0.03$ & $17.21 \pm 0.07$ & $17.63 \pm 0.14$ & 1 \\
\hline $232904.76+000821.2$ & - & $\mathrm{DA}+\mathrm{M}$ & 19.574 & $6302 \pm 6$ & $8.16 \pm 0.544$ & $17.61 \pm 0.02$ & $17.01 \pm 0.02$ & $16.44 \pm 0.02$ & $16.04 \pm 0.03$ & 1 \\
\hline 232913.04-001106.2 & - & $\mathrm{DA}+\mathrm{M}$ & 19.232 & $7606 \pm 95$ & $7.11 \pm 0.364$ & $17.84 \pm 0.03$ & $17.18 \pm 0.02$ & $16.60 \pm 0.03$ & $16.18 \pm 0.03$ & 1 \\
\hline $233040.47+010047.4$ & - & DA & 17.212 & $7342 \pm 31$ & $9.0 \pm 0.0090$ & $16.66 \pm 0.01$ & $16.50 \pm 0.01$ & $16.31 \pm 0.02$ & $16.23 \pm 0.03$ & 1 \\
\hline $233130.76-001128.1$ & - & DA & 18.124 & $9542 \pm 42$ & $8.55 \pm 0.055$ & $17.78 \pm 0.02$ & $17.67 \pm 0.03$ & $17.62 \pm 0.07$ & $17.52 \pm 0.10$ & 2 \\
\hline $233152.04+134206.6$ & - & $\mathrm{DA}+\mathrm{M}$ & 19.197 & $21734 \pm 375$ & $7.97 \pm 0.064$ & $18.59 \pm 0.06$ & $18.26 \pm 0.07$ & $17.75 \pm 0.11$ & $17.49 \pm 0.12$ & 3 \\
\hline $233345.97-000843.0$ & - & DA & 20.651 & $11198 \pm 433$ & $8.35 \pm 0.293$ & $19.36 \pm 0.08$ & $18.80 \pm 0.10$ & $18.22 \pm 0.13$ & $17.67 \pm 0.13$ & 1 \\
\hline $233347.65-001501.1$ & - & $\mathrm{DA}+\mathrm{M}$ & 18.654 & $6300 \pm 1$ & $8.73 \pm 0.18$ & $17.23 \pm 0.02$ & $16.77 \pm 0.02$ & $16.22 \pm 0.02$ & $15.94 \pm 0.03$ & 3 \\
\hline $233630.55-003036.0$ & - & $\mathrm{DA}+\mathrm{M}$ & 18.770 & $6300 \pm 1$ & $8.58 \pm 0.309$ & $17.14 \pm 0.01$ & $16.57 \pm 0.01$ & $15.98 \pm 0.01$ & $15.69 \pm 0.02$ & 1 \\
\hline $233807.88+000109.1$ & - & $\mathrm{DA}+\mathrm{M}$ & 18.791 & $6300 \pm 2$ & $8.82 \pm 0.14$ & $17.67 \pm 0.03$ & $17.20 \pm 0.03$ & $16.55 \pm 0.02$ & $16.36 \pm 0.04$ & 3 \\
\hline $233905.99-001754.5$ & - & $\mathrm{DA}+\mathrm{M}$ & 18.198 & $6300 \pm 1$ & $8.79 \pm 0.143$ & $16.75 \pm 0.01$ & $16.17 \pm 0.01$ & $15.65 \pm 0.01$ & $15.37 \pm 0.02$ & 3 \\
\hline $233928.35-002040.0$ & - & $\mathrm{DA}+\mathrm{M}$ & 18.075 & $6300 \pm 1$ & $8.29 \pm 0.302$ & $16.45 \pm 0.01$ & $15.86 \pm 0.01$ & $15.34 \pm 0.01$ & $15.10 \pm 0.01$ & 3 \\
\hline $234019.03+001905.2$ & - & DA & 18.802 & $7079 \pm 86$ & $8.43 \pm 0.263$ & $18.34 \pm 0.04$ & $18.08 \pm 0.07$ & $17.91 \pm 0.08$ & $17.98 \pm 0.16$ & 1 \\
\hline $234117.41-000330.3$ & - & DA & 19.132 & $12933 \pm 405$ & $7.71 \pm 0.09$ & $18.92 \pm 0.08$ & - & $18.84 \pm 0.23$ & $18.63 \pm 0.27$ & 1 \\
\hline $234131.09+003749.2$ & $2338+003$ & $\mathrm{DA}+\mathrm{M}$ & 18.355 & $6300 \pm 1$ & $8.48 \pm 0.272$ & $17.03 \pm 0.01$ & $16.52 \pm 0.02$ & $16.01 \pm 0.02$ & $15.78 \pm 0.02$ & 2 \\
\hline 234141.64-010917.2 & $2339-014$ & DA & 18.449 & $13088 \pm 173$ & $7.92 \pm 0.04$ & $18.35 \pm 0.04$ & $18.35 \pm 0.07$ & $18.38 \pm 0.12$ & $18.46 \pm 0.25$ & 2 \\
\hline $234225.67+155930.4$ & - & $\mathrm{DA}+\mathrm{M}$ & 17.518 & $10120 \pm 39$ & $7.98 \pm 0.039$ & $15.95 \pm 0.01$ & $15.44 \pm 0.01$ & $15.00 \pm 0.01$ & $14.71 \pm 0.01$ & 3 \\
\hline $234534.49-001453.7$ & - & $\mathrm{DA}+\mathrm{M}$ & 19.219 & $9492 \pm 107$ & $8.39 \pm 0.143$ & $17.99 \pm 0.03$ & $17.45 \pm 0.04$ & $16.97 \pm 0.04$ & $16.74 \pm 0.05$ & 3 \\
\hline $234621.22-003259.2$ & - & $\mathrm{DA}+\mathrm{M}$ & 19.956 & $9066 \pm 197$ & $7.6 \pm 0.354$ & $18.74 \pm 0.05$ & $18.16 \pm 0.05$ & $17.74 \pm 0.06$ & $17.43 \pm 0.09$ & 1 \\
\hline $234639.77-003716.1$ & - & DA & 18.749 & $12983 \pm 334$ & $7.97 \pm 0.083$ & - & $18.44 \pm 0.08$ & $18.57 \pm 0.18$ & $18.38 \pm 0.26$ & 2 \\
\hline $234818.15-003713.7$ & - & $\mathrm{DA}$ & 18.126 & $8468 \pm 46$ & $8.6 \pm 0.086$ & - & $17.68 \pm 0.03$ & $17.56 \pm 0.06$ & $17.34 \pm 0.09$ & 2 \\
\hline $235027.59+004358.7$ & - & $\mathrm{DA}+\mathrm{M}$ & 16.837 & $6300 \pm 2$ & $7.97 \pm 0.114$ & $15.49 \pm 0.00$ & $15.00 \pm 0.00$ & $14.54 \pm 0.01$ & $14.27 \pm 0.01$ & 1 \\
\hline $235040.74-005430.8$ & - & DA & 18.328 & $10351 \pm 60$ & $8.31 \pm 0.063$ & $18.08 \pm 0.03$ & $17.99 \pm 0.05$ & $17.77 \pm 0.07$ & $17.82 \pm 0.13$ & 2 \\
\hline $235215.44-004120.8$ & - & DA & 17.899 & $9842 \pm 46$ & $8.29 \pm 0.062$ & $17.66 \pm 0.02$ & $17.59 \pm 0.03$ & $17.44 \pm 0.05$ & $17.64 \pm 0.11$ & 2 \\
\hline $235314.79-001820.5$ & - & DA & 18.088 & $15489 \pm 148$ & $8.07 \pm 0.031$ & - & $17.98 \pm 0.05$ & $18.16 \pm 0.15$ & $18.17 \pm 0.23$ & 1 \\
\hline
\end{tabular}


Table C.2: Physical parameters and UKIDSS data for SDSS DR4 DB white dwarfs in this work.

\begin{tabular}{cccccc|ccccc}
\hline SDSS J & WD & Spec. Type & $i^{\prime}$ & $T_{\text {eff }}(\mathrm{K})$ & $\log g$ & \multicolumn{1}{c}{ Y } & K & Ref. \\
\hline $002750.01-001023.4$ & - & DB+M & 17.09 & $6300 \pm 1.0$ & $8.84 \pm 0.11$ & $15.59 \pm 0.01$ & $15.05 \pm 0.01$ & $14.56 \pm 0.01$ & $14.25 \pm 0.01$ & 3 \\
$012148.20-001053.3$ & - & DB & 16.78 & $16890 \pm 68.0$ & $8.26 \pm 0.04$ & $16.73 \pm 0.01$ & $16.77 \pm 0.02$ & $16.82 \pm 0.03$ & $16.99 \pm 0.06$ & 1 \\
$033320.38+000720.6$ & $0330-000$ & DBH & 16.38 & $10086 \pm 16.0$ & $8.32 \pm 0.21$ & $16.01 \pm 0.01$ & $15.93 \pm 0.01$ & $15.88 \pm 0.01$ & $15.84 \pm 0.03$ & 2 \\
$091029.42+090205.1$ & - & DB & 17.62 & $16711 \pm 55.0$ & $8.24 \pm 0.03$ & $17.55 \pm 0.02$ & $17.57 \pm 0.02$ & $17.66 \pm 0.04$ & $17.84 \pm 0.09$ & 1 \\
$092452.73+020712.2$ & $0922+023$ & DB & 18.97 & $15231 \pm 147.0$ & $8.49 \pm 0.10$ & $20.80 \pm 0.36$ & - & $18.20 \pm 0.12$ & $17.60 \pm 0.11$ & 2 \\
$095102.22+010432.5$ & $0948+013$ & DB & 16.16 & $17622 \pm 25.0$ & $8.22 \pm 0.02$ & $16.1 \pm 0.01$ & $16.17 \pm 0.01$ & $16.23 \pm 0.02$ & $16.31 \pm 0.03$ & 2 \\
$095353.91+074246.1$ & - & DB & 18.24 & $19019 \pm 179.0$ & $8.17 \pm 0.05$ & $18.16 \pm 0.03$ & $18.26 \pm 0.03$ & $18.28 \pm 0.07$ & $18.39 \pm 0.16$ & 1 \\
$124112.98+122613.7$ & $1238+127$ & DB & 17.55 & $16149 \pm 72.0$ & $8.33 \pm 0.05$ & $17.47 \pm 0.02$ & $17.53 \pm 0.03$ & $17.59 \pm 0.05$ & $17.60 \pm 0.11$ & 1 \\
$135541.69+110031.7$ & - & DB & 18.53 & $26866 \pm 888.0$ & $7.86 \pm 0.06$ & $18.40 \pm 0.03$ & $18.42 \pm 0.05$ & $18.52 \pm 0.11$ & $18.79 \pm 0.22$ & 1 \\
$144258.47+001031.5$ & $1440+003$ & DB+M & 17.47 & $15580 \pm 125.0$ & $7.53 \pm 0.08$ & $15.86 \pm 0.01$ & $15.29 \pm 0.01$ & $14.7 \pm 0.01$ & $14.42 \pm 0.01$ & 2 \\
\hline
\end{tabular}


Table C.3: Physical parameters and UKIDSS data for McCook \& Sion

DA white dwarfs in this work.

\begin{tabular}{|c|c|c|c|c|c|c|c|c|c|c|}
\hline WD & Name & Spec. Type & $i^{\prime}$ & $T_{\text {eff }}(\mathrm{K})$ & $\log g$ & $\mathrm{Y}$ & $\mathrm{J}$ & $\mathrm{H}$ & $\mathrm{K}$ & Ref. \\
\hline WD0014+003 & - & $\mathrm{DA}+\mathrm{M}$ & 18.09 & - & - & $16.38 \pm 0.01$ & $15.85 \pm 0.01$ & $15.28 \pm 0.01$ & $14.97 \pm 0.01$ & \\
\hline WD0155+069 & GSC 00036-00907 & DA & $V=15.22$ & 20600 & 7.72 & $15.76 \pm 0.01$ & - & $15.87 \pm 0.02$ & $16.01 \pm 0.03$ & 3 \\
\hline WD0225+004 & LP 590-143 & DA & 17.76 & $\sim 6300$ & - & $17.17 \pm 0.02$ & $16.88 \pm 0.02$ & $16.54 \pm 0.03$ & $16.61 \pm 0.05$ & 1 \\
\hline WD0227+050 & NSV 843 & DA & $V=12.65$ & 19085 & 7.78 & $13.18 \pm 0.00$ & $13.25 \pm 0.00$ & $13.33 \pm 0.00$ & $13.40 \pm 0.00$ & 2 \\
\hline WD0235+052 & - & DA & $V=17.8$ & 16000 & 7.63 & $17.32 \pm 0.02$ & $17.37 \pm 0.03$ & $17.44 \pm 0.06$ & $17.57 \pm 0.12$ & 3 \\
\hline WD0838+035 & - & DA & 15.02 & 38500 & 7.78 & $15.06 \pm 0.00$ & $15.17 \pm 0.01$ & $15.24 \pm 0.01$ & $15.39 \pm 0.02$ & 4 \\
\hline WD0928+085 & - & DA & 17.49 & 29430 & 8.56 & $17.51 \pm 0.02$ & $17.64 \pm 0.03$ & $17.67 \pm 0.04$ & $17.69 \pm 0.11$ & 5 \\
\hline WD0933+025 & - & $\mathrm{DA}+\mathrm{M}$ & 15.25 & - & - & $13.86 \pm 0.00$ & $13.30 \pm 0.00$ & $12.77 \pm 0.00$ & $12.49 \pm 0.00$ & \\
\hline WD0939+071 & - & DA & 14.71 & 7770 & - & $14.15 \pm 0.00$ & $13.93 \pm 0.00$ & $13.66 \pm 0.00$ & $13.60 \pm 0.00$ & 11 \\
\hline WD0950+077 & - & DA & 16.33 & 14770 & 7.95 & $16.24 \pm 0.01$ & $16.28 \pm 0.01$ & $16.35 \pm 0.02$ & $16.41 \pm 0.03$ & 12 \\
\hline WD0956+020 & - & DA & 16.16 & 15550 & 7.75 & $16.03 \pm 0.01$ & $16.06 \pm 0.01$ & $16.13 \pm 0.02$ & $16.18 \pm 0.03$ & 2 \\
\hline WD0956+045 & - & DA & 16.46 & 18150 & 7.81 & - & - & $14.19 \pm 0.00$ & $13.87 \pm 0.01$ & 12 \\
\hline WD1003-023 & PG 1003-023 & DA & 15.77 & 20340 & 7.95 & $15.70 \pm 0.01$ & $15.76 \pm 0.01$ & $15.79 \pm 0.01$ & $15.89 \pm 0.03$ & 2 \\
\hline WD1012-008 & - & DA & 16.06 & 22270 & 7.98 & $16.07 \pm 0.01$ & $16.14 \pm 0.01$ & $16.21 \pm 0.02$ & $16.31 \pm 0.04$ & 13 \\
\hline WD1015+076 & - & $\mathrm{DA}+\mathrm{M}$ & 15.51 & - & - & $14.81 \pm 0.00$ & $14.56 \pm 0.00$ & $14.24 \pm 0.00$ & $14.16 \pm 0.01$ & \\
\hline WD1112-012 & - & DA & $V=18.3$ & - & - & $18.60 \pm 0.05$ & $18.35 \pm 0.07$ & $18.53 \pm 0.21$ & $18.22 \pm 0.23$ & \\
\hline WD1124+017 & - & DA & 16.69 & 15868 & 7.69 & $16.59 \pm 0.01$ & $16.68 \pm 0.02$ & $16.71 \pm 0.04$ & $16.75 \pm 0.06$ & 13 \\
\hline WD1130-013 & - & $\mathrm{DA}+\mathrm{M}$ & 18.23 & - & - & - & $16.31 \pm 0.01$ & $15.74 \pm 0.01$ & $15.46 \pm 0.02$ & \\
\hline WD1144+011 & - & $\mathrm{DA}+\mathrm{Me}$ & 16.13 & - & - & $14.47 \pm 0.00$ & $13.93 \pm 0.00$ & $13.42 \pm 0.00$ & $13.13 \pm 0.00$ & 1 \\
\hline WD1155-011 & - & $\mathrm{DA}$ & 19.62 & & - & $18.26 \pm 0.03$ & $17.83 \pm 0.03$ & $17.22 \pm 0.03$ & $16.89 \pm 0.06$ & 1 \\
\hline WD1210+140 & - & DA & 15.30 & 31930 & 7.03 & $15.26 \pm 0.00$ & $15.36 \pm 0.01$ & $15.44 \pm 0.01$ & $15.59 \pm 0.02$ & 5 \\
\hline WD1214+146 & - & DA & 18.33 & $\sim 13400$ & - & $18.20 \pm 0.03$ & $18.12 \pm 0.05$ & $18.38 \pm 0.13$ & $17.96 \pm 0.17$ & 1 \\
\hline WD1225+006 & - & DA & 15.10 & 9530 & 8.08 & - & $14.75 \pm 0.00$ & $14.70 \pm 0.01$ & $14.76 \pm 0.01$ & 13 \\
\hline WD1226+158 & - & DA & 17.97 & $\sim 12800$ & - & - & - & $18.02 \pm 0.11$ & $18.38 \pm 0.25$ & 1 \\
\hline WD1231+129 & - & DA & 18.02 & $\sim 13800$ & - & $18.08 \pm 0.03$ & $18.07 \pm 0.05$ & $17.94 \pm 0.07$ & $18.21 \pm 0.18$ & 1 \\
\hline WD1232+135 & - & $\mathrm{DA}+\mathrm{M}$ & 15.92 & - & - & $14.63 \pm 0.00$ & $14.14 \pm 0.00$ & $13.52 \pm 0.00$ & $13.32 \pm 0.00$ & \\
\hline WD1257+047 & - & DA & 15.46 & 22320 & - & $15.45 \pm 0.00$ & $15.51 \pm 0.01$ & $15.55 \pm 0.01$ & $15.62 \pm 0.02$ & 5 \\
\hline WD1312+098 & - & DAH & 16.90 & 15000 & - & $16.88 \pm 0.01$ & $16.92 \pm 0.02$ & $16.97 \pm 0.04$ & $17.13 \pm 0.08$ & 14 \\
\hline WD1349+144 & PB 4117 & DA & 15.67 & 16620 & 7.68 & $15.59 \pm 0.01$ & $15.59 \pm 0.01$ & $15.69 \pm 0.01$ & $15.74 \pm 0.02$ & 12 \\
\hline WD1411+135 & - & DA & 16.97 & $\sim 19600$ & - & $16.90 \pm 0.01$ & $16.96 \pm 0.01$ & $17.00 \pm 0.02$ & $17.11 \pm 0.05$ & 1 \\
\hline
\end{tabular}


Table C.3- continued from previous page

\begin{tabular}{|c|c|c|c|c|c|c|c|c|c|c|}
\hline WD & Name & Spec. Type & $i^{\prime}$ & $T_{\text {eff }}(\mathrm{K})$ & $\log g$ & $\bar{Y}$ & $\mathrm{~J}$ & $\mathrm{H}$ & $\mathrm{K}$ & Ref. \\
\hline WD1413+003 & - & $\overline{\mathrm{DA}}$ & 16.46 & 14590 & 8.05 & $16.42 \pm 0.01$ & $16.40 \pm 0.01$ & $16.41 \pm 0.03$ & $16.47 \pm 0.05$ & 13 \\
\hline WD1413+015 & - & $\mathrm{DA}+\mathrm{M}$ & 17.52 & 48800 & 7.70 & $17.23 \pm 0.02$ & $17.59 \pm 0.05$ & $16.63 \pm 0.03$ & $16.55 \pm 0.05$ & 12 \\
\hline WD1415+132 & Feige 93 & $\mathrm{DA}+\mathrm{M}$ & 15.50 & 34000 & 7.43 & $14.67 \pm 0.00$ & $14.26 \pm 0.00$ & $13.82 \pm 0.00$ & $13.55 \pm 0.00$ & \\
\hline WD1422+028 & - & DA & 17.11 & $\sim 18400$ & & $17.06 \pm 0.01$ & $17.13 \pm 0.02$ & $17.22 \pm 0.06$ & $17.25 \pm 0.11$ & 1 \\
\hline WD1428+102 & - & DA & 17.34 & $\sim 27100$ & & $17.34 \pm 0.01$ & $17.35 \pm 0.02$ & $17.38 \pm 0.04$ & $17.58 \pm 0.08$ & 1 \\
\hline WD1501+032 & - & DA & 15.94 & 13770 & 7.88 & $15.84 \pm 0.01$ & $15.86 \pm 0.01$ & $15.91 \pm 0.01$ & $15.98 \pm 0.03$ & 5 \\
\hline WD1527+090 & - & DA & 15.25 & 21520 & 8.02 & $14.78 \pm 0.00$ & $14.84 \pm 0.00$ & $14.90 \pm 0.01$ & $15.00 \pm 0.01$ & 5 \\
\hline WD1544+008 & - & DA & $V=15.27$ & 43374 & 7.47 & $15.69 \pm 0.01$ & $15.73 \pm 0.01$ & $15.81 \pm 0.01$ & $15.92 \pm 0.03$ & 10 \\
\hline WD1547+015 & - & DA & $V=15.9$ & 76910 & 7.57 & $16.44 \pm 0.01$ & $16.49 \pm 0.01$ & $16.52 \pm 0.03$ & $16.66 \pm 0.06$ & 5 \\
\hline WD1547+057 & - & DA & 16.43 & 24850 & 8.41 & $16.44 \pm 0.01$ & $16.48 \pm 0.01$ & $16.60 \pm 0.03$ & $16.61 \pm 0.04$ & 2 \\
\hline WD2314+064 & - & DA & $V=15.93$ & 17570 & 7.98 & $16.51 \pm 0.01$ & $16.50 \pm 0.01$ & $16.52 \pm 0.02$ & $16.59 \pm 0.04$ & 2 \\
\hline WD2314+141 & - & DA & 17.34 & $\sim 20600$ & - & $17.26 \pm 0.02$ & $17.35 \pm 0.04$ & $17.43 \pm 0.07$ & $17.56 \pm 0.11$ & 1 \\
\hline WD2318+126 & - & DA & $V=15.9$ & - & - & $16.58 \pm 0.01$ & $16.58 \pm 0.01$ & $16.58 \pm 0.04$ & $16.65 \pm 0.05$ & \\
\hline WD2336+063 & - & DA & $V=15.6$ & 16520 & 8.03 & $15.92 \pm 0.01$ & $15.98 \pm 0.01$ & $16.05 \pm 0.02$ & $16.09 \pm 0.04$ & 5 \\
\hline
\end{tabular}

${ }^{1}$ This Work, ${ }^{2}$ Holberg \& Bergeron (2006), ${ }^{3}$ Homeier et al. (1998), ${ }^{4}$ Finley (1997), ${ }^{5}$ Liebert, Bergeron \& Holberg (2004), ${ }^{6}$ Werner et al. (1997), ${ }^{7}$ Koester \& Knist (2009), ${ }^{8}$ Dufour et al. (2005), ${ }^{9}$ Voss et al. (2007), ${ }^{10}$ Sion et al. (1988), ${ }^{11}$ Schroder, Pauli \& Napiwotzki (2004), ${ }^{12}$ Liebert, Bergeron \& Holberg (2005), ${ }^{13}$ Koester et al. (2001), ${ }^{14}$ Liebert, Bergeron \& Holberg (2003), ${ }^{15}$ Friedrich et al. (2000) 


\section{Bibliography}

Allard, F., Hauschildt, P. H., Alexander, D. R. \& Starrfield, S. (1997), 'Model Atmospheres of Very Low Mass Stars and Brown Dwarfs', ARA\&A 35, 137-177.

Baraffe, I. \& Chabrier, G. (1996), 'Mass-Spectral Class Relationship for M Dwarfs', ApJL 461, L51.

Baraffe, I., Chabrier, G., Allard, F. \& Hauschildt, P. H. (1998), 'Evolutionary models for solar metallicity low-mass stars: mass-magnitude relationships and color-magnitude diagrams', $A \& A$ 337, 403-412.

Baraffe, I., Chabrier, G., Allard, F. \& Hauschildt, P. H. (2002), 'Evolutionary models for low-mass stars and brown dwarfs: Uncertainties and limits at very young ages', $A \& A$ 382, 563-572.

Baraffe, I., Chabrier, G., Barman, T. S., Allard, F. \& Hauschildt, P. H. (2003), 'Evolutionary models for cool brown dwarfs and extrasolar giant planets. The case of HD 209458', A\&A 402, 701-712.

Barstow, M. A., Good, S. A., Holberg, J. B., Hubeny, I., Bannister, N. P., Bruhweiler, F. C., Burleigh, M. R. \& Napiwotzki, R. (2003), 'Heavy-element abundance patterns in hot DA white dwarfs', MNRAS 341, 870-890.

Becklin, E. E., Farihi, J., Jura, M., Song, I., Weinberger, A. J. \& Zuckerman, B. (2005), 'A Dusty Disk around GD 362, a White Dwarf with a Uniquely High Photospheric Metal Abundance', ApJL 632, L119-L122.

Becklin, E. E. \& Zuckerman, B. (1988), 'A low-temperature companion to a white dwarf star', Nature $336,656-658$.

Bergeron, P., Ruiz, M. T. \& Leggett, S. K. (1997), 'The Chemical Evolution of Cool White Dwarfs and the Age of the Local Galactic Disk', ApJS 108, 339.

Bergeron, P., Saffer, R. A. \& Liebert, J. (1992), 'A spectroscopic determination of the mass distribution of DA white dwarfs', ApJ 394, 228-247.

Bergeron, P., Wesemael, F., Lamontagne, R., Fontaine, G., Saffer, R. A. \& Allard, N. F. (1995), 'Optical and Ultraviolet Analyses of ZZ Ceti Stars and Study of the Atmospheric Convective Efficiency in DA White Dwarfs', ApJ 449, 258.

Brinkworth, C. S., Gänsicke, B. T., Marsh, T. R., Hoard, D. W. \& Tappert, C. (2009), 'A Dusty Component to the Gaseous Debris Disk Around the White Dwarf SDSS J1228+1040', ApJ 696, 1402-1406.

Burgasser, A. J., Geballe, T. R., Leggett, S. K., Kirkpatrick, J. D. \& Golimowski, D. A. (2006), 'A Unified Near-Infrared Spectral Classification Scheme for T Dwarfs', ApJ 637, 1067-1093.

Burleigh, M. R., Clarke, F. J. \& Hodgkin, S. T. (2002), 'Imaging planets around nearby white dwarfs', MNRAS 331, L41-L45.

Burleigh, M. R., Hogan, E., Dobbie, P. D., Napiwotzki, R. \& Maxted, P. F. L. (2006a), 'A near-infrared spectroscopic detection of the brown dwarf in the post common envelope binary WD0137-349', MNRAS 373, L55-L59. 
Burleigh, M. R., Marsh, T. R., Gänsicke, B. T., Goad, M. R., Dhillon, V. S., Littlefair, S. P., Wells, M., Bannister, N. P., Hurkett, C. P., Martindale, A., Dobbie, P. D., Casewell, S. L., Baker, D. E. A., Duke, J., Farihi, J., Irwin, M. J., Hewett, P. C., Roche, P. \& Lewis, F. (2006b), 'The nature of the close magnetic white dwarf + probable brown dwarf binary SDSSJ121209.31+013627.7', MNRAS 373, 1416-1422.

Burrows, A., Hubbard, W. B., Lunine, J. I. \& Liebert, J. (2001), 'The theory of brown dwarfs and extrasolar giant planets', Reviews of Modern Physics 73, 719-765.

Caballero, J. A., Burgasser, A. J. \& Klement, R. (2008), 'Contamination by field late-M, L, and T dwarfs in deep surveys', $A \& A$ 488, 181-190.

Carpenter, J. M. (2001), 'Color Transformations for the 2MASS Second Incremental Data Release', $A J$ $121,2851-2871$.

Casali, M., Adamson, A., Alves de Oliveira, C., Almaini, O., Burch, K., Chuter, T., Elliot, J., Folger, M., Foucaud, S., Hambly, N., Hastie, M., Henry, D., Hirst, P., Irwin, M., Ives, D., Lawrence, A., Laidlaw, K., Lee, D., Lewis, J., Lunney, D., McLay, S., Montgomery, D., Pickup, A., Read, M., Rees, N., Robson, I., Sekiguchi, K., Vick, A., Warren, S. \& Woodward, B. (2007), 'The UKIRT wide-field camera', $A \& A$ 467, 777-784.

Casewell, S. L., Dobbie, P. D., Napiwotzki, R., Burleigh, M. R., Barstow, M. A. \& Jameson, R. F. (2009), 'High-resolution optical spectroscopy of Praesepe white dwarfs', MNRAS 395, 1795-1804.

Chabrier, G., Baraffe, I., Allard, F. \& Hauschildt, P. (2000), 'Evolutionary Models for Very Low-Mass Stars and Brown Dwarfs with Dusty Atmospheres', ApJ 542, 464-472.

Chandrasekhar, S. (1931), 'The Maximum Mass of Ideal White Dwarfs', ApJ 74, 81.

Claver, C. F., Liebert, J., Bergeron, P. \& Koester, D. (2001), 'The Masses of White Dwarfs in the Praesepe Open Cluster', ApJ 563, 987-998.

Cushing, M. C., Rayner, J. T. \& Vacca, W. D. (2005), 'An Infrared Spectroscopic Sequence of M, L, and T Dwarfs', ApJ 623, 1115-1140.

Cushing, M. C., Vacca, W. D. \& Rayner, J. T. (2004), 'Spextool: A Spectral Extraction Package for SpeX, a 0.8-5.5 Micron Cross-Dispersed Spectrograph', PASP 116, 362-376.

Day-Jones, A., Pinfield, D. J., Jones, H. R. A., Napiwotzki, R., Burningham, B., Jenkins, J. S. \& UKIDSS Cool Dwarf Science Working Group (2008), Hunting For Wild Brown Dwarf Companions To White Dwarfs In UKIDSS And SDSS, in 'American Astronomical Society Meeting Abstracts', Vol. 211 of American Astronomical Society Meeting Abstracts, p. 103.30.

de Kool, M. (1990), 'Common envelope evolution and double cores of planetary nebulae', ApJ 358, 189195.

de Kool, M. (1992), 'Statistics of cataclysmic variable formation', A\&A 261, 188-202.

Debes, J. H., López-Morales, M., Bonanos, A. Z. \& Weinberger, A. J. (2006), 'Detection of a Variable Infrared Excess around SDSS J121209.31+013627.7’, ApJL 647, L147-L150.

Debes, J. H., Sigurdsson, S. \& Hansen, B. (2007), 'Cool Customers in the Stellar Graveyard. IV. Spitzer Search for Mid-IR excesses Around Five DAs', AJ 134, 1662-1670.

Debes, J. H., Sigurdsson, S. \& Woodgate, B. E. (2005), 'Cool Customers in the Stellar Graveyard. II. Limits to Substellar Objects around Nearby DAZ White Dwarfs', AJ 130, 1221-1230.

Dobbie, P. D., Burleigh, M. R., Levan, A. J., Barstow, M. A., Napiwotzki, R., Holberg, J. B., Hubeny, I. \& Howell, S. B. (2005), 'A near-infrared spectroscopic search for very-low-mass cool companions to notable DA white dwarfs', MNRAS 357, 1049-1058.

Dobbie, P. D., Napiwotzki, R., Burleigh, M. R., Barstow, M. A., Boyce, D. D., Casewell, S. L., Jameson, R. F., Hubeny, I. \& Fontaine, G. (2006), 'New Praesepe white dwarfs and the initial mass-final mass relation', MNRAS 369, 383-389. 
Dobbie, P. D., Pinfield, D. J., Napiwotzki, R., Hambly, N. C., Burleigh, M. R., Barstow, M. A., Jameson, R. F. \& Hubeny, I. (2004), 'Praesepe and the seven white dwarfs', MNRAS 355, L39-L43.

D’Odorico, S., Dekker, H., Mazzoleni, R., Vernet, J., Guinouard, I., Groot, P., Hammer, F., Rasmussen, P. K., Kaper, L., Navarro, R., Pallavicini, R., Peroux, C. \& Zerbi, F. M. (2006), X-shooter UV- to K-band intermediate-resolution high-efficiency spectrograph for the VLT: status report at the final design review, in 'Society of Photo-Optical Instrumentation Engineers (SPIE) Conference Series', Vol. 6269 of Presented at the Society of Photo-Optical Instrumentation Engineers (SPIE) Conference.

Dupuy, T. J., Liu, M. C. \& Ireland, M. J. (2009), 'Dynamical Mass of the Substellar Benchmark Binary HD 130948BC', ApJ 692, 729-752.

Dye, S., Warren, S. J., Hambly, N. C., Cross, N. J. G., Hodgkin, S. T., Irwin, M. J., Lawrence, A., Adamson, A. J., Almaini, O., Edge, A. C., Hirst, P., Jameson, R. F., Lucas, P. W., van Breukelen, C., Bryant, J., Casali, M., Collins, R. S., Dalton, G. B., Davies, J. I., Davis, C. J., Emerson, J. P., Evans, D. W., Foucaud, S., Gonzales-Solares, E. A., Hewett, P. C., Kendall, T. R., Kerr, T. H., Leggett, S. K., Lodieu, N., Loveday, J., Lewis, J. R., Mann, R. G., McMahon, R. G., Mortlock, D. J., Nakajima, Y., Pinfield, D. J., Rawlings, M. G., Read, M. A., Riello, M., Sekiguchi, K., Smith, A. J., Sutorius, E. T. W., Varricatt, W., Walton, N. A. \& Weatherley, S. J. (2006), 'The UKIRT Infrared Deep Sky Survey Early Data Release', MNRAS 372, 1227-1252.

Eisenstein, D. J., Liebert, J., Harris, H. C., Kleinman, S. J., Nitta, A., Silvestri, N., Anderson, S. A., Barentine, J. C., Brewington, H. J., Brinkmann, J., Harvanek, M., Krzesiński, J., Neilsen, Jr., E. H., Long, D., Schneider, D. P. \& Snedden, S. A. (2006), 'A Catalog of Spectroscopically Confirmed White Dwarfs from the Sloan Digital Sky Survey Data Release 4', ApJS 167, 40-58.

Emerson, J. P., Sutherland, W. J., McPherson, A. M., Craig, S. C., Dalton, G. B. \& Ward, A. K. (2004), 'The Visible and Infrared Survey Telescope for Astronomy', The Messenger 117, 27-32.

Farihi, J., Becklin, E. E. \& Zuckerman, B. (2005), 'Low-Luminosity Companions to White Dwarfs', ApJS $161,394-428$.

Farihi, J., Becklin, E. E. \& Zuckerman, B. (2006), 'Low-luminosity companions to white dwarfs (Farihi+, 2005)', VizieR Online Data Catalog 216, 10394.

Farihi, J., Burleigh, M. R. \& Hoard, D. W. (2008), 'A Near-Infrared Spectroscopic Study of the Accreting Magnetic White Dwarf SDSS J121209.31+013627.7 and Its Substellar Companion’, ApJ 674, 421430.

Farihi, J. \& Christopher, M. (2004), 'A Possible Brown Dwarf Companion to the White Dwarf GD 1400', AJ 128, 1868-1871.

Farihi, J., Hoard, D. W. \& Wachter, S. (2006a), 'White Dwarf-Red Dwarf Systems Resolved with the Hubble Space Telescope. I. First Results’, ApJ 646, 480.

Farihi, J., Hoard, D. W. \& Wachter, S. (2006b), 'White Dwarf-Red Dwarf Systems Resolved with the Hubble Space Telescope. I. First Results', ApJ 646, 480-492.

Farihi, J., Jura, M. \& Zuckerman, B. (2009), 'Infrared Signatures of Disrupted Minor Planets at White Dwarfs', ApJ 694, 805-819.

Feline, W. J., Dhillon, V. S., Marsh, T. R. \& Brinkworth, C. S. (2004), 'ULTRACAM photometry of the eclipsing cataclysmic variables XZ Eri and DV UMa', MNRAS 355, 1-10.

Ferrario, L., Wickramasinghe, D., Liebert, J. \& Williams, K. A. (2005), 'The open-cluster initial-final mass relationship and the high-mass tail of the white dwarf distribution', MNRAS 361, 1131-1135.

Finley, D. S. (1997), The Chemical Composition of Massive DA White Dwarfs, in 'EUVE Proposal', p. 59.

Fontaine, G., Brassard, P. \& Bergeron, P. (2001), 'The Potential of White Dwarf Cosmochronology', PASP 113, 409-435. 
Fowler, R. H. (1926), 'On Dense Matter', MNRAS 87, 114-112.

Gänsicke, B. T., Koester, D., Marsh, T. R., Rebassa-Mansergas, A. \& Southworth, J. (2008), 'SDSSJ084539.17+225728.0: the first DBZ white dwarf with a metal-rich gaseous debris disc', MNRAS 391, L103-L107.

Gänsicke, B. T., Marsh, T. R. \& Southworth, J. (2007), 'SDSSJ104341.53+085558.2: A second white dwarf with a gaseous debris disc', ArXiv e-prints 705.

Gänsicke, B. T., Marsh, T. R., Southworth, J. \& Rebassa-Mansergas, A. (2006), 'A Gaseous Metal Disk Around a White Dwarf', Science 314, 1908.

Girardi, L., Bressan, A., Bertelli, G. \& Chiosi, C. (2000), 'Evolutionary tracks and isochrones for low- and intermediate-mass stars: From 0.15 to $7 \mathrm{Ms}$, and from $\mathrm{Z}=0.0004$ to 0.03 ', AAS 141, 371-383.

Golimowski, D. A., Leggett, S. K., Marley, M. S., Fan, X., Geballe, T. R., Knapp, G. R., Vrba, F. J., Henden, A. A., Luginbuhl, C. B., Guetter, H. H., Munn, J. A., Canzian, B., Zheng, W., Tsvetanov, Z. I., Chiu, K., Glazebrook, K., Hoversten, E. A., Schneider, D. P. \& Brinkmann, J. (2004), 'L' and M'Photometry of Ultracool Dwarfs', AJ 127, 3516-3536.

González-Lópezlira, R. A., Bruzual-A., G., Charlot, S., Ballesteros-Paredes, J. \& Loinard, L. (2010), 'Tracers of stellar mass loss - I. Optical and near-IR colours and surface brightness fluctuations', MNRAS 403, 1213-1238.

Green, P. J., Ali, B. \& Napiwotzki, R. (2000a), 'Cool Companions to Hot White Dwarfs', ApJ 540, 992.

Green, P. J., Ali, B. \& Napiwotzki, R. (2000b), 'Cool Companions to Hot White Dwarfs', ApJ 540, 9921004.

Green, R. F., Schmidt, M. \& Liebert, J. (1986), 'The Palomar-Green catalog of ultraviolet-excess stellar objects', ApJS 61, 305-352.

Grether, D. \& Lineweaver, C. H. (2006), 'How Dry is the Brown Dwarf Desert? Quantifying the Relative Number of Planets, Brown Dwarfs, and Stellar Companions around Nearby Sun-like Stars', ApJ 640, 1051-1062.

Guseinov, O. K., Novruzova, K. I. \& Rustamov, I. S. (1983), 'Atmospheric parameters and mass distribution of DA-white dwarfs', ApSS 96, 1-23.

Haro, G. \& Luyten, W. J. (1962), 'Faint blue stars in the region near the South Galactic Pole.', Boletin del Instituto de Tonantzintla 3, 37-117.

Herwig, F., Blöcker, T., Langer, N. \& Driebe, T. (1999), 'On the formation of hydrogen-deficient postAGB stars', $A \& A$ 349, L5-L8.

Hewett, P. C., Warren, S. J., Leggett, S. K. \& Hodgkin, S. T. (2006), 'The UKIRT Infrared Deep Sky Survey ZY JHK photometric system: passbands and synthetic colours', MNRAS 367, 454-468.

Hoard, D. W., Wachter, S., Sturch, L. K., Widhalm, A. M., Weiler, K. P., Pretorius, M. L., Wellhouse, J. W. \& Gibiansky, M. (2007), 'Cool Companions to White Dwarf Stars from the Two Micron All Sky Survey All Sky Data Release’, AJ 134, 26-42.

Hodapp, K. W., Jensen, J. B., Irwin, E. M., Yamada, H., Chung, R., Fletcher, K., Robertson, L., Hora, J. L., Simons, D. A., Mays, W., Nolan, R., Bec, M., Merrill, M. \& Fowler, A. M. (2003), 'The Gemini Near-Infrared Imager (NIRI)', PASP 115, 1388-1406.

Hoddeson, L. H. \& Baym, G. (1980), 'The Development of the Quantum Mechanical Electron Theory of Metals: 1900-28', Royal Society of London Proceedings Series A 371, 8-23.

Holberg, J. B. (2009), 'What fraction of white dwarfs are members of binary systems?', Journal of Physics Conference Series 172(1), 012022.

Holberg, J. B. \& Bergeron, P. (2006), 'Calibration of Synthetic Photometry Using DA White Dwarfs', $A J$ $132,1221-1233$. 
Holberg, J. B. \& Magargal, K. (2005), Finding the Cool Companions of the PG DA White Dwarfs, in D. Koester \& S. Moehler, eds, '14th European Workshop on White Dwarfs', Vol. 334 of Astronomical Society of the Pacific Conference Series, p. 419.

Iben, Jr., I. \& Renzini, A. (1983), 'Asymptotic giant branch evolution and beyond', A\&A 21, 271-342.

Ivanov, V. D., Rieke, M. J., Engelbracht, C. W., Alonso-Herrero, A., Rieke, G. H. \& Luhman, K. L. (2004), 'A Medium-Resolution Near-Infrared Spectral Library of Late-Type Stars. I.', ApJS 151, 387-397.

Jeans, J. H. (1924), 'Cosmogonic problems associated with a secular decrease of mass', MNRAS 85, 2.

Jones, H. R. A., Longmore, A. J., Jameson, R. F. \& Mountain, C. M. (1994), 'An infrared spectral sequence for M dwarfs', MNRAS 267, 413-423.

Jordan, S., Finley, D., Koester, D. \& Wolff, B. (1996), X-ray and EUV detection of metals in the atmospheres of hot DA white dwarfs., in H. U. Zimmermann, J. Trümper \& H. Yorke, eds, 'Roentgenstrahlung from the Universe', pp. 5-8.

Jura, M. (2003), 'A Tidally Disrupted Asteroid around the White Dwarf G29-38', ApJL 584, L91-L94.

Jura, M., Farihi, J. \& Zuckerman, B. (2009), 'Six White Dwarfs with Circumstellar Silicates', AJ 137, 3191-3197.

Jura, M., Farihi, J., Zuckerman, B. \& Becklin, E. E. (2007), 'Infrared Emission from the Dusty Disk Orbiting GD 362, an Externally Polluted White Dwarf', AJ 133, 1927-1933.

Kafka, S. \& Honeycutt, R. K. (2006), 'Spectroscopy of Active and Inactive M Dwarfs in Praesepe', $A J$ $132,1517-1526$.

Kalirai, J. S., Hansen, B. M. S., Kelson, D. D., Reitzel, D. B., Rich, R. M. \& Richer, H. B. (2008), 'The Initial-Final Mass Relation: Direct Constraints at the Low-Mass End', ApJ 676, 594-609.

Kawka, A., Vennes, S., Schmidt, G. D., Wickramasinghe, D. T. \& Koch, R. (2007), 'Spectropolarimetric Survey of Hydrogen-rich White Dwarf Stars', ApJ 654, 499-520.

Kilic, M., Allende Prieto, C., Brown, W. R. \& Koester, D. (2007b), 'The Lowest Mass White Dwarf', ApJ 660, 1451-1461.

Kilic, M., von Hippel, T., Leggett, S. K. \& Winget, D. E. (2005), 'Excess Infrared Radiation from the Massive DAZ White Dwarf GD 362: A Debris Disk?', ApJL 632, L115-L118.

Kilic, M., von Hippel, T., Leggett, S. K. \& Winget, D. E. (2006), 'Debris Disks around White Dwarfs: The DAZ Connection', ApJ 646, 474-479.

King, A. R., Frank, J., Kolb, U. \& Ritter, H. (1997), 'Mass Transfer Cycles in Close Binaries with Evolved Companions', ApJ 482, 919.

Kirkpatrick, J. D. (1998), The Definition of Spectral Type "L" and the 2MASS Search for Field Brown Dwarfs, in 'Bulletin of the American Astronomical Society', Vol. 30 of Bulletin of the American Astronomical Society, p. 1374.

Kirkpatrick, J. D. (2005), 'New Spectral Types L and T', ARA\&A 43, 195-245.

Kirkpatrick, J. D., Allard, F., Bida, T., Zuckerman, B., Becklin, E. E., Chabrier, G. \& Baraffe, I. (1999), 'An Improved Optical Spectrum and New Model FITS of the Likely Brown Dwarf GD 165B', ApJ 519, 834-843.

Kirkpatrick, J. D., Henry, T. J. \& Liebert, J. (1993), 'The unique spectrum of the brown dwarf candidate GD 165B and comparison to the spectra of other low-luminosity objects', ApJ 406, 701-707.

Kirkpatrick, J. D., Henry, T. J. \& McCarthy, Jr., D. W. (1991), 'A standard stellar spectral sequence in the red/near-infrared - Classes K5 to M9', APJS 77, 417-440.

Knutson, H. A., Charbonneau, D., Allen, L. E., Fortney, J. J., Agol, E., Cowan, N. B., Showman, A. P., Cooper, C. S. \& Megeath, S. T. (2007), 'A map of the day-night contrast of the extrasolar planet HD 189733b', Nature 447, 183-186. 
Koen, C. \& Maxted, P. F. L. (2006), 'Photometry of the magnetic white dwarf SDSS 121209.31+013627.7', MNRAS 371, 1675-1680.

Koester, D. (1991), A new look at old friends : 40 Eri B and GD 323, in G. Vauclair \& E. Sion, ed., 'NATO ASIC Proc. 336: White Dwarfs', p. 343.

Koester, D., Kepler, S. O., Kleinman, S. J. \& Nitta, A. (2009), 'SDSS white dwarf mass distribution at low effective temperatures', Journal of Physics Conference Series 172(1), 012006.

Koester, D. \& Reimers, D. (1981), 'Spectroscopic identification of white dwarfs in galactic clusters. I NGC 2287 and NGC 2422', A\&A 99, L8-L11.

Koester, D. \& Reimers, D. (1985), 'Spectroscopic identification of white dwarfs in galactic clusters. III IC 2391 and NGC 2451', A\&A 153, 260-264.

Koester, D. \& Reimers, D. (1993), 'Spectroscopic Identification of White Dwarfs in Galactic Clusters Part Six - Three New White Dwarfs in NGC3532', A\&A 275, 479.

Koester, D. \& Reimers, D. (1996), 'White dwarfs in open clusters. VIII. NGC 2516: a test for the massradius and initial-final mass relations.', $A \& A 313,810-814$.

Laughlin, G., Bodenheimer, P. \& Adams, F. C. (1997), 'The End of the Main Sequence', ApJ 482, 420.

Lépine, S. \& Shara, M. M. (2005), 'A Catalog of Northern Stars with Annual Proper Motions Larger than 0.15 ” (LSPM-NORTH Catalog)', AJ 129, 1483-1522.

Liebert, J., Bergeron, P., Eisenstein, D., Harris, H. C., Kleinman, S. J., Nitta, A. \& Krzesinski, J. (2004), 'A Helium White Dwarf of Extremely Low Mass', ApJL 606, L147-L149.

Liebert, J., Bergeron, P. \& Holberg, J. B. (2005), 'DA white dwarfs from the Palomar Green Survey (Liebert+, 2005)', VizieR Online Data Catalog 215, 60047.

Littlefair, S. P., Dhillon, V. S., Marsh, T. R., Gänsicke, B. T., Baraffe, I. \& Watson, C. A. (2007), 'SDSS J150722.30+523039.8: a cataclysmic variable formed directly from a detached white dwarf/brown dwarf binary?', MNRAS 381, 827-834.

Littlefair, S. P., Dhillon, V. S., Marsh, T. R., Gänsicke, B. T., Southworth, J. \& Watson, C. A. (2006), 'A Brown Dwarf Mass Donor in an Accreting Binary', Science 314, 1578.

Livio, M. \& Soker, N. (1983), 'Star-planet systems as progenitors of cataclysmic binaries Tidal effects', $A \& A \quad 125, \mathrm{~L} 12-\mathrm{L} 15$.

Livio, M. \& Soker, N. (1984), 'Star-planet systems as possible progenitors of cataclysmic binaries', $M N$ RAS 208, 763-781.

MacDonald, J. (1989), Low mass hydrogen envelopes and the DB gap, in G. Wegner, ed., 'IAU Colloq. 114: White Dwarfs', Vol. 328 of Lecture Notes in Physics, Berlin Springer Verlag, pp. 172-175.

Maraston, C. (1998), 'Evolutionary synthesis of stellar populations: a modular tool', MNRAS 300, 872892.

Maraston, C. (2005), 'Evolutionary population synthesis: models, analysis of the ingredients and application to high-z galaxies', MNRAS 362, 799-825.

Marcy, G. W. \& Butler, R. P. (2000), 'Planets Orbiting Other Suns', PASP 112, 137-140.

Marigo, P., Bressan, A. \& Chiosi, C. (1996), 'The TP-AGB phase: a new model.', A\&A 313, 545-564.

Maxted, P. F. L., Napiwotzki, R., Dobbie, P. D. \& Burleigh, M. R. (2006), 'Survival of a brown dwarf after engulfment by a red giant star', Nature 442, 543-545.

Maxted, P. F. L., O’Donoghue, D., Morales-Rueda, L., Napiwotzki, R. \& Smalley, B. (2007), 'The mass and radius of the M-dwarf in the short-period eclipsing binary RR Caeli', MNRAS 376, 919-928.

McCarthy, C. \& Zuckerman, B. (2004), 'The Brown Dwarf Desert at 75-1200 AU', AJ 127, 2871-2884. 
McCook, G. P. \& Sion, E. M. (1999), 'A Catalog of Spectroscopically Identified White Dwarfs', ApJS $121,1-130$.

McLean, I. S. (2003), Near-infrared spectroscopic survey of brown dwarfs using NIRSPEC on the Keck II Telescope, in P. Guhathakurta, ed., 'Discoveries and Research Prospects from 6- to 10-Meter-Class Telescopes II. Edited by Guhathakurta, Puragra. Proceedings of the SPIE, Volume 4834, pp. 111-118 (2003).', Vol. 4834 of Presented at the Society of Photo-Optical Instrumentation Engineers (SPIE) Conference, pp. 111-118.

Metcalfe, T. S., Montgomery, M. H. \& Kanaan, A. (2004), 'Testing White Dwarf Crystallization Theory with Asteroseismology of the Massive Pulsating DA Star BPM 37093', ApJL 605, L133-L136.

Meyer, M. R., Edwards, S., Hinkle, K. H. \& Strom, S. E. (1998), 'Near-Infrared Classification Spectroscopy: H-Band Spectra of Fundamental MK Standards', ApJ 508, 397-409.

Morgan, W. W., Keenan, P. C. \& Kellman, E. (1943), An atlas of stellar spectra, with an outline of spectral classification.

Mullally, F., Kilic, M., Reach, W. T., Kuchner, M. J., von Hippel, T., Burrows, A. \& Winget, D. E. (2006), 'A Spitzer White Dwarf Infrared Survey', ArXiv Astrophysics e-prints .

Mullally, F., Kilic, M., Reach, W. T., Kuchner, M. J., von Hippel, T., Burrows, A. \& Winget, D. E. (2007), 'A Spitzer White Dwarf Infrared Survey', ApJS 171, 206-218.

Nelemans, G. \& Tauris, T. M. (1998), 'Formation of undermassive single white dwarfs and the influence of planets on late stellar evolution', $A \& A$ 335, L85-L88.

Patten, B. M., Stauffer, J. R., Burrows, A., Marengo, M., Hora, J. L., Luhman, K. L., Sonnett, S. M., Henry, T. J., Raghavan, D., Megeath, S. T., Liebert, J. \& Fazio, G. G. (2006), 'Spitzer IRAC Photometry of $\mathrm{M}, \mathrm{L}$, and T Dwarfs', ApJ 651, 502-516.

Pinfield, D. J., Jones, H. R. A., Lucas, P. W., Kendall, T. R., Folkes, S. L., Day-Jones, A. C., Chappelle, R. J. \& Steele, I. A. (2006), 'Finding benchmark brown dwarfs to probe the substellar initial mass function as a function of time', MNRAS 368, 1281-1295.

Probst, R. G. (1983), 'An infrared search for very low mass stars - JHK photometry and results for composite systems', ApJS 53, 335-349.

Renzini, A. \& Voli, M. (1981), 'Advanced evolutionary stages of intermediate-mass stars. I - Evolution of surface compositions', $A \& A$ 94, 175-193.

Romanishin, W. \& Angel, J. R. P. (1980), 'Determination of the upper mass limit for stars producing white-dwarf remnants', ApJ 235, 992-998.

Rowell, N. R., Hambly, N. C. \& Bergeron, P. (2009), 'The luminosity function for white dwarfs in the SuperCOSMOS Sky Survey', Journal of Physics Conference Series 172(1), 012001.

Schmidt, G. D., Szkody, P., Silvestri, N. M., Cushing, M. C., Liebert, J. \& Smith, P. S. (2005), 'Discovery of a Magnetic White Dwarf/Probable Brown Dwarf Short-Period Binary', ApJL 630, L173-L176.

Schönberg, M. \& Chandrasekhar, S. (1942), 'On the Evolution of the Main-Sequence Stars.', ApJ 96, 161.

Schreiber, M. R. \& Gänsicke, B. T. (2003), 'The age, life expectancy, and space density of Post Common Envelope Binaries', AAP 406, 305-321.

Skrutskie, M. F., Beichman, C., Capps, R., Carpenter, J., Chester, T., Cutri, R., Elias, J., Elston, R., Huchra, J., Liebert, J., Lonsdale, C., Monet, D., Price, S., Schneider, S., Seitzer, P., Stiening, R., Strom, S. \& Weinberg, M. (1995), The Two Micron All Sky Survey (2MASS), in 'Bulletin of the American Astronomical Society', Vol. 27 of Bulletin of the American Astronomical Society, p. 1392.

Skrutskie, M. F., Cutri, R. M., Stiening, R., Weinberg, M. D., Schneider, S., Carpenter, J. M., Beichman, C., Capps, R., Chester, T., Elias, J., Huchra, J., Liebert, J., Lonsdale, C., Monet, D. G., Price, S., Seitzer, P., Jarrett, T., Kirkpatrick, J. D., Gizis, J. E., Howard, E., Evans, T., Fowler, J., Fullmer, L., Hurt, R., Light, R., Kopan, E. L., Marsh, K. A., McCallon, H. L., Tam, R., Van Dyk, S. \& Wheelock, S. (2006), 'The Two Micron All Sky Survey (2MASS)', AJ 131, 1163-1183. 
Steele, P. R., Burleigh, M. R., Farihi, J., Gänsicke, B. T., Jameson, R. F., Dobbie, P. D. \& Barstow, M. A. (2009), 'PHL 5038: a spatially resolved white dwarf + brown dwarf binary', A\&A 500, 1207-1210.

Stephens, D. C., Marley, M. S., Noll, K. S. \& Chanover, N. (2001), 'L-Band Photometry of L and T Dwarfs', ApJL 556, L97-L101.

Su, K. Y. L., Chu, Y.-H., Rieke, G. H., Huggins, P. J., Gruendl, R., Napiwotzki, R., Rauch, T., Latter, W. B. \& Volk, K. (2007), 'A Debris Disk around the Central Star of the Helix Nebula?', ApJL 657, L41L45.

Tappert, C., Gänsicke, B. T., Schmidtobreick, L., Mennickent, R. E. \& Navarrete, F. P. (2007), 'K-band spectroscopy of pre-cataclysmic variables', $A \& A \quad 475,575-583$.

Tremblay, P.-E. \& Bergeron, P. (2007), Near-Infrared Photometric Analyzes of White Dwarf Stars, in R. Napiwotzki \& M. R. Burleigh, eds, '15th European Workshop on White Dwarfs', Vol. 372 of Astronomical Society of the Pacific Conference Series, p. 283.

Tremblay, P.-E. \& Bergeron, P. (2007b), 'Infrared Photometric Analysis of White Dwarfs from the Two Micron All Sky Survey and the Spitzer Space Telescope', ApJ 657, 1013.

Vacca, W. D., Cushing, M. C. \& Rayner, J. T. (2003), 'A Method of Correcting Near-Infrared Spectra for Telluric Absorption', PASP 115, 389-409.

Vanlandingham, K. M., Schmidt, G. D., Eisenstein, D. J., Harris, H. C., Anderson, S. F., Hall, P. B., Liebert, J., Schneider, D. P., Silvestri, N. M., Stinson, G. S. \& Wolfe, M. A. (2005), 'Magnetic White Dwarfs from the SDSS. II. The Second and Third Data Releases', AJ 130, 734-741.

Vennes, S., Smith, R. J., Boyle, B. J., Croom, S. M., Kawka, A., Shanks, T., Miller, L. \& Loaring, N. (2002), 'DA white dwarfs in 2dF QSO Redshift Survey (Vennes+, 2002)', VizieR Online Data Catalog 733, 50673.

Villaver, E. \& Livio, M. (2007), 'Can Planets Survive Stellar Evolution?', ApJ 661, 1192-1201.

von Hippel, T., Kuchner, M. J., Kilic, M., Mullally, F. \& Reach, W. T. (2007), 'The New Class of Dusty DAZ White Dwarfs', ApJ 662, 544-551.

Vrba, F. J., Henden, A. A., Luginbuhl, C. B., Guetter, H. H., Munn, J. A., Canzian, B., Burgasser, A. J., Kirkpatrick, J. D., Fan, X., Geballe, T. R., Golimowski, D. A., Knapp, G. R., Leggett, S. K., Schneider, D. P. \& Brinkmann, J. (2004), 'Preliminary Parallaxes of 40 L and T Dwarfs from the US Naval Observatory Infrared Astrometry Program’, AJ 127, 2948-2968.

Wachter, S., Hoard, D. W., Hansen, K. H., Wilcox, R. E., Taylor, H. M. \& Finkelstein, S. L. (2003), 'Cool Companions to White Dwarfs from the Two Micron All-Sky Survey Second Incremental Data Release', ApJ 586, 1356-1363.

Wallace, L. \& Hinkle, K. (1997), 'Medium-Resolution Spectra of Normal Stars in the K Band', ApJS 111,445 .

Wallace, L., Meyer, M. R., Hinkle, K. \& Edwards, S. (2000), 'Near-Infrared Classification Spectroscopy: J-Band Spectra of Fundamental MK Standards', ApJ 535, 325-337.

Weidemann, V. (1977), 'Mass loss towards the white dwarf stage', A\&A 59, 411-418.

Weidemann, V. (1987), 'The initial-final mass relation - Galactic disk and Magellanic Clouds', $A \& A$ $188,74-84$.

Weidemann, V. (2000), 'Revision of the initial-to-final mass relation', $A \& A$ 363, 647-656.

Weidemann, V. \& Koester, D. (1983), 'The upper mass limit for white dwarf progenitors and the initialfinal mass relation for low and intermediate mass stars', $A \& A$ 121, 77-84.

Weidemann, V. \& Koester, D. (1984), 'Mass distribution of DA white dwarfs and atmospheric parameters of ZZ Ceti stars', A\&A 132, 195-202.

Wellhouse, J. W., Hoard, D. W., Howell, S. B., Wachter, S. \& Esin, A. A. (2005), 'Magnetic White Dwarfs in the Two Micron All Sky Survey: A Search for Candidate Binary Systems', PASP 117, 1378-1385. 
Williams, K. A., Bolte, M. \& Koester, D. (2004), 'An Empirical Initial-Final Mass Relation from Hot, Massive White Dwarfs in NGC 2168 (M35)', ApJL 615, L49-L52.

Winget, D. E. (1995), 'The Status of White Dwarf Asteroseismology and a Glimpse of the Road Ahead', Baltic Astronomy 4, 129-136.

Wolff, B., Koester, D., Dreizler, S. \& Haas, S. (1998), 'Photospheric metals in hot DA white dwarfs', $A \& A$ $329,1045-1058$.

Wood, J. H. \& Horne, K. (1990), 'White dwarf radii and boundary-layer constraints in three dwarf novae', MNRAS 242, 606-615.

Wood, M. A. (1992), 'Constraints on the age and evolution of the Galaxy from the white dwarf luminosity function', ApJ 386, 539-561.

York, D. G., Adelman, J., Anderson, Jr., J. E., Anderson, S. F., Annis, J., Bahcall, N. A., Bakken, J. A., Barkhouser, R., Bastian, S., Berman, E., Boroski, W. N., Bracker, S., Briegel, C., Briggs, J. W., Brinkmann, J., Brunner, R., Burles, S., Carey, L., Carr, M. A., Castander, F. J., Chen, B., Colestock, P. L., Connolly, A. J., Crocker, J. H., Csabai, I., Czarapata, P. C., Davis, J. E., Doi, M., Dombeck, T., Eisenstein, D., Ellman, N., Elms, B. R., Evans, M. L., Fan, X., Federwitz, G. R., Fiscelli, L., Friedman, S., Frieman, J. A., Fukugita, M., Gillespie, B., Gunn, J. E., Gurbani, V. K., de Haas, E., Haldeman, M., Harris, F. H., Hayes, J., Heckman, T. M., Hennessy, G. S., Hindsley, R. B., Holm, S., Holmgren, D. J., Huang, C.-h., Hull, C., Husby, D., Ichikawa, S.-I., Ichikawa, T., Ivezić, Ž., Kent, S., Kim, R. S. J., Kinney, E., Klaene, M., Kleinman, A. N., Kleinman, S., Knapp, G. R., Korienek, J., Kron, R. G., Kunszt, P. Z., Lamb, D. Q., Lee, B., Leger, R. F., Limmongkol, S., Lindenmeyer, C., Long, D. C., Loomis, C., Loveday, J., Lucinio, R., Lupton, R. H., MacKinnon, B., Mannery, E. J., Mantsch, P. M., Margon, B., McGehee, P., McKay, T. A., Meiksin, A., Merelli, A., Monet, D. G., Munn, J. A., Narayanan, V. K., Nash, T., Neilsen, E., Neswold, R., Newberg, H. J., Nichol, R. C., Nicinski, T., Nonino, M., Okada, N., Okamura, S., Ostriker, J. P., Owen, R., Pauls, A. G., Peoples, J., Peterson, R. L., Petravick, D., Pier, J. R., Pope, A., Pordes, R., Prosapio, A., Rechenmacher, R., Quinn, T. R., Richards, G. T., Richmond, M. W., Rivetta, C. H., Rockosi, C. M., Ruthmansdorfer, K., Sandford, D., Schlegel, D. J., Schneider, D. P., Sekiguchi, M., Sergey, G., Shimasaku, K., Siegmund, W. A., Smee, S., Smith, J. A., Snedden, S., Stone, R., Stoughton, C., Strauss, M. A., Stubbs, C., SubbaRao, M., Szalay, A. S., Szapudi, I., Szokoly, G. P., Thakar, A. R., Tremonti, C., Tucker, D. L., Uomoto, A., Vanden Berk, D., Vogeley, M. S., Waddell, P., Wang, S.-i., Watanabe, M., Weinberg, D. H., Yanny, B. \& Yasuda, N. (2000), 'The Sloan Digital Sky Survey: Technical Summary', AJ 120, 1579-1587.

Zuckerman, B. \& Becklin, E. E. (1987a), 'A search for brown dwarfs and late M dwarfs in the Hyades and the Pleiades', ApJL 319, L99-L102.

Zuckerman, B. \& Becklin, E. E. (1987b), 'Excess infrared radiation from a white dwarf - an orbiting brown dwarf?', Nature 330, 138-140.

Zuckerman, B. \& Becklin, E. E. (1992), 'Companions to white dwarfs - Very low-mass stars and the brown dwarf candidate GD 165B', ApJ 386, 260-264.

Zuckerman, B., Koester, D., Reid, I. N. \& Hünsch, M. (2003), 'Metal Lines in DA White Dwarfs', ApJ 596, 477-495. 